\title{
Identitätskonstruktion und Verfasserreferenz in deutschen und US-amerikanischen wissenschaftlichen Artikeln
}

\author{
Dissertation \\ zur Erlangung des Doktorgrades (Dr. phil.) im Fach \\ Deutsche Philologie (Deutsche Sprachwissenschaft, \\ Interkulturelle Germanistik), \\ der

\section{Philosophischen Fakultät \\ der Georg-August Universität Göttingen} \\ vorgelegt von

\section{Julia Schmidt M.A.,} \\ geboren am 17. Januar 1978 in Bad Gandersheim.
}

Erstgutachterin

Prof. Dr. Hiltraud Casper-Hehne

Seminar für Deutsche Philologie (Göttingen)

Zweitgutachter

Prof. Dr. Albert Busch

Seminar für Deutsche Philologie (Göttingen) 


\section{Danksagung}

Mein herzlicher Dank gilt zunächst meiner Erstbetreuerin Prof. Dr. Hiltraud CasperHehne und meinem Zweitbetreuer Prof. Dr. Albert Busch, die diese Arbeit nicht nur inhaltlich, sondern auch in vielen anderen Bereichen unterstützt haben.

Durch gewinnbringende Diskussionen und hilfreiches Feedback haben die Mitglieder des Kolloquiums Interkulturelle Germanistik meine Perspektive und damit diese Arbeit erweitert und mit geprägt.

Weiterhin danke ich Dr. Melanie Brinkschulte für ihre zielgerichtete schreibdidaktische Unterstützung sowie dem Team der Graduiertenschule für Geisteswissenschaften Göttingen (GSGG), insbesondere Dr. Nele Hoffmann, für strukturellen, aber vor allem auch kollegialen Rückhalt.

Für umfassende Rückmeldungen zu Verständlichkeit und Kohärenz sowie sorgfältiges und genaues Korrekturlesen danke ich Dr. Alina Rull und Florian Schnürer.

Ein ganz besonderer Dank geht an Dr. Annegret Middeke, Dr. Yassir El Jamouhi, Dr. Nadine Stahlberg und Angelika Thielsch, die durch ihr fachliches Feedback, unterschiedlichste Motivationshilfen und insbesondere ihre Freundschaft maßgeblich zum Gelingen dieser Arbeit beigetragen haben. 


\section{Inhaltsverzeichnis}

\begin{tabular}{lll}
\hline & Einleitung & 6
\end{tabular}

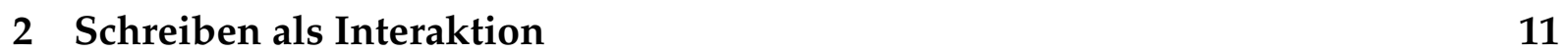

2.1 Schreiben als Interaktion . . . . . . . . . . . . . . . . . . . . . 12

2.2 Wissenschaftliches Schreiben und Autorenidentität . . . . . . . . . . 17

2.2 .1 Identität . . . . . . . . . . . . . . . . . . . . 19

2.2.1.1 Identität und Sprache . . . . . . . . . . . . . . . . 21

2.2.1.2 Identität und Selbstdarstellung im wissenschaftlichen

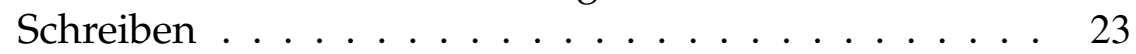

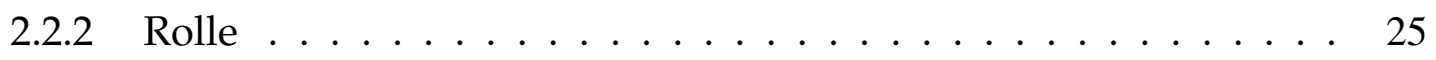

2.2 .3 Wissenschaftskultur und Diskursgemeinschaft . . . . . . . . . 30

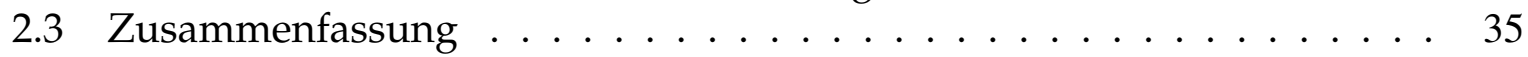

$\begin{array}{lll}3 & \text { Selbstdarstellung in wissenschaftlichen Artikeln } & 37\end{array}$

3.1 Die Textsorte "Wissenschaftlicher Artikel ${ }^{\prime \prime}$. . . . . . . . . . . . . . . . . 37

3.2 Theoretische Ansätze $\ldots \ldots \ldots \ldots \ldots$. . . . . . . . . . . . 40

3.2 .1 Voice . . . . . . . . . . . . . . . . . . . . . . . 40

3.2 .2 Stance and engagement . . . . . . . . . . . . . . . . . . 42

3.3 Sprachliche Mittel der Selbstdarstellung . . . . . . . . . . . . . . . . . . . 44

3.3 .1 Personalpronomen . . . . . . . . . . . . . . . . 46

3.3.1.1 1. Person Singular . . . . . . . . . . . . . . . . . . . . . 47

3.3.1.2 1. Person Plural $\ldots \ldots \ldots$. . . . . . . . . . . . . . . . 59

3.3.1.3 Unpersönliches MAN . . . . . . . . . . . . . . . . . . . . 67

3.3 .2 Autorenrollen . . . . . . . . . . . . . . . . . . 70

3.3.2.1 Autorenidentitäten nach Tang und John (1999) . . . . . 71

3.3.2.2 Rhetorische Funktionen - die KIAP-Studie . . . . . . . . 75

3.3.2.3 Autorenrollen nach Steinhoff . . . . . . . . . . . . 76

3.3.2.4 Diskursfunktionen nach Hyland . . . . . . . . . . . . . . 78

3.3.2.5 Autorenrollen und wissenschaftliche Identität. . . . . . 80

3.4 Zusammenfassung . . . . . . . . . . . . . . 83

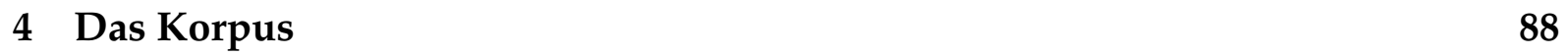

$\begin{array}{lll}5 & \text { Auswertung } & 98\end{array}$

5.1 Beschreibung der Rollen . . . . . . . . . . . . . . . . . . . . . . 100

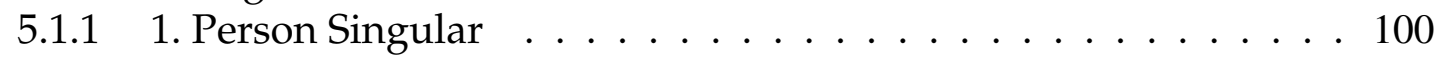

5.1 .1 .1 Verfasser . . . . . . . . . . . . . . . 100

5.1.1.1.1 Verbale und graphische Darstellungen . . . . . 101

5.1.1.1.2 Darstellen des Ziels bzw. des Fokus des Textes 101

5.1.1.1.3 Theoretischer Hintergrund . . . . . . . . . 102 
5.1.1.1.4 Terminologieklärung . . . . . . . . . . . 103

5.1.1.1.5 Textstrukturierung . . . . . . . . . . . 103

5.1.1.1.6 Sonstige Metakommunikation . . . . . . . . . . 104

5.1 .1 .2 Forscher . . . . . . . . . . . . . . . . . 104

5.1.1.2.1 Fokussieren der Forschungsfrage . . . . . . . . 105

5.1.1.2.2 Auswählen von Hypothesen, Ausgangsannahmen und theoretischen Grundpositionen . . . 106

5.1.1.2.3 Daten erheben . . . . . . . . . . . . . 107

5.1.1.2.4 Daten aufbereiten und analysieren . . . . . . . . 108

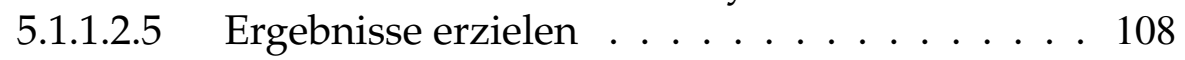

5.1.1.2.6 Argumentieren für den eigenen Standpunkt. . 109

5.1.1.2.7 Aufstellen neuer Claims und neuer Ideen . . . 109

5.1.1.2.8 Danksagungen . . . . . . . . . . . . . . 110

5.1 .1 .3 Andere . . . . . . . . . . . . . . 112

5.1 .2 1. Person Plural . . . . . . . . . . . . . . . . . . . . . . . . . 113

5.1.2.1 Exklusives WIR . . . . . . . . . . . . . . . . . . . . . 114

5.1.2.1.1 WIR als Autor . . . . . . . . . . . . . . . . 114

5.1.2.1.2 Autor und andere . . . . . . . . . . . . . . 116

5.1.2.2 Pseudo-inklusives WIR . . . . . . . . . . . . . . 116

5.1 .2 .3 Inklusives WIR . . . . . . . . . . . . . . . . . 117

5.1.2.3.1 Autor und Leser: Teamwork-WIR . . . . . . . . 117

5.1.2.3.2 Autor und Fachcommunity: Fachkreis-WIR . . 118

5.1.2.3.3 Autor und die weitere Gemeinschaft: Gemeinschafts-WIR . . . . . . . . . . . 119

5.1.2.4 Rollen in der ersten Person Plural . . . . . . . . . . . . . 120

5.1 .2 .4 .1 Verfasser . . . . . . . . . . . . 121

5.1 .2 .4 .2 Forscher . . . . . . . . . . . . . 122

5.1 .3 Unpersönliches MAN . . . . . . . . . . . . . . . . . . . . . 127

5.1.3.1 Exklusives MAN . . . . . . . . . . . . . . . . . . . . . 127

5.1 .3 .1 .1 Nur Autor . . . . . . . . . . . . . . . . . . . 127

5.1 .3 .1 .2 Nicht Autor . . . . . . . . . . . . . . . . 127

5.1 .3 .2 Inklusives MAN . . . . . . . . . . . . . . . . . . . . . 128

5.1 .3 .2 .1 Teamwork-MAN . . . . . . . . . . . . . 128

$5.1 .3 .2 .2 \quad$ Fachkreis-MAN . . . . . . . . . . . . . . . . 129

5.1.3.2.3 Gemeinschafts-MAN . . . . . . . . . . . . . . . 129

5.1.3.3 Rollen mit dem unpersönlichen MAN . . . . . . . . . . 130

5.1 .4 Zusammenfassung . . . . . . . . . . . . . . . . . 134

5.2 Die Pronomen im interlingualen Vergleich . . . . . . . . . . . . . . . 135

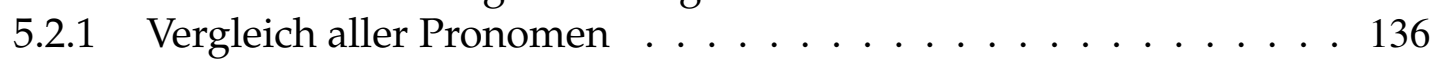

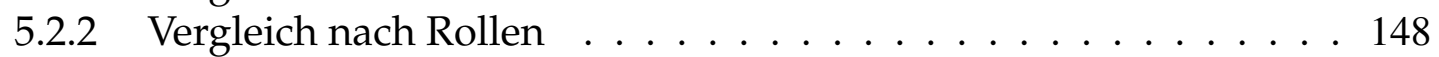

5.2.2.1 Vergleich 1. Person Singular . . . . . . . . . . . . 148

5.2.2.1.1 Verfasser . . . . . . . . . . . . . . 149

5.2 .2 .1 .2 Forscher . . . . . . . . . . . . . . 158

5.2 .2 .1 .3 Andere . . . . . . . . . . . . . . . . 172

5.2.2.2 Vergleich 1. Person Plural. . . . . . . . . . . . . . . . . 172

5.2.2.2.1 $\quad$ Verfasser . . . . . . . . . . . . . . 179

5.2 .2 .2 .2 Forscher . . . . . . . . . . . . . . 181

5.2 .2 .2 .3 Andere . . . . . . . . . . . . . . . . . . . . . . . . . . 189

5.2 .3 Zusammenfassung . . . . . . . . . . . . . . . . 189 
5.3 Fallstudie Vaughan . . . . . . . . . . . . . . . . . . . . . . . . 195

5.4 Exkurs: Impression Management . . . . . . . . . . . . . . . . . . . . 202

5.4 .1 Positives Impression Management . . . . . . . . . . . . . . . . . . 207

5.4 .2 Negatives Impression Management . . . . . . . . . . . . . . . 211

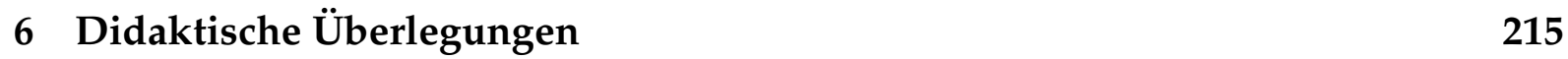

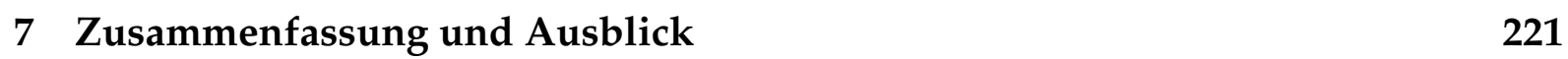

\begin{tabular}{lrr}
\hline A Korpustexte & 228
\end{tabular}

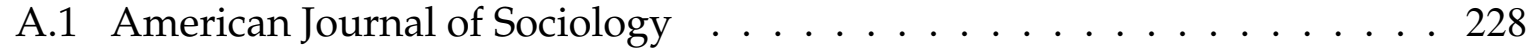

A.2 Kölner Zeitschrift für Soziologie und Sozialpsychologie . . . . . . . . . . 230 


\section{Kapitel 1}

\section{Einleitung}

„Darf ich in meiner Hausarbeit eigentlich ich schreiben?“ Diese Frage wird immer wieder von verunsicherten Studierenden in verschiedensten Veranstaltungen gestellt und auch von Lehrenden immer wieder diskutiert. Dieses Thema ist nicht neu und es gibt schon mehrere Untersuchungen und Überlegungen zum ICH-Gebrauch in wissenschaftlichen und akademischen Texten aus unterschiedlichen theoretischen Richtungen, im deutschsprachigen Raum vornehmlich der Fachtextanalyse und der Funktionalen Pragmatik, international spielen hier beispielsweise die Systemisch-Funktionale Linguistik oder die Genre-Analyse eine wichtige Rolle. Doch die Fragen der Studierenden sind geblieben und eine befriedigende Antwort darauf ist nur den wenigsten Lehrenden bekannt.

Dies kann unter anderem daran liegen, dass, zumindest im deutschsprachigen Bereich, dieses Problem vorrangig aus linguistischer Perspektive angegangen wurde, eine soziolinguistische Herangehensweise sich jedoch noch nicht durchsetzen konnte. Dabei ist die Frage nach dem ICH-Gebrauch - in jedem sprachlichen Kontext - sicherlich in erster Linie eine soziolinguistische, wenn nicht sogar eine soziologische oder sozialpsychologische Frage. Das ICH definiert in direkter Weise den Sprecher ${ }^{1}$ bzw. den Autor eines Textes und gibt Aufschluss darüber, welche Identität(en) er sich in der sprachlichen Interaktion zuschreibt, welchen Gruppen er sich zuordnet (und welchen dadurch auch nicht) und wie er generell von seinem Gegenüber wahrgenommen werden will. Dies ist eine Eigenschaft, die jeder sprachlichen Äußerung grundsätzlich zu Eigen ist, und es fällt schwer, sich einen natürlichen Kontext vorzustellen, in dem die (bewusste oder unbewusste) Konstruktion einer Identität und die damit einhergehende Positionierung des Sprechers bzw. Schreibers keine Rolle für die Kommunikation und die Interaktion spielt.

Als Leser eines wissenschaftlichen Textes beispielsweise macht man sich (meist unbewusst) immer ein Bild desjenigen, der diesen Text verfasst hat, und gelegentlich stellt

\footnotetext{
${ }^{1}$ In dieser Arbeit wird aus Gründen der Lesbarkeit das generische Maskulinum verwendet, dabei werden aber immer auch Autorinnen, Leserinnen, Wissenschaftlerinnen usw. mitgedacht. Weibliche Formen werden dann verwendet, wenn ausschließlich weibliche Personen gemeint werden.
} 
man dann fest, dass dieses Bild, das man sich beim Lesen konstruiert hat, nicht oder nur zum Teil der Realität entspricht. Beispielsweise "klingt“ ein Text eindeutig so, als sei er von einem jungen Wissenschaftler verfasst, möglicherweise sogar einem Studenten, man freut sich, dass der Text dieses Nachwuchswissenschaftlers veröffentlicht wurde und stellt beim Blick in die Autorenbiographien erstaunt fest, dass der Verfasser den Lehrstuhl für $X$ an der Universität $Y$ innehat. Und auch die entsprechende gegenteilige Diskrepanz zwischen Wahrnehmung und Realität kommt gelegentlich vor ${ }^{2}$

Die gelungene Darstellung seiner Selbst als kompetent, glaubwürdig und erfolgreich spielt folglich in der wissenschaftlichen Kommunikation eine mindestens gleichbedeutende Rolle, wie die Produktion und Verbreitung neuer wissenschaftlicher Erkenntnisse. Hier unterscheidet sich der Kommunikationsbereich „Wissenschaft" nicht wesentlich von anderen Feldern wie „Wirtschaft" , „Medien“ usw ${ }^{3}$ In der Wissenschaft spielt das schriftliche Medium, v. a. der in einer Fachzeitschrift veröffentlichte Artikel, jedoch eine besondere Rolle. Nichtsdestoweniger ist auch und gerade in schriftlichen wissenschaftlichen Texten die erfolgreiche Konstruktion einer angemessenen Identität grundlegend für eine erfolgreiche Kommunikation.

Erschwert wird dies dadurch, dass wissenschaftliches Schreiben inzwischen immer in einem heterogenen kulturellen Kontext stattfindet, wodurch es intrinsisch eine interkulturelle Kommunikation bzw. Interaktion ist. Der kulturelle Kontext wird hier zwar nicht nur, aber auch in Zusammenhang mit sprachlichen Unterschieden gesehen. Konkret ist beispielsweise das Englische auch für deutsche Wissenschaftler nicht mehr aus ihrem täglichen Berufsleben wegzudenken und das Veröffentlichen von Artikeln in englischsprachigen Fachzeitschriften gehört in den meisten Disziplinen zum wissenschaftlichen Alltagsgeschäft.

Aber auch innerhalb der eigenen Sprachgemeinschaft kann man interkulturelle Unterschiede im wissenschaftlichen Schreiben ausmachen, nicht nur über disziplinäre Grenzen zwischen den Geistes-, Sozial- und Naturwissenschaften hinweg. Auch innerhalb dieser durchaus häufig arbiträr verlaufenden Grenzen gibt es Unterschiede zwischen einzelnen Fächern (vgl. z. B. Hyland 2001b, 2002a; Fløttum u. a. 2006; Steinhoff 2007: Sanderson|2008a).

Aus diesem Grund soll in dieser Untersuchung, anders als in vielen anderen Studien mit ähnlichen Fragestellungen (s. Kap. 3), der Fokus auf einer einzelnen Fachdisziplin, nämlich der Soziologie, liegen. Dafür wird ein interlingualer Vergleich zwischen Artikeln gezogen, die in einer deutschen bzw. einer US-amerikanischen Zeitschrift veröffentlicht wurden, um die wesentlichen Unterschiede zwischen diesen beiden Wissenschaftskulturen (der deutschen und der US-amerikanischen Soziologie, repräsen-

\footnotetext{
${ }^{2}$ Vgl. dazu auch Tardy und Matsuda (2009: 32), die feststellen, , [...] that readers often form impressions of or speculate about unknown authors' identities in the manuscript review task".

${ }^{3}$ Zum Begriff des wissenschaftlichen Feldes s. z. B Bourdieu (1998)
} 
tiert durch ihre beiden führenden Fachzeitschriften) in Bezug auf die Selbstdarstellung und die Konstruktion von Identität im wissenschaftlichen Schreiben herauszuarbeiten.

Dies ist besonders interessant, da im deutschsprachigen Kontext im Rahmen dieser Fragestellung bisher nur vergleichsweise wenig zu sozialwissenschaftlichen Texten geforscht wurde. In den für die vorliegende Arbeit relevanten Untersuchungen (z. B. Kresta 1995; Steinhoff 2007; Sanderson 2008a) haben meist geisteswissenschaftliche Texte im Vordergrund gestanden. Zwar ist der Stil sozialwissenschaftlicher Autoren im Allgemeinen sicherlich eher vergleichbar mit dem geisteswissenschaftlicher Autoren, v. a. in Abgrenzung zu dem naturwissenschaftlicher Autoren. Es ist aber trotzdem anzunehmen, dass sich auch zwischen geisteswissenschaftlichen und sozialwissenschaftlichen Texten bedeutsame Unterschiede zeigen, da sich zumindest teilweise die epistemologischen Ausgangspunkte sowie nicht zuletzt die wissenschaftlichen Methoden, die in den beiden Disziplinen angewandt werden, deutlich unterscheiden.

Um der Frage nach der Konstruktion einer wissenschaftlichen Identität nachzugehen, wurde ein Korpus aus 50 soziologischen Texten (25 auf Deutsch, 25 auf Englisch) zusammengestellt. In diesem Korpus wurden die Pronomen der ersten Person im Singular und Plural sowie die unpersönlichen Pronomen MAN und ONE in Subjektposition lokalisiert, um ihren Gebrauch in den beiden Teilkorpora zu untersuchen und zu vergleichen. Dabei standen die Handlungen, für die diese Pronomen als Agens fungieren, im Vordergrund $4^{4}$ Anhand dieser Handlungen, die durch das Verb angezeigt werden, wurden die unterschiedlichen Gebrauchsweisen in Rollen kategorisiert, die die Identität "Wissenschaftlicher Autor" in den beiden betrachteten Wissenschaftskulturen charakterisieren.

Das zweite Kapitel stellt die grundlegenden Theorien und Ansätze im Bereich der Identitätsforschung vor, auf denen diese Arbeit basiert. Dafür wird das wissenschaftliche Schreiben, insbesondere das Verfassen wissenschaftlicher Aufsätze, als soziale Interaktion beschrieben. Wissenschaftliche Autoren müssen die sprachlichen und fachkulturellen Anforderungen ihrer Disziplin erfüllen, und damit ihre Artikel in einschlägigen Fachzeitschriften erscheinen können, müssen sie sich selbst als glaubwürdige und kompetente Wissenschaftler in ihren Texten präsentieren und eine entsprechende Identität als Wissenschaftlicher Autor konstruieren. Dies ist die Grundvoraussetzung dafür, dass sie einerseits die Reviewer und andererseits das breite Fachpublikum von ihren Forschungsergebnissen überzeugen können. Die zugrundeliegende Annahme ist hier, dass Identitäten durch soziale Rollen realisiert werden und dass diese Rollen sich wiederum anhand der Handlungen, die der Autor sich in seinem Text über den Gebrauch der ersten Person selbst zuschreibt, abgelesen werden können. Als Basis für diese Position werden in Kapitel 2 die Konzepte "Identität“ und „Rolle“ dargestellt

\footnotetext{
${ }^{4} \mathrm{Um}$ eine automatische Suche zu ermöglichen, wurde der Analysefokus auf diejenigen Pronomen beschränkt, die gleichzeitig das syntaktische Subjekt der untersuchten Sätze, deren Prädikat die Autorenrolle bzw. -handlung beschreibt, stellen.
} 
und wichtige Ergebnisse zur Identitätskonstruktion im wissenschaftlichen Schreiben referiert. Da die Auswahl der Rollen und v. a. der Handlungen, die diese Rollen symbolisieren, auch kulturspezifischen Parametern unterliegt, wird am Schluss des zweiten Kapitels außerdem das in dieser Arbeit angewandte Konzept von „Wissenschaftskultur" vorgestellt.

Im dritten Kapitel werden dann wesentliche Merkmale wissenschaftlicher Artikel beleuchtet, um daraufhin wichtige theoretische Ansätze und bisherige Ergebnisse zum Gebrauch von Personalpronomen der ersten Person sowie des unpersönlichen MAN vorzustellen. Es werden einschlägige Studien zum Gebrauch dieser Pronomen im deutschen und englischen wissenschaftlichen und akademischen Schreiben diskutiert und insbesondere diejenigen Untersuchungen berücksichtigt, die einen interaktionsorientierten Ansatz vertreten. Daran anschließend wird das Konzept der Autorenrollen ebenfalls an bisher erzielten empirischen Ergebnissen dargestellt. Darauf basierend wird ein Kategoriensystem für die im deutschen und englischsprachigen wissenschaftlichen Schreiben relevanten Autorenrollen erarbeitet, das als Grundlage für die Auswertung der Korpustexte dient. Abschließend wird die theoretische Perspektive zusammengefasst.

Im empirischen Teil dieser Arbeit werden die Ergebnisse aus der Untersuchung des hier verwendeten Korpus dargestellt. Das Korpus selbst wird in Kapitel 4 vorgestellt. Es umfasst insgesamt 50 veröffentlichte Texte aus der Kölner Zeitschrift für Soziologie und Sozialpsychologie und dem American Journal of Sociology. Die Texte wurden in der Zeit von 2005 bis 2010 veröffentlicht und aus der Gesamtmenge aller in den beiden Zeitschriften veröffentlichten Artikel, die von einem Einzelautor verfasst wurden, zufällig ausgewählt.

Die Auswertung dieser Texte wird in Kapitel 5 vorgestellt. Zunächst werden die Handlungskategorien der ersten Person Singular und Plural sowie ergänzend auch des unpersönlichen MAN, die sich aus der Forschungsliteratur (Kap. 3) sowie der Analyse des Korpus für die Fragestellung dieser Arbeit als relevant herausgestellt haben, vorgestellt und diskutiert. Danach werden beide Korpora quantitativ und qualitativ verglichen, wobei zunächst die Effekte ausgewählter Einflussfaktoren auf den Gebrauch der untersuchten Pronomen allgemein überprüft wird, um daraufhin die Rollen und Handlungskategorien im Einzelnen zu vergleichen.

Im Anschluss an den Vergleich der Pronomen wird eine Fallstudie zum ICH-Gebrauch an einem Text unternommen, in dem der Gebrauch der ersten Person Singular deutlich vom Durchschnitt der anderen Texte abweicht. Daran wird gezeigt, dass die zugrundeliegenden Schreibkonventionen nicht deterministisch sind und dass erfahrene Autoren auch dann eine angemessene wissenschaftliche Identität konstruieren können, wenn sie sich nicht an die allgemein verfolgten Regeln halten.

Darauf folgt ein Exkurs zur sozialpsychologischen Perspektive des ICH-Gebrauchs und zeichnet an ausgewählten Beispielen aus dem Korpus nach, wie wissenschaftliche 
Autoren die Strategien der Selbstdarstellung verfolgen, die im Rahmen des Impression Management für Face-to-face-Interaktionen beschrieben wurden (z. B. Eigenwerbung betreiben, sich über Kontakte mit anderen (Wissenschaftlern) aufwerten, Statements vorsorglich abschwächen usw.). Es wird gezeigt, wie sie dies anhand der Sätze, in denen sie über das Pronomen der ersten Person Singular in ihrem Text auftreten, erreichen.

Abschließend wird in Kapitel 6 in einem didaktischen Ausblick diskutiert, wie die Ergebnisse dieser Arbeit für die wissenschaftliche Schreibdidaktik nutzbar gemacht werden können. Auch hier wird wieder die Perspektive vertreten, dass die interaktionale Perspektive berücksichtigt werden muss, indem Studierenden über entsprechende Schreibaufgaben die Möglichkeit gegeben wird, sich in ihrer Rolle als studentischem Autor derjenigen des wissenschaftlichen Autors anzunähern. Kapitel 7 fasst die Ergebnisse dieser Arbeit noch einmal zusammen und gibt einen Ausblick auf mögliche weiterführende Untersuchungen. 


\section{Kapitel 2}

\section{Schreiben als (interkulturelle) Interaktion}

Dieses Kapitel gibt einen Überblick über die grundlegenden theoretischen Ansätze dieser Arbeit. Ich gehe hier insbesondere auf die Zusammenhänge zwischen (wissenschaftlichem) Schreiben, Kultur und Identität ein. Dabei wird Schreiben als sprachliches Handeln aufgefasst, das, genau wie Sprechen, Interaktion in einem sozialen Kontext, in diesem Falle einer Wissenschaftskultur, ist.

Although writing is not face-to-face, it certainly is interaction, in which 'the minute social system' consists of the writer(s) and the reader(s). The expected receivers of language (hearers and readers) influence the choices people make from their array of mediational means. (Ivanič 1998; 99)

Schriftliche Kommunikation ist also in vielen Bereichen vergleichbar mit mündlicher Kommunikation 1 , und dazu zählt auch der soziale Aspekt, so dass es unumgänglich ist, sich in einer Kommunikationssituation eine Identität $\mathrm{zu}$ schaffen, um die eigene Position mit der des Gegenüber in Beziehung zu setzen (s. z. B. Hyland 2005).

Im Folgenden gebe ich einen allgemeinen Überblick über diese grundlegende Notwendigkeit, in einer sprachlichen Interaktion Identitäten und Positionen (sprachlich) zu konstruieren und beziehe dabei Theorien und Ergebnisse aus der Soziologie, der Sozialpsychologie sowie der Linguistik, insbesondere der Gesprächs- und Konversationsanalyse, mit ein, in denen dieses Thema ausführlich untersucht wurde. In diesem Zusammenhang zeige ich konkret, inwiefern diese Ergebnisse für das Schreiben, insbesondere das wissenschaftliche Schreiben, relevant sind. Dabei ist es auch wesentlich, das wissenschaftliche Schreiben in seiner kulturellen Bedingtheit wahrzunehmen. Zu diesem Zweck erläutere ich anschließend die dieser Arbeit zugrundeliegende Auffassung von Wissenschaftskultur, um im dritten Kapitel die Perspektive konkret auf die sprachlichen Mittel der Interaktion, genauer der Identitätskonstruktion, zu lenken, die wissenschaftlichen Autoren in den beiden hier betrachteten Wissenschaftskulturen zur

\footnotetext{
${ }^{1}$ Ein eingängiges Bild dieses grundsätzlich (zumindest in dieser Hinsicht) identischen Charakters von Sprechen und Schreiben hat auch Konrad Ehlich mit seiner Vorstellung des Schreibens als "zerdehnter Sprechhandlungssituation“ (s. z. B. Ehlich 2007. 542) gegeben.
} 
Verfügung stehen. Im dritten Kapitel werden dann auch die wesentlichen sprachwissenschaftlichen Ansätze vorgestellt, aus denen diese Fragestellung in der internationalen Forschung aktuell betrachtet wird.

\subsection{Wissenschaftliches Schreiben als soziale Interaktion}

Over the past decade or so, academic writing has gradually lost its traditional tag as an objective, faceless and impersonal form of discourse and come to be seen as a persuasive endeavour involving interaction between writers and readers. This view sees academics as not simply producing texts that plausibly represent an external reality, but also as using language to acknowledge, construct and negotiate social relations. Writers seek to offer a credible representation of themselves and their work by claiming solidarity with readers, evaluating their material and acknowledging alternative views, so that controlling the level of personality in a text becomes central to building a convincing argument. (Hyland 2005: 173)

Wissenschaftliches Schreiben ist offensichtlich mehr als nur die Darstellung objektiver Gegebenheiten, und unterschiedlichste Faktoren haben Einfluss darauf, wie in den verschiedenen Fach- und Wissenschaftskulturen geschrieben wird und was als „wissenschaftlich“ erachtet wird. Diese Perspektive wird inzwischen immer mehr zum allgemein anerkannten Konsens (z. B. Hyland und Bondi 2004; Fløttum u. a. 2006).

Darüber hinaus erfüllt das wissenschaftliche Schreiben aber innerhalb einer Wissenschafts- bzw. Disziplinkultur auch soziale Funktionen, die über die reine Vermittlung neuen Wissens hinausreichen bzw. diese sogar bedingen (Myers 1989. 5) und die häufig sehr subtil ablaufen. Diese sozialen, rhetorischen und damit interaktiven Funktionen wissenschaftlichen Schreibens sind immer sprach- und natürlich fachkulturell gebunden und spielen wesentlich in die sprachliche Gestaltung eines konkreten Textes hinein. Insbesondere die in dieser Arbeit untersuchten Personalpronomen und ihre Funktionen in Hinblick auf die Konstruktion einer wissenschaftlichen Autorenpersona (Cherry 1988) müssen in erster Linie als derartige sozial-interaktive Elemente betrachtet werden, deren Nutzung sich nicht nur nach rein textsorten- oder wissenschaftsspezifischen Konventionen richtet, sondern auch von übergreifenden Prinzipien der sozialen Interaktion wie z. B. Höflichkeitsregeln beeinflusst werden (können) (s. z. B. Myers 1989).

Dabei ist der Fokus auf sozialer Interaktion in Bezug auf einen geschriebenen wissenschaftlichen Text auf den ersten Blick möglicherweise nicht offensichtlich. Wenn man aber bedenkt, dass natürlich auch geschriebene Sprache in erster Linie der Kommunikation dient und dass das Verfassen wissenschaftlicher Texte an einen sehr spezifischen sozialen Kontext gebunden ist, wird die interaktionale Perspektive deutlich. Dass für 
Kommunikation auch immer Interaktion stattfinden muss, hebt die Sozialpsychologie eindeutig hervor:

In Definitionen von Kommunikation wird meist darauf hingewiesen, daß Kommunikation eine spezifische Form der Interaktion ist, doch hat sich eine Trennung zwischen Interaktion und Kommunikation als willkürlich und deshalb wissenschaftlich unfruchtbar erwiesen. Festzuhalten bleibt allerdings, daß über den Begriff ,Kommunikation' die Interaktion auch die Bedeutung von ,Informationsaustausch' bekommt. Dabei ist wiederum das Wort ,Austausch' zu unterstreichen, um hervorzuheben, daß von Interaktion und Kommunikation immer dann die Rede ist, wenn nicht eine einseitig gerichtete Handlung vorliegt, sondern eine Interdependenz, also eine Wechselwirkung zwischen den beteiligten Personen. (Thomas|1991; 55)

Obwohl die interaktionale Linguistik, die sich mit eben dieser Wechselwirkung in der sprachlichen Kommunikation beschäftigt, sich bisher vorrangig mit Gesprächen in Face-to-face-Interaktionen beschäftigt hat (vgl. z. B. Selting und Couper-Kuhlen 2000), können viele ihrer Prinzipien auf die geschriebene Sprache und damit auf wissenschaftliche Texte übertragen werden (vgl. z. B. Myers 1989, 2-3). Einen detaillierten Forschungsüberblick über die verschiedenen Forschungsrichtungen, die Schreiben als soziale Interaktion betrachten, gibt Triantafillia Kostouli (Kostouli 2005a: 2-3). Sie hebt hervor, dass

[...] writing should be seen as an inherently dialogic and a socially-situated process of making meanings through texts; written texts are not seen as neutral structures produced by autonomous writers but as units of social action conveying ideological meanings. (Kostouli 2005a: 1)

Dennoch gibt es natürlich Spezifika der schriftlichen Kommunikation, die bei einer interaktionalen Analyse wissenschaftlicher Artikel berücksichtigt werden müssen. Beispielsweise stellt sich die Frage, inwieweit in einem schriftlichen Text tatsächlich der oben beschriebene Austausch stattfinden kann, insbesondere, wie die (in der Situation der Textproduktion normalerweise nicht anwesenden) Leser auf die Kommunikation, also den Text, rückwirken können. Myers geht aber dennoch davon aus, dass die geschriebene und die gesprochene Sprache in diesem Aspekt ausreichend vergleichbar sind:

If there is no immediate speaker $(\mathrm{S})$ or hearer $(\mathrm{H}),[\ldots]$ it may not seem to be an example of interaction at all, since the people involved are not present to each other. But I think we can still use some insights from conversational pragmatics as a guide to analysis of written texts. (Myers 1989, 2) 
Aber wie und auf welche Art kann man sich nun eine Interaktion zwischen Autor und Leser eines schriftlichen wissenschaftlichen Textes vorstellen? Beispielsweise muss der Autor eines wissenschaftlichen Textes beim Verfassen dieses Textes immer (mindestens) einen mehr oder weniger spezifischen imaginierten Leser vor Augen haben, an den er seinen Text richtet (vgl. z. B. Hyland (2001b, 2004, 2005), s. auch Kap. 3.2.2). Dies zeigt sich deutlich in verschiedenen Merkmalen. Beispielsweise benötigt der Autor immer einen Referenzleser, an dem er bemisst, was er an Vorwissen voraussetzen kann, was er also wie detailliert beschreiben muss. Hier wird der wechselseitige Austausch besonders deutlich, da erstens der Autor einen Leser vor Augen haben muss und zweitens dessen (antizipierte) Reaktion wiederum Einfluss darauf hat, was der Autor wie darstellt.

Darüber hinaus werden vor allem Schreibanfänger in Schreibanleitungen und -ratgebern häufig darauf hingewiesen, sich vorzustellen, für wen sie ihre Arbeit (in dem Fall meistens eine Hausarbeit oder eine Abschlussarbeit) schreiben (s. z. B. Graefen 2007: 10). Aber auch erfahrene wissenschaftliche Autoren haben offenbar einen Leser vor Augen, wenn sie ihre Texte verfassen, und dieser Leser wird sogar gelegentlich in der zweiten Person angesprochen (s. z. B. Kresta 1995; Hyland 2005; Fløttum u. a. 2006). Dies zeigt, dass man tatsächlich davon ausgehen muss, dass das Schreiben eines wissenschaftlichen Artikels eine interaktive Situation im Thomas'schen Sinne ist, auch wenn der Leser nicht unbedingt eine reale Person sein muss, und dass sich der Autor dessen auch immer (mal mehr, mal weniger) bewusst ist.

Während man bei Face-to-face-Interaktionen im Allgemeinen jedoch recht eindeutig bestimmen kann, wer die relevanten Partner im Rahmen einer Interaktion sind, wer also in welchem Moment als Sprecher und als Hörer fungiert, ist dies in Hinblick auf einen geschrieben Text weit weniger eindeutig zu klären. Zwar wird bei einem wissenschaftlichen Text der Autor bzw. das Autorenteam normalerweise namentlich benannt, so dass eine eindeutige Zuordnung zu den realen Personen unproblematisch erscheint, bei genauerem Hinsehen unterliegt aber gerade ein publizierter Text einer Vielzahl von Überarbeitungsprozessen, bei denen unterschiedliche Personen (beispielsweise Kollegen, Reviewer etc.) Einfluss auf die endgültige Fassung haben (Myers 1989; Jakobs 1997). Und auch die möglichen (realen oder auch imaginierten) Leser sind eine so heterogene Gruppe, dass es zunächst schwierig erscheint, sie konzeptuell greifbar zu machen.

Myers beispielsweise identifiziert zwei wesentliche Lesergruppen, mit denen der Autor in Interaktion tritt und die so den Stil eines wissenschaftlichen Textes beeinflussen. Einerseits ist dies die allgemeine Fachcommunity. Andererseits und deutlich spezifischer sind wissenschaftliche Texte natürlich an diejenigen Forscher gerichtet, die sich mit dem gleichen Forschungsgegenstand und gleichen oder zumindest ähnlichen Fragestellungen auseinandersetzen (Myers 1989: 3). Zwar ist es durchaus vorstellbar, dass es noch weitere Leser oder Lesergruppen gibt, die nicht in die genannten Per- 
sonenkreise fallen, beispielsweise interessierte Laien, Journalisten etc., aber der Autor wendet sich nicht direkt an diese, sondern formuliert seinen Text üblicherweise so, dass er die Bedürfnisse der ersten beiden Gruppen erfüllt. Dies geschieht zum einen natürlich auf inhaltlicher Ebene, vor allem in der Wahl der als bekannt vorausgesetzten Informationen. Zum anderen sieht Myers die Adressierung eines Textes aber auch an anderen Merkmalen manifestiert, die seiner Meinung nach vor allem auf grundlegende Höflichkeitsregeln zwischen den an der Interaktion Beteiligten zurückgeführt werden können (Myers 1989).

Basierend auf der Arbeit von Brown und Levinson (Brown und Levinson 1987) hat Myers Forschungsartikel aus der Molekulargenetik in Hinblick auf Höflichkeitsstrategien, insbesondere die Vermeidung von Face Threatening Acts (FTAs, Gesichtsbedrohungen) ${ }^{2}$ untersucht. Er hat gezeigt, dass diese grundlegenden Mechanismen sozialer Interaktion auch in (natur)wissenschaftlichen Artikeln greifen, obwohl diese nur selten als offenkundig interaktiv wahrgenommen werden (Myers 1989). Dabei gründet er seine Untersuchung auf die Annahme, dass Wissenschaftler in ihren Artikeln Behauptungen (Claims) aufstellen, mit dem Ziel, das Wissen der Community zu erweitern.

Every scientific report states a claim: in other words, it makes a statement that is to be taken as the article's contribution to knowledge. This is the statement that is implied when one cites the article. (Myers 1989. 5)

Diese Claims enthalten entsprechend neues Wissen und richten sich damit möglicherweise gegen bereits bestehendes Wissen innerhalb der Community. Das bedeutet, dass jeder Autor in jeder Publikation potentiell seine Kollegen, d.h. sein relevantes Publikum angreift.

The making of a claim threatens the general scientific audience [...] because it is a demand by individuals for communally granted credit [...]. The claim also threatens the negative face of other researchers [...] because it implies a restriction on what they can do now. (Myers 1989. 5)

Dies ist insofern problematisch, da es genau dieses Publikum ist, das letztlich die im Artikel aufgestellten Claims anerkennen und durch Zitation und Übernahme in die eigene Arbeit zu allgemein anerkanntem Wissen machen soll.

[T]he statement of the individual becomes a fact when it is accepted and used by a consensus of the community [...]. In these interactions, certain FTAs are unavoidable, and must be redressed with various politeness devices. (Myers 1989, 5)

\footnotetext{
${ }^{2}$ Positives und negatives Face definiert er in Anlehnung an Erving Goffman als „roughly the want to be unimpeded and the want to be approved of in certain respects" (Myers 1989. 2), Face Threatening Acts sind entsprechend (sprachliche) Handlungen, durch die das Face der Leser bedroht oder angegriffen werden kann (s. auch Casper-Hehne 2006 83ff.).
} 
Angriffe auf das Face der Leser sind nach Meyers beispielsweise in (Sprech-) Handlungen wie dem Definieren bzw. Benennen neuer Phänomene oder in Spekulationen über bestimmte Zusammenhänge zu sehen. Um diese Angriffe in einer sozial vertretbaren Weise umzusetzen, ist es notwendig, sich als Autor der möglichen FTAs bewusst zu sein und diese so weit wie möglich zu umgehen oder zumindest abzuschwächen. Dies geschieht, indem sich Autoren bestimmter rhetorischer Mittel bedienen, die entweder ihre Solidarität mit ihren Lesern und damit mit der Gemeinschaft betonen, wodurch deren positives Face bestärkt wird, oder die verschiedene Formen des Hedging enthalten, wodurch einerseits die eigenen Claims oder andererseits Angriffe auf die Claims anderer Wissenschaftler abgeschwächt werden. Diese Strategie schützt entsprechend das negative Face der Community-Mitglieder (Myers 1989: 6).

Als Höflichkeitsstrategien, die das positive Face unterstützen, zählt Myers beispielsweise alles, was einen gemeinsamen Ausgangspunkt oder die Annahme gemeinsamen Wissens impliziert. Hier ist für diese Arbeit insbesondere der Gebrauch der ersten Person Plural als inklusives WIR zu nennen. Daneben konnte Myers auch in wissenschaftlichen Texten eher unerwartete Strategien wie das Bekunden von Solidarität durch emotionale Beteiligung (z. B. anhand von Modifizierern wie unexpectedly oder unfortunately) oder sogar Humor (joking) nachweisen (Myers 1989. 7-12).

Bedrohungen für das negative Face begegnen wissenschaftliche Autoren, indem sie auf „klassische“ Elemente des wissenschaftlichen Schreibens zurückgreifen, v. a. auf Hedging und unpersönliche Konstruktionen (Myers 1989: 12). Aber auch die Zuschreibung eines Claims zu einer bestimmten Person bzw. Personengruppe (im Normalfall den Autoren des Textes) kann in diese Kategorie fallen, da jede Form der Personalisierung von Claims diese abschwäche (Myers 1989; 14). An Myers Untersuchung wird also deutlich, dass der Gebrauch von Pronomen eine wesentliche Rolle im wissenschaftlichen Schreiben spielt und nur durch eine interaktive Perspektive sinnvoll betrachtet werden kann.

Und wie immer, wenn eine soziale Interaktionssituation gegeben ist, ist es auch im wissenschaftlichen Schreiben notwendig, sogar unumgänglich, eine Identität zu konstruieren. Der wissenschaftliche Autor hat in diesem Fall, ähnlich wie ein Redner vor Publikum, die Aufgabe, sich verbal darzustellen und das Publikum bzw. die Leserschaft dazu zu bringen, ihn möglichst in dem Licht zu sehen, in dem er sich präsentieren will. Nur so kann er auch seine eigene Arbeit, seine Argumentation und seine Ergebnisse glaubwürdig darstellen und die Leser überzeugen (s. z. B. Hyland 2005; Tang und John 1999; Kuo 1999). Durch die Perspektive auf die soziale Funktion des wissenschaftlichen Schreibens wird also betont, dass z. B. wissenschaftliche Artikel nicht nur der Verbreitung von Wissen dienen, sondern auch und gerade der Positionierung der Autoren.

Gerade die Art und Weise, wie sich ein Autor in seinem Text darstellt, welche Rollen er sprachlich wie übernimmt, wie er seine eigene Position in Bezug auf andere 
Wissenschaftler und deren Ansichten kommuniziert, kurz: wie er seine wissenschaftliche Persona konstruiert, hat einen wesentlichen Einfluss darauf, wie sein Text in der entsprechenden Community aufgenommen wird. Dies äußert sich letztlich nicht selten konkret darin, ob der Text publiziert wird oder nicht (vgl. z. B. Burrough-Boenisch 2003).

Im Folgenden werden nun die grundlegenden Begriffe Identität und Rolle diskutiert. Dazu wird die Beziehung von Identität und Sprache kurz dargelegt und deutlich gemacht, welche Rolle die konstruierte Autorenidentität bzw. die Selbstdarstellung eines wissenschaftlichen Autors in seinem Text spielt.

\subsection{Wissenschaftliches Schreiben und Autorenidentität}

Wer bin ich, wer darf ich sein und wie kann ich das zeigen? Dies sind Fragen, die wir uns in allen möglichen Situationen immer wieder stellen und die auch im wissenschaftlichen Schreiben eine Rolle spielen. Da Sprache eine soziale Eigenschaft ist, liegt eine wesentliche Funktion von Sprache und sprachlicher Interaktion darin, soziale Beziehungen herzustellen und diese zu kommunizieren. Dabei nimmt die Konstruktion sozialer Identitäten und die Positionierung der am (Sprech-)Geschehen beteiligten Individuen eine zentrale Stellung ein. In der Gesprächs- und Konversationsanalyse ist diese Tatsache schon lange bekannt und wurde und wird immer noch umfassend untersucht (vgl. z. B. Androutsopoulos 2001; Lucius-Hoene und Depperman 2004; Bucholtz und Hall 2005, 2008; Llamas und Watt 2010). Und auch in der Analyse von schriftlicher Wissenschaftssprache nimmt diese Perspektive seit ca. 20 Jahren eine immer größere Stellung ein (vgl. z. B. Bazerman 1988; Hyland 2001b, b; Kostouli 2005b). Obwohl die Perspektive auf wissenschaftliches Schreiben als soziale Interaktion, in der die Selbstdarstellung der Autoren und die Konstruktion angemessener wissenschaftlicher Identitäten eine wesentliche Rolle spielt, zwar im internationalen Kontext seit Längerem umfassend untersucht wird (vgl. v. a. Kostouli 2005b), hat sich diese Sichtweise im deutschsprachigen Diskurs noch nicht flächendeckend durchgesetzt. Hier wird als Hauptfunktion wissenschaftlicher Texte und vor allem wissenschaftlicher Artikel immer noch und immer wieder die Funktion der Wissensvermittlung deutlich in den Vordergrund gestellt (s. auch Kap. 3.1. S. 38 und Graefen (1997: 57)). Dies kann man aber auch andersherum sehen: Neues Wissen zu verbreiten ist vielleicht die wichtigste Funktion von wissenschaftlichen Artikeln, aber das geht nur über eine angemessen konstruierte Autorenpersona:

But clearly writers have to [...] say something new [...]. This means demonstrating solidarity with the community and showing respect for its common goals is only part of the story, and that the writers must carefully balance this with vigorous argument for the originality of their claims and by 
the display of an authoritative professional persona. (Hyland (2001b:209), Hervorhebung J. S.)

Es ist also sicherlich mindestens ebenso wichtig, innerhalb des Artikels eine glaubwürdige Persona zu entwickeln, die von der Gemeinschaft als kompetentes Mitglied anerkannt wird, um überhaupt eine Basis für jedwede Argumentation und damit die Verbreitung von neuem Wissen zu haben. Dies ist damit natürlich auch die Grundlage, auf der jede weiterreichende Reputation, die untrennbar mit der wissenschaftlichen Identität des Individuums verknüpft ist bzw. diese sogar maßgeblich ausmacht, aufbauen muss.

Dabei ist auffällig, dass Wissenschaftler offenbar eine sehr genaue, wenn auch meist implizite Vorstellung davon haben, was in ihrer eigenen Wissenschaftskultur für einen wissenschaftlichen Text angemessen ist und was nicht. Dies zeigt sich beispielsweise darin, dass in Experteninterviews (vgl. z. B. Hyland 2001b, 2002a; Steinhoff 2007) regelmäßig Urteile über die „Wissenschaftlichkeit“ der Texte getroffen werden. Einige Texte werden beispielsweise von Steinhoffs Informanten deutlich als "studentisch“ kategorisiert, u. a. basierend auf dem Gebrauch der ersten Person und der dadurch erzeugten Wahrnehmung der Person hinter dem Text durch den Leser (s. z. B. Steinhoff 2007; 176). Und auch im englischsprachigen Bereich wird gerade der Gebrauch von Pronomen mit der Kompetenz des Schreibenden assoziiert, was Hyland hier mit Bezug zu den von ihm untersuchten Studierenden aus Hong Kong bemerkt:

The authorial pronoun is a significant means of promoting a competent scholary identity and gaining acceptance for one's ideas, and while these students were sensitive to its rhetorical effects, they were reluctant to accept its clear connotations of authority and personal commitment. As a result they significantly underused authorial pronouns and determiners, downplayed their role in the research, and adopted a less clearly independent stance compared with expert writers. (Hyland 2002a: 1110)

Dies kann für auch Autoren aus anderen Wissenschaftskulturen zum Problem werden, nämlich z. B. dann, wenn die Schreibkonventionen, die in der eigenen Kultur einen Experten charakterisieren, in der Fremdkultur eher mit einem studentischen Autor assoziiert werden. Insbesondere der tendenzielle Verzicht auf die Selbstreferenz anhand der ersten Person in der deutschsprachigen Schreibtradition, der sich auch in den hier untersuchten Texten zeigt (s. dazu die Auswertung der empirischen Daten in Kap. 5), birgt sicherlich eine Gefahr.

Diverse Untersuchungen haben gezeigt, dass die Schreibkonventionen, die man während der ersten Sozialisation in eine (Wissenschafts-)Kultur gelernt hat, zunächst auf das Schreiben in einer anderen Sprache übertragen werden (s. z. B. Eßer 1997; Flowerdew 1999; Hufeisen 2002; Kaiser 2002; Burrough-Boenisch 2005). Dies könnte in 
diesem Fall dazu führen, dass deutschsprachige Autoren von englischsprachigen Lesern und damit auch Reviewern als "studentischer" wahrgenommen werden, als sie eigentlich sind. Daher ist es gerade (aber nicht nur) für deutschsprachige Autoren, die auf Englisch publizieren wollen (oder müssen), notwendig, die entsprechenden Konventionen zu kennen. Sie müssen wissen, wie sie sich selber als kompetent und glaubwürdig, d.h. wissenschaftlich, in ihren Texten darstellen und u.a. über den Gebrauch von Personalpronomen und die entsprechende Handlungs- und Rollenzuschreibung eine angemessene Autorenidentität konstruieren können.

Hier besteht noch ein großer Forschungsbedarf, da insbesondere die Regeln und Mechanismen, die die Konstruktion von angemessenen wissenschaftlichen Identitäten steuern, stark von der jeweiligen Wissenschaftskultur beeinflusst sind. Da diese Regeln eher subtil und nicht so deutlich erkennbar sind, wie beispielsweise eine andere Textstruktur oder unterschiedliche Zitationsformen, kann man nicht generell davon ausgehen, dass Wissenschaftler, die ihre Arbeiten in einer Fremdsprache publizieren möchten, diese speziellen Regeln auch (er)kennen, selbst wenn sie die jeweilige Fremdsprache (z. B. Englisch) ansonsten sehr gut beherrschen.

Im Folgenden werden die für diese Arbeit grundlegenden Begriffe Identität und (soziale) Rolle diskutiert und operationalisiert. Da dies inhärent sozialwissenschaftliche Konzepte sind, wird hier entsprechend ein Fokus auf Definitionen gelegt, die aus den Sozialwissenschaften stammen. Diese Konzepte sind jedoch bereits mehrfach auch in linguistischen, insbesondere natürlich soziolinguistischen, Arbeiten benutzt worden (vgl. v. a.diverse Arbeiten zur Konstruktion von Identitäten in der Gesprächs- und Konversationsanalyse wie z. B. Androutsopoulos (2001); Benwell und Stokoe (2006); Lucius-Hoene und Depperman (2004)) und haben auch in der Analyse wissenschaftlicher Texte Anwendung gefunden (z. B. Ivanič 1998).

\subsubsection{Identität}

Die Diskussion um den Identitätsbegriff hat eine lange Tradition (vgl. Abels 2006), in deren Verlauf sich unterschiedliche Perspektiven herausgestellt haben, die hier aus Platzgründen nicht alle angesprochen werden können ${ }^{3}$ Im Folgenden wird vor allem eine postmoderne, konstruktivistisch geprägte Sichtweise verfolgt, nach der Identität keine monolithische individuelle Eigenschaft eines Individuums ist, die es hat, und die dieses Individuum von anderen Individuen unterscheidet, sondern in der jeder Mensch viele verschiedene Identitäten hat, die abhängig vom jeweiligen Kontext in sozialen Interaktionen immer wieder neu konstruiert werden.

Durch die postmoderne Perspektive werden also auch in Bezug auf Identitäten die Grundkonzepte von Heterogenität, Vielfalt und Gleichzeitigkeit (Kresic 2006: 106ff.)

\footnotetext{
${ }^{3}$ Für einen Überblick verschiedener Identitätstheorien mit einem Fokus auf Sprache s. z. B. Kresic (2006)
} 
betont und, in Anlehnung an die erkenntnistheoretische Perspektive des Konstruktivismus, die Identitätsarbeit und die soziale Interaktion als deren Ausgangspunkt in den Fokus gestellt (vgl. Kresic 2006. Kap. 6 und 7). Identität ist situationsspezifisch und wird immer erst durch Interaktion erzeugt. Dabei hebt Kresic auch den ,zentralen Stellenwert von Sprache und sprachlicher Interaktion im Hinblick auf Identitätskonstruktion unter den gesellschaftlichen Bedingungen der Postmoderne" hervor (Kresic 2006 130). Entsprechend wird auch in dieser Arbeit davon ausgegangen, dass Identitäten in sozialen Interaktionen immer neu (sprachlich) konstruiert werden und ein einzelnes Individuum über viele verschiedene Identitäten verfügt, die es je nach Kontext und Notwendigkeit herausstellen oder zurückhalten kann (vgl. dazu auch Kresic (2006)).

Diese verschiedenen Identitäten sind also abhängig von den unterschiedlichen sozialen Kontexten, in denen ein Mensch sich befindet, und werden darüber hinaus auch nach individueller Vorliebe bzw. Persönlichkeit konstruiert. Dabei wird deutlich, dass der Begriff Identität nicht nur eine individuelle Komponente beinhaltet, sondern auch eine kollektive. Die möglichen Identitäten, die eine Person annehmen kann, sind gesellschaftlich bedingt, ebenso wie die verbalen und nonverbalen Mittel, mit denen sie diese Identitäten kommunizieren kann. Identität ist nach Ken Hyland (2009. 25) „,something that we actively and publicly accomplish through discourse, and so oriented to community as much as individuality".

Identität ist daher auch eng verbunden mit der sozialen Rolle, die man in einer bestimmten Situation einnimmt, umfasst dabei aber noch mehr. Man kann also in einer Interaktion die Rolle Autofahrer, Fußgänger, Kunde, Restaurantbesucher o. ä. haben. Diese Rolle ist durch den sozialen Kontext vorgegeben und die Rollenschemata und Frames (vgl. z. B. Tannen 1985), die einen bestimmten Handlungsrahmen definieren, innerhalb dessen die Rolle realisiert werden muss, sind kulturell bedingt. Die eigene Identität spielt jetzt insofern in die Realisierung der Rolle hinein, als dass man immer auch individuell die Möglichkeit hat, die Rolle innerhalb des kulturellen Rahmens auszugestalten. Ich kann also ein aggressiver oder ein defensiver Autofahrer sein, ich kann als Gast im Restaurant höflich oder unhöflich sein, je nachdem, was ich individuell, bewusst oder unbewusst, auswähle. Dabei stelle ich mich immer auch selbst dar. Diese Selbstdarstellung und Kommunikation von persönlicher Identität im Rahmen eines kulturell bereitgestellten Rollenslots findet unumgänglich in jeder Interaktion statt und ist Grundbedingung für das gegenseitige Verstehen (Mummendey 2002: 213).

Entsprechend notwendig ist die Kommunikation von Identitäten, Rollen und Positionen auch im wissenschaftlichen Schreiben, wobei hier die wissenschafts- und disziplinspezifischen Identitäts- und Rollenslots zu besetzen sind, die sich von denen im Alltagsleben unterscheiden. Aber auch hier gibt es grundsätzlich vorgegebene Schemata und Frames, die die zu erwartenden Rollenslots sowie die (sprachlichen) Mittel, mit denen sie umgesetzt werden können, bestimmen, welche ihrerseits wiederum in- 
dividuell realisiert werden müssen. Hier sind gerade zwischen dem deutschen und dem englischen bzw. US-amerikanischen wissenschaftlichen Schreiben Unterschiede erwartbar 4

Im Folgenden wird nun kurz der Zusammenhang zwischen Identität und Sprache erläutert und es werden die wesentlichen Grundannahmen zur Identitätskonstruktion im wissenschaftlichen Schreiben dargestellt. Im Anschluss daran wird der Begriff der sozialen Rolle thematisiert.

\subsubsection{Identität und Sprache}

In der Soziolinguistik, insbesondere in der Gesprächs- und Konversationsanalyse spielt das Thema Identität schon lange eine wichtige Rolle. Im klassischen Sinne geht man nach Androutsopoulos (2001: 63) davon aus, dass es eine direkte Beziehung gibt zwischen sozialer Identität und dem Gebrauch spezifischer sprachlicher Mittel bzw. sprachlicher Varianten.

Ein Sprachmerkmal ,signalisiert' z. B. regionale Identität, wenn es signifikant häufiger im Sample der Sprecher aus der fraglichen Region auftritt [...], oder höhere Loyalität zu den Normen einer, vernacular culture' geht mit stärkerer Verwendung grammatischer Nonstandardmerkmale einher [...]. (Androutsopoulos 2001: 63)

In Hinblick auf die postmoderne Sichtweise eines diskursiven Verständnisses von Identität muss diese Grundannahme dahingehend erweitert werden, dass jeder Sprecher aufgrund seiner Zugehörigkeit zu verschiedenen Gruppen über ein größeres Repertoire an sprachlichen Mustern verfügt, aus dem er in jeder Situation gezielt Muster auswählen und kombinieren kann. So kann er stets seinen individuellen „identity claim" (Androutsopoulos 2001, 63) und seine mehrfachen sozialen Zugehörigkeiten zum Ausdruck bringen. Darüber hinaus ist die soziale Bedeutung einzelner sprachlicher Muster und Merkmale nicht als so fest und vorgegeben anzusehen, wie es in der o. g. klassischen Perspektive den Anschein hat. Diese Bedeutung wird vielmehr in jeder Interaktion neu konstruiert und muss von den an der Interaktion Beteiligten jeweils neu interpretiert und konstituiert werden (Androutsopoulos 2001: 63). Hier wird deutlich, dass unterschiedliche soziokulturelle Symboliken und die damit zusammenhängende Auswahl unterschiedlicher Kontextualisierungshinweise, basierend auf unterschiedlichen soziokulturellen Zugehörigkeiten der Interaktionspartner, schnell zu

\footnotetext{
${ }^{4}$ Obwohl man sicherlich grundsätzlich davon ausgehen muss, dass es auch im wissenschaftlichen Schreiben Unterschiede zwischen den verschiedenen Varietäten des Englischen gibt, scheinen diese Unterschiede doch nicht sehr gravierend (Fløttum u.a. 2006 18). Die Ergebnisse von Sanderson (2008a) haben beispielsweise gezeigt, dass zumindest in Bezug auf die Selbstdarstellung der Autoren in wissenschaftlichen Texten, insbesondere den Gebrauch der Pronomen der ersten Person, keine signifikanten Unterschiede zwischen Texten britischer und US-amerikanischer Autoren bestehen.
} 
unterschiedlichen Konstruktionen bzw. Interpretationen der jeweiligen Identität führen können (vgl. auch das Problem der doppelten Kontingenz, Kap. 2.2.2).

Wichtige Grundlagen zu dieser Sichtweise wurden von Robert Le Page und Andrée Tabouret-Keller mit ihrem Ansatz der acts of identity (Le Page und Tabouret-Keller 1985) gelegt. Sie sehen den wichtigsten Antrieb für die Gestaltung des individuellen Sprachgebrauchs in dem Wunsch des Individuums, „sich mit einer oder mehreren Gruppen zu identifizieren oder sich davon zu distanzieren" (Androutsopoulos 2001 64), indem es seine soziale Identität und seine sozialen Rollen sprachlich konstruiert. Dabei müssen vier Bedingungen erfüllt sein bzw. das Individuum muss über vier Eigenschaften verfügen (Androutsopoulos 2001; 64):

- die Fähigkeit, die jeweilige soziale Gruppe zu identifizieren,

- den Zugang zu dieser Gruppe und die Fähigkeit, deren Sprachverhalten zu analysieren,

- die Motivation, sich dieser Gruppe anzuschließen oder sich von ihr zu distanzieren,

- die Fähigkeit, das eigene sprachliche bzw. kommunikative Verhalten anzupassen.

Diese Interdependenz zwischen der Identifizierung des Individuums mit einer bestimmten Gruppe und seinem sprachlichen Verhalten führt zum Phänomen der Fokussierung, die Androutsopoulos als „die Ausprägung von Sprachnormen im Rahmen einer sozialen Gruppe“ beschreibt (Androutsopoulos (2001: 64), nach Le Page und Tabouret-Keller (1985: 187)):

Fokussierung wird gefördert von der engen und täglichen Interaktion zwischen den Gruppenmitgliedern, dem Gefühl eines gemeinsamen Ziels und der Nachahmung von gruppenspezifisch relevanten Vorbildern [...]. Sprachlich bedeutet Fokussierung die Ausprägung von Ähnlichkeiten zwischen den Mitgliedern der Gruppe und demzufolge eine Abnahme von Variabilität im Sprachgebrauch dieser Gruppe. (Androutsopoulos 2001: 64)

Dies ist letztlich als die soziolinguistische Grundlage derjenigen Prozesse zu betrachten, die zur Ausdifferenzierung unterschiedlicher Register und Varietäten von der Jugendsprache bis hin zu den verschiedenen Fach- und Wissenschaftssprachen führen, und leitet auf die grundlegende Fragestellung dieser Arbeit hin, wie ein individueller Autor die Pronomen der ersten Person in seinem Text benutzen kann, um seine Zugehörigkeit zu der Gruppe der wissenschaftlichen Autoren zu kommunizieren und insbesondere die eigene Identität innerhalb der vorgegebenen soziokulturellen Parameter angemessen darzustellen. 


\subsubsection{Identität und Selbstdarstellung im wissenschaftlichen Schreiben}

Jeder Wissenschaftler stellt sich zwangsläufig in seinem Text auf die eine oder andere Weise dar, so wie in jeder anderen Interaktion auch immer die jeweiligen Identitäten, Rollen und Positionen bewusst oder unbewusst kommuniziert werden. Während man jedoch in einer Face-to-face-Interaktion immer auch nichtsprachliche Mittel und Medien zur Verfügung hat - von Gestik und Mimik über Kleidung, Körpersprache zur Stellung im Raum etc. - , so hat man beim Schreiben natürlich hauptsächlich sprachliche Mittel zur Hand, um etwas zu kommunizieren. So müssen entsprechend auch die eigene Rolle und der eigene Standpunkt (stance, vgl. Hyland (2001a, 2005, 2008)) verbal kommuniziert werden.

If the self is not a fixed pre-language entity, then writers can be sensitized to the possibilities of inventing their 'selves' through their writing. They can, as it were, break free from the real or imagined moulds of behaviour imposed upon them by their discourse situations to inhabit different, chosen roles in their writing. (Tang und John 1999: 24)

Ein wissenschaftlicher Autor hat also viele unterschiedliche Möglichkeiten, in einem Text seine Identität zu konstruieren5(s. v. a. Kap. 3.3). Neben dem Gebrauch der ersten Person, die wohl die eindeutigste Möglichkeit ist, wurden vor allem folgende Mittel untersucht: epistemic modality, argumentative connectives, negative und concessive constructions, selected lexemes und metatextual expressions (Gotti 2009a). Eine der wichtigsten Untersuchungen zur Bedeutung der Identität im wissenschaftlichen Schreiben stammt von Roz Ivanič (Ivanič 1998). In ihrer Untersuchung von acht "mature students“, also von Studierenden, die sich erst spät zu einem Universitätsstudium entschlossen haben und praktisch „auf dem zweiten Bildungsweg“ studieren, vertritt sie ebenfalls einen sozialkonstruktivistischen Standpunkt, d. h. sie geht davon aus, dass Identitäten auch im wissenschaftlichen Schreiben immer neu und abhängig vom soziokulturellen Kontext konstruiert werden.

Konkret hat sie drei verschiedene Identitäten (Selves) herausgearbeitet, die wissenschaftliche bzw. studentische Autoren innehaben (können). Diese sind im Prinzip drei Teile der individuellen Persönlichkeit eines Autors, die sich in seinem Text wiederfinden lassen, und Ivanič verwendet äquivalent zu "self“ auch den Begriff „identity“ (Ivanič 1998: 24). Diese drei „selves/Selbste“ nennt sie das autobiografische Selbst (autobiographical self), das diskursive Selbst (discoursal self) und das Selbst als Autor (self as author).

\footnotetext{
${ }^{5}$ Das Konzept „Identität“ spielt im wissenschaftlichen Schreiben auch weiterreichend eine wesentliche Rolle, nicht immer geht es nur um die Identität des jeweiligen Autors, sondern oft auch um die Identität derjenigen, deren Ideen und Ergebnisse in der jeweiligen Arbeit verwendet werden. Ihre Identität muss auf jeden Fall offengelegt und geschützt werden, nicht umsonst gilt das Plagiat als schweres Vergehen im wissenschaftlichen Betrieb (vgl. z. B. Ivanič 1998: 3).
} 
Dabei versteht sie unter dem autobiographischen Selbst das, was man am ehesten mit der tatsächlichen Persönlichkeit des Autors assoziieren könnte (wenn es so etwas denn gibt). Das autobiographische Selbst basiert auf den individuellen, idiosynkratischen Erfahrungen der jeweiligen Person und repräsentiert die sozial konstruierte und sich ständig ändernde Identität, die jeder Autor überwiegend unbewusst in jeden seiner Schreibprozesse mit einbringt (vgl. Ivanič 1998: 24-25).

Das diskursive Selbst dagegen stellt den Teil des Selbst dar, den ein Autor - bewusst oder unbewusst - in einem bestimmten Text vermittelt (Ivanič 1998: 25). Dieses Selbst wird durch die bestimmten diskursiven Eigenschaften eines Textes erzeugt, die ihrerseits abhängig sind von den Werten, Vorstellungen und Machtrelationen innerhalb des sozialen Kontextes, in dem der Text entsteht. So kann sich das diskursive Selbst eines wissenschaftlichen Autors in einem Forschungsartikel deutlich von demjenigen des gleichen Autors in einer Einführung oder einer Rezension unterscheiden.

Das Selbst als Autor schließlich repräsentiert das Ausmaß, in dem der Autor sich selbst als Quelle des Textes darstellt. Hier trifft der Autor Entscheidungen, wie er seine Haltungen und Einstellungen rhetorisch vertritt, welche Position er wie sprachlich einnimmt, wie autoritativ er Meinungen und Überzeugungen äußert und ob und wie er sich selbst als verbindliche und maßgebliche Instanz darstellt (authoritativeness). Dabei steht jedem wissenschaftlichen Autor eine Bandbreite an rhetorischen Möglichkeiten zur Auswahl.

The self as author is particularly significant when discussing academic writing, since writers differ considerably in how far they claim authority as the source of the content of the text, and in how far they establish an authorial presence in their writing. Some attribute all the ideas in their writing to other authorities, effacing themselves completely; others take up a strong authorial stance. Some do this by presenting the content of their writing as objective truth, some do it by taking responsibility for their authorship. (Ivanič 1998, 26)

Dabei wird das Selbst als Autor einerseits von den persönlichen Erfahrungen und Einstellungen des "tatsächlichen“ Menschen beeinflusst, d.h. vom autobiographischen Selbst, andererseits hat natürlich auch das diskursive Selbst und damit einhergehend der (engere) soziale Entstehungskontext eines Textes Anteil an der Konstruktion des Selbst als Autor.

Neben diesen drei Aspekten von Autorenidentität (writer identity) haben nach Ivanič auch die vom soziokulturellen, hier vor allem vom disziplinären Kontext vorgegebenen possibilities for selfhood, die Auswahl an institutionell vorbestimmten Identitäten (Ivanič 1998, 27), Einfluss auf die Selbstdarstellung von wissenschaftlichen Autoren 
in ihren Texten. Diese sieht sie als vierten Aspekt der writer identity. ${ }^{6}$ Der disziplinäre bzw. soziale Kontext stellt entsprechend prototypische Identitätsslots zur Verfügung, d.h.

,social' identities in the sense that they do not just belong to particular individuals. In any institutional context there will be several socially available possibilities for self-hood: several ways of doing the same thing. Of these some will be privileged over others, in the sense that the institution accords them more status. (Ivanič 1998: 27)

Dieser vierte Aspekt von Identität umfasst also die Möglichkeiten der Selbstdarstellung, die der soziokulturelle und institutionelle Rahmen, in dem der Text produziert wird, bietet, wobei offenbar einige dieser Möglichkeiten mehr Prestige haben und insofern „wertvoller“ sind als andere. Entsprechend besteht für wissenschaftliche Autoren im Rahmen dieses Kontextes der Druck, sich möglichst der eher prestigeträchtigen Möglichkeiten der Selbstdarstellung zu bedienen. Dies setzt die Kenntnis der jeweiligen Möglichkeiten und ihrer entsprechenden Konnotationen voraus. Hier liegt offensichtlich eine Schwierigkeit für noch nicht erfahrene Autoren und Autoren, die in einem anderen soziokulturellen Wissenschaftskontext sozialisiert wurden.

\subsubsection{Rolle}

Ähnlich wie das Konzept der Identität ist auch der Begriff der Rolle alles andere als unumstritten und wird im sozialwissenschaftlichen bzw. soziologischen Kontext immer noch und immer wieder diskutiert (s. z. B. Gerhardt 2008). Er steht seit längerem im Fokus verschiedener Wissenschaftler, die unterschiedliche theoretische Perspektiven und Strömungen vertreten, und hat sich in der Geschichte des Faches kontinuierlich weiterentwickelt (für einen Überblick s. z. B. Abels 2009. Kap. 3). Da es nicht Ziel dieser Arbeit ist, einen umfassenden Überblick über den sozialwissenschaftlichen Rollenbegriff zu geben, werden hier, ebenso wie beim Begriff Identität, nur diejenigen Aspekte angesprochen, die für diese Arbeit relevant sind. Insbesondere werden solche Ansätze hervorgehoben, die sich explizit mit der Beschreibung und Umsetzung sozialer Identitäten und Rollen im wissenschaftlichen Schreiben beschäftigen. Dabei ist zu beachten, dass der Rollenbegriff auch in der Linguistik angewandt wird (z. B. semantische Rollen), dass hier aber der sozialwissenschaftliche Begriff im Vordergrund steht, da das wissenschaftliche Schreiben als soziale Interaktion betrachtet wird. Im Folgenden werden also kurz die soziologischen Hintergründe des Konzepts Rolle vorgestellt um danach die soziolinguistische Perspektive auf das wissenschaftliche Schreiben zu betrachten.

\footnotetext{
${ }^{6}$ Es ist zu bedenken, dass diese vier Aspekte der Identitätskonstruktion nach Ivanič nicht separat und unabhängig voneinander zu betrachten, sondern intensiv miteinander verflochten und interdependent sind.
} 
Soziale Situationen unterscheiden sich danach, wie stark sie strukturiert sind, d. h. wie eindeutig und zuverlässig die in ihnen enthaltenen Interpretationskontexte gestaltet sind. Diese Kontexte oder auch Schemata sind gesellschaftliche Regeln, die bestimmte Situationen bzw. deren Ablauf und die Handlungsoptionen vorstrukturieren. Dadurch wird das Problem der doppelten Kontingenz in diesen Situationen umgangen. Kontingenz im soziologischen Sinne beschreibt vereinfacht dargestellt die Situation, dass etwas sein oder eintreten kann, aber nicht muss.

Kontingenz bezeichnet zunächst Abhängigkeit und somit Bestimmtheit eines Sachverhalts oder einer Situation, meint ferner aber auch, dass der Sachverhalt bzw. die Situation anders sein könnte, als sie gerade ist. (Vester 2009. 50)

Kontingenz entsteht dadurch, dass in jeder Interaktionssituation die beteiligten Personen von ihrem Gegenüber (oder ihren Gegenübern) ein bestimmtes Verhalten erwarten, aber nicht wissen, ob sich das Gegenüber auch tatsächlich so verhalten wird. Doppelt wird die Kontingenz dadurch, dass man auch Erwartungen bezüglich der Erwartungen hat, die das Gegenüber an einen selbst stellt (Vester 2009. Kap. 3.3). Diese „Erwartungserwartungen“ (Vester 2009: 50) können nun ihrerseits zutreffen oder nicht, wobei jeder der Beteiligten an alle anderen Beteiligten seine eigenen Verhaltensund Erwartungserwartungen stellt.

Schemata lösen diese Komplexität zumindest teilweise auf, indem sie den interagierenden Personen Regeln an die Hand geben (oder auch aufzwingen), die die gegebene Interaktionssituation strukturieren. Dadurch werden bestimmte Handlungen und Erwartungen wahrscheinlicher als andere. Die interagierenden Personen erhalten damit bestimmte soziale Rollen.

Durch soziale Rollen werden die Unsicherheiten, die sich auf Grund der doppelten Kontingenz in Handlungssituationen ergeben, verringert. Die soziale Begegnung von Ego und Alter wird durch Rollen strukturiert und wechselseitiges Rollenverständnis vorausgesetzt - erleichtert. (Vester 2009: 52)

Soziale Rollen werden hier also verstanden als vorgegebene Verhaltensmuster. Im gesellschaftlichen Kontext wird von den Inhabern einer Rolle erwartet, dass sie diese Verhaltensmuster erfüllen. Grundsätzlich ist jedoch zu sagen, dass diese Verhaltensmuster generell nicht so starr und fixiert sind, wie es hier möglicherweise scheint. So ist davon auszugehen, dass jede Person eine in gewissem Maße idiosynkratische Vorstellung davon hat, wie eine spezifische Rolle umzusetzen ist. Entsprechend sind sowohl die Realisierungen als auch die Erwartungen, die an die jeweilige Rolle bzw. den Rolleninhaber gestellt werden, nie vollständig deckungsgleich.

Dass die Individualität des Einzelnen beim Umsetzen sozialer Rollen immer mit hineinspielt, betont auch Uta Gerhardt (Gerhardt 2008, 386). Für sie stellt die soziale 
Rolle „ein Schema zur Deutung von Handlungsakten bereit [...], das den Spielraum akzeptierten Verhaltens für Personen in Interaktionsbeziehungen festlegt " (Gerhardt 1971: 226) und sie definiert sie folgendermaßen:

Rollen sind das Ergebnis einer Abstraktion, welche Haltungen, Eigenschaften, Leistungen und Tätigkeiten für gleichrangige und/oder gleichartige Personen zu einem aus Handlungsregeln bestehenden Typisierungsschema zusammenfasst. (Gerhardt 1971; 226)

Soziale Rollen sind also als Handlungsregeln zu verstehen, die sich zu einem Handlungs- und Verhaltensschema verdichtet haben. Um sie empirisch zu untersuchen, ist es notwendig, das Verhalten und insbesondere die Handlungen von Personen zu betrachten, die sich bestimmte Rollen zuschreiben ${ }^{7}$

Gerhardt weist darauf hin, dass Rollen immer an einen spezifischen soziokulturellen Kontext gebunden sind, innerhalb dessen sie normativ wirken können und sollen (Gerhardt 2008: 387). Diese Kontexte sind jedoch variabel und können sich im Lauf der Zeit verändern, so dass sich die Rollen und die mit ihnen verbundenen Handlungsregeln ebenfalls ändern können. Darüber hinaus postuliert sie „drei Ebenen der Abstraktion von der unmittelbaren Erlebniswelt" (Gerhardt 2008: 387), auf denen sich diese strukturellen Kontexte auswirken können, nämlich den Status, die Position und die Situation. Sie differenziert folglich zwischen Statusrollen, Positionsrollen und Situationsrollen, die sich hinsichtlich ihrer Festigkeit und Kohärenz, d. h. ihrer Institutionalisiertheit, unterscheiden. Als Statusrollen fasst sie diejenigen Rollen auf, die innerhalb des jeweiligen soziokulturellen Kontextes am stärksten institutionalisiert bzw. gesellschaftlich geformt sind. Hierunter fallen Rollen, die an Merkmale wie Alter, Geschlecht, Ethnie oder Nationalität gebunden sind (Gerhardt 2008. 387). Auf mittlerer Ebene siedelt sie die Positionsrollen an, unter die sie beispielsweise Berufsrollen fasst (Gerhardt 2008: 388). Situationsrollen hingegen sind am wenigsten im Rahmen des soziokulturellen Kontextes institutionalisert, hierunter fallen Rollen wie „Freund“, „Gast" etc. (Gerhardt 2008. 388).

Unabhängig von ihrer institutionellen Festigkeit sind diese Rollen aber, wie oben schon beschrieben, immer auch interpretierbar und sie werden situativ immer neu interpretiert. In jeder Situation spielen immer verschiedene Rollen auf unterschiedlichen Abstraktionsniveaus zusammen, was bedeutet, dass neben der jeweiligen Interpretation von relativ stark fixierten Rollen auch immer ein situationsgebundenes Aushandeln

\footnotetext{
${ }^{7}$ Diese Handlungen können sprachlicher, aber auch nichtsprachlicher Natur sein, was sich in den hier untersuchten Texten eben in sprachlichen Handlungen (wie z. B. metatextuellen Hinweisen) oder in versprachlichten Handlungen zeigen kann. Als letztere bezeichne ich diejenigen Handlungen, die zwar in der realen Umsetzung nicht (unbedingt) sprachlich ausgeführt werden, die aber natürlich in Rahmen der Berichterstattung in wissenschaftlichen Texten sprachlich dargestellt werden müssen, beispielsweise das Durchführen bestimmter methodischer Schritte wie das Berechnen von Korrelationen etc. (s. auch Kap. 3.3.2. S. 71 .
} 
eines angemessenen rollenspezifischen Verhaltens stattfindet. Dieses Aushandeln setzt entsprechend immer mindestens zwei Handelnde voraus, mit anderen Worten, soziale Rollen können ausschließlich in Interaktion realisiert werden bzw. haben nur in Interaktion Relevanz. Dabei betont Gerhardt jedoch, dass das Gegenüber sowohl bestimmt als auch unbestimmt sein kann (Gerhardt 2008 387), wobei gerade im wissenschaftlichen Schreiben ein unbestimmtes oder zumindest nicht vollends bestimmtes Gegenüber die Regel ist.

Darüber hinaus hat auch jeder Rolleninhaber die Freiheit, sich bewusst gegen die gesellschaftlich geforderten Verhaltensweisen seiner Rolle zu stellen, wodurch er unterschiedliche Dinge symbolisieren kann. Erving Goffman nennt dies „Rollendistanz" (Goffman 1961, 1973) 8 Diese Möglichkeit, sich von der eigenen Rolle zu distanzieren, wird auch dadurch begünstigt, dass sich jeder Mensch im Laufe seines Lebens ein Rollenrepertoire aneignet, aus dem heraus er sich in jeder Situation die für ihn jeweils passende (Kombination aus verschiedenen) Rolle(n) auswählen kann.

Der Verhaltensakt ist der Schauplatz gleichzeitiger Einwirkungen heterogener Status-, Positions- und Situations-Rollen auf das Handeln, und deren je aktuelles Zusammenspiel ist ein Moment der individuellen Identität. Die Je-Einmaligkeit sowohl des einzelnen Verhaltensaktes wie auch der Individualität als dem Ausdruck einer besonderen Aggregation von Rollen kann daraus hergeleitet werden. (Gerhardt 1971; 296)

Soziale Rollen sind also immer an den soziokulturellen Kontext, in dem sie realisiert werden, gebunden und können gemeinsam mit diesem variieren. Darüber hinaus ist ihre eigentliche Funktion zwar, dass sie grundlegende Regeln bündeln, wie die jeweiligen Rolleninhaber innerhalb des spezifischen Kontextes zu handeln bzw. sich zu verhalten haben. Allerdings sind diese Regeln nicht absolut deterministisch, sondern die reale Ausgestaltung einer Rolle in einer spezifischen Situation ist abhängig von dem sie verkörpernden Individuum. Jeder Mensch hat als Teil verschiedener Gruppen mit unterschiedlichen Rollen immer - zumindest bis zu einem gewissen Grad - die Wahl, wie er sich als Inhaber einer bestimmten sozialen Rolle darstellen will und sogar, ob er diese Rolle in der betreffenden Situation überhaupt übernehmen will.9 ${ }^{9}$ Diese Grundannahmen fasst Gerhardt in vier Hypothesen zusammen:

\section{Rollen haben einen strukturellen Kontext.}

\footnotetext{
${ }^{8}$ Ein Grund, warum sich Personen von ihrer Rolle distanzieren, kann sein, dass eine Rolle hohe Anforderungen an ihren Inhaber stellt und der Inhaber so die Möglichkeit hat, sich von dem damit verbundenen Stress zu befreien (Vester 2010 27). Als Beispiel nennt Vester Chirurgen, die bei einer Operation Scherze machen und nicht-medizinische Gespräche führen, um damit deutlich zu machen, dass sie souverän mit den Anforderungen ihrer Rolle umgehen können (Vester 2010: 27).

${ }^{9}$ Dies gilt natürlich auch andersherum. Jedem Individuum werden in einer realen sozialen Situation von anderen Individuen Rollen zugeschrieben, die nicht unbedingt mit denjenigen übereinstimmen müssen, die diese Person sich selber zuschreibt. Zudem kann auch das Gegenüber bewusst oder unbewusst entscheiden, ob es die Rolle, die sich jemand selber zuschreibt, akzeptiert oder nicht.
} 
2. Die strukturellen Kontexte variieren im Grad der Abstraktion von der unmittelbaren Erlebniswelt sowie im Grad der Generalisierung ihrer Gültigkeit.

3. Entsprechend ihrem strukturellen Kontext entstehen Rollen von unterschiedlichem Grad der institutionellen Verfestigung in einer Gesellschaft.

4. Rollen und strukturelle Kontexte, sofern sie handlungsrelevant werden, werden interpretiert. (Gerhardt 2008: 387)

Vergleichbare Kategorisierungen wie Gerhardts drei Abstraktionsebenen von Rollen, die Status-, Positions- und Situations-Rollen, finden sich auch in Untersuchungen zum wissenschaftlichen Schreiben. Ramona Tang und Suganthi John beispielsweise haben ebenfalls drei Rollenkategorien charakterisiert, die sich in ihrem jeweiligen Skopus, d.h. in der Reichweite, in der sie die Identitäten einer betreffenden Person prägen, unterscheiden. Diese nennen sie soziale Rollen (societal roles), Diskursrollen (discourse roles) und Genre-Rollen (genre roles) (Tang und John 1999: 25).

Soziale Rollen haben dabei die größte Reichweite und sind innerhalb der Persönlichkeit eines Menschen angesiedelt, beispielsweise die Rollen „Vater", „Tochter", "Amerikaner" etc. (Tang und John 1999. 25). Damit sind sie vergleichbar mit Gerhardts Statusrollen. Diskursrollen sind dagegen schon spezifischer auf bestimmte Situationen und Diskurskontexte beschränkt, häufig im Rahmen beruflicher Diskurse (z. B. die Rollen Anwalt - Klient oder Arzt - Patient). Dabei sind unter bestimmten Umständen die Übergänge jedoch fließend. Diskursrollen lassen sich daher weitestgehend mit den von Gerhardt definierten Positionsrollen korrelieren (Gerhardt 2008, 387).

Generally, these roles only hold within the confines of that discourse community. For example, a person is not 'a client' outside of the courtroom or the lawyer's office. In some cases, however, a person's job might come to define him or her so well that the society defines them by it. When this happens, what began as a discourse role might develop into a societal role, as when a prominent medical doctor is identified as such even when he or she is picking up a head of lettuce at the supermarket. (Tang und John|1999. 25)

Genre-Rollen dagegen sind auf ein spezifisches Genre abgestimmt und damit in einer Diskursgemeinschaft sowie auch einer spezifischen Diskurssituation verortet. Diese Rollen sind sehr eng mit dem jeweiligen Genre verbunden und der Übergang zu einer sozialen Rolle (im engeren Sinne Tang/Johns) ist nicht wahrscheinlich.

[I]n this article, when we speak of the writer as a guide through the essay or the architect of the essay, we are discussing genre roles, identities that are created within the genre of the undergraduate academic essay or academic 
research article. A genre role will generally not develop into a societal role. One does not identify a person as a guide through the essay in any context outside of academic writing. (Tang und John 1999: 25)

Dabei ist zu beachten, dass alle drei Rollenkategorien zumindest theoretisch in schriftlichen Texten, auch im wissenschaftlichen Schreiben, sprachlich dargestellt werden können. Die Ergebnisse dieser und weiterer Untersuchungen zu Genre-Rollen im wissenschaftlichen Schreiben werden in Kapitel 3.3.2 diskutiert.

Es ist in diesem Abschnitt deutlich geworden, dass soziale Rollen zwar als soziokulturell und situational vorgegebene Verhaltensmuster wirken, dabei aber durchaus individuell variabel sein können. Da sich die Rollenübernahme in der Interaktion vor allem daran zeigt, wie die Beteiligten handeln, liegt in der Auswertung der Korpustexte der Fokus auf den Handlungen, die sich die Autoren durch die Pronomen der ersten Person selber zuschreiben, wo sie also explizit von sich sagen: „ICH bin derjenige der XY tut/getan hat."

\subsubsection{Wissenschaftskultur und Diskursgemeinschaft}

Dass es so etwas wie Wissenschaftskultur oder kulturell unterschiedliche wissenschaftliche Stile gibt, hat sich spätestens nach Johan Galtungs einflussreichem Essay über „sachsonische, teutonische, gallische und nipponische Wissenschaft" (Galtung 1985) durchgesetzt. Aber was genau unter Wissenschaftskultur zu verstehen ist, inwiefern Faktoren wie Nation, Sprache, Disziplin usw. Einfluss haben bzw. als eigenständige Kulturen angesehen werden müssen, ganz davon abgesehen, was unter dem Begriff Kultur an sich zu verstehen ist, sind Fragen, die sich immer wieder stellen und für jede Forschungsarbeit individuell neu beantwortet werden müssen. Daher soll in diesem Abschnitt ein kurzer Überblick folgen, wie Wissenschaftskultur in dieser Arbeit gefasst wird, d. h. welche Konzepte und Einflussfaktoren im Vordergrund stehen und wie sie auf die Zusammenstellung des Datenkorpus gewirkt haben.

In den vorhergehenden Teilen dieses Kapitels ist deutlich geworden, dass Menschen ihre Identitäten in sozialen Interaktionen konstruieren, indem sie u. a. Rollen übernehmen und Handlungen ausführen $[10$, und dass dies immer innerhalb von sozialen und kulturellen Kontexten stattfindet. Innerhalb dieser Kontexte existieren spezifische soziokulturelle Konventionen, die ihrerseits wiederum Einfluss darauf nehmen, welche Identitäts- bzw. Rollenslots für die Akteure zu Verfügung stehen und wie die Übernahme dieser Slots (z. B. sprachlich) kodiert, d. h. symbolisch kommuniziert werden kann. Sie bilden damit die kulturellen Interpretationsrahmen, innerhalb derer beispielsweise Autorenidentitäten konstruiert werden können.

\footnotetext{
${ }^{10}$ In Face-to-face-Interaktionen spielen natürlich noch zusätzliche Faktoren in die Identitätskonstruktion hinein, wie z. B. Körperhaltung und -sprache, Stimme, Kleidung etc.
} 
Im Rahmen dieser Arbeit wird Kultur entsprechend als Code- oder Symbolsystem aufgefasst, das seinen Mitgliedern Bedeutungen und Interpretationsrahmen zur Verfügung stellt, an denen sie ihre Wahrnehmungen, aber auch ihre eigenen Handlungen ausrichten (zur Entwicklung eines symbolischen Kulturbegriffs s. z. B. Junge (2009); Moebius (2009); Reckwitz (2008), in Bezug auf Identität und Sprache s. Kresic (2006)) ${ }^{11}$ Hier relevante Symbole in diesem Sinne wären also die mehr oder weniger starke Personalisierung wissenschaftlicher Texte, der Gebrauch oder Nicht-Gebrauch von Personalpronomen und die Kombination dieser Pronomen mit bestimmten versprachlichten (textexternen) Handlungen. Diese Symbole können dann von anderen Mitgliedern der Gemeinschaft auf Basis des gemeinsamen kulturellen Verständnisses z. B. als „wissenschaftlich“ oder „nicht wissenschaftlich“, „studentisch“ etc. interpretiert werden (vgl. dazu die entsprechenden Leserurteile in den Untersuchungen von Hyland (2001b) und Steinhoff (2007)).

Hier werden insbesondere die versprachlichten Handlungen, die sich der Autor über das Pronomen der ersten Person zuschreibt, als relevante Symbole betrachtet. Aus der Menge aller möglichen Handlungen, die im Rahmen einer wissenschaftlichen Arbeit anfallen und die der Autor sich selber zuschreiben könnte, wird in den untersuchten Texten nur eine sehr kleine Auswahl tatsächlich mit dem ICH bzw. dem WIR als Subjekt und Agens realisiert. Dies sind, so meine Schlussfolgerung, die wissenschaftskulturell angemessenen Handlungen, über die die Autoren ihre Identität als Wissenschaftler kommunizieren.

Kultur wird hier sowohl als eine individuelle Eigenschaft der Mitglieder einer bestimmten Gemeinschaft als auch als diese Gemeinschaft selber gefasst ${ }^{12}$. Die kulturelle Gruppe stellt die Symbole, Symbolsysteme und Codes bereit und um erfolgreich verstanden zu werden und interagieren zu können, müssen die individuellen Mitglieder dieser Kultur diese Codes verwenden. Dies setzt voraus, dass die Individuen diese Codes gelernt bzw., z. B. beim wissenschaftlichen Schreiben in einer Fremdsprache, umgelernt haben. Dabei kommen in jedem Menschen viele verschiedene Codesysteme aus verschiedenen Gruppen zusammen und jeder Mensch hat in jeder Situation zumindest theoretisch die Möglichkeit auszuwählen, welches Codesystem er für seine

\footnotetext{
${ }^{11}$ Durch diese Definition von Kultur als Symbolsystem wird auch die häufig getroffene Unterscheidung zwischen Kultur und Domäne bzw. Kulturen und Subkulturen unnötig, da Domänen und Subkulturen als Symbolsysteme für sich kulturellen Charakter haben. Wichtiger ist es m. E., die jeweiligen Faktoren und Parameter zu spezifizieren, die in der Betrachtung von Bedeutung sind, und diese miteinander in Relation zu setzen. Dadurch wird auch berücksichtigt, dass man insbesondere in einer empirischen Arbeit nie alle kulturellen Faktoren, die Einfluss auf den Untersuchungsgegenstand haben, berücksichtigen kann. Dazu ist die Interpretation von kulturellen Codes zu sehr auch von den individuellen und situativen Kontexten abhängig. Durch den bewussten Fokus auf einzelne Parameter und die Reflexion der Tatsache, dass andere kulturelle Parameter gezielt ausgeklammert werden, wird man der Komplexität eines solchermaßen umfassenden Begriffs von Kultur gerecht, ohne der Gefahr eines unangemessenen Essentialismus zu unterliegen.

${ }^{12}$ Zum Begriff Kollektive Identität und zum Verhältnis kollektiver und individueller Identitäten s. Emcke (2000).
} 
Handlungen zugrunde legt (vgl. z. B. Hyland 2002a 1094). Dennoch kann man trotz aller Individualität, die auch in den für diese Arbeit untersuchten Texten beobachtbar ist, davon ausgehen, dass die Autoren bestimmte Codesysteme bevorzugt eingesetzt haben und dass sie entsprechend alle auch eine gemeinsame Schnittmenge von mindestens einer Gruppe haben, der sie angehören. Diese gemeinsame Schnittmenge wird hier als die relevante Ausprägung von Wissenschaftskultur aufgefasst. Dabei besteht eine ständige Wechselwirkung zwischen den Individuen einer Gruppe und dem gemeinschaftlichen Symbolrepertoire, durch das diese Gruppe kommuniziert. Kultur ist also, genau wie Identität "something that we actively and publicly accomplish through discourse, and so oriented to community as much as individuality" (Hyland 2009: 25).

Identity most often refers to human beings, often individuals, but also groups of individuals. When used in combination, culture and identity indicate some kind of interplay between their meanings. This implies that the identity of a person or group will be influenced or even shaped by culture while at the same time the person/group will influence and shape that culture. The development of culture is hence a two-way process with individuals interacting in forming and being formed. (Fløttum u. a. 2006. S. 16)

Der hier verwendete Kulturbegriff ist sehr weit gefasst und eignet sich entsprechend nicht gut für eine empirisch umsetzbare Operationalisierung. Aus diesem Grund müssen konkrete, operationalisierbare Faktoren bestimmt werden, die empirisch überprüfund untersuchbar sind. Dabei ist zunächst zu berücksichtigen, dass Kultur, genau wie Identität und Sprache, als Zoombegriff im Sinne Fritz Hermanns' betrachtet werden kann: „Damit ist gemeint, daß man den Skopus des Begriffs beliebig weit bzw. eng einstellen kann" (Hermanns 2012: 269). Daher soll hier anhand der für diese Arbeit als relevant erachteten Parameter die "Zoomeinstellung" diskutiert werden. Diese Parameter beeinflussen das wissenschaftliche Schreiben maßgeblich und haben aus diesem Grund auch die Auswahl der Korpustexte determiniert. Die Faktoren, die hier neben der allgemeinen Domäne Wissenschaft besonders berücksichtigt werden, sind der national-gesellschaftliche Kontext, die Sprache und die Fachdisziplin. Diese wirken konstitutiv auf das wissenschaftliche Schreiben, insbesondere auf die Ausprägung unterschiedlicher wissenschaftlicher Textsorten bzw. Genres (vgl. z. B. Swales 1990). Diese Faktoren bzw. Kontexte wurden auch in anderen Untersuchungen als die für den Gebrauch von Verfasserreferenzen und die Konstruktion von Autorenidentitäten relevanten zugrunde gelegt (vgl. z. B. Fløttum u. a. 2006; Koutsantoni 2007; Sanderson 2008a).

Four settings which may have an impact on the identity of the academic author are mentioned: the national/native language culture the author belongs to, leading to a national/language identity, the world of academia, 
providing the author with a general academic identity, the author's chosen discipline and a disciplinary identity, and finally the genre and the discourse community settings. (Fløttum u.a. 2006: 2)

Der national-gesellschaftliche Kontext wirkt dabei beispielsweise auf verschiedenen Ebenen auf das wissenschaftliche Schreiben ein. Zunächst bestimmt er das Bildungssystem und die schulischen Curricula, z. B. auch in Bezug auf die Schreibdidaktik 13 , Weiterhin wird hier der gesellschaftliche Stellenwert von Wissenschaft im Allgemeinen bestimmt. Auch das Ansehen von kollektiven gegenüber individuellen Forschungsleistungen wird zumindest teilweise im Rahmen von nationaler Forschungsförderung gelenkt. Die Sprache determiniert beispielsweise die Mittel zur möglichen (De-)Personalisierung (z. B. Passiv und Passiversatzformen, die Bedeutung unpersönlicher Pronomen etc.), aber auch, was auf sozio-pragmatischer Ebene in das Register der Wissenschaftssprache fällt. Und nicht zuletzt stellt sie mit den verschiedenen Textsorten oder Genres die möglichen Diskursformen (i. S. von Swales 1990: 6).

Bezüglich der Fachdisziplin lag der Fokus in vorhergehenden Untersuchungen vor allem auf den großen Fachbereichen der Natur-, Geistes- und Sozialwissenschaften, die anhand einzelner Teilfächer untersucht wurden (z. B. Sanderson 2008a: Steinhoff 2007; Fløttum u.a. 2006). Hier haben sich teilweise auffallende Unterschiede ergeben, insbesondere beim Vergleich der sog. hard und soft disciplines (z. B. Hyland 2000, 2002a). Die o.g. Untersuchungen haben aber auch gezeigt, dass es innerhalb dieser großen fachlichen Zusammenhänge gravierende Unterschiede zwischen den Teilfächern gibt, die sich aus unterschiedlichen Forschungsmethodiken oder verschiedenen übergeordneten Epistemologien ergeben können. Auch der Forschungsansatz (empirisch oder hermeneutisch) kann schon innerhalb einer Teildisziplin wie z. B. der Soziologie deutliche Unterschiede in der Ausgestaltung eines wissenschaftlichen Artikels hervorbringen. Hier wird noch einmal deutlich, wie wichtig es ist, einen geeigneten "Zoomfaktor" für eine Untersuchung dieser Art zu finden.

In dieser Arbeit liegt der Zoom auf einem einzelnen Fach der Sozialwissenschaften, der Soziologie, da dies insbesondere für die Datenerhebung eine vorteilhafte Perspektive war. Der Blick auf die höhere Ebene der Sozialwissenschaften wäre zu unscharf, da die zu vermutenden Unterschiede in der Schreibweise zwischen den sozialwissenschaftlichen Fächern ein deutlich größeres Korpus erfordert hätten, um aussagekräftige Ergebnisse zu erzielen. Ein engerer Fokus auf eine bestimmte Untergruppe soziologischer Autoren, z. B. Soziologen, die an dem gleichen Institut studiert haben, oder auch eine thematisch-methodologische Einschränkung der Texte, wäre ebenfalls deutlich aufwändiger und damit praktisch nicht umsetzbar gewesen. Entsprechend sind die Korpustexte in dieser Untersuchung an den Faktoren national-gesellschaftliche

\footnotetext{
${ }^{13} \mathrm{Zu}$ Unterschieden in der deutschen und US-amerikanischen Schreibdidaktik in Schule und Hochschule s. z. B. Foster (2006)
} 
Kultur (deutsch/US-amerikanisch), Sprache (Deutsch/US-Englisch) und (Teil)Disziplin (Soziologie) ausgerichtet.

Das oben beschriebene weite Kulturkonzept wird in Hinblick auf eine diskursiv und von (schriftlichen) Texten geprägte Gemeinschaft wie der wissenschaftlichen mit dem Begriff der Diskursgemeinschaft deutlich greifbarer. Die Diskursgemeinschaft bzw. discourse community stellt die soziokulturellen Rahmenbedingungen, denen die Entstehung von Texten unterliegt, in den Mittelpunkt (Swales 1990; Pogner 1997, 1999, 2003). Pogner (1999. 146) versteht dabei unter Diskursgemeinschaften „soziale Gruppen, die sich vor allem durch ihre spezifische Diskursform voneinander unterscheiden lassen“ und die sich „durch unterschiedliche, spezifische Muster des Sprachgebrauchs (und des Denkens) bei der sozialen Konstruktion und Aushandlung von Bedeutung aus[zeichnen]“. Damit stellt die Diskursgemeinschaft das sprachliche bzw. diskursive Symbolsystem bereit, anhand dessen ihre Mitglieder kommunizieren. Dieses Symbolsystem beschränkt sich allerdings nicht nur auf die sprachliche und formale Ausprägung der Texte bzw. Textsorten, die die Mitglieder der Diskursgemeinschaft benutzen, sondern umfasst bzw. beeinflusst auch, welche Inhalte in diesen Texten kommuniziert werden.

Mitglieder von Diskursgemeinschaften haben demnach nicht nur gemeinsame Annahmen darüber, welche Objekte/Themen als untersuchens- und diskussionswert gelten, mit welchen Methoden man/frau diese Objekte/ Themen untersucht und was Beweiskraft hat. Sie haben auch gemeinsame Vorstellungen davon, welche formalen Konventionen es bei der Präsentation der Untersuchungsergebnisse einzuhalten gilt. Deshalb beziehen sich ihre Konventionen sowohl auf die Inhalte als auch auf die Form der Textproduktion und -produkte. (Pogner 1999: 146)

Um discourse communities von anderen Gemeinschaften abgrenzen zu können bzw. um festzustellen, ob bestimmte Personen in bestimmten interaktionalen Relationen Mitglieder einer Diskursgemeinschaft sind oder nicht, hat John Swales (vgl. 1990; 24-27) sechs Definitionsmerkmale herausgearbeitet, anhand derer er Diskursgemeinschaften identifiziert:

1. Einigkeit über gemeinsame Ziele, nicht nur über einen gemeinsamen Gegenstand

2. Mechanismen, die die Kommunikation der Mitglieder untereinander gewährleisten

3. Teilnahme der Mitglieder an der Kommunikation, Gebrauch dieser Mechanismen, um Informationen und Feedback zu erhalten und auszutauschen

4. Gebrauch eines oder mehrerer spezifischer Genres zur kommunikativen Verbreitung und zum Erreichen der gemeinsamen Ziele und damit zusammenhängende spezifische Erwartungen an die Diskursteilnehmer: Angemessenheit des The- 
mas, Form, Funktion und Position von bestimmten diskursiven Elementen (im Text)

5. spezifische Terminologie, um effiziente Kommunikation zwischen den Mitgliedern zu sichern

6. Zugang zur Community erfolgt nur über ein bestimmtes Level an inhaltlichem und diskursivem Wissen und die Mitglieder der Community entwickeln sich vom Novizen zum Experten. Das Bestehen der Community hängt weiterhin von einem ausgewogenen Verhältnis zwischen Experten und Novizen ab.

Besonders der letzte Punkt macht deutlich, dass die Zugehörigkeit zu einer Diskursgemeinschaft wie der Scientific Community im Allgemeinen oder eines bestimmten Faches eines langwierigen Sozialisationsprozesses bedarf. Der angemessene Gebrauch der community-spezifischen Textsorten, die angemessene Darstellung von Inhalten und nicht zuletzt die diskursive Übernahme von Rollen und Identitäten innerhalb dieser Textsorten muss von den Novizen erlernt werden, bevor sie als vollwertige Mitglieder am Diskurs teilnehmen können.

Academic language socialization is the process by which individuals learn to enter into the discussions and again (sic!) access to the resources of academic disciplines through learning specialized language use and participating in academic activity settings. Learning to read and write in academic settings occurs through extended experiences in those settings, by meeting the expectations of those situations, and gaining from the opportunities for participation they offer. (Bazerman u. a. 2005: 8)

Dieser Sozialisationsprozess, der genaugenommen nie abgeschlossen ist, da Diskursgemeinschaften natürlich auch einem Wandel unterliegen (vgl. Sanderson|2008a: 69), muss in mehr oder weniger umfassenden Ausmaß jedes Mal neu durchlaufen werden, wenn eine Person in eine neue Gemeinschaft eintritt. Dies ist beispielsweise dann der Fall, wenn sich einer der oben beschriebenen kulturellen Parameter ändert, wenn ein Autor also in einer nationalkulturell, fachkulturell oder sprachlich anders geprägten Community Fuß fassen will.

\subsection{Zusammenfassung}

In diesem Kapitel wurden die theoretischen Grundpositionen dieser Arbeit erläutert. Wissenschaftliches Schreiben wird als soziale Interaktion verstanden, die praktisch immer in einem interkulturellen Raum stattfindet. Dies wird umso deutlicher, wenn man die relevanten Kulturen nicht nur als Nationalkulturen sieht, sondern Kultur als Symbolsystem versteht und dabei berücksichtigt, dass auch zwischen den einzelnen Fachbereichen und Disziplinen fachkulturelle Unterschiede bestehen. 
Im Rahmen des wissenschaftlichen Schreibens als sozialer Interaktion ist es notwendig, dass die Schreibenden sich eine soziale Identität als wissenschaftliche Autoren konstruieren. Diese muss den soziokulturellen Erwartungen der jeweiligen Diskursgemeinschaft entsprechen. Die Konstruktion dieser Identität erfolgt insbesondere über die Übernahme bestimmter, wissenschaftskulturell angemessener sozialer Rollen. Dies wiederum wird innerhalb eines wissenschaftlichen Artikels u. a. über Handlungen, die in der ersten Person vollzogen werden, kommuniziert.

In diesem Kapitel wurde entsprechend der dieser Arbeit zugrundeliegende, auf postmodernen und konstruktivistischen Ansätzen beruhende Identitätsbegriff definiert. Die Identität Wissenschaftlicher Autor ist nur eine von vielen verschiedenen Identitätsfacetten, die einer Person theoretisch zur Verfügung stehen und die sie, abhängig von der sozialen Situation sowie ihren eigenen Zielen und Vorlieben, einnehmen kann. Dabei wird Identität immer aktiv über den jeweiligen Diskurs konstruiert, wobei Sprache gerade in schriftlichen und asynchronen Kontexten eine entscheidende Rolle spielt.

Auch im wissenschaftlichen Schreiben haben Autoren die Möglichkeit, ihre Identität zu konstruieren, indem sie verschiedene soziale Rollen einnehmen. Soziale Rollen werden hier verstanden als Verhaltensmuster bzw. Handlungsschemata, die in einem bestimmten gesellschaftlichen Zusammenhang von den Mitgliedern dieser Gesellschaft erwartet werden und die sich im wissenschaftlichen Schreiben vor allem an den sprachlich dargestellten Handlungen ablesen lassen, die sich die Autoren über die erste Person zuschreiben.

Die Selbstdarstellung als Mittel der Identitätskonstruktion in wissenschaftlichen Artikeln wird im folgenden Kapitel tiefergehend dargestellt. Dazu wird die Textsorte Wissenschaftlicher Artikel beschrieben und es werden wichtige theoretische Ansätze zur Selbstdarstellung und zur sozialen Interaktion in wissenschaftlichen Artikeln vorgestellt. Weiterhin werden grundlegende Arbeiten zum Gebrauch der ersten Person Singular und Plural sowie des unpersönlichen MAN in deutsch- und englischsprachigen wissenschaftlichen Artikeln diskutiert. Darauf basierend wird der rollentheoretische Ansatz dieser Arbeit herausgearbeitet. 


\section{Kapitel 3}

\section{Selbstdarstellung in wissenschaftlichen Artikeln}

\subsection{Die Textsorte „,Wissenschaftlicher Artikel“}

Der wissenschaftliche Artikel, insbesondere der Forschungsartike $]^{1}$, gilt inzwischen als wichtigste Textsorte in der wissenschaftlichen Kommunikation überhaupt und wird auch in den Geistes- und Sozialwissenschaften, wo lange Zeit die Monographie als die relevanteste Veröffentlichungsform galt (Auer und Baßler 2007b: 25-26), immer wichtiger (vgl. Graefen und Thielmann 2007: 67).

Der wissenschaftliche Artikel ist aufgrund seiner Kürze, seiner relativ kurzen Produktionszeit und seiner großen Reichweite besonders gut geeignet, einen Wissenschaftler im sozialen Zusammenhang seiner Disziplin zu verorten, wobei hier diese Verortung auch, aber nicht nur inhaltlich zu verstehen ist. Insofern spielt er insbesondere für die Konstruktion einer angemessenen Autorenidentität auch im Rahmen einer generellen Positionierung in der wissenschaftlichen Gemeinschaft eine bedeutende Rolle. Er dient damit nicht nur der Vermittlung und Verbreitung neuen Wissens, sondern hilft dem Verfasser auch und grade dabei, Reputation herzustellen, sich einen Namen zu machen und sich in der Gemeinschaft zu verorten bzw. sich überhaupt erst in die Gemeinschaft einzubringen. Als wichtigster (kommunikativer) Zweck eines wissenschaftlichen Artikels wird allerdings trotzdem häufig die Produktion und Verbreitung neuen Wissens gesehen.

\footnotetext{
${ }^{1}$ Mit Forschungsartikel im engeren Sinne ist hier ein Artikel gemeint, der die Ergebnisse einer (im hier untersuchten Korpus empirischen) Forschung darstellt, im Gegensatz zu theoretisch orientierten Artikeln. Da das hier untersuchte Korpus auch Texte enthält, die sich nicht direkt oder in erster Linie auf Forschungsergebnisse stützen, sondern eher theoretisch gehalten sind, und die Benennung Forschungsartikel zumindest im engeren Sinne damit irreführend wäre, habe ich mich für die etwas weiter gefasste Bezeichnung wissenschaftlicher Artikel entschieden. In anderen Untersuchungen ist synonym von Abhandlungen (Kussmaul 1978), Aufsätzen (Kresta 1995), Fachzeitschriftenartikeln (Hutz1997), Artikeln (Steinhoff 2007), research oder gelegentlich auch academic articles (Sanderson 2008a: Fløttum u. a. 2006; Hyland 2000) die Rede.
} 
Natürlich ist die Produktion von Wissen für den einzelnen Forscher nicht der einzige Antriebsfaktor zur Veröffentlichung von Texten. Forscher sind zudem bestrebt, durch ihre Texte in der scientific community Reputation zu erlangen. Das Streben nach Reputation ist jedoch der Erkenntnisproduktion erkennbar nachgeordnet: Ohne einen signifikanten Beitrag zum wissenschaftlichen Wissen erfährt kein Wissenschaftler die erwünschte Anerkennung. (Steinhoff 2007; 110-111)

Diese Sichtweise ist jedoch in dieser umfassenden Form fraglich. Natürlich spielt die Verbreitung von Wissen immer eine wichtige Rolle und selbst in mündlichen Vorträgen ist die Selbstdarstellung des Redners normalerweise nicht der zentrale Fokus des Publikums. Dennoch ist es für einen Wissenschaftler sicherlich ebenso wichtig, Artikel zu veröffentlichen, um Reputation zu erhalten, denn das bedeutet, als Mitglied der Community akzeptiert zu werden. Dies betrifft nicht nur die Akzeptanz der anderen Wissenschaftler der Community, sondern auch und besonders den Zugang zu materiellen Mitteln über die Vergabe von Forschungsgeldern, die Berufung auf Professuren usw. Darüber hinaus gelten Veröffentlichungen, insbesondere in anerkannten Organen des jeweiligen Faches, ebenfalls als wichtige Indikatoren für erfolgreiche wissenschaftliche Aktivität (Weingart 2003: 32).

Nicht selten überlagert die Funktion der Positionierung diejenige der Wissensvermittlung sogar: Wenn man verschiedene Artikel eines Autors zu gleichen oder ähnlichen Themen betrachtet, stellt sich häufig heraus, dass ein Mehr an Veröffentlichungen für den Autor nicht unbedingt immer ein Mehr an Erkenntnisgewinn für die Gemeinschaft bedeuten muss. Die Publikation ähnlicher Inhalte in unterschiedlichen Organen ermöglicht ggf. nicht nur anderen Personenkreisen den Zugang zum hier verbreiteten Wissen, sondern macht den jeweiligen Autor auch in diesen fachlichen Gemeinschaften bekannt und eröffnet so ggf. weitere Möglichkeiten der Ressourcengewinnung. Und auch das Bestreben, durch die Veröffentlichung inhaltlich ähnlicher Artikel in unterschiedlichen Publikationen die eigene Publikationsliste zu erweitern, d.h. die aktuelle „Publish-or-Perish-Kultur" zu bedienen, ist schon lange nicht mehr nur auf die anglo-amerikanischen Länder beschränkt. „Wissenschaftliches Renommee“ werde in den Geisteswissenschaften (und sicherlich auch in den Sozialwissenschaften), so Auer und Baßler (2007b: 26), ,zunehmend durch die Anzahl der Veröffentlichungen gemessen".

Die besondere Relevanz von Glaubwürdigkeit im wissenschaftlichen Kontext, die Hyland beispielsweise direkt aus der Selbstdarstellung von Autoren in ihren Artikeln ableitet (z. B. Hyland 2002a, b, 2003, 2010), hebt auch Weingart hervor, indem er die Reputation von Wissenschaftlern mit ihrer Glaubwürdigkeit verbindet und diese in direkten Zusammenhang mit der ökonomischen Seite des Wissenschaftsbetriebes bringt: 
Reputation bedeutet in der Wissenschaft besondere Glaubwürdigkeit, und Glaubwürdigkeit verschafft besondere Aufmerksamkeit, die sich in die Zuweisung von Ressourcen übersetzen lässt. (Weingart|2003 23)

Die von Weingart genannte Glaubwürdigkeit, auf der die Reputation beruht, ist auch abhängig davon, wie man sich und seine Ergebnisse darstellt. Die Basis für eine glaubwürdige und damit erfolgreiche wissenschaftliche Arbeit liegt darin, dass man diese Arbeit und ihre Ergebnisse auf angemessene Weise kommuniziert und die anderen Mitglieder der Community davon überzeugt, dass das neue Wissen, das man ihnen anbietet, auf die im jeweiligen fachlichen Kontext als richtig erachtete Art erworben wurde. Dies geht nur, wenn man die soziokulturellen Kommunikationsweisen beherrscht und die üblichen Hürden auf dem Weg zur Veröffentlichung überwindet.

Papers that become published are writings that have managed to overcome the scrutiny of peer reviewers and referees, their multi-level evaluations and criticisms, and have ultimately gained legitimacy in the eyes of community gatekeepers. (Koutsantoni|2007; 32)

Diese Legitimität erhält der Autor dadurch, dass er in seinem Artikel eine glaubwürdige Persona aufbaut, mit der er von der Gemeinschaft als kompetentes Mitglied anerkannt werden kann. Dies ist die Basis für seine Argumentation, von der letztlich auch die Verbreitung des von ihm geschaffenen neuen Wissens abhängt (Hyland 2002b; Ivanič 1998). Ohne eine angemessen konstruierte wissenschaftliche Identität ist es nur schwer vorstellbar, glaubwürdig zu argumentieren und damit seine eigenen neuen Ergebnisse zu „vermarkten“, egal wie bahnbrechend diese auch sein mögen. Das oberste Ziel eines Artikels ist schließlich, die Leser von den präsentierten Inhalten und Schlussfolgerungen zu überzeugen. Dies geht aber nur, wenn der Autor sie zunächst rhetorisch davon überzeugen kann, dass er ein kompetentes und glaubwürdiges Mitglied der Community ist. Jemand, der nicht als Teil der Gemeinschaft anerkannt wird, weil er sich in seinem Artikel nicht überzeugend als Wissenschaftler bzw. wissenschaftlicher Autor darstellt, wird seine Ergebnisse nicht verbreiten können, schon weil die Wahrscheinlichkeit, dass der Artikel gedruckt wird, sehr niedrig ist (vgl. Graefen und Thielmann|2007; 69).

[T]o take part in the continuous dialogue which academic communication represents implies that the author must develop a persona willing to claim authority as a scholar. Only then can his or her research contribution be presented in a rhetorically convincing manner. (Fløttum u. a. 2006 19)

Und gerade in den Geistes- und Sozialwissenschaften, die vorrangig auf interpretative und hermeneutische Verfahren zurückgreifen, sind Ergebnisse, Analysen und Interpretationen niemals unabhängig von der Person zu sehen, die sie erzielt hat. Hier 
besteht also für die Autoren wissenschaftlicher Artikel eine besondere Notwendigkeit, eine authoritative, d. h. kompetente und glaubwürdige, Identität zu konstruieren, um die eigene Glaubwürdigkeit aufzubauen und zu untermauern.

\subsection{Theoretische Ansätze}

In der internationalen Forschung existiert eine Vielzahl von Studien zum Thema „Identität im wissenschaftlichen Schreiben“ (s. z. B. Koutsantoni 2007; Vassileva 2000), die auf unterschiedliche theoretische Ansätze zurückgreifen. Im Weiteren werden mit Voi$c e^{2}$ und Stance and Engagement zwei wichtige Ansätze aus der englischsprachigen Forschung vorgestellt, die eine eindeutig interaktionale Perspektive vertreten. Dabei kann Voice als die grundlegende Idee angesehen werden, zu der Hylands Modell von Stance and Engagement eine mögliche Operationalisierung darstellt (Hyland 2008).

\subsubsection{Voice}

Voice ist seit den 1960er Jahren eines der wichtigsten Konzepte der Schreibforschung und -didaktik im angloamerikanischen Raum. Es findet in allen Bereichen des Schreibens Verwendung (vgl. z. B. Bowden 1999, vii) und wird in der Analyse literarischer Texte ebenso benutzt wie in schulischen Curricula als Kriterium zur Bewertung schulischer Schreibaufgaben (Zhao und Llosa 2008, 154). Der Begriff Voice ist jedoch alles andere als unumstritten oder gar klar definiert. Verschiedene Autoren verstehen darunter teilweise sehr unterschiedliche Dinge (vgl. z. B. Elbow 1994: Bowden|1995). Grundsätzlich kann man jedoch sagen, dass Voice im allgemeinen die persönliche Stimme des Autors darstellt und entsprechend dort auftritt, wo Autoren ihre eigene Meinung und persönliche Einstellungen äußern, ihre eigene Person (im weitesten Sinne) beschreiben oder ihre Kompetenz und Zuverlässigkeit (authoritativeness) demonstrieren (vgl. Hyland 2008: 5).

The concept of 'voice' helps us to understand how writers typically position themselves and their work by claiming solidarity with readers, evaluating ideas and acknowledging alternative views, so that controlling their disciplinary voice, or level of authorized personality in a text, becomes central to building a convincing discourse. This view sees academics as not only producing texts that plausibly represent an external reality, but using langua-

\footnotetext{
${ }^{2}$ Das Konzept Voice spielt nicht nur in der Sprachwissenschaft eine Rolle, sondern wird auch in der Literaturwissenschaft verwendet. Aus Platzgründen kann dieser Bereich hier jedoch nicht weiter beleuchtet werden, auch wenn der Vergleich von wissenschaftlichem Schreiben und literarischem Schreiben insbesondere unter dem Aspekt der Selbstdarstellung des Autors sicherlich weitere interessante Ansatzpunkte bringen würde. Für weitere Informationen zum Gebrauch dieser Konzepte außerhalb der Sprachwissenschaft sei auf die in den jeweiligen Abschnitten genannte Einführungs- und Überblicksliteratur verwiesen.
} 
ge to acknowledge, construct and negotiate social relations. Put succinctly, every successful academic text displays the writer's awareness of both its readers and its consequences. (Hyland|2008, 6)

Voice betrifft also sowohl die Beziehung zwischen dem Autor und dem Inhalt seines Textes als auch zwischen dem Autor und seinen Lesern. Dabei liegt das Augenmerk in dieser Untersuchung darauf, wie der Autor diese Beziehung darstellt, nicht wie der Leser die Beziehung über den Text interpretiert (vgl. Bowden 1999: 35-36). Voice dient in diesem Zusammenhang dazu, den Autor eines Textes zu repräsentieren bzw. eine entsprechende Persona zu konstruieren und erfüllt damit eine notwendige soziale und kommunikative bzw. interaktionale Funktion:

Voice [...] is not an optional extra but an aspect of how we posititon ourselves in relation to our communities. (Hyland 2008: 6)

Die Art und Weise, wie Voice im wissenschaftlichen Schreiben realisiert wird, ist sehr stark kulturell bedingt (Hirvela und Belcher 2001; 85-86). Dies macht es insbesondere beim Schreiben in der L2 so schwer, eine angemessene Voice zu finden. Dabei scheint ein wesentliches Problem darin zu liegen, den Erwerb einer neuen (L2-)Voice mit bereits bestehenden Konzepten von Voice und der Darstellung der eigenen Identität und Persönlichkeit im wissenschaftlichen Schreiben in der L1 in Einklang zu bringen (Hirvela und Belcher 2001: 86-88). Dies gilt insbesondere dann, wenn diese sich in der L1 und der L2 stark voneinander unterscheiden. Hier ist es für Schreibdidaktiker sowie die betroffenen Autoren hilfreich, die Hintergründe dieser unterschiedlichen Umsetzungen von Voice zu kennen.

Therefore, we need to better understand the nature of these struggles [der Studierenden beim Finden ihrer Voice in der L2, J.S.] so as to more effectively serve the needs of our students and help them to find means of selfrepresentation in written English that complement rather than replace the identities they possess when they come to our writing classes. (Hirvela und Belcher|2001: 88)

Obwohl das Konzept Voice seit Jahrzehnten ein wesentlicher Bestandteil der internationalen Diskussion um Schreiben an sich und wissenschaftliches Schreiben im Besonderen ist? ist es in der deutschsprachigen Forschung zum wissenschaftlichen Schreiben und seiner Didaktik bisher so gut wie gar nicht berücksichtigt worden (vgl. z. B. die ansonsten umfassenden Arbeiten von Pohl|2007: Steinhoff 2007).

Aber auch in vergleichenden Untersuchungen zu deutschen und englischsprachigen Texten wird dieses Phänomen in der deutschen Forschung erstaunlicherweise nicht

\footnotetext{
${ }^{3}$ Für einen umfassenden Überblick über diese Diskussion s. z. B. Elbow (2007), einen Beitrag gegen Voice liefert (Bowden|1999)
} 
angesprochen, auch nicht in Untersuchungen, die sich mit den wesentlichen sprachlichen Merkmalen, über die Voice vermittelt wird (wie z. B. dem Gebrauch von Pronomen der ersten Person oder Hedging), auseinandersetzen (s. z. B. Kresta 1995; Hutz 1997). Eine wichtige Ausnahme stellt hier der Artikel von Katrin Girgensohn (Girgensohn 2008) dar, in dem sie genau dieses Phänomen auch für die Didaktik des wissenschaftlichen Schreibens thematisiert.

Nichtsdestoweniger wird auch Studierenden und Nachwuchswissenschaftlern in Deutschland als wichtiger Rat immer wieder mitgegeben, dass sie in ihren Haus-, Abschluss- und Qualifikationsarbeiten Themen behandeln sollen, die „ihnen liegen“, d.h. die zu ihnen passen, damit sie überzeugend schreiben können (z. B. Kornmeier 2008. 32). Dies soll dann letztlich zu dem führen, was Peter Elbow unter Writing with Power versteht und in seinem gleichnamigem Buch in den Kapiteln zu Voice behandelt:

[...] power comes from the words somehow fitting the writer (not necessarily the reader). That good fit between the writer and her words makes for resonance: the words bore through to readers no matter what their disposition. (Elbow 1998: 280)

Auch wenn das Konzept Voice in der deutschsprachigen Forschung keine vergleichbare Rolle spielt wie in der angloamerikanischen, kann man also davon ausgehen, dass das Phänomen selber durchaus vorhanden ist. Auch in deutschen wissenschaftlichen Texten ist aus interaktionaler Perspektive immer die Frage zu stellen, wie der jeweilige Autor in seinem Text seine eigene wissenschaftliche Persona konstruiert und sich einerseits zu seinem Gegenstand und andererseits zu seinen Lesern bzw. der wissenschaftlichen Gemeinschaft im Allgemeinen in Beziehung setzt. Darüber hinaus ist auch zu bedenken, dass Voice ein wesentlicher Bestandteil der akademischen Schreibausbildung in den USA ist. Dies bedeutet letztlich, dass, auch wenn es in der deutschen akademischen Ausbildung nicht explizit thematisiert wird, es dennoch als wichtiges Bewertungskriterium für "gutes" wissenschaftliches Schreiben im angloamerikanischen Raum gilt und damit spätestens dann für deutsche Wissenschaftler relevant wird, wenn sie dort ihre Artikel veröffentlichen wollen.

\subsubsection{Stance and engagement}

Basierend auf dem Voice-Konzept und v. a. der Annahme, dass ein wissenschaftlicher Autor immer auch sein Publikum vor Augen haben muss, um einen erfolgreichen wissenschaftlichen Text zu schreiben, hat Ken Hyland sein Modell von Stance and Engagement im wissenschaftlichen Schreiben entwickelt (s. im Folgenden v. a. Hyland 2001a, 2005, 2008). Damit untersucht er die sprachlichen Mittel, anhand derer wissenschaftliche Autoren die Interaktion mit ihrer Fachcommunity (auf Englisch) konstruieren (Hyland 2005: 173). 
Um eine soziale Beziehung zu seinen Lesern aufzubauen, hat ein wissenschaftlicher Autor verschiedene, sich teilweise überschneidende, (Cluster von) sprachlichen Mitteln zur Verfügung. Wissenschaftliche Autoren müssen dabei mit zwei Interaktionsebenen umgehen (vgl. Hyland|2005: 174):

1 Der Autor präsentiert sich selbst. (Stance)

2 Der Autor bezieht Leser mit ein. (Engagement)

Unter Stance versteht Hyland dabei die sprachliche Darstellung der eigenen Voice des Autors, also die Kommunikation der eigenen Einstellungen, Meinungen und Überzeugungen. Dabei hebt er die drei Hauptkomponenten evidentiality, affect und presence hervor (Hyland 2005: 178). Wissenschaftliche Autoren drücken Stance also vorrangig aus, um ihre eigene Sorgfalt und Präzision hervorzuheben und dadurch ihre Glaubwürdigkeit zu unterstreichen. Daneben müssen sie dem Leser gegenüber Angaben machen, wie stark sie sich zu bestimmten Aussagen bekennen und diese unterstützen, wobei sie nicht zuletzt auch affektive und emotionale Mittel nutzen, um ihre persönlichen Einschätzungen und Bewertungen zu kommunizieren. Dabei haben sie immer die Wahl, wie persönlich oder unpersönlich sie ihren Text gestalten, wie sehr und auf welche Weise sie sich in ihren Text einbringen und sich sichtbar machen (Hyland|2005: 178).

Engagement repräsentiert dagegen stärker die dialogische bzw. interaktionale Perspektive und beschreibt, wie ein Autor eine Beziehung zu seinen (angenommenen) Lesern aufbaut, beispielsweise indem er sie in seine Argumentation mit einbezieht, ihre Aufmerksamkeit lenkt und möglichen Gegenargumenten begegnet und sie so Schritt für Schritt zu seinen Ergebnissen oder zu bestimmten Schlussfolgerungen führt ( $\mathrm{Hy}-$ land 2005: 176). Dies geschieht beispielsweise dadurch, dass er seine Leser an argumentativ kritischen Stellen durch den Gebrauch rhetorischer Fragen, Direktiva und den Bezug zu als allgemein verstandenem Fachwissen direkt einbindet (Hyland 2005: 182).

Um einen überzeugenden und erfolgreichen Text zu schreiben, muss der Autor also ein möglichst genaues Bild von seinen Lesern haben, er muss mögliche Einwände gegen seine Argumente vorhersehen und angemessen darauf reagieren (Hyland 2005 182). Dadurch, dass er dabei den fachspezifischen Anforderungen folgt, demonstriert er seine Zugehörigkeit zu und Solidarität mit der Fachcommunity.

Stance und Engagement bilden also gemeinsam eine wesentliche Dimension evaluativer und persuasiver Texte wie z. B. wissenschaftlicher Artikel. Um diese beiden Dimensionen der Interaktion sprachlich auszudrücken, stehen dem Autor verschiedene rhetorische Mittel zur Verfügung. Hyland hat basierend auf der Untersuchung seines Korpus aus 240 Forschungsartikeln aus acht verschiedenen Fächern sowie Expertenmeinungen von Wissenschaftlern, die in diesen Fächern verortet sind und ihre eigenen Schreibgewohnheiten in Interviews reflektiert haben, herausgearbeitet, dass Stance in wissenschaftlichen Texten vorrangig über Hedges und Boosters, Attitude markers und 
Self-mention realisiert wird. Engagement drücken wissenschaftliche Autoren vorrangig anhand von Reader pronouns, Directives und Questions sowie über die sprachliche Konstruktion von Shared knowledge und Personal asides aus.

Hyland fasst sein Konzept von Stance und Engagement in der folgenden Darstellung zusammen $4^{4}$

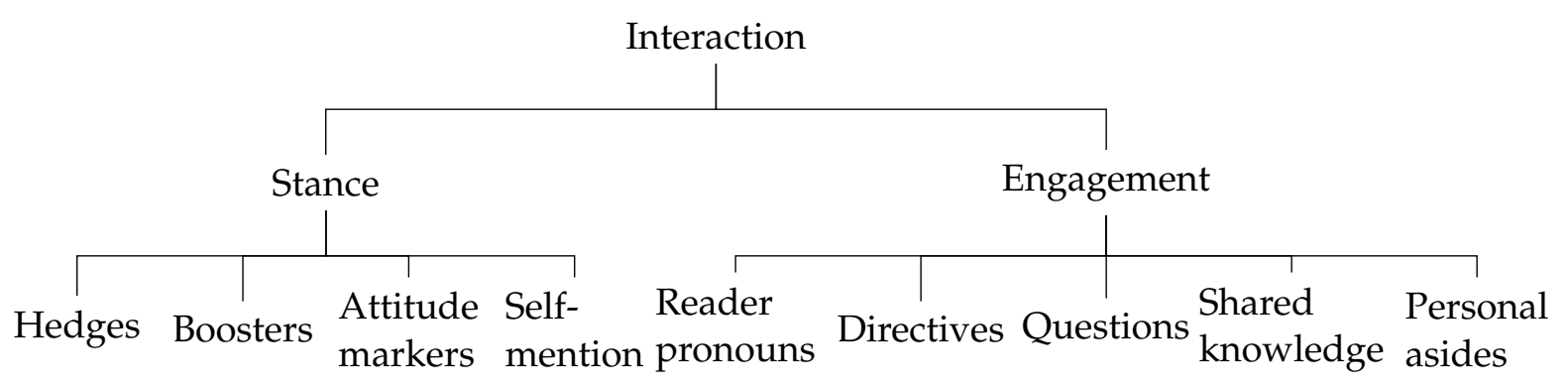

Abbildung 3.1: Sprachliche Mittel der Interaktion in wissenschaftlichen Texten aus $\mathrm{Hy}-$ land (2005: 177)

Da die Realisierung von Stance und Engagement ein so wichtiges Element wissenschaftlicher Artikel ist, ist die Beherrschung der ihr zugrundeliegenden soziokulturellen und linguistischen Regeln auch mit ausschlaggebend, um einen adäquaten Text zu produzieren. Dabei erfüllen gerade die hier untersuchten Pronomen als wichtige Mittel der Selbstbenennung (Self-mention) in dieser Hinsicht eine wesentliche Funktion (Hyland 2005: 181).

\subsection{Sprachliche Mittel der Selbstdarstellung}

Zur sprachlichen Darstellung des eigenen wissenschaftlichen Selbst stehen sowohl im Englischen als auch im Deutschen eine Reihe verschiedener Mittel zur Verfügung. Insbesondere wenn man eine weitgefasste Vorstellung dieses Selbst hat, die auch die Kommunikation von Meinungen und Einstellungen umfasst, wird deutlich, dass es viele verschiedene Möglichkeiten gibt, dies sprachlich umzusetzen.

Neben dem Gebrauch von Personalpronomen der ersten Person und des unpersönlichen MAN, die in dieser Arbeit betrachtet werden, wurden bisher vor allem der Einsatz von Heckenausdrücken bzw. Hedges (z. B. Hyland 1998; Graefen 2000), Selbstzitation (z. B. Aksnes 2003; Hyland 2003) und Ablativ-Subjekte wie „der Autor/the author" (z. B. in Kresta 1995) untersucht. Maurizio Gotti (Gotti 2009b; 10) nennt als mögliche Mittel zur Markierung von Autorenidentität neben den hier untersuchten Pronomen auch die Realisierungsformen epistemischer Modalität, argumentative Konnektoren, negative und konzessive Strukturen sowie bestimmte Lexeme und metatextuelle Ausdrücke. Steinhoff (Steinhoff 2007, 165-166) hat mit Perspektive auf das Deutsche

\footnotetext{
${ }^{4}$ Zur näheren Erklärung der sprachlichen Mittel, die zur Umsetzung von Stance und Engagement zur Verfügung stehen, s. z. B. Hyland (2005, 2008)
} 
„Mittel, mit denen Verfasser wissenschaftlicher Texte auf sich selbst verweisen, wie sie solche Verweise ,kodieren“" identifiziert und stellt die folgende Liste auf:

- Zustandspassiv: „sei die Anmerkung erlaubt“

- „werden“-Passiv \& Modalverb: „kann/muss/soll/darf die Frage gestellt werden"

- „lassen“\& „sich“ \& V (Inf.): „lässt sich sagen“

- „,sein“ \& ,zu“ \& V (Inf.): ,ist zu diskutieren“

- „,ein" \& Adjektiv mit Sufix ,-bar" und „-lich“: „ist erkennbar" , „ist vermutlich“

- Partizipialkonstruktionen: „der zu behandelnde Aspekt“

- Funktionsverbgefüge: „findet im nächsten Kapitel Berücksichtigung“

- Nominalisierungen: „durch die Darstellung der Thematik“

- Bescheidenheitsverben: „scheint tragfähiger zu sein“

- Subjektschübe: „nimmt dieses Kapitel Bezug auf“

- „wie“-Sätze: „wie erläutert worden ist"

- Lexem „Verfasser(in)“ o. Ä.: "geht der Verfasser von ... aus"

- Meinungsausdrücke: „ist meines Erachtens“ (Steinhoff 2007; 165-166)

Bevor hier jedoch der Fokus voll auf die Pronomen der ersten Person gerichtet wird, die sicherlich die deutlichsten Mittel sind, mit denen wissenschaftliche Autoren sich und ihre Persona explizit selber benennen und damit in den Diskurs einbringen, soll hier kurz der eigentliche Default-Case im wissenschaftlichen Schreiben diskutiert werden, nämlich die bewusst unpersönliche Darstellungsweise (vgl. Koutsantoni 2007 151).

Der Gebrauch unpersönlicher Wendungen im wissenschaftlichen Schreiben wird vorrangig als Mittel zur Objektivierung von Forschung und Ergebnissen betrachtet. Die Person des Wissenschaftlers wird so weit wie möglich in den Hintergrund gestellt, wodurch der Eindruck erzeugt wird, die Ergebnisse und Fakten sprächen für sich und die wissenschaftliche Arbeit beruhe auf objektivierbaren Daten und Methoden, die unter gleichen physischen Gegebenheiten zwangsläufig zu den gleichen Ergebnissen führen werden. Besonders auffällig ist in diesem Zusammenhang der häufige Verzicht auf die Selbstbenennung des Autors anhand der Personalpronomen der ersten Person, der von Weinrich (1989) als "Ich-Tabu“ (s. auch Kretzenbacher und Weinrich 1995: 33-34) bezeichnet wird. Diese Strategie soll dazu beitragen, die Objektivität der wissenschaftlichen Forschung zu unterstreichen und die zu vermittelnden Informationen als personenunabhängig darzustellen. Die Subjektivität des Wissenschaftlers und ihr Einfluss auf den Forschungsprozess wird so bewusst heruntergespielt und das Infragestellen der Ergebnisse möglichst unterdrückt, um die eigene Person vor einem möglichen Gesichtsverlust zu schützen.

Attributing a claim to some impersonal agency and omitting to mention the author's personal involvement in its formulation are ways scientists use 
to distance themselves from their claims in an effort to protect themselves from possible negative criticism. (Koutsantoni 2007 155)

Wissenschaftliches Schreiben wird aus dieser Perspektive auch nicht, so wie andere Schreibprozesse, als kreativer Prozess betrachtet, sondern dient nur dazu, „natürliche Fakten " zu dokumentieren. Dies impliziert, dass es eine objektive Realität gibt, die von den subjektiven, individuellen Eigenschaften der Untersuchenden nicht beeinflusst wird. Diese Annahme gilt spätestens seit der epistemologischen Anschauungsweise des Konstruktivismus als fraglich. Insbesondere die Geistes- und Sozialwissenschaften arbeiten auch und gerade in ihren methodologischen Bereichen mit der konstruktivistischen Überzeugung, dass gerade die Subjektivität des Wissenschaftlers, die durch seine individuellen Interessen und Vorerfahrungen geprägt ist, ein wichtiger Auslöser für bestimmte Interpretationen, Analyseergebnisse und nicht zuletzt auch Fragestellungen ist.

Es ist nicht verwunderlich, dass dieser Wechsel in der epistemologischen Perspektive sich auch in den Diskurskonventionen der verschiedenen wissenschaftlichen Gemeinschaften widerspiegelt. In diesem Zusammenhang ist vor allem im englischsprachigen Raum aktuell die Entwicklung zu erkennen, dass wissenschaftliche Artikel in verschiedenen Fachdisziplinen einerseits immer mehr Informationsdichte aufweisen und andererseits immer subjektiver und persönlicher werden (Mair|2008). Dies erklärt auch, warum sich die Wissenschaftssprachlinguistik mit diesen Aspekten des wissenschaftlichen Schreibens seit Längerem auseinandersetzt. Im folgenden Teil werden daher die für die nachfolgende Untersuchung relevanten Studien zum Gebrauch von Verfasserrefenzen mit der ersten Person Singular und Plural sowie dem unpersönlichen MAN vorgestellt und verglichen. Dabei liegt speziell bei den deutschsprachigen Untersuchungen ein Fokus auf Analysen, die sich mit dem Vergleich des Deutschen und des Englischen auseinandersetzen.

\subsubsection{Personalpronomen}

Das Sprachenpaar Deutsch-Englisch ist im Allgemeinen eines, das sehr gut untersucht ist, insbesondere natürlich von deutscher Seite. Und auch in der Forschung zum wissenschaftlichen Schreiben nimmt dieses Sprachenpaar eine prominente Rolle im deutschsprachigen Diskurs ein. Dies liegt nicht zuletzt daran, dass Englisch weltweit betrachtet zurzeit als wichtigste Wissenschaftssprache gilt (z. B. Vassileva 2000: 32). Entsprechend gibt es eine Vielzahl an Arbeiten, die sich mit der Textsorte "Wissenschaftlicher Artikel“ beschäftigen und deutsch- und englischsprachige Artikel miteinander vergleichen (u. a. Clyne 1991: Graefen|1994: Trumpp 1998; Fandrych und Graefen 2002; Thielmann 2009). Dabei sind in den letzten Jahren diverse Aspekte in den Fokus der Untersuchungen gerückt, die in dieser Arbeit jedoch nicht alle berücksichtigt werden können. Hier liegt der Fokus auf ausgewählten Arbeiten, die sich konkret mit dem 
Gebrauch von Pronomen der ersten Person, also von Verfasserreferenzen, auseinander setzen. Es werden daher im Folgenden die Ergebnisse relevanter Arbeiten vorgestellt, die sich aus unterschiedlicher Perspektive mit dem Gebrauch von Personalpronomen im akademischen und wissenschaftlichen Schreiben beschäftigen.

Personalpronomen sind das deutlichste Mittel, um in der direkten Kommunikation auf sich selber (Sprecher, 1. Person) oder das Gegenüber (Hörer, 2. Person) zu verweisen. Dadurch können sich die jeweils Sprechenden zu ihren Hörern und auch der weiteren Umwelt in Beziehung setzen, d.h. sich positionieren. Und auch in geschriebener Kommunikation und insbesondere im wissenschaftlichen Artikel spielen Personalpronomen diese grundlegende Rolle.

We believe that the choice of a certain personal pronoun for a given context, or even the presence or non-presence of a personal pronoun in journal articles, and particularly in scientific journal articles, can often reveal how writers view themselves, their relationship with readers, and their relationship with the discourse community they belong to. (Kuo 1999. 123)

Die Personalpronomen dienen also vor allem zum Aufbau von Beziehungen zwischen dem Autor und der relevanten Umwelt, also dem Leser und der wissenschaftlichen Gemeinschaft, der Autor und Leser im Allgemeinen angehören. Das heißt, sie erfüllen in erster Linie eine soziale Funktion. Es bedeutet weiterhin, dass der Gebrauch von Personalpronomen, auch und gerade im wissenschaftlichen Schreiben, einen fundamental strategischen Wert hat (vgl. z. B. Fløttum u.a. 2006: 96). Die Pronomen werden als rhetorische Mittel benutzt, um die Ziele des Autors voranzutreiben, d. h. insbesondere, die Leser von seinen Argumenten zu überzeugen, indem sie dazu beitragen, eine angemessene und überzeugende wissenschaftliche Persona aufzubauen (z. B. Hyland 2011. 195).

Die Frage, die in dieser Untersuchung gestellt wird, ist also, wie die Pronomen der ersten Person in den Artikeln der beiden hier untersuchten Wissenschaftskulturen gebraucht werden und welche sozialen Rollen durch sie realisiert werden, mit denen sich die Autoren mit ihren Lesern und der gesamten Community in Beziehung setzen. Und auch, wenn hier ausschließlich Texte untersucht werden, die von erfahrenen Autoren in ihrer L1 verfasst wurden (vgl. Kap. 4), schwingt natürlich übergeordnet immer die Frage mit, ob und wie diese spezifischen Konventionen und die wissenschaftskulturellen Regeln, die ihrem Gebrauch zugrunde liegen, zu Problemen für Studierende oder auch für L2-Sprecher/Schreiber führen können und wie eine entsprechende Didaktik dazu beitragen kann, diese Probleme zu verringern (s. Kap.6).

\subsubsection{1. Person Singular}

Das Pronomen der ersten Person Singular erscheint auf den ersten Blick als verhältnismäßig einfach, gerade wenn man es mit der pragmatisch-diskursiven Komplexität der 
Pluralformen vergleicht (s. Kap. 3.3.1.2). Das ICH bezeichnet den Produzenten eines Textes, also den Sprecher oder, bei einem schriftlichen Text, den Autor. Dabei ist es, anders als das WIR, semantisch eindeutig. Es identifiziert den Textproduzenten in einem räumlichen und zeitlichen Kontext und funktioniert als Index für die reale Person, die den jeweiligen Text verfasst hat. Diese Person wird im wissenschaftlichen Artikel als Autor des Textes auch immer namentlich benannt, so dass eine eindeutige Zuordnung der Person möglich ist. $:^{5}$

Neben dieser spatio-temporalen Indizierung sehen Mühlhäusler und Harré (1990) im Rahmen ihrer Hypothese des "double indexicality“ aber auch noch eine weitere Form der Verortung durch das ICH, nämlich in einer "moral order", d.h. in einem Raum moralischer Zuständigkeit, in der der Produzent eines Textes durch den Gebrauch der ersten Person automatisch die Verantwortung für das Gesagte, d.h. dessen illokutive Kraft und perlokutiven Effekt, übernimmt (Mühlhäusler und Harré|1990: 92). Der Sprecher bzw. Autor hat über den Gebrauch der ersten Person automatisch die Verantwortung für den Wahrheitsgehalt dessen, was er äußert. Andersherum bedeutet dies entsprechend, dass die Interpretation und der Wahrheitsgehalt, der der Äußerung vom Hörer bzw. Leser beigemessen wird, abhängig von der Glaubwürdigkeit des Autors ist.

A factual statement, for instance, is labelled with the speaker as authority so that its illocutionary force is relative to the trustworthiness of that speaker. The second index again labels something other than the utterance. It labels the illocutionary force in relation to the location of the speaker in a relevant moral order, and thereby indexes the actual or prospective perlocutionary effects with the speaker as he or she who is to be held responsible for those effects. It is clear now why a use of the first person is an act of taking responsibility. (Mühlhäusler und Harré 1990: 92)

Dies macht deutlich, warum die erste Person auch und gerade im wissenschaftlichen Schreiben eine besondere Rolle spielt. Einerseits führt die Verortung in der moralischen Ordnung und die Zuweisung von Verantwortlichkeiten dazu, dass in einer positivis-

\footnotetext{
${ }^{5}$ Ethisch fragwürdige Sonderfälle, in denen beispielsweise ein Ghost Writer oder ein Mitarbeiter den Text verfasst hat, der jedoch bei der Veröffentlichung nicht genannt wird, werden hier nicht berücksichtigt. Ich gehe auch bei der Analyse meines Korpus selbstverständlich davon aus, dass sich die Autoren an die Gepflogenheiten der "Guten wissenschaftlichen Praxis"gehalten haben und ihre Texte selbständig verfasst haben.

${ }^{6}$ Mühlhäusler und Harré (1990 91) machen einen konzeptuellen Unterschied zwischen dem Vorgang der Indizierung und der Bezeichnung (,denoting') als Möglichkeiten der Verbindung zwischen sprachlichen Zeichen mit der außersprachlichen Umwelt. Das einfache Bezeichnen findet statt, wenn es eine allgemeine Bezeichnung oder einen Namen für eine Person oder ein Objekt gibt, der sowohl für den Sprecher als auch den Hörer immer die gleiche Bedeutung hat. Indizierung hingegen benutzen sie, um zu kennzeichnen, dass die Benennung einer Entität abhängig ist von ihrer Position in Bezug auf den Sprecher zu einem gegebenen Moment. Indizierung umfasst also deiktische Ausdrücke wie beispielsweise Personalpronomen.
} 
tischen Auffassung von Wissenschaft, in der die „Verantwortung" für bestimmte Gegebenheiten und Ergebnisse in den Daten und unabhängig von Personen zu suchen ist, die erste Person möglichst vollständig ausgeblendet wird. Andererseits ist es auch nachvollziehbar, dass sie ein wichtiges rhetorisches Hilfsmittel ist, um eine angemessene Autorenpersona zu konstruieren, mit der der Autor eines Textes an strategisch relevanten Stellen eben doch die Verantwortung für seine Untersuchung, seine Ergebnisse oder auch seine Interpretationen übernehmen und sich dadurch in ein bestimmtes Licht rücken kann. Dies erfordert jedoch eine gute Kenntnis der in der jeweiligen Wissenschaftskultur vorherrschenden Präferenzen, wie sich ein wissenschaftlicher Autor zu repräsentieren sollte. Und nicht zuletzt wird auch deutlich, dass mit dem Gebrauch der ersten Person immer ein gewisses Risiko einhergeht, was zu der Unterscheidung zwischen High-Risk- und Low-Risk-Funktionen der ersten Person geführt hat (Tang und John 1999; Hyland 2002a) 7

Empirische Ergebnisse zum Gebrauch der ersten Person Singular im wissenschaftlichen Schreiben haben gezeigt, dass es bedeutsame Unterschiede in englisch- und deutschsprachigen Texten gibt, die sich nicht nur auf die Häufigkeit beziehen, mit der die Pronomen benutzt werden, sondern auch die rhetorischen Funktionen dieses Pronomens im Diskurs und v. a. in der Konstruktion von Identitäten betreffen. Die Untersuchungen, die für diese Arbeit relevante Ergebnisse erbracht haben, hatten ihrerseits sehr unterschiedliche Perspektiven und Fragestellungen. DIese variieren zwischen interdisziplinären (z. B. Fløttum u. a. 2006; Hyland 2001b, 2002a, b) und interlingualen Vergleichen, wobei sowohl der Vergleich zwischen dem deutschen und dem englischen Schreiben (z. B. Hutz 1997; Kresta 1995; Kussmaul 1978; Sanderson 2008a) als auch zwischen anderen Sprachenpaaren (z. B. Fløttum u.a. 2006) berücksichtigt wird. Außerdem werden hier Arbeiten betrachtet, die Unterschiede zwischen wissenschaftlichen und studentischen Texten (z. B. Steinhoff 2007) betrachten, wobei teilweise auch L2-Texte im Fokus standen (z. B. Hyland 2002a).

Die Beschäftigung mit dem ICH-Gebrauch in wissenschaftlichen Texten, häufig auch im Vergleich zum Englischen, hat in der deutschen Forschung eine etablierte Tradition. Die überwiegende Mehrheit der Untersuchungen hat sich dabei jedoch auf die Identifizierung von Sprechakten bzw. -handlungen ${ }^{8}$ beschränkt, die über die erste Person vollzogen werden. Interaktionale Bedeutungen werden häufig, wenn überhaupt, dann nur am Rande angesprochen (Hutz 1997; Kussmaul 1978; Steinhoff 2007)9 Der weiterführende Schritt, die aus diesen Handlungen resultierenden sozialen Interakti-

\footnotetext{
${ }^{7}$ Vgl. auch die Ergebnisse von Myers (1989) zu Höflichkeit und Face-Bedrohungen im wissenschaftlichen Schreiben, S. 15

${ }^{8}$ Ich gehe in dieser Arbeit anders vor, da hier nicht die Sprechhandlungen, sondern die sprachlich dargestellten, d. h. vom Verb denotierten Handlungen betrachtet werden (s. Kap. 3.3.2. S. 71 .

${ }^{9}$ Vgl. aber Kresta (1995), der sich mit der Interpersonalität in wissenschaftlichen Texten beschäftigt hat, und Sanderson (2008a), die Autorenidentität und Autor-Leser-Beziehungen untersucht hat. In beiden Studien wurden aber dennoch nur Sprechhandlungen und nicht die sprachlich dargestellten Handlungen betrachtet.
} 
onsrollen und Identitäten zu interpretieren, wurde meines Wissens im deutschsprachigen Kontext bisher nicht unternommen (vgl. auch Adamzik 2002: 211) 10 ,

Schon 1978 hat sich Kussmaul aus übersetzungswissenschaftlicher Sicht mit dem Vergleich von deutschen und englischen Artikeln auseinandergesetzt (Kussmaul 1978). Er hat beobachtet, dass trotz einer "Abneigung gegen das Wort ,ich"“ in der wissenschaftlichen Literatur in deutschen Texten die erste Person Singular zumindest in Kombination mit Verben des Denkens und Meinens relativ weit verbreitet zu sein scheint (Kussmaul|1978 54). Sein Korpus aus geisteswissenschaftlichen „Abhandlungen“ (aus der Sprach- und Literaturwissenschaft) aus dem deutschsprachigen und englischsprachigen Raum untersuchte er in Hinblick auf die "die Konstellationen beim Kommunikationsakt thematisierenden sprachlichen Erscheinungen“, um diese zu lokalisieren und bestimmten Sprechakten zuzuordnen ${ }^{11}$, um daraufhin deren Gebrauchsregeln identifizieren zu können. Seine Untersuchung basierte auf der Annahme, dass „innerhalb bestimmter Textsorten offensichtlich feste Konventionen herrschen", dass es jedoch Unterschiede in diesen Konventionen in der deutschsprachigen und englischsprachigen Wissenschaft gebe (vgl. Kussmaul|1978, 54).

Kussmaul konzentrierte sich auf die Sprechakte Feststellungen, Ankündigungen, Rückverweise, Aufforderungen und Vermutungen. In seinen englischsprachigen Texten werden abgesehen von dem Sprechakt des Vermutens immer persönliche Wendungen in der ersten Person (Singular oder Plural) bevorzugt (Kussmaul 1978: 56), wodurch entweder der Autor in den Fokus gerückt (Singular) oder auch der Leser mit angesprochen und in den Dialog einbezogen (Plural) werde. Die deutschen Texte betonten dagegen durch das Fehlen dieser Pronomen eine unpersönliche und sachliche Haltung (Kussmaul 1978: 55). Darüber hinaus sind bestimmte Sprechakte offenbar mit spezifischen Konstruktionen gekoppelt, wobei im englischen die Ich-Konstruktionen bei allen Sprechakten außer der Vermutung bevorzugt wurden.

Als Anwendungsbereiche weist Kussmaul auch bereits darauf hin, dass diese Ergebnisse für Autoren, die in der jeweiligen Fremdsprache schreiben, eine wichtige Information darstellen:

Wer die sprachenspezifischen Konventionen einhalten und befremdliche Wirkungen vermeiden will, muß darauf achten, daß er nicht muttersprachliche Konventionen auf den in der Fremdsprache abzufassenden Text überträgt. (Kussmaul[1978, 57)

An gleicher Stelle hebt er die Relevanz dieser Untersuchung für die Übersetzungswissenschaft hervor und weist auf die Chance hin, "den oft allzu monotonen deutschen Sprachgebrauch durch eine frische angelsächsische Brise zu beleben."

\footnotetext{
${ }^{10}$ S. aber Sanderson (2008a), deren Arbeit in der deutschen Anglistik auf Englisch angefertigt wurde und die sich u. a. mit der Konstruktion von sozialen Identitäten anhand von Pronomen in geisteswissenschaftlichen Texten auseinandersetzt.

${ }^{11}$ Bei Sprechakten bezieht er sich auf John R. Searle (Kussmaul| 1978 54)
} 
Basierend auf Kussmauls Ergebnissen hat Matthias Hutz Fachzeitschriftenartikel aus der Sozialpsychologie analysiert (Hutz 1997; 235). Damit ist seine Untersuchung eine der wenigen, die sich auf nur eine Fachdisziplin konzentriert. Seine Arbeit ist in dem theoretischen Ansatz der Fachtextpragmatik verortet. In diesem Rahmen hat er ein integratives Analysemodell entwickelt (Hutz 1997: 82), mit dem er die strukturelle, die propositionale sowie die funktional-kommunikative Dimension von Fachtexten gleichzeitig beleuchtet. Dabei stellte er unter anderem die Frage nach interlingualen Gemeinsamkeiten und Unterschieden zwischen deutsch- und englischsprachigen Fachzeitschriftenartikeln (Hutz 1997: 86).

In seinem Korpus aus 30 deutschen und 30 englischsprachigen Zeitschriftenartikeln aus der Sozialpsychologie hat Hutz u. a. den Gebrauch von Verfasserreferenzen untersucht (vgl. Hutz 1997; 231-238), indem er die Personalpronomen der ersten Person sowie die unpersönlichen Pronomen MAN und ONE je in den einzelnen Teiltexten ermittelt und mit der Anzahl der finiten Verben ins Verhältnis gesetzt hat. Hutz sieht die Ergebnisse von Kussmaul, dass in englischsprachigen Texten öfter die erste Person verwendet wird als in deutschen, auch in seinen Ergebnissen bestätigt. Allerdings ist der Unterschied zwischen seinen beiden Teilkorpora nicht so groß wie bei Kussmaul. In seinen englischen Texten werden 1,5\% der finiten Verbformen mit dem Subjekt in der ersten Person realisiert, während es in den deutschen ca. 1,0 \% sind.

Als eine Erklärungsmöglichkeit für diesen recht geringen Unterschied nennt Hutz die zunehmende Orientierung an angloamerikanischen Schreibkonventionen im Fach Sozialpsychologie, die möglicherweise dazu führe, dass die Darstellungsweise in wissenschaftlichen Fachartikeln (zumindest in diesem Fach) persönlicher werde. Er weist aber auch darauf hin, dass diese Frage aus seiner Untersuchung heraus nicht beantwortet werden kann, da es hierzu diachronisch ausgerichteter Studien bedarf ( 1997. 237).

Weiter differenzierend stellt Hutz fest, dass die erste Person Singular in seinen deutschen Texten gar nicht vorkommt, dass aber statt dessen vereinzelt die erste Person Plural als pluralis maistatis bzw. modestiae benutzt wird, auch in Texten, die von einem Einzelautor verfasst wurden. Demgegenüber liegt der Anteil der finiten Verben mit Subjekten in der ersten Person Singular in den amerikanischen Artikeln seines Korpus bei 10,9\%, obwohl nur ca. ein Drittel dieser Texte von einem Einzelautor stammen.

Die Subjekte der ersten Person werden nach Hutz' Analyse besonders häufig für die Formulierung von Zielsetzungen und Hypothesen (in Einleitungen), die Darstellung experimenteller Abfolgen (im Methodenteil) sowie statistische Auswertungsverfahren (im Ergebnisteil) und die Zusammenfassung von Ergebnissen (im Diskussionsteil) verwendet (Hutz 1997; 237). Zwar sind dies alles Handlungen, bei denen in den meisten Fällen der Autor auch eindeutig als Handelnder identifiziert werden kann, aber dennoch, oder auch gerade deswegen, ist der Gebrauch der ersten Person in diesen Zusammenhängen immer fakultativ (Hutz 1997: 237), so dass hier angenommen 
werden kann, dass der Auswahl des Subjekts kulturgebundene Schreibkonventionen zugrundeliegen.

Mit der Interpersonalität in linguistischen Fachtexten setzte sich Ronald Kresta (1995) intensiv auseinander. Interpersonalität versteht er darin als eine

in allen sprachlichen Äußerungen vorhandene Erscheinung, die eine Markierung der kommunikativen Rolle durch den Produzenten ermöglicht. Diese kommunikative Rolle schließt ein: 1) die Identifikation bzw. Nicht-Identifikation des Produzenten und die Kundgabe seiner Meinungen, Urteile, Kommentare usw. (= Sprecherbezogenheit) und 2) das Sprachverhalten des Produzenten gegenüber seinen Rezipienten, z. B. das Ansprechen oder Fragen (= Hörerbezogenheit). Sprachliche Realisierungsformen dieser Erscheinung sind theoretisch auf allen sprachlichen bzw. textuellen Ebenen zu beobachten. (Kresta|1995: S. 36)

Krestas Korpus umfasst 160 linguistische Fachtexte, jeweils 80 deutsche und 80 englische. In diesen je 80 Texte sind zu gleichen Anteilen Aufsätze, Rezensionen, Entgegnungstexte und Auszüge aus Einführungen in die Linguistik enthalten. Im Folgenden werden nur seine Ergebnisse zum Vergleich der Aufsätze berücksichtigt.

Kresta konnte ebenfalls bestätigen, dass das ICH in englischen Aufsätzen häufiger, d.h. etwa doppelt so häufig, benutzt wird, wie in deutschen (Kresta 1995: 168). Dabei hat er fünf Sprechhandlungen identifiziert, die in der ersten Person Singular umgesetzt werden: das Strukturieren des Textes, das Untermauern der vertretenen Thesen, das Berichten, die Angabe metalingualer Äußerungen und die Danksagung.

Einen weiteren wichtigen Bereich für die Anwendung und auch die Analyse des Ich-Gebrauchs in wissenschaftlichen Artikeln sieht Kresta bei der Meinungsäußerung. Mit der Struktur [I/ich + Verb des Meinens] drücken die Autoren wissenschaftlicher Texte seinen Ergebnissen zufolge am häufigsten ihre Meinung aus. Die Anwendung dieser Struktur ähnelt sich in den deutschen und den englischen Aufsätzen dabei sehr (Kresta|1995 241).

Die Arbeit von Tamsin Sanderson, die in der deutschen Anglistik erschienen ist, kombiniert die diskursanalytische mit der korpuslinguistischen Perspektive. Sandersons Korpus, das Synchronic Corpus of English and German Academic Discourse (SCE$G A D$ ) besteht aus 100 Texten, von denen 25 von britischen, 25 von US-amerikanischen und 50 von deutschen Autoren verfasst wurden (Sanderson|2008a; 65). Es enthält Texte, die überwiegend von Einzelautoren geschrieben und in führenden Zeitschriften der entsprechenden Fächer veröffentlicht wurden (Sanderson 2008a: 68-69).

Im Gegensatz zu vielen anderen Studien zu Unterschieden im wissenschaftlichen Schreiben hat Sanderson also nicht nur nach deutsch- und englischsprachigen Autoren unterschieden, sondern auch nationalspezifisch weiter differenziert. Dies ist ins- 
besondere sinnvoll, da sie in ihrer Arbeit den Kulturbegriff als rein nationalspezifisch auffasst.

Sanderson untersucht den möglichen Einfluss verschiedener Variablen wie (National-)Kultur, Gender, Disziplin und akademischem Status auf das akademische Schreiben in den geisteswissenschaftlichen Disziplinen Philosophie, Geschichte, Folklore (Studies)/Volkskunde, Literaturwissenschaft und Linguistik (Sanderson|2008a: 64). Sie betrachtet dabei das wissenschaftliche Schreiben als von Natur aus soziale Tätigkeit und untersucht daher "how academic texts reflect, encode and construct a complex network of social identities, relations and interactions." (Sanderson 2008a: 65).

Sandersons Ergebnisse zeigen ebenfalls, dass, obwohl das Deutsche und das Englische ähnliche sprachliche Mittel für Personal- und Selbstreferenzen zur Verfügung stellen, ihr Gebrauch bei Autoren, die in diesen Sprachen schreiben, doch sehr unterschiedlich ist, dass diese Autoren also ihre soziale Interaktion mit den Lesern und der Community auf unterschiedliche Weise strukturieren (vgl. Sanderson|2008a: 160). Englischsprachige Autoren geisteswissenschaftlicher Texte treten sehr viel offener in ihren Texten auf als deutschsprachige, sie sind deutlicher sichtbar und treten in ihren Texte explizit in den Dialog mit ihren Lesern (vgl. Sanderson 2008a: 161). Deutschsprachige Autoren in Sandersons Korpus halten sich dagegen offenbar noch stärker an das „Ich-Tabu“.

Darüber hinaus verwenden deutsche Autoren auch und gerade in Texten, die nur von einem Einzelautor verfasst wurden, die Plural-Formen, um ihre Mitgliedschaft in einer Gruppe, in diesem Fall der Scientific Community, darzustellen, während englischsprachige Autoren eher zu der individuelleren Darstellung im Singular tendieren. Dadurch, so Sanderson (Sanderson 2008a 161), wirken Texte von englischsprachigen Autoren insgesamt individueller und gleichzeitig persönlicher als Texte von deutschsprachigen Autoren. In Bezug auf Unterschiede zwischen britischen und amerikanischen Autoren hat Sanderson (Sanderson 2008a: 161) herausgefunden, dass in amerikanischen Texten personale Referenzen noch etwas häufiger vorkommen als in britischen. Für diesen Trend konnte sie jedoch keine statistische Signifikanz nachweisen (s. Sanderson 2008a: 161).

Für die Variablen Sprache, Kultur, Disziplin, Gender und Alter konnte Sanderson zeigen, dass sie einen statistisch signifikanten Einfluss auf den Gebrauch und die Häufigkeit von Personalpronomen haben. Dabei ist jedoch zu beachten, dass diese Variablen nicht unabhängig voneinander wirken, sondern miteinander interagieren und entsprechend auch nicht unabhängig voneinander als Erklärung für die untersuchten linguistischen Phänomene herangezogen werden sollten:

No one variable can completely explain linguistic behaviour; rather, written production is a multicausal phenomenon, influenced by a number of considerations. (Sanderson 2008b; 64) 
Selbstreferenz anhand von Personlpronomen wird in Sandersons Korpus zu unterschiedlichsten und teilweise auch gegensätzlichen Zwecken benutzt, beispielsweise um die Verantwortung des Autors für eine Behauptung entweder zu verstärken oder abzuschwächen. Genauso kann Selbstreferenz einerseits ein Zeichen der Bescheidenheit sein, um zu zeigen, dass der Autor allein eine bestimmte Meinung hat und nicht für andere mit sprechen kann und will, andererseits kann sie auch als vielleicht unangemessenes „Eigenlob“ gewertet werden (vgl. Sanderson 2008a. 160).

Sanderson (2008b 77) hat elf verschiedene Funktionen der Personalpronomen im Singular und Plural unterschieden. In ihrem Korpus werden sie benutzt, um persönliche Meinungen und Kommentare abzugeben (Personal opinion), um den Text zu kommentieren (Text comments), um die Zugehörigkeit zu einer bestimmten Gruppe zu demonstrieren (Group: academics, group: author $\mathcal{E}$ reader, group: humans, group: other), um die Methodologie darzustellen (Methodology), um die Leser direkt anzusprechen (Address reader, hauptsächlich in der zweiten Person), um andere Arbeiten des Autors hervorzuheben (Self-advertisment), um Dank auszudrücken (Acknowledgement) sowie um auf Quellen anderer Autoren zu verweisen (Reference to secondary sources).

Dabei konnte sie deutliche Unterschiede zwischen den beiden Sprachgruppen feststellen. Während die englischsprachigen Autoren personale Referenzen vorwiegend dazu benutzten, persönliche Meinungen zu äußern sowie ihren eigenen Text zu kommentieren, neigten deutsche Autoren dazu, sich selbst als Teil der ganzen Menschheit darzustellen (wir (alle) Menschen). Die deutschen Autoren verfolgen mit diesem human we nach Sanderson keinen offenkundigen interaktiven Zweck, im Gegensatz zu den englischsprachigen Autoren, deren Verwendung von Personalreferenzen eindeutig als interaktiv zu interpretieren ist (Sanderson 2008b: 78). Sanderson glaubt sogar, ein gewisses „Unwohlsein“ bei den deutschen Autoren feststellen zu können, wenn diese doch einmal eine persönliche Meinung in der ersten Person Singular äußern, was sie auf das „Ich-Tabu“ (Weinrich 1989, 132) in wissenschaftlichen Texten zurückführt (Sanderson 2008b; 59).

Besonders häufig werden Personalreferenzen der ersten Person in Sandersons Korpus in denjenigen Textteilen verwendet, in denen der Autor sein methodologisches Vorgehen beschreibt. Insbesondere in den Texten des Faches Volkskunde/Folklore Studies, in denen ethnographische Untersuchungen ${ }^{12}$ beschrieben werden, würden Selbstreferenzen besonders häufig gebraucht, was häufig sogar in eine beinahe autobiographische Beschreibung der Erfahrungen des Autors münde (Sanderson 2008b 80). In erster Linie kann das natürlich auf die besonders intensive Methode der Feldforschung zurückgeführt werden, in der der Forscher unter Umständen voll in die zu untersuchende Gruppe integriert wird und so in seinem Text tatsächlich sein eigenes Leben mit seinem „Untersuchungsgegenstand“ beschreibt. Aber auch hier neigen die deut-

\footnotetext{
${ }^{12}$ Diese Methode ist auch in der Soziologie gebräuchlich und liegt einigen der in der vorliegenden Arbeit untersuchten Texte zugrunde.
} 
schen Autoren im deutlichen Gegensatz zu den englischsprachigen eher zu unpersönlichen Darstellungen in der dritten Person, was Sanderson als besonders merkwürdig (,awkward“, Sanderson (2008b 80)) empfindet, da die dritte Person auch bei sehr persönlichen Beschreibungen z. B. der Kleidung des Autors verwendet wird (Sanderson 2008b 80) ${ }^{13}$

Den neuesten und wohl auch umfassendsten Beitrag zur Untersuchung des ICH in deutschen Wissenschaftstexten hat Torsten Steinhoff geleistet, allerdings hat er nicht das Deutsche mit dem Englischen verglichen. Er hat sich vielmehr mit dem Vergleich von studentischen Arbeiten mit veröffentlichten Texten auseinandergesetzt, um die Entwicklung wissenschaftssprachlicher Kompetenzen nachzuzeichnen.

Dafür hat hat er drei Korpora untersucht, von denen er zwei selber zusammengestellt hat. Sein studentisches Korpus umfasst insgesamt 296 Hausarbeiten aus den Fächern Geschichtswissenschaft, Literaturwissenschaft, Linguistik, Soziologie, Philosophie, Erziehungswissenschaft, Politikwissenschaft, Deutsch als Fremdsprache, Psychologie (Steinhoff 2007 153-154) und sein Expertenkorpus 99 Texte, die zu gleichen Teilen aus Fachzeitschriften der Fächer Geschichtswissenschaft, Literaturwissenschaft und Linguistik gewählt wurden (Steinhoff 2007: 156). Außerdem hat er journalistische Texte aus Cosmas II (IDS) mit berücksichtigt (Steinhoff|2007; 157).

Darüber hinaus hat er Experteneinschätzungen von Wissenschaftlern aus den genannten Disziplinen vornehmen lassen (Steinhoff 2007: 157). Diese sollten kurze Ausschnitte aus den Korpora nach ihrer subjektiv empfundenen Wissenschaftlichkeit einstufen. Daneben hatten sie auch die Möglichkeit, diese Ausschnitte zu kommentieren (vgl. Steinhoff 2007; 159).

Steinhoff hat drei Autorenrollen identifiziert, die durch die erste Person Singular realisiert werden: das Verfasser-Ich, das Forscher-Ich und das Erzähler-Ich (Steinhoff 2007 180-204). Das Verfasser-Ich tritt v. a. in Passagen der Textkommentierung auf, d.h. in Wendungen, die der Lesersteuerung dienen. Weiterhin fasst Steinhoff darunter auch Textprozeduren, die Intertextualität herstellen (zitieren, verweisen etc.) sowie Danksagungen. Das Forscher-Ich dient u. a. dazu, Originalität zu bezeugen. Es wird v. a. für Begriffsbildungen und Definitionen eingesetzt, aber auch um Hypothesen zu explizieren sowie vereinzelt auch, um sich kritisch über andere Autoren bzw. deren Annahmen zu äußern. Das Erzähler-Ich dagegen wird nur von Studierenden in den frühen Phasen ihres Studiums verwendet. Es wird benutzt zur Auto-Narration, d.h. die Autoren erzählen von sich selbst und ihren Erfahrungen und Empfindungen,

\footnotetext{
${ }^{13}$ Hier sei noch hinzugefügt, dass die Methodenteile in wissenschaftlichen Texten unabhängig vom Fach und der jeweils angewandten Methode auch immer dazu dienen, den Autor in der wissenschaftlichen Gemeinschaft zu verorten und seine Zugehörigkeit dadurch zu signalisieren, dass er in seinem Text darstellt, wie und warum er welche Methoden benutzt. Dadurch kann er zeigen, dass er ein vollwertiges Mitglied dieser Gemeinschaft ist, da er die Arbeitsformen und Instrumente seines Faches kennt und angemessen anwendet.
} 
die sich v. a. auf die Hausarbeit beziehen. Diese Form wird in den Kontextualisierungsversuchen als eindeutig nicht wissenschaftlich eingeschätzt.

Steinhoff konnte in seiner Studie zeigen, dass die erste Person Singular in deutschen Texten sowohl von Wissenschaftlern als auch von Studierenden benutzt wird. Unterschiede zwischen den Disziplinen lassen sich dahingehend feststellen, dass das ICH am häufigsten in linguistischen Texten verwendet wird, weniger in literaturwissenschaftlichen Texten und am seltensten in der Geschichtswissenschaft. Aus der Perspektive der Schreibentwicklung sei nach Steinhoff auffällig, dass Studierende am Anfang ihres Studiums das ICH wesentlich häufiger und auch in anderen rhetorischen Zusammenhängen benutzten als wissenschaftliche Autoren. Im Laufe der fortschreitenden Sozialisation nähere sich in den fachspezifischen wissenschaftlichen Kontext der ICH-Gebrauch in studentischen Texten jedoch dem in wissenschaftlichen Artikeln an. Dabei würden auch die typischen disziplinspezifischen Unterschiede erkennbar.

In der norwegischen KIAP-Studie untersuchen Kjersti Fløttum, Trine Dahl und Torodd Kinn insgesamt 450 englische, französische und norwegische wissenschaftliche Artikel aus drei Disziplinen (Linguistik, Ökonomie, Medizin), die zwischen 1992 und 2003 publiziert wurden. Sie verfolgen die Fragestellung, ob es so etwas wie kulturelle Identitäten im wissenschaftlichen bzw. akademischen Diskurs tatsächlich gibt und wenn ja, wie stark diese an die Disziplin oder an die nationale Sprache gebunden sind (Fløttum u.a. 2006, 1). Ihr wichtigstes Ergebnis ist, dass in Bezug auf die kulturelle Identität im wissenschaftlichen Diskurs die Disziplin, der der Autor angehört, offenbar wichtiger sei als seine Sprache. In diesem Zusammenhang haben sie vier verschiedene rhetorische Autorenrollen identifiziert, anhand derer sie das explizite Auftreten der Autoren in ihren Texten kategorisieren. Diese sind die Rolle als schreibender (writer role), als forschender (researcher role), als argumentierender (arguer role) und als (be)wertender (evaluator role) Autor (Beispiele s. S. 75).

Beim Vergleich der Vorkommenshäufigkeit dieser Rollen stellte sich heraus, dass die dominanteste Rolle die researcher role war, die ca. 50\% aller Fälle ausmachte. Ebenfalls relativ häufig wurde die erste Person Singular in der writer role umgesetzt (ca. $25 \%$ ). Die arguer role hingegen umfasst nur ca. $14 \%$ der ICH-Vorkommen, was die Autoren überraschte:

Given the well-known competitive situation within research, we might have expected a more explicit author manifestation by an arguer role combined with the I presence. (Fløttum u. a. 2006: 91)

Die evaluator role tritt nur selten und nur in einigen wenigen Artikeln auf, Fløttum u.a. (2006) betrachten sie daher auch eher als stilistisches Merkmal einzelner Texte, aber als nicht so relevant für eine generelle Charakterisierung wissenschaftlicher Artikel (Fløttum u.a. 2006: 91). Bezüglich der sprachlichen Unterschiede haben sie festgestellt, dass 
der englischsprachige wissenschaftliche Stil expliziter und direkter sei als der norwegische und der französische, was auch die Ergebnisse anderer vergleichender Studien bestätige (Fløttum u. a. 2006. 92). Die disziplinspezifischen Unterschiede gestalteten sich derart, dass insbesondere die arguer role in linguistischen Texten häufiger auftrete als in wirtschaftswissenschaftlichen Texten. In medizinischen Texten werde die erste Person Singular in allen Rollen vergleichsweise selten verwendet (Fløttum u. a. 2006 92-93), was auch mit Ken Hylands Ergebnissen zum Unterschied zwischen hard und soft disciplines korrespondiert (z. B. Hyland 2000, 2003, 2008).

Ken Hyland hat sich in mehreren Artikeln (vgl. z. B. Hyland 2001b, 2002a) mit der Verfasserreferenz in wissenschaftlichen und studentischen Arbeiten beschäftigt. Sein wissenschaftliches Korpus setzt sich aus 240 englischsprachigen Forschungsartikeln (Research Articles) aus acht verschiedenen Disziplinen zusammen (jeweils drei Texte aus den zehn führenden Journals dieser Disziplinen), so dass die sogenannten hard und soft sciences, also naturwissenschaftliche Fächer und geistes- und sozialwissenschaftliche Fächer repräsentiert sind. Als hard sciences deklariert Hyland dabei die Disziplinen Physik, Biologie, Maschinenbau und Elektrotechnik, zu den soft sciences zählt er Marketing, angewandte Linguistik, Soziologie und Philosophie (Hyland 2008: 13).

Außerdem hat er ein studentisches Korpus aus 64 Abschlussarbeiten auf Undergraduate-Niveau bearbeitet. Diese wurden an der Universität von Hong Kong in denjenigen Fächern geschrieben, die den Disziplinen, aus denen die Texte für das wissenschaftliche Korpus gewählt wurden, am nächsten kommen (vgl. Hyland 2002a: 1096). Diese Arbeiten wurden von chinesischen Studierenden in Englisch verfasst, Hylands Fokus lag hier also, anders als bei den bisher zitierten Arbeiten auf dem Schreiben in der Fremdsprache.

Hyland kam in seinen Untersuchungen zur Verfasserreferenz zu dem Ergebnis, dass Selbstreferenzen des Autors in jedem der Artikel seines Korpus zu finden gewesen seien (Hyland 2001b 212), dass dabei jedoch gravierende Unterschiede in der Menge und der Umsetzung zwischen den von ihm untersuchten Disziplinen zu erkennen seien (Hyland 2001b 212). Bezüglich der Unterscheidung in hard und soft disciplines war dabei auffällig, dass ca. $69 \%$ aller Selbstreferenzen in den Geistes- und Sozialwissenschaften auftraten, überwiegend in Form von Pronomen der ersten Person (Hyland 2001b 213). Diese Unterschiede erklärt Hyland mit Unterschieden in der Art der gewonnenen Erkenntnisse und der entsprechenden Methoden in den hardund soft sciences. Der positivistische und objektivistische Anspruch der Naturwissenschaften führe dazu, dass die untersuchten Phänomene und die allgemeine Relevanz der Ergebnisse in den Vordergrund rücke und die Person des Autors weniger wichtig für die Argumentationslinie werde. Die Gegenstände der Geistes- und Sozialwissenschaften hingegen seien im Allgemeinen weniger eindeutig messbar und die Ergebnisse unterliegen weit mehr der Interpretationsfähigkeit des jeweiligen Wissenschaftlers. Die Fähigkeit, ein glaubwürdiges und angemessen autoritatives Bild von sich selbst zu 
entwerfen, sei daher für einen Autor in diesen Disziplinen von herausragender Bedeutung:

Establishing an appropriately authorial persona and maintaining an effective degree of personal engagement with one's audience are valuable strategies for probing relationships and connections between entities that are generally more particular, less precisely measurable, and less clear-cut than in the hard sciences. [...] Successful communication depends to a larger extent on the author's ability to invoke a real writer in the text. Self-mention can help construct an intelligent, credible, and engaging colleague, by representing an authorial self firmly established in the norms of the discipline and reflecting an appropriate degree of confidence and authority. (Hyland 2001b: 216)

Die wichtigste Form der Selbstbenennung in den untersuchten englischsprachigen wissenschaftlichen Texten sind offenbar die Pronomen der ersten Person Singular und Plural, insbesondere, wenn sie in Subjekt-Position vorkommen (Hyland 2001b: 215). Englischsprachige Autoren in den soft disciplines benutzen diese Selbstreferenzen v. a. um ihre eigenen Annahmen, Erkenntnisse und Interpretationen zu präsentieren und so ihren Beitrag zum wissenschaftlichen Fortschritt kenntlich zu machen. Basierend auf der Auswertung seiner Korpora hat Hyland fünf Diskursfunktionen identifiziert, in denen überwiegend die erste Person benutzt wird, um die Rolle des Autors im jeweiligen Kontext zu kennzeichnen (Hyland2002a: 1098-1106): Expressing self-benefits, Stating a purpose/goal, Explaining a procedure, Elaborating an argument, Stating results/claims.

Die Unterschiede zwischen wissenschaftlichen und studentischen Texten, die sich in der Umsetzung dieser Funktionen mit der ersten Person Singuler ergeben haben, erklärt Hyland mit Bezug auf Unterschiede zwischen low stake- und high stake-Funktionen. Die Studierenden, deren Arbeiten Hyland untersucht hat, vermieden die erste Person dort, wo sie von den erfahrenen Autoren bevorzugt benutzt wurde, v. a. in Zusammenhängen, in denen sie Ergebnisse interpretieren, eigene Behauptungen aufstellen und für diese argumentieren (Hyland 2002a: 1103). Das heißt nicht, dass sie diese Prozeduren nicht durchführten, jedoch schienen sie hier bewusst die direkte Referenz auf sich selber zu vermeiden, um sich möglichst nicht angreifbar zu machen (Hyland 2002a: 1107). Hyland interpretiert ihre Präferenz für unpersönliche Wendungen hier dahingehend, dass die studentischen Autoren ihre Objektivität hervorheben wollten, weil sie entweder unsicher waren und Angst hatten, sich zu irren oder nicht die Meinung des Prüfers zu treffen. Ein weiterer Grund sei möglicherweise Bescheidenheit, weil sie glaubten, dass es ihnen in ihrem Status als Studierenden nicht zustehe „sich selbst so wichtig zu machen“(Hyland 2002a: 1105). 


\subsubsection{1. Person Plural}

Die Pronomen der ersten Person Plural zeichnen sich durch eine wesentlich größere innere Komplexität aus, als die der ersten Person Singular (Fløttum u. a. 2006: 95). Das WIR bezeichnet neben dem Sprecher (oder dem Autor) noch eine beliebige Anzahl weiterer Personen, in die die Hörer (Leser) inkludiert sein können oder auch nicht. Daraus ergeben sich die übergeordneten Lesarten des inklusiven und des exklusiven WIR und es wird deutlich, dass das WIR nicht als reiner ICH-Ersatz betrachtet werden kann (vgl. Steinhoff 2007: 207). Die Entscheidung für das WIR und damit gegen das ICH kann unterschiedliche Beweggründe haben:

The reasons why academic authors would wish to reduce or avoid the subjectivity signaled by first person singular forms are varied. On the one hand, deciding not to foreground oneself may be regarded as a form of modesty through a desire to shrink into the background, emphasising the factual content of the article rather than its originator. On the other hand, couching personal opinions and actions in plural pronoun could also be a strategy for increasing the gravity of these opinions, and underlining one's own importance: it is not for nothing that the second person singular polite forms of address in many languages are grammatically plural forms [...]. (Sanderson 2008a: 153)

Auch das WIR stand schon mehrmals im Fokus von Untersuchungen zum wissenschaftlichen Schreiben. Matthias Hutz (zur Vorstellung der Studie s. S. 51) hat beispielsweise in seiner Untersuchung der ersten Person Plural drei unterschiedliche Verwendungsweisen festgestellt (Hutz 1997, 234-235). Das WIR kann erstens den Autorenplural bzw. das collective we kennzeichnen, wenn es sich um einen Text handelt, der von einem Autorenkollektiv verfasst wurde ${ }^{14}$. In einigen Fällen wird diese Form jedoch auch verwendet, wenn es sich um einen Text handelt, der von einem Einzelautor verfasst wurde (Hutz 1997; 234). Dieses WIR entspricht dem exklusiven WIR und Hutz wertet die Verwendung als pluralis maiestatis bzw. pluralis modestiae, wobei er letzteres für wahrscheinlicher hält (Hutz 1997: 234).

Eine weitere Funktion des WIR sieht Hutz auf Basis seiner Untersuchung in der Kennzeichnung eines impliziten Dialogs, was er in seiner Auswertung als inclusive we bezeichnet. Hier ist spezifisch, dass der Leser vom Autor in den dargestellten Denkprozess bzw. die Argumentation mit einbezogen wird. Als letzte Form des WIR-Gebrauchs sieht Hutz die Kennzeichnung einer kollektiven Erfahrung, das generalizing we, das neben dem Verfasser des Textes und dem Leser auch die breite Öffentlichkeit mit einschließt. Dieses WIR wird in verschiedenen anderen Untersuchungen noch weiter differenziert (s. u.).

\footnotetext{
${ }^{14}$ In dem in dieser Arbeit untersuchten Korpus ist allerdings kein Text enthalten, der von mehreren Autoren verfasst wurde.
} 
Während die erste Person Singular in Hutz' deutschen Texten gar nicht vorkommt, wird die erste Person Plural dort vereinzelt benutzt. Dass sie auch in Texten, die von einem Einzelautor verfasst wurden, vorkommt, deute auf die Bedeutung des WIR als pluralis maistatis bzw. modestiae hin (Hutz 1997. 237). Beim Gebrauch des WIR wird in beiden Sprachen offenbar eindeutig der exklusive Autorenplural, mit dem sich die Autoren des jeweiligen Artikels selber benennen, bevorzugt, während das inklusive WIR mehr von deutschen Autoren als von amerikanischen verwendet wird, was den deutschen Texten „mitunter einen etwas belehrenden Charakter verleiht" (Hutz 1997: 237). Das generalizing we spielt nach Hutz' Angaben weder in den deutschen noch in den amerikanischen Texten eine Rolle (Hutz 1997; 237).

Torsten Steinhoff, der sich in seiner Analyse der ersten Person Plural stark an Ronald Kresta und dessen Kategorisierungen des WIR anlehnt (s. Kresta 1995), unterteilt das inklusive WIR in vier Kategorien, nämlich das Autoren-WIR, das Teamwork-WIR, das Fachkreis-WIR und das Gemeinschafts-WIR (Steinhoff 2007; 207). Dabei entspricht das „Teamwork-WIR“ Hutz' inclusive we und umfasst neben dem Autor die Leser des Textes, die die beschriebenen Handlungen gemeinsam vollziehen (Steinhoff 2007: 206). Hutz' Kategorie des generalizing we wird äquivalent unterteilt in das "Fachkreis-WIR“, mit dem die scientific community angesprochen wird, und in das "Gemeinschafts-WIR", das einen noch größeren Skopus hat und die weiterreichende Gemeinschaft bis hin zur gesamten Menschheit bezeichnen kann (vgl. Steinhoff 2007: 207).

Bezüglich der Verwendungshäufigkeit konnte Steinhoff zeigen, dass die Autoren der von ihm untersuchten wissenschaftlichen Texte (s. auch S. 55) am häufigsten das Teamwork-WIR (39,9 \% seiner Belege) und am zweithäufigsten das GemeinschaftsWIR (25,2\%) benutzen. Die eindeutige Selbstreferenz anhand des exklusiven AutorenWIR (,Mitverfasser-WIR“ nach Kresta (1995: 291) ${ }^{15}$ ) ist nur die dritthäufigste Variante $(23,4 \%)$, während das Gemeinschafts-WIR am seltensten benutzt wird (5,0\%). Die restlichen 6,5\% seiner Belege konnte Steinhoff nicht eindeutig in eine dieser Kategorien einordnen (Steinhoff 2007; 215-216).

Im Vergleich zum Autoren-WIR wird das ICH in seinem Korpus zwar etwa dreimal häufiger gebraucht, aber was die Funktionen im Text betrifft, sind diese beiden Formen der Verfasserreferenz nach Steinhoffs Analyse vergleichbar. Genau wie das ICH wird auch das Autoren-WIR vorrangig zur Textkommentierung, zur Gegenstandskonstitution oder zur Begriffsbildung verwendet, einen mit dem Erzähler-ICH vergleichbaren Gebrauch hat Steinhoff allerdings nicht festgestellt (Steinhoff 2007: 216-217).

Das Teamwork-WIR ist das am häufigsten benutzte WIR in Steinhoffs Texten und dient dazu, die Beziehung zu und die Zusammenarbeit mit dem Leser in den Vordergrund zu stellen.

\footnotetext{
${ }^{15}$ Kresta konnte diese Verwendungsweise nur in einem Text der von ihm untersuchten Aufsätze finden, ansonsten kam dieses WIR in seinem Gesamtkorpus überwiegend in Entgegnungen zum Einsatz (Kresta 1995 292) .
} 
Der Leser wird zum Mit-Denkenden und Mit-Handelnden gemacht. Er, partizipiert' an der Analyse des empirischen Materials [...] und an bestimmten Schlussfolgerungen [...]. (Steinhoff 2007; 220)

Dies hat vor allem den Effekt, dass die Aussagen, die so getroffen werden, suggerieren, dass der Leser sie mit vollzogen hat, wodurch sie besonders überzeugend wirken (vgl. Steinhoff 2007: 220). Kresta (1995), auf dessen Kategorien Steinhoffs Analyse der WIRReferenzen basiert, hat als wichtigste Verben, die mit diesem Teamwork-WIR kombiniert werden und mit denen entsprechend diese enge Beziehung zwischen Autor und Leser etabliert wird, für das Englische see, note, consider, find, examine, come und für seine deutschen Texte sehen, betrachten, feststellen sowie kommen identifiziert. Mit dem Teamwork-WIR werden in Krestas Aufsätzen die Sprech- bzw. (bei Kresta) Sprachhandlungen Untermauern einer These und Textstrukturieren umgesetzt, genauso wie er es für seine Vorkommen vom Autoren-WIR herausgefunden hat (Kresta 1995; 296).

Die nächstgrößere Gruppe, die üblicherweise in einem wissenschaftlichen Text mit WIR benannt werden kann, ist der sog. Fachkreis (Steinhoff 2007: 223), d. h. die Angehörigen einer Disziplin oder eines Faches, in dem hier untersuchten Korpus entsprechend alle Soziologen oder etwas weiter gefasst alle Sozialwissenschaftler. Da der angenommene Leser im Normalfall dieser Fachdisziplin angehört, ist er auch hier wieder inkludiert, Kresta benennt die Mitglieder dieser Gruppe bzw. deren Beziehung zueinander entsprechend als „Ich \& Du \& Müllers Kuh“ (Kresta 1995: 299).

Während dieses WIR in Steinhoffs - ausschließlich deutschen - Texten am seltensten vorkam (Steinhoff 2007: 223), konnte Kresta beim Vergleich mit dem Englischen feststellen, dass das WE in dieser Funktion beinahe doppelt so häufig benutzt wurde wie das WIR (Kresta 1995: 300).

Andere Untersuchungen zu englischsprachigen Texten haben ähnliche Ergebnisse erzielt. Kuos Analyse von Personalpronomen in Texten aus dem natur- und ingenieurswissenschaftlichen Bereich hat gezeigt, dass die Pronomen der ersten Person Plural auch hier die am häufigsten verwendeten sind. Die erste Person Singular dagegen konnte sie in ihren 36 Texten (von denen jedoch nur drei von einem Einzelautor verfasst waren) gar nicht nachweisen (Kuo 1999: 124).

Auch Kuo unterscheidet zwischen inklusivem und exklusivem Gebrauch von WIR und konnte auch Ausprägungen von WIR (bzw. WE) nachweisen, die sich auf die Fachcommunity beziehen, welche sie als nicht auf den Autor bzw. den Autor und den Leser bezogen interpretiert hat. Da sich Kuo mit Texten aus den hard sciences beschäftigt hat, sind die quantitativen Ergebnisse dieser Untersuchung hier nicht weiter relevant, da nicht direkt vergleichbar. Die verschiedenen Diskursfunktionen, die das WIR in Kuos Texten erfüllt hat, sollen jedoch kurz betrachtet werden. Unter Diskursfunktion versteht Kuo dabei: 
[...] the function that a sentence containing a personal pronoun performs in the immediate discourse context of a journal article. It reflects the specific communicative purpose of writers-researchers in a certain part of a journal article. (Kuo|1999: 130)

Kuo hat gezeigt, dass das WIR in ihren Texten überwiegend exklusiv, d. h. mit Referenz auf den Autor benutzt wird und überwiegend die Funktion „explainig what was done" erfüllt (Kuo 1999; 131). Dadurch werde die Person des Autors und seine Rolle im Forschungsprozess hervorgehoben, was ihrer Meinung nach im Gegensatz zur eigentlich erwarteten bzw. unterstellten Fokussierung auf die Untersuchung selbst steht und dem Autor dazu dient, sich innerhalb seines Artikals zu positionieren (Kuo|1999 132, 136). Als weitere Diskursfunktionen, die das exklusive WIR erfüllt, konnte sie die folgenden nachweisen:

- proposing a theory or approach,

- stating a goal or purpose,

- showing results or findings,

- showing commitment or contribution to research,

- comparing approaches or viewpoints,

- expressing wish or expectation (Kuo|1999: 132)

Am zweithäufigsten konnte sie das inklusive WIR mit Bezug auf den Autor und den Leser nachweisen, das vor allem dazu benutzt wird, das grundlegende Wissen sowie Ziele und Annahmen so darzustellen, dass sie vom Leser als gemeinsam und geteilt wahrgenommen werden (Kuo 1999 : 132).

By using inclusive we, which involves both writers and readers in the same activity, the writers presuppose readers' background knowledge and ability to follow the arguments. The use of inclusive we shortens the distance between writers and readers and stresses solidarity with readers. (Kuo 1999: 132-133)

Für die inklusiven WIR hat sie die folgenden Diskursfunktionen als besonders relevant identifiziert, wobei die letzten beiden auch von dem WIR, das sich auf die Fachgemeinschaft bezieht, erfüllt werden:

- justifying a proposition,

- hedging a proposition or claim,

- giving a reason or indicating necessity (Kuo|1999: 133)

Allerdings hat sie in Bezug auf die Verteilung dieser Pronomen auf die Texte in ihrem Korpus große Variationen festgestellt (Kuo|1999: 133).

Auch Tamsin Sanderson konnte in ihrem SCEGAD-Korpus zeigen, dass die erste Person Plural weit mehr von Deutschen Autoren benutzt wurde als von englischsprachigen (Sanderson 2008a: 161). Sie hat die wesentlichen Funktion von Personalpronomen bei der Konstruktion von Autorenidentität(en) untersucht und hat dabei für die 
erste Person Plural ebenfalls verschiedene Referenzmöglichkeiten identifizieren können. Das inklusive WIR wurde in ihrem Korpus, wie auch in den anderen Studien bereits gezeigt, vor allem dazu benutzt, die Zugehörigkeit des Autors zu verschiedenen Gruppierungen hervorzuheben. Eine der wichtigsten Funktionen erfüllt dabei die WIR-Variante, die Kresta und Steinhoff als „Fachkreis-WIR“ bezeichnet haben. Hier demonstriert der Autor seine Mitgliedschaft in der konkreten wissenschaftlichen Community, für die er den Text schreibt. Diesen Gebrauch von WIR interpretiert Sanderson dahingehend, dass die Autoren so ihre Identität als Wissenschaftler und Forscher hervorheben und stärken, indem sie sich selbst gezielt als Teil einer anerkannten wissenschaftlichen Gemeinschaft darstellen und sich in dieser Gemeinschaft und auch in Bezug zu anderen Wissenschaftlern positionieren. Dies ist nach Sanderson ein wichtiges Hilfsmittel, um die eigenen Aussagen und Hypothesen zu stärken und sie damit zu legitimieren. Darüber hinaus wird durch dieses inklusive WIR-als-Wissenschaftlerund-Forscher die Beziehung zum Leser gestärkt, da die gemeinsame Zugehörigkeit zu der gleichen Gruppe betont wird (Sanderson|2008a: 146).

Eine weitere Referenz des inklusiven WIR, die insbesondere in Sandersons deutschsprachigen linguistischen Texten vorkam, ist der Bezug zur einer gemeinsamen ethnolinguistischen Gruppe, was als Unterkategorie des oben beschriebenen „GemeinschaftsWIR" gewertet werden kann. Diese Strategie dient nach Sanderson vorrangig dazu, Solidarität mit dem Leser herzustellen, von dem angenommen wird, dass er den gleichen kulturellen und sprachlichen Hintergrund hat wie der Autor. Wenn dem so ist, kann dieses WIR die Gemeinsamkeiten zwischen Autor und Leser hervorheben und deren Beziehung stärken. Allerdings kann diese Strategie auch ins Gegenteil umschlagen, wenn nämlich der Leser nicht der gleichen ethno-linguistischen Gruppe angehört wie der Autor (Sanderson 2008a: 147). In diesem Fall schafft dieser WIR-Gebrauch Sanderson zufolge eher eine Distanz zwischen dem Autor und dem Leser. Dass sie diese Strategie nur in deutschprachigen Texten nachweisen konnte, interpretiert Sanderson so, dass die englischsprachigen Autoren eher als die deutschen für ein internationales Publikum schreiben (Sanderson|2008a: 147).

Eine weitere Gruppe, zu der das inklusive WIR Zugehörigkeit konstruieren kann, ist eine spezifische Forschungsgruppe. Dies wird überwiegend in Danksagungen realisiert und wird ebenfalls nur in Sandersons deutschen Texten verwendet, was ihrer Meinung nach mit den Förderungsstrukturen der deutschen Geisteswissenschaften erklärt werden kann. In Deutschland sei es im Vergleich zu Großbritannien und den USA eher üblich, Forschungsgruppen anstelle von einzelnen Forschern zu fördern. Daher bestehe für deutsche Forscher auch eher die Notwendigkeit, ihre Zugehörigkeit zu einer möglichst prestigeträchtig geförderten Forschungsgruppe zu demonstrieren und dadurch implizit ihre wissenschaftliche Autorität zu steigern (Sanderson 2008a: 148). Darüber hinaus kann das Verschieben der Verantwortung für die im Text gemachten 
Aussagen weg von dem Autor als Einzelperson diese Aussagen stärken, da sie bereits im Vorfeld von einer größeren Gruppe validiert wurden (Sanderson 2008a: 148).

Weitere kleinere Gruppen, die Sanderson auf spezifische disziplinäre Kontexte zurückführt, sind das „WIR als Lehrende“, was ausschließlich in didaktisch orientierten Texten ihres Korpus verwendet wurde (Sanderson 2008a 148), und das „Philosophische WIR", das den Autor als Teil der Spezies Mensch identifiziert (Sanderson 2008a 150). Während das erste vor allem dazu dient, eine kollegiale Atmosphäre herzustellen und zu demonstrieren, dass der Autor auch über Lehrerfahrung verfügt, führt Sanderson das andere WIR auf die fachlichen Besonderheiten und Erfordernisse philosophischer Artikel zurück, in denen, gerade im deutschsprachigen Bereich, häufig mit Blick auf die Natur der menschlichen Wahrnehmung oder des menschlichen Denkens an sich argumentiert werde (Sanderson 2008a: 150).

Diese Charakterisierung des WIR-Gebrauchs, vor allem im Vergleich mit den weiter oben angeführten Kategorisierungen, macht noch einmal deutlich, wie stark das WIR in seiner Referenz variieren kann und dass es immer auch vom Kontext und vor allem vom Leser abhängt, wie er die jeweiligen Vorkommen interpretiert. Diese Ambiguität lässt vermuten, dass es in vielen Fällen sehr schwer ist, die Bedeutung des WIR im Text eindeutig zu ermitteln. Tatsächlich werden von allen Autoren eine Anzahl von Items als nicht eindeutig kategorisierbar eingestuft. Dies könnte dazu verleiten, anzunehmen, dass auch die Autoren nicht immer genau wissen, welche Referenz die von ihnen benutzten WIR haben (Sanderson 2008a 149). Sanderson betont jedoch in ihrer Analyse, dass sie davon ausgeht, dass die grundsätzliche Vagheit, die mit dem Gebrauch der ersten Person Plural einhergeht, von den Autoren ihres Korpus sehr gezielt und bewusst verwendet wird, um jeweils diejenige Zugehörigkeitsfacette hervorzuheben, die ihre Bedürfnisse (z. B. das Betonen von Solidarität mit dem Leser oder der Mitgliedschaft in einer bestimmten Gruppe, um die Glaubwürdigkeit und Verlässlichkeit ihrer Aussagen zu stärken) am besten umsetzen kann (Sanderson 2008a: 150):

I would question whether the switching is really indiscriminate, though it may initially seem so because of its complexity. Rather, the alternation of different, we/wir' memberships in quick succession shows academic authors distinguishing between the many different identities they concurrently occupy. In fact [...] the opposite appears to be the case: each type of membership claim serves a specific purpose within the text, and authors appear absolutely aware of this. (Sanderson 2008a: 149)

Unterschiedliche Gebrauchs- und Interpretationsmöglichkeiten des WIR haben auch Fløttum u. a. (2006) in ihrer Untersuchung englischer, französischer und norwegischer Fachartikel gefunden. Dabei haben sie sich aufgrund der Zusammensetzung ihres Korpus hauptsächlich auf den Gebrauch der Pluralformen in Artikeln, die von mehreren 
Autoren geschrieben wurden, konzentriert. Grundsätzlich haben sie aber auch Verwendungen beschrieben, die genauso in Artikeln von Einzelautoren zum Tragen kommen.

Auch sie unterscheiden dabei zwei Aspekte: Zunächst stellen auch sie die Frage nach der Referenz des Pronomens. Hier schwankt die Bedeutung vor allem zwischen einer praktischen Synonymität mit dem ICH, also dem alleinigen Bezug auf den Autor, und einer weitgefassten Bedeutung, die sich an das unpersönliche MAN annähert, also eine wie auch immer geartete Allgemeinheit darstellt. Daneben haben sie aber ebenfalls Bedeutungen der ersten Person Plural ermittelt, die sich am ehesten mit einem Bezug auf den Leser, d.h. als DU interpretieren lassen (Fløttum u. a. 2006. 95). Hier wird nochmals deutlich, dass die Bedeutung dieses Pronomens immer stark vom Kontext und der Interpretation des Hörers/Lesers abhängig ist.

Nach der grundlegenden Unterscheidung zwischen dem inklusiven und dem exklusiven Gebrauch des Autoren-WIR haben sie dann auf Grundlage ihrer Daten weitere Gebrauchsformen identifiziert, die sie als figurativ bzw. metonymisch werten (Fløttum u. a. 2006 98), nämlich das inclusive we for you, inclusive we for I und exclusive we for $I$. Dabei erfüllen diese drei verschiedenen metonymischen Formen sehr ähnliche rhetorische Funktionen. Das inclusive we for you betone vor allem die Kooperation zwischen Autor und Leser und erzeuge den Eindruck eines gemeinsamen Standpunktes, von dem aus die Informationen gemeinsam betrachtet werden (Fløttum u. a. 2006. 99). Das inclusive we for I deuten sie ähnlich und sprechen ihm ebenfalls eine verbindende Funktion zu, die die "togetherness“ (Fløttum u.a. 2006 100) von Autor und Leser betont. Dies habe auch den zusätzlichen Effekt, dass die Verantwortung des Autors durch diese "gefühlte“ Kooperation verringert werde. Und Ähnliches kann auch für das exclusive we for I, das dem authorial we entspricht, angenommen werden.

Diese Interpretation als metonymischer Gebrauch veranschaulicht also noch einmal, dass neben der Kernbedeutung des WIR („Ich und eine oder mehr andere Personen“) noch eine zweite Bedeutung mitschwingt. Dabei gehen Fløttum u. a. (2006: 99) davon aus, dass diese beiden Bedeutungen auch gleichzeitig vom Leser wahrgenommen werden und zu einem kognitiven „Blend“ verschmelzen. Diese Verschmelzung beider Referenzen, sei sie bewusst oder unbewusst, führe dann auch immer zu einer Beeinflussung des Lesers auf emotionaler Ebene (Fløttum u. a. 2006: 99).

Zusammenfassend lässt sich sagen, dass das WIR in verschiedene Unterkategorien aufgeteilt werden muss, abhängig davon, auf welche Personen(kreise) es sich bezieht (s. Abb. 3.2). Dabei ist zunächst zu entscheiden, ob die Referenz inklusiv oder exklusiv ist, also ob der Hörer/Leser mit einbezogen ist oder nicht. Das inklusive WIR wiederum kann in weitere Kategorien eingeteilt werden, abhängig davon, wie viele Personen bzw. welche Personenkreise neben dem Sprecher bzw. Autor einbezogen sind. Es kann also im am engsten gefassten Sinne nur den Autor und den Leser umfassen, es kann aber weiterreichend auch eine bestimmte Gemeinschaft mit unterschiedlichem 
Ausmaß bezeichnen. Diese Gemeinschaft kann von der Gruppe Wissenschaftler, die sich mit einem bestimmten Thema beschäftigt, über alle diejenigen, die mit einer bestimmten Methode arbeiten, bis hin zur kompletten scientific community reichen. Die benannte Gemeinschaft kann aber auch noch weiter reichen und letztlich alle Mitglieder einer bestimmten Kultur, einer Sprachgemeinschaft, einer Nationalität oder sogar die gesamte Menschheit umfassen.

Es wird also deutlich, dass es sich bei diesen Einteilungen nur um heuristische Größen handeln kann und dass die Abgrenzung bzw. Einordnung im konkreten Fall nicht immer ganz einfach verlaufen kann (vgl. auch Kresta 1995: 299). Bezogen auf das hier verwendete Korpus dürfte die Abgrenzung zwischen z. B. Soziologen als „engerem“ und Sozialwissenschaftlern als „weiterem“ Fachkreis in vielen Fällen schwer fallen.

Und die Unterscheidung zwischen WIR als Mitglieder einer Sprachgemeinschaft, einer kulturellen Gruppe, einer biologischen Spezies etc. ist sicherlich einerseits schwierig und andererseits wäre die Unterteilung des Gemeinschafts-WIR in alle diese und noch mehr Unterkategorien als Operationalisierung für eine empirische Untersuchung zu differenziert. Nichtsdestoweniger ist beim Gebrauch dieser Kategorien immer zu berücksichtigen, dass sie in sich immer noch eine Heterogenität vertreten, die bei Bedarf weiter differenziert werden kann und muss. In Abbildung 3.2 werden die für diese Arbeit relevanten Bedeutungsvarianten dargestellt.

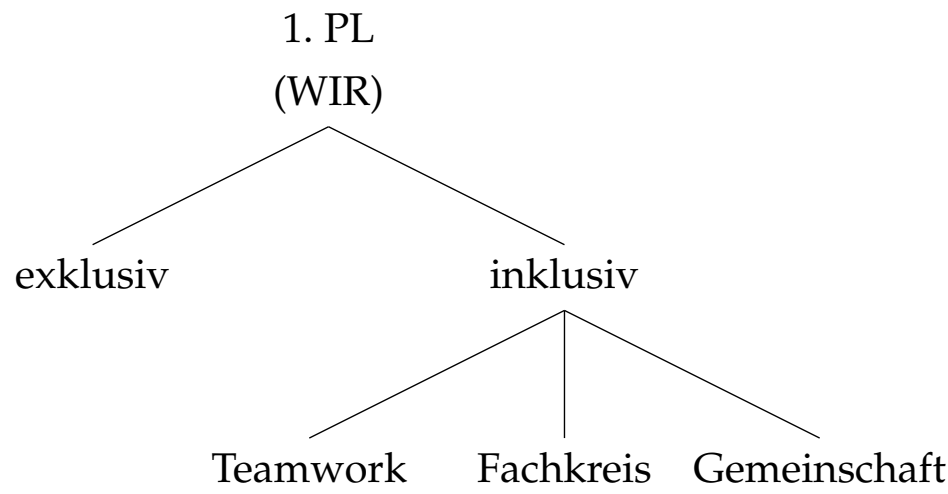

Abbildung 3.2: Bedeutungvarianten der 1. Person Plural

Das WIR dient also zunächst dazu, die Zugehörigkeit zu einer bestimmten Gruppe hervorzuheben: „wir Mitglieder einer Disziplin“, „,wir, Autor und Leser“, „,wir als Angehörige einer (Sprach-)Kultur" , "wir Mitglieder einer Forschungsgruppe“, ,"wir Menschen" etc.

By claiming membership in this way, the [...] authors draw attention to and reinforce their identity as researchers, positioning themselves as part of a traditionally-recognised discipline, and allying themselves with a respected social role as scholars. Foregrounding their membership of a particular disciplinary group can be a powerful means for authors to legitimise 
their opinions and lend their hypotheses credibility. [...] Using the inclusive we , we' to mean ,we researchers' also strengthens the relationship with the imagined reader, by emphasising shared membership of a particular discourse community. (Sanderson 2008b: 83)

Dabei wechseln die Autoren häufig zwischen diesen verschiedenen Zugehörigkeiten und Identitäten (was auf eine bewusste und strategische Wahl von Identität hindeutet) und der Leser muss aus dem Kontext erschließen, welche Gruppe gemeint ist. Dies ist nicht immer eindeutig möglich und diese Ambiguität kann als Stilmittel zu unterschiedlichen Zwecken eingesetzt werden. Einerseits kann das WIR statt dem ICH Bescheidenheit ausdrücken. Über das WIR kann sich der Autor mit dem Leser auf eine Stufe stellen und den Eindruck erwecken, dass beide gemeinsam zu den im Text dargestellten Erkenntnissen gelangen, die Daten gemeinsam interpretieren usw. Er kann sich in den Hintergrund stellen und der Gefahr entgehen, seine Meinung zu sehr in den Vordergrund zu spielen.

Andererseits kann durch das WIR aber die geäußerte Meinung auch umfassender und eher allgemeingültig wirken, als wenn sie über ein ICH als Subjekt bzw. Agens realisiert würde. Gelegentlich kann das WIR dem Autor sogar dazu dienen, die eigene Wichtigkeit hervorzuheben (Sanderson|2008b: 87). Grundsätzlich bewirkt das WIR im Gegensatz zum ICH jedoch eine Distanzierung, der Text wird unpersönlicher und die Person des Autors tritt in den Hintergrund (vgl. Sanderson 2008b: 87), was auch Kuo (1999) hervorhebt:

The fact that the writer of a single-authored article uses we, instead of I, as he/she is referring to himself/herself, may suggest an intention to reduce personal attributions. (Kuo|1999. 125)

\subsubsection{Unpersönliches MAN}

Das letzte Pronomen, das in dieser Untersuchung nur ergänzend zu den Pronomen der ersten Person betrachtet werden soll, ist das unpersönliche MAN 16 Dieses war ebenfalls schon mehrfach Gegenstand von Untersuchungen zum wissenschaftlichen Schreiben, besonders im Vergleich mit dem ICH und dem WIR. Obwohl das MAN zumindest in seiner grundlegenden denotativen Bedeutung keine Ausprägung der ersten, sondern der dritten Person ist, macht es Sinn, es hier in ausgewählten Funktionen mit zu betrachten. Wie im Folgenden ausgeführt wird, gibt es Vorkommen des MAN, die eindeutig als verfasserreferenziell gewertet werden müssen.

\footnotetext{
${ }^{16}$ Zwar sind das deutsche MAN und das englische ONE in ihrer Bedeutung nicht immer eindeutig deckungsgleich, ich schließe mich jedoch den hier zitierten Arbeiten an, die es als ausreichend vergleichbar behandelt haben. Im Folgenden wird die Bezeichung MAN verwendet, wenn das Pronomen an sich gemeint ist; es steht also sowohl für das deutsche MAN als auch für das englische ONE.
} 
Darüber hinaus unterscheiden sich das deutsche und das englische Teilkorpus in Hinblick auf die Verwendungshäufigkeit der ersten Person Singular so deutlich, dass es sich anbietet, das unpersönliche Pronomen als zusätzliche Vergleichsdimension mit einzubeziehen. Diesen Ansatz haben auch Fløttum u. a. (2006) verfolgt:

Like many others we claim that first person pronouns represent the most explicit author presence, whatever the discoursal genre is. However, in our studies of author presence in research articles, we realised already in a pilot study [...] that the differences between English articles, on the one hand, and French articles, on the other, are so great that we found it necessary to investigate the extent to which other possible personal constructions are used in French. (Fløttum u. a. 2006, 110)

Zwar wird das englische ONE im Vergleich zu anderen Sprachen deutlich weniger benutzt (Fløttum u.a. 2006: 111), Fløttum u.a. (2006) gehen aber mit Johansson (2002) trotzdem davon aus, dass das englische ONE und das deutsche MAN (sowie auch das norwegische MAN) in Bezug auf ihre möglichen Referenzen vergleichbar sind, auch wenn es offenbar deutliche Unterschiede in ihrem Gebrauch in wissenschaftlichen Texten gibt, so das es durchaus sinnvoll ist, sie zu vergleichen.

Auch Matthias Hutz hat in Bezug auf die unpersönlichen Pronomen MAN und ONE deutliche Unterschiede zeigen können. Während er in seinem deutschsprachigen Korpus bei 1,2 \% der finiten Verbformen ein MAN als Subjekt gefunden hat, was in seinem Korpus häufiger ist als die erste Person Singular und Plural zusammengenommen, kam das unpersönliche ONE in seinem englischsprachigen Teilkorpus so gut wie nicht vor (0,1 \%) (Hutz 1997: 238). Ähnliche Ergebnisse haben auch andere Untersuchungen, die das Deutsche und das Englische (Sanderson 2008a) oder auch das Englische und andere Sprachen (Fløttum u.a. 2006) verglichen haben, gezeigt. ${ }^{17}$

English one is rather infrequent. It is generally considered as formal, even pretentious and affected, and it appears that many people avoid using it altogether [...]. (Fløttum u.a. 2006; 68)

Das unpersönliche MAN steht für einen „generalisierten Aktanten“, der unterschiedliche Referenten widerspiegeln kann (Steinhoff 2007, 227). Diese Vagheit in der Referenz reicht noch deutlich weiter als beim WIR und führt dazu, dass die Aussagen, die mit einem unpersönlichen Subjekt getroffen werden, häufig eine weiterreichende intersubjektive Gültigkeit suggerieren als Aussagen mit personalisierten Subjekten (Steinhoff 2007. 227). Dies ist gerade in wissenschaftlichen Texten von Vorteil, wenn der Autor Aussagen oder Erkenntnisse, zu denen er subjektiv als Handelnder gelangt ist, als

\footnotetext{
${ }^{17} \mathrm{Im}$ hier untersuchten Korpus wird das ONE jedoch tatsächlich gelegentlich gebraucht und kommt mit einer absoluten Anzahl von 120 sogar auf einen höheren Wert als das deutsche ICH (105). Die Auswertung dieser Werte erfolgt in Abschnitt 5.1.3 und Abschnitt5.1.3.3.
} 
möglichst allgemeingültig darstellen will und sich selber bewusst nicht als diejenige Instanz einbringen will, die für diese Aussagen, Erkenntnisse etc. verantwortlich ist.

Since the reference of these pronouns typically has to be determined by the co(n)text, they represent a very specific means for hiding as well as for bringing forth different voices. On the one hand, we can say that the pronoun is very useful to authors who want to hide; on the other, it is useful to authors who want to bring in the voice of a larger community, without having to be specific about the extension of this community. (Fløttum u. a.|2006: 111)

Insofern stellt sich also die Frage, welche referenzielle Bedeutung die unpersönlichen Pronomen in wissenschaftlichen Artikeln annehmen können. Hier bietet sich der Vergleich mit dem Gebrauch von WIR an.

Kuo beispielsweise hat zwei unterschiedliche Diskursfunktionen für das MAN identifiziert. Es könne ihrer Meinung nach erstens als Referenz auf die Forscher bzw. Wissenschaftler im Allgemeinen dienen und damit das geteilte Interesse, Ziel oder Wissen hervorheben. Wenn sich die Referenz dagegen nur auf den Autor oder aber den Autor und den Leser bezieht, diene dies dazu, eine Meinung oder ein "action statement" zu äußern (Kuo 1999. 129). Grundsätzlich habe es aber immer einen distanzierenden und damit verallgemeinernden Effekt.

One, instead of we or I, can make the opinion less personal and suggest that the opinion is widely held, or the action would be taken by any researcher in a given situation. (Kuo 1999. 129)

Kresta (1995) und nach ihm Steinhoff (2007, 208) unterteilen die verschiedenen MAN, ebenso wie das WIR, in das Autoren-MAN, das Fachkreis-MAN und das Gemeinschafts-MAN. Dabei fassen sie unter das Autoren-MAN diejenigen Vorkommen, bei denen die Handlung an den Autor als ausführende Person gebunden ist. Das AutorenMAN konnte Kresta primär in Rezensionen nachweisen (Kresta 1995. 77). Ansonsten hat Kresta (1995) in seinem Korpus vor allem Vorkommen des Fachkreis-MAN gefunden (Kresta 1995 302), sie machen ca. drei Viertel der von ihm gefundenen MANVorkommen und sogar $80 \%$ der ONE-Vorkommen aus. In dem Teilkorpus wissenschaftlicher Aufsätze konnte sogar über $90 \%$ beider Pronomen diese Referenz zugeschrieben werden (Kresta 1995, 303). Dabei wurde das Fachkreis-MAN überwiegend in metaligualen Handlungen verwendet (Kresta 1995: 319). Das Gemeinschafts-MAN dagegen machte nur etwa $10 \%$ der Items aus, jedoch wurde das ONE in Krestas Texten mit dieser Bedeutung prozentual häufiger benutzt als das MAN (Kresta|1995: 312). Grundsätzlich wurde aber das MAN in seinen Texten deutlich mehr verwendet als das ONE (Kresta|1995: 303).

Dass das MAN im Deutschen eine bedeutende Rolle spielt, haben auch Steinhoffs Analysen gezeigt. Im Vergleich zu den Pronomen der zweiten Person hat Steinhoff 
gezeigt, dass das MAN in den von ihm untersuchten Expertentexten mehr als dreimal so häufig verwendet wurde wie das ICH und 2,5 mal so häufig wie das WIR (Steinhoff) 2007. 210).

Steinhoff hat in seiner Untersuchung vor allem die Kombination des unpersönlichen Pronomens mit den Modalverben beobachtet und analysiert. Dabei hat er herausgefunden, dass das MAN am häufigsten mit können kombiniert wird, was für $57 \%$ der MAN-Vorkommen in seinem Korpus der Fall ist. Danach wird das MAN mit müssen $(14 \%)$, sollen (12\%), wollen (10\%), dürfen (4\%) und mögen (2\%) benutzt (Steinhoff 2007. 228). Steinhoff weist jedoch darauf hin, dass die Kategorisierung verschiedener MAN-Typen nur idealtypisch sein kann und sich nicht zur Beschreibung des tatsächlichen MAN-Gebrauchs in seinem Korpus eigne, da die Belege größtenteils nicht eindeutig bestimmbar seien (Steinhoff|2007 208). Dies wurde auch für das hier untersuchte Korpus festgestellt, sodass in der folgenden Auswertung das MAN nur dahingehend betrachtet wird, wie es in Handlungen benutzt wird, die auch mit dem ICH und dem WIR realisiert werden (könnten). Andere Gebrauchsweisen, unklare Formen und Kombinationen mit Modalverben, die die Handlungsbedeutung des Vollverbs modifizieren und damit eine eindeutige Zuordnung verhindern, werden aus diesem Grund hier nicht berücksichtigt. Ebenfalls nicht weiter thematisiert werden die Funktionen des MAN als Ersatz für die zweite Person (s. z. B. Sanderson 2008a: 113) und in Kombination mit Imperativen (Kresta|1995: 126).

\subsubsection{Autorenrollen}

Die oben dargestellten Untersuchungen beschreiben den Gebrauch der Pronomen aus einer überwiegend textlinguistischen Perspektive und erklären die unterschiedlichen Gebrauchsweisen mit unterschiedlichen Textkonventionen. Hier wird jedoch ein etwas anderer Fokus gesetzt. Der ICH-Gebrauch bzw. seine Vermeidung und Ersetzung durch andere Pronomen werden als Mittel der Identitätskonstruktion gesehen (vgl. auch Sanderson 2008a). Diese Perspektive ist dynamischer und macht deutlich, dass die Entscheidungen, die dem Gebrauch der verschiedenen Pronomen zugrunde liegen, nicht notwendigerweise nur auf textueller oder linguistischer Ebene, d. h. in der Textsorte „Wissenschaftlicher Artikel“ zu suchen sind, sondern vielmehr (auch) auf den sozialen und kulturellen Gegebenheiten fußen, unter denen der Text produziert wird. Daneben sind diese Entscheidungen aber auch abhängig von individuellen Präferenzen des Autors sowie anderen (sozialen) Eigenschaften wie dem akademischen Status oder Schreib- und auch anderen Erfahrungen des jeweiligen Autors (vgl. z. B. Ivanič 1998). Entsprechend ist diese Untersuchung sozio- bzw. interkulturell-linguistisch ausgerichtet, $d$.h. es werden nicht nur linguistische, sondern auch soziokulturelle Regeln angenommen, die dem Gebrauch der Pronomen zugrunde liegen und sich damit im Sprachgebrauch äußern. Konkret geht es hier also darum, wie die Identität „,kompe- 
tenter Wissenschaftler" bzw. „kompetenter wissenschaftlicher Autor" sprachlich konstruiert und kommuniziert wird.

Identitäten werden kommuniziert, indem die Aktanten Rollenslots ausfüllen und unterschiedliche Rollen übernehmen (s. Abschnitt 2.2.1). Diese Rollen wiederum werden anhand von Handlungen umgesetzt. In dieser Untersuchung geht es entsprechend darum, welche Handlungen sprachlich dargestellt und über die Pronomen der ersten Person, insbesondere im Singular, der Person des Autors zugeschrieben werden.

Aufgrund der textlinguistischen Perspektive bisheriger Untersuchungen liegt dort häufig der Fokus auf den Text- oder Diskursfunktionen, die die Pronomen bzw. die Sätze, in denen sie vorkommen, erfüllen. Diese Funktionen können in den meisten Fällen auch als Sprechhandlungen interpretiert werden, wobei, wie auch bei Diskursfunktionen (vgl. z. B. Hyland 2002a), der Fokus eben auf der Funktion liegt, die die jeweilige Handlung im Text erfüllt. Hier jedoch wird das sprachlich dargestellte Handeln betrachtet und dahingehend interpretiert, welche Rolle der Autor sich damit außerhalb des Textes bzw. über den Text hinauswirkend zuschreibt. Diese sprachlich dargestellten Handlungen werden von den im jeweiligen Satz verwendeten Verben bzw. Verbkomplexen, $\mathrm{d}$. h. von der denotativen semantisch-pragmatischen Bedeutung des Verbs, abgeleitet (vgl. Fløttum u. a. 2006: 6). Die Handlung „Daten erheben“ kann beispielsweise durch die Verben interviewen, befragen, sammeln, beobachten etc. dargestellt werden. Entsprechend lässt sich ermitteln, welche Handlungen von der Community für einen kompetenten Wissenschaftler und Kollegen akzeptiert werden.

Im Folgenden werden nun die Kategorisierungen aus vorhergehenden Untersuchungen, die den Gebrauch von Pronomen der ersten Person aus der Perspektive der Umsetzung (diskursiver) Rollen analysiert haben, dargestellt. Darauf aufbauend wird das Kategorisierungsschema beschrieben, das der hier vorgestellten Analyse zugrunde liegt. In dieses Schema sind neben den in diesem Kapitel vorgestellten auch weiterführend Ergebnisse aus Arbeiten eingegangen, denen kein explizit rollenorientierter Ansatz zugrunde lag (s. Tabellen 3.1 und 3.2 auf S. 84 bzw. 85 ).

\subsubsection{Autorenidentitäten nach Tang und John (1999)}

Eine der frühesten und auch am deutlichsten konturierte Kategorisierung von Identitäten und Rollen im wissenschaftlichen Schreiben haben Ramona Tang und Suganthi John durchgeführt (Tang und John 1999) ${ }^{18}$. Dabei gehen sie von drei verschiedenen Rollentypen aus, die abhängig sind von der Reichweite, die diese Rollen im sozialen Leben haben können. Sie unterscheiden entsprechend zwischen Rollen, die auf der sozialen, der Diskurs- oder der Genre-Ebene vollführt werden können (vgl. auch Gerhardt (2008) und die Diskussion in Abschnitt 2.2.2). Diese Rollen können jedoch

\footnotetext{
${ }^{18}$ Sie haben in ihrer Untersuchung bzw. deren Darstellung in Tang und John (1999) die Ergebnisse zur ersten Person Singular und Plural nicht getrennt, daher wird diese Studie hier diskutiert und nicht im vorangegangenen Teil.
} 
alle textuell in einem wissenschaftlichem Artikel umgesetzt werden (Tang und John 1999: 25).

In ihrer Untersuchung stellten sie die Frage danach, wie die Pronomen der ersten Person in akademischen (studentischen) Texten verwendet werden, um die Identität des Autors zu konstruieren. Da diese Untersuchung auf studentischen Texten basiert, sind die Ergebnisse und vor allem deren Interpretationen nicht direkt auf die Untersuchung wissenschaftlicher Texte übertragbar ${ }^{19}$. Dennoch ist anzunehmen bzw. zumindest nicht auszuschließen, dass diese Sichtweisen auch bei anonymen ReviewProzessen eine Rolle spielen, dass also englischsprachig sozialisierte Reviewer vergleichbare Assoziationen haben, wenn sie anonyme Texte lesen, in denen die erste Person nicht oder nur in wenig autoritären Rollen benutzt werden. ${ }^{20}$

Basierend auf den Ergebnissen von Roz Ivanič (1998) sowie der Untersuchung ihres eigenen Korpus aus publizierten Text(teil)en haben Tang und John eine Typologie von sechs Autorenrollen entwickelt, die alle durch den Gebrauch der ersten Person Singular realisiert werden. Dabei haben sie sowohl die erste Person Singular als auch Plural und alle Kasus berücksichtigt. An ihrem Korpus aus 27 Essays, geschrieben von Erstsemestern an der Nationalen Universität Singapur, und Textproben aus publizierten Artikeln und Büchern aus dem Fach Linguistik konnten sie nachweisen, dass die erste Person in akademischen Texten der Linguistik tatsächlich unterschiedliche Funktionen erfüllt (s. Tang und John 1999: 26). Diese haben sie in sechs Autorenrollen zusammengefasst:

'I' as the

- representative

- guide through the essay

- architect of the essay

- recounter of the research process

- opinion-holder

- originator (Tang und John|1999: 27-29)

Angelehnt an Ivanič (1998) interpretieren sie diese sechs Rollen anhand eines Kontinuums zunehmender Machtausübung (vergleichbar mit Hylands Kategorisierung von Autorenrollen nach Risiko (Hyland|2002a)).

By a powerful authorial presence, we mean that the writer displays a high level of authority within the text, where 'authority' has elements of both its common meanings of 'a right to control or command others' and 'knowledge or expertise in a particular field', as well as a meaning more specific

\footnotetext{
${ }^{19}$ Die Rollenkategorien, mit denen Tang und John gearbeitet haben, basieren jedoch auf der Analyse wissenschaftlicher Texte und sind daher auch direkt für diese Untersuchung relevant (Tang und John 1999: 26).

${ }^{20}$ Darauf deuten auch die Experteninterviews von Hyland (2001b) und Hyland (2002a) hin, weiterführend s. z. B. die Studie von Tardy und Matsuda (2009).
} 
to this article - the quality belonging to an 'author', where 'author' is used in [the] very specialized sense of 'a maker of meaning'. (Tang und John 1999. 26)

Die Repräsentantenrolle ist diejenige, die die Studierenden, deren Texte untersucht wurden, am häufigsten mit einem Pronomen der ersten Person umgesetzt haben. Diese Rolle ist dadurch charakterisiert, dass hier die erste Person in ihrer generischen Bedeutung und entsprechend ausschließlich im Plural verwendet wird ${ }^{21}$. Der Autor bezieht sich und ggf. den Leser in eine größere Gruppe von Menschen ein. Dies interpretieren Tang und John so, dass er sich damit praktisch zu einer "non-identity" macht (Tang und John 1999. 27), da der Leser trotz der grammatischen Selbstreferenz nichts über den Autor erfährt. Dadurch ist diese Rolle die am wenigsten machtvolle im o. g. Sinne.

Insgesamt haben Tang und John über $40 \%$ (39 von insgesamt 92) aller Vorkommnisse in ihrem studentischen Korpus als representative kategorisiert (Tang und John 1999 30). Dies erklären sie damit, dass Studierende sich gegenüber ihren Lehrenden in einer schwächeren Position befinden und sich selber daher nicht zugestehen, eine autoritäre bzw. authoritative Rolle einzunehmen und sich damit als Mitglied der wissenschaftlichen Gemeinschaft zu positionieren (Tang und John 1999. 30). Allerdings sehen sie darin auch ein wichtiges Anzeichen dafür, dass die Studierenden versuchen, zumindest über den Gebrauch gemeinsamen und etablierten Wissens praktisch den ersten Zugang in die Gemeinschaft zu erhalten.

Ein wenig autoritärer im obigen Sinne ist der Autor als guide. In dieser Funktion, die ebenfalls pluralisch umgesetzt wird, nimmt der Autor den Leser praktisch mit auf eine "Reise“ durch den Artikel. Tang und John benutzen in diesem Zusammenhang die Metapher eines "fremden Landes“ (foreign country (Tang und John 1999 27)), das der Leser mit dem Autor als Reiseführer erkundet. Dem Autor kommt dabei die Aufgabe $z u$, dem Leser den richtigen Weg zu weisen, ihn auf bestimmte wichtige Dinge hinzuweisen, ihn in Zeit und Raum zu verorten und letztlich am Ziel, d.h. an den vom Autor vertretenen Schlussfolgerungen, anzukommen. Dazu verwenden die Autoren in dem von Tang und John untersuchten Korpus vorrangig Verben der visuellen Wahrnehmung wie see, note oder observe (Tang und John 1999. 27).

Diese Rolle wird von knapp 34 \% (absolut 31) der in ihrem Korpus vorhandenen Pronomen der ersten Person realisiert, allerdings ebenfalls ausschließlich im Plural, was Tang und John als eine "safety in numbers-Mentalität" interpretieren (Tang und John 1999 32). Darüber hinaus stehe der Guide praktisch außerhalb des Textes und trage daher keine Verantwortung für das, was im Text gesagt wird, wodurch sich der (studentische) Autor deutlich weniger den möglichen „Attacken“ der Leser bzw. seines Dozenten aussetzt als in den folgenden Rollen.

\footnotetext{
${ }^{21}$ vgl. die verschiedenen Formen des WIR, Abschnitt 3.3.1.2
} 
Das ' $I$ ' as the architect wird dagegen hauptsächlich als Singular realisiert und handelt entsprechend als Architekt des Textes, indem es den Text organisiert, strukturiert und den „roten Faden“ hervorhebt. Im Vergleich zum Guide übt der Architect in seinem Text ein wenig mehr Macht aus, da er nicht nur wahrnimmt, sondern seine aktive Einflussnahme auf den Text deutlich macht. Den Unterschied zum Guide definieren Tang und John folgendermaßen:

Where the architect has the responsibility of organizing and outlining the material in the writing (creating structure, as it were, in the writing process), there is a sense in which the guide merely seems to be showing the reader through an already existing terrain. (Tang und John 1999: 28)

Tang und John haben diese Rolle in ihrem studentischen Korpus nur 13 Mal mit der ersten Person realisiert gefunden. Stattdessen werden hier häufig andere Wege gesucht, diese notwendige Funktion im Text umzusetzen und Studierende weichen hier häufig aus, indem sie beispielsweise Passivkonstruktionen, Wendungen mit unbelebten Subjekten wie "this essay" oder Nominalisierungen bevorzugten. Tang und John werten dies als möglichen Hinweis darauf, dass die studentischen Autoren ihrer Korpustexte eine positivistische Perspektive auf die Wissenskonstruktion haben und daher versuchen, ihre Texte möglichst distanziert und unpersönlich klingen zu lassen.

Berichte über den Forschungsprozess und die angewandten Methoden charakterisieren den Autor als Recounter und stellen ihn überwiegend im Singular dar. Diese Rolle wird entsprechend besonders über sog. material process verbs wie work, read, interview oder collect (Tang und John (1999: 28) nach Halliday (1994)) und auch häufig in der Vergangenheit umgesetzt, da die beschriebenen Forschungshandlungen im Normalfall vor dem Verfassen des Textes stattgefunden haben und entsprechend dargestellt werden. Diese Rolle haben Tang und John vorrangig in denjenigen von ihnen untersuchten wissenschaftlichen Texten gefunden, denen eine empirische Forschung zugrunde lag. Da dies bei den studentischen Texten ihres Korpus nicht gegeben war, wurde diese Rolle auch nicht umgesetzt. Allerdings ist damit nicht ausgeschlossen, dass die Studierenden diese Rolle in der ersten Person umgesetzt hätten, wenn sie entsprechende Forschungshandlungen hätten dokumentieren wollen (Tang und John 1999: 33-34).

In der Rolle des Opinion-holder stellt der Autor seine Meinung bzw. Einstellung zu oder Perspektive auf bestimmte Zusammenhänge dar, bei denen es sich vorrangig um bereits etabliertes Wissen und Fakten handelt. Hier kann er beispielsweise Zustimmung oder Ablehnung signalisieren oder einfach sein Interesse äußern, was er durch die Darstellung mentaler bzw. kognitiver Prozesse wie z. B. think oder find ausdrückt (Tang und John 1999: 28). Dies wird natürlich nicht immer in der ersten Person (Singular) umgesetzt, aber wenn ja, dann übernimmt der Autor schon ein deutliches Maß an Verantwortung und Power. 
Die nach der Kategorisierung von Tang und John machtvollste Rolle ist jedoch die des Originator. In dieser Rolle stellt der Autor seinen eigenen Beitrag heraus und weist die Leser auf seinen originären neuen Beitrag zum Wissensbestand der Community hin. Indem sie dies in der ersten Person vollziehen, verbreiten die Autoren jedoch nicht nur ihre eigenen Ideen und Ansichten, sondern sprechen sich selber deutlich die grundlegende Fähigkeit und damit auch das Recht und die Position zu, innerhalb der Community neues Wissen zu kreieren (Tang und John 1999: 29). Dies ist wohl die stärkste und gleichzeitig auch die riskanteste Art und Weise, die ein wissenschaftlicher Autor nutzen kann, um sich selbst als kompetent und glaubwürdig darzustellen bzw. „in Szene zu setzen“.

Diese beiden letzten Rollen hängen nach Tang und John eng zusammen und sie sehen in ihren wissenschaftlichen Texten eine „natürliche Progression“ (Tang und John 1999. 34) vom Opinion-Holder zum Originator, also von der Bewertung der Behauptungen anderer Wissenschaftler zur Äußerung der eigenen Position. Auch in ihren studentischen Texten konnten sie diese Rollen mit der ersten Person umgesetzt finden, allerdings nur vereinzelt. Sie erklären dies so, dass diese beiden Rollen offenbar sehr anspruchsvoll seien, in dem Sinne, dass die Autoren damit direkt die Verantwortung für ihre eigenen Behauptungen übernehmen, also den „writer-as-thinker“ explizit in den Text einbringen und der Kritik der Leser aussetzen (Tang und John 1999. 34).

Gerade Studierende tun sich damit nach Ansicht von Tang und John tendenziell schwer, da sie durch die Übernahme dieser Rollen signalisieren, dass sie sich mit ihren Lehrenden und den Verfassern der von ihnen verwendeten Literatur auf eine Stufe stellen und deren Positionen ggf. sogar kritisieren. Hier sind Studierende, insbesondere Studienanfänger, sicherlich schnell verunsichert, was sie, wie Tang und John vermuten, veranlasst, sich hinter unpersönlichen Wendungen und scheinbarer Objektivität zu „verstecken“ (Tang und John 1999: 35).

\subsubsection{Rhetorische Funktionen - die KIAP-Studie}

In der norwegischen KIAP-Studie 22 haben Kjersti Fløttum, Trine Dahl und Torodd Kinn vier verschiedene rhetorische Autorenrollen identifiziert, anhand derer sie das explizite Auftreten der Autoren in ihren Texten kategorisieren. Dazu haben sie die Pronomen der ersten Person Singular isoliert und im Zusammenhang mit ihrem direkten Kotext untersucht. Dieser Kotext ist in erster Linie die verbale Konstruktion. Die semantisch-pragmatische Bedeutung des Verbs bzw. Verbkomplexes, mit dem das Pronomen kombiniert wird, definiert die rhetorische Rolle des Autors grundlegend. Darüber hinaus wurden auch metatextuelle Ausdrücke im Kotext betrachtet, die diese Bedeutung modifizierten (vgl. Fløttum u. a. 2006 82). Die rhetorischen Rollen werden dabei folgendermaßen kategorisiert (vgl. Fløttum u. a. 2006: 83-84):

\footnotetext{
${ }^{22}$ Für einen kurzen Überblick s. Kap. 3.3.1.1. S. 56
} 
1. Die writer role drückt einerseits Prozesse aus, die verbale oder graphische Darstellungen umfassen. Diese werden von Diskursverben wie describe, illustrate oder present umgesetzt. Daneben schließt die Definition dieser Rolle auch Prozesse ein, die direkt auf die Textstrukturierung und Lesersteuerung abzielen, wie z. B. begin by, move on to, (re)turn to etc.

2. Die researcher role wird realisiert von so genannten research verbs, die diejenigen Aktivitäten umfassen, die direkt auf den Forschungsprozess abzielen: analyse, assume, compare, explore, study, test etc.

3. Die arguer role wird von sog. position verbs ausgedrückt, die eine Positionierung und Darstellung des eigenen Standpunktes erlauben: argue, claim, dispute, reject etc.

4. Die evaluator role schließlich bringt die emotionale und bewertende Seite des Autors zum Ausdruck und wird von sogenannten evaluation und emotion verbs wie feel, be content of, be sceptical about etc. dargestellt.

Dabei war mit ca. 50 \% aller Fälle die researcher role die dominanteste Rolle, gefolgt von der writer role (ca. $25 \%$ ) und der arguer role mit ca. $14 \%$. Die evaluator role tritt nur selten und nur in einigen wenigen Artikeln auf (4\%), Fløttum u. a. (2006) betrachten sie daher auch eher als stilistisches Merkmal einzelner Texte, halten sie aber nicht für relevant für eine generelle Charakterisierung wissenschaftlicher Artikel. Neben diesen drei Rollen haben sie auch vereinzelt Kombinationen von Writer und Researcher (5\%) sowie Writer und Arguer (2 \%) gefunden (Fløttum u. a.|2006: 91).

Beim interlingualen Vergleich hat sich dann gezeigt, dass der englischsprachige wissenschaftliche Stil offenbar expliziter und direkter ist als der norwegische und der französische. Dieses Ergebnis ist konsistent mit Ergebnissen anderer vergleichender Studien (Fløttum u. a. 2006: 92). Disziplinspezifische Unterschiede zeigten sich vor allem daran, dass insbesondere die arguer role in linguistischen Texten häufiger benutzt wurde als in wirtschaftswissenschaftlichen Texten. Mediziner benutzten die erste Person Singular in allen Rollen deutlich seltener (Fløttum u. a. 2006: 92-93), was auch mit Hylands Ergebnissen zum Unterschied zwischen hard und soft disciplines korrespondiert (z. B. Hyland 2000, 2003, 2008) und weswegen sie die medizinischen Texte in ihrer Betrachtung der ersten Person nicht weiter berücksichtigt haben (Fløttum u.a. 2006: 92).

\subsubsection{Autorenrollen nach Steinhoff}

Torsten Steinhoffs Studie zur wissenschaftlichen Textkompetenz (Steinhoff 2007$)^{23}$, die sich u. a. auch mit dem ICH-Gebrauch in Texten von deutschen Wissenschaftlern und

\footnotetext{
${ }^{23}$ Für einen kurzen Überblick s. Kap. 3.3.1.1. S. 55
} 
Studierenden beschäftigt, stellt die aktuell umfassendste Untersuchung im deutschsprachigen Raum dar, die mit einem Rollenbegriff arbeitet.

Laut Schütz bringt sich das Individuum in einer Rolle in die theoretischwissenschaftliche Domäne ein - in der Rolle des „Theoretikers“. Ich möchte Schütz' These aufgreifen und zeigen, dass sich in Wissenschaftstexten verschiedene "Ich-Typen“ nachweisen lassen, die mit bestimmten Rollen des Schreibers zusammenhängen. (Steinhoff 2007; 174)

Basierend auf einer Analyse des jeweiligen syntagmatischen Kontextes hat Steinhoff drei Ich-Typen identifiziert, die bestimmte Rollen des Schreibers repräsentieren (Steinhoff 2007: 174) und durch die erste Person Singular realisiert werden: das Verfasser-Ich, das Forscher-Ich und das Erzähler-Ich (Steinhoff 2007: 180-204). Dabei hat er sich vorrangig am finiten Verb, d. h. der dem Agens zugewiesenen Handlung orientiert. Diese Handlung signalisiert in vielen Fällen die Rolle, die der Schreiber sich damit zuweist (Steinhoff|2007: 176-177).

Das Verfasser-Ich tritt v. a. in Passagen der Textkommentierung auf, d. h. in Wendungen, die der Lesersteuerung dienen. Weiterhin fasst Steinhoff darunter auch Textprozeduren, die Intertextualität herstellen (zitieren, verweisen etc.) sowie Danksagungen.

Das Forscher-Ich dient u. a. dazu, Originalität zu bezeugen. Es wird v. a. für Begriffsbildungen und Definitionen eingesetzt, aber auch um Hypothesen zu explizieren sowie vereinzelt auch, um sich kritisch über andere Autoren bzw. deren Annahmen zu äußern.

Das Erzähler-Ich dagegen wird nur von Studierenden in den frühen Phasen ihres Studiums verwendet. Es wird benutzt zur Auto-Narration, d.h. die Autoren erzählen von sich selbst und ihren Erfahrungen und Empfindungen, die sich v.a. auf die Hausarbeit beziehen. In den von Steinhoff durchgeführten Experteneinschätzungen, für die 14 Rater mit abgeschlossenem geisteswissenschaftlichem Hochschulstudium und ,jahrelanger Erfahrung mit wissenschaftlichen Texten“ (Steinhoff 2007: 158-159) anhand festgelegter Bewertungskriterien ihre Einschätzung zur Wissenschaftlichkeit der untersuchten Formulierungen gaben, wurde diese Form als eindeutig nicht wissenschaftlich eingestuft (Steinhoff 2007: 200-201).

Steinhoff hat festgestellt, dass es bestimmte Verben gibt, die bevorzugt mit ICH kombiniert werden (Steinhoff 2007, 177). Der Verfasser ist häufig das Subjekt für eingehen auf, darstellen, vorstellen und der Forscher wird oft mit denken, halten für, sprechen von kombiniert. Für den Erzähler konnte Steinhoff kein Verb als eindeutigen Indikator nachweisen.

Steinhoff hat in seiner Studie gezeigt, dass die erste Person Singular auch in deutschen Texten sowohl von Wissenschaftlern als auch von Studierenden benutzt wird. Unterschiede zwischen den von ihm untersuchten Disziplinen lassen sich dahingehend feststellen, dass das ICH am häufigsten in linguistischen Texten verwendet wird, 
weniger in literaturwissenschaftlichen und am seltensten in Texten der Geschichtswissenschaft. Aus der Perspektive der Schreibentwicklung sei nach Steinhoff auffällig, dass Studierende am Anfang ihres Studiums das ICH wesentlich häufiger und auch in anderen rhetorischen Zusammenhängen benutzten als wissenschaftliche Autoren. Im Laufe der fortschreitenden Sozialisation nähere sich in den fachspezifischen wissenschaftlichen Kontext der ICH-Gebrauch in studentischen Texten jedoch dem in wissenschaftlichen Artikeln an. Dabei würden auch die typischen disziplinspezifischen Unterschiede erkennbar, was vermutlich auf die fortschreitende fachwissenschaftliche Lese- und Schreibsozialisation und darauf, dass sich Schreibanfänger an den Texten ihres Faches orientieren, zurückgeführt werden könne (Steinhoff 2007; 173-174).

Insbesondere hat Steinhoff beobachtet, dass der ICH-Gebrauch, der sich in seinem Korpus vorrangig auf die Textteile Einleitung, Schluss und Fußnoten konzentriert, in den Textteilen Einleitung und Schluss im Lauf der Schreibsozialisation weniger wird, während das ICH in Fußnoten und auch im Hauptteil bei fortgeschrittenen Autoren häufiger auftritt als bei Schreibanfängern (Steinhoff 2007 179). Hierfür sieht er drei mögliche Erklärungen. Erstens könnte es sein, dass mit zunehmender Schreiberfahrung andere, vorrangig agenslose Verfasserreferenzen wie z. B. Passivkonstruktionen benutzt werden (Steinhoff 2007: 179). Zweitens verzichten geübtere Schreiber mehr und mehr auf autobiographische Äußerungen in ihren Texten, die in Hausarbeiten jüngerer Studierender noch häufiger vor allem in den Einleitungs- und Schlussteilen vorkommen. Und drittens würden Fußnoten im Verlauf der Schreibsozialisation zunehmend benutzt, um "metakommunikativen Begleittext" zu liefern, wohin dann die Selbstreferenzen „,ausgelagert“ werden können (Steinhoff 2007 180).

Der Gebrauch des ,ich' erfolgt also mit zunehmender Schreiberfahrung bewusster und kontrollierter. (Steinhoff 2007; 173)

Insgesamt hat Steinhoff nur eine „tendenzielle Vermeidung“ des ICH nachweisen können, allerdings haben sich sowohl in seinem studentischen als auch dem Expertenkorpus große Streuungswerte ergeben, in beiden Korpora kam in rund $40 \%$ der Texte kein ICH vor. Dabei haben die Studierenden das ICH jedoch insgesamt noch deutlich häufiger benutzt als die fortgeschrittenen Autoren, nämlich 2,34 Mal so oft.

\subsubsection{Diskursfunktionen nach Hyland}

Angelehnt an die Untersuchung von Tang und John (Tang und John 1999) und basierend auf der Auswertung seiner eigenen Korpora ${ }^{24}$ hat Ken Hyland fünf Diskursfunktionen identifiziert, in denen die erste Person benutzt wird, um die Rolle des Autors im jeweiligen Kontext zu kennzeichnen (Hyland 2002a: 1098-1106). Zusätzlich dazu hat er Interviews mit schreiberfahrenen Experten aus den von ihm untersuchten Disziplinen

\footnotetext{
${ }^{24}$ s. Kap. 3.3.1.1. S. 57
} 
durchgeführt, in denen er sie zu ihren eigenen Erfahrungen, Einschätzungen und Präferenzen beim Lesen und Schreiben wissenschaftlicher Texte in ihrer Disziplin befragt hat (Hyland 2001b: 211).

Die wesentlichen Diskursfunktionen, die sich in Hylands Analyse herausgestellt haben und die wissenschaftliche und studentische Autoren in der ersten Person ausführen, sind folgende:

1. Expressing self-benefits: Hier wird die erste Person verwendet, um über die persönlichen Erfahrungen, die der Autor während seines Projektes gemacht hat, zu berichten. In dieser Funktion wird die erste Person ausschließlich von studentischen Autoren verwendet. Da es um persönliche Eindrücke und Erfahrungen geht, setzt sich der Autor hier keiner Gefahr aus „etwas Falsches“ zu behaupten und muss nicht über seine eigene bekannte studentische Identität hinausgehen (Hyland 2002a: 1100).

2. Stating a purpose/goal: Diese Funktion umfasst im wesentlichen alle Formen der Textkommentierung und fällt damit weitestgehend mit Steinhoffs Verfasser-Ich und der writer role von Fløttum u. a. zusammen. Auch hier geht der Autor ein relativ geringes Risiko ein, er leitet einfach den Leser durch den Text. Daneben zählt Hyland zu dieser Funktion auch die Darstellung der in der Studie angestrebten Ziele und Absichten. Die Funktion stating a purpose/goal wird von den studentischen Autoren in Hylands Korpus verhältnismäßig häufiger verwendet als von erfahrenen Autoren (Hyland 2002a: 1101).

3. Explaining a procedure: Diese Funktion ähnelt der zweiten stark, auch was den Grad der persönlichen Bloßstellung betrifft, den die Studierenden ihr zuschreiben. Hier beschreibt der Autor seine Methoden und die Durchführung der Studie. Explaining a procedure ist die zweithäufigste Diskursfunktion, die in den studentischen Texten mit der ersten Person umgesetzt wird. Dabei scheinen die Studierenden nicht zu erkennen, dass sie mit dieser Funktion durchaus ihre wissenschaftliche Kompetenz betonen und ihre eigenen Entscheidungen bei der Umsetzung ihres wissenschaftlichen Projektes hervorheben (Hyland 2002a: 1102).

4. Elaborating an argument: Diese Funktion wird von Studierenden am seltensten in der ersten Person umgesetzt, was Hyland mit dem hohen Risikopotential erklärt, das sie enthält. Die Studierenden bevorzugten statt dessen unpersönliche Wendungen, um sich von ihren eigenen Argumenten und Überlegungen zu distanzieren, was bei den Lesern zu Frustrationen führe (Hyland 2002a: 1103). Fortgeschrittene Autoren jedoch setzen die erste Person hier verhältnismäßig häufig ein, um ihre Argumentation zu stärken.

5. Stating results/claims: Dies ist nach Hyland (Hyland 2002a: 1103) die riskanteste Funktion, in der die erste Person verwendet wird. Wissenschaftler benutzen sie, 
um eine bestimmte neue Aussage, eine Erweiterung des fachlichen Wissens, für sich zu beanspruchen und so ihren eigenen originären Beitrag explizit zu machen. Studierende dagegen bevorzugten es, sich von ihrem persönlichen Beitrag zurückzuziehen, um nicht Gefahr zu laufen, kritisiert oder widerlegt zu werden (Hyland 2002a: 1104).

Diese Kategorisierungen und ihre Beschreibungen deuten darauf hin, dass Hyland, ähnlich wie Tang und John (1999) annimmt, dass der Gebrauch der ersten Person zum Umsetzen dieser Diskursfunktionen ein kommunikatives Risiko für den Autor darstellt (s. z. B. Hyland 2002a: 1106). Dabei steigt das Risiko, je „verwundbarer“ sich der Autor macht, d. h. je stärker er sich der Kritik seiner Leser aussetzt (Hyland 2002a: 1104). Die riskanteste Handlung, die ein Autor in der ersten Person ausführen kann, ist nach Hyland, eigene Ergebnisse zu diskutieren und Behauptungen aufzustellen, die er vor der Community verteidigen muss (Stating results/claims). Die Vermeidung der ersten Person in dieser Funktion sei daher typisch für unerfahrene Autoren:

Clearly the writer invests most by using an authorial reference for this purpose [Stating results/claims, J. S.] and is also most vulnerable to criticism. Pledging your personal conviction in your results with a first person commitment is a risky strategy, and often one that novice writers lacked the confidence to take. (Hyland 2002a: S. 1104)

\subsubsection{Autorenrollen und wissenschaftliche Identität}

Die in diesem Kapitel beschriebenen Untersuchungen haben gezeigt, dass der Gebrauch der ersten Person ein elementarer Bestandteil wissenschaftlicher Texte ist und dass ein angemessener Einsatz der entsprechenden Pronomen beim Leser den Eindruck erzeugt, dass der Autor ein kompetenter wissenschaftlicher Autor und damit Wissenschaftler ist. Verschiedene Studien haben für den englischsprachigen Bereich sogar zwischen Low-risk und High-risk-Gebrauch (bezüglich der Machtausübung bzw. Face-Bedrohung) der ersten Person unterschieden und entsprechend die High-riskKontexte als Zeichen für eine stärkere, d.h. kompetentere wissenschaftliche Persona gewertet (z. B. Tang und John 1999; Hyland 2002a).

There is a continuum from not using ' $\mathrm{I}$ ' at all, through using ' $\mathrm{I}$ ' with verbs associated with the process of structuring the writing, to using ' $\mathrm{I}$ ' in association with the research process, and finally to using ' $\mathrm{I}$ ' with verbs associated with cognitive acts. Not using ' $\mathrm{I}$ ' at all gives the impression that the writer is withdrawing from all responsibility for the academic essay. None of my co-researchers do this. Using ' $\mathrm{I}$ ' in association with the process of structuring the essay still leaves room for its content being presented as if it were objective and factual. (Ivanič 1998: 307) 
In deutschsprachigen Texten scheint eine derartige Kategorisierung keine so deutliche Rolle zu spielen, zumindest wurde sie bisher noch nicht vorgeschlagen. Allerdings wird die erste Person in deutschen Texten auch insgesamt wesentlich seltener benutzt als in englischsprachigen Texten, auch wenn von einem generellen „Ich-Tabu“ keine Rede sein kann.

Dies deutet darauf hin, dass sich die Textkonventionen und vermutlich auch die diesen Konventionen zugrundeliegenden wissenschaftskulturellen Wertvorstellungen hinsichtlich des Gebrauchs der ersten Person zwischen dem deutschen und dem englischen stark unterscheiden. Wenn man bedenkt, dass gerade diese impliziten Wertvorstellungen beim Schreiben in der Fremdsprache häufig unreflektiert übertragen werden (vgl. z. B. Hufeisen|2002; Kaiser 2002; Eßer|1997), wird deutlich, dass hier Problemstellen liegen, die das Schreiben und v. a. das Publizieren in der Fremdsprache ggf. unerwartet erschweren können (Burrough-Boenisch 2003, 2005; Kaplan und Baldauf Jr. 2005).

Dabei ist auch zu beachten, dass man sowohl als Autor, als auch als kompetenter Leser wissenschaftlicher Texte, basierend auf der eigenen wissenschaftlichen Sozialisation offenbar sehr deutliche, allerdings häufig implizite, Vorstellungen davon hat, was einen Text „wissenschaftlich“ macht. Dies konnten beispielsweise Ken Hyland und Torsten Steinhoff nachweisen. Beide haben im Rahmen ihrer Untersuchung wissenschaftlicher und studentischer Texte auch andere Wissenschaftler dazu befragt, wie wissenschaftlich diese die Texte ihrer Korpora einschätzten (Steinhoff 2007; Hyland 2001b, 2002a). Steinhoffs Experten haben die studentischen Texte, die ihnen vorgelegt wurden, beispielsweise mit "ansatzweise wissenschaftlich“ (Steinhoff 2007, 160, 225, 383)), "kaum wissenschaftlich“ (Steinhoff 2007; 226) oder "größtenteils wissenschaftlich" (Steinhoff 2007, 298, 399) kommentiert. Hyland konnte zeigen, dass sich erfahrene Wissenschaftler beim Verfassen ihrer Texte sehr bewusst sind, dass sie sich in ihrem Text selber darstellen und entsprechend versuchen, den Eindruck, den ihre Leser von ihnen haben, zu steuern.

I'm very much aware that I'm building a facade of authority when I write, I really like to get behind my work and get it out there. Strong. Committed. That's the voice I'm trying to promote, even when I'm uncertain I want to be behind what I say. (Soc interview) (Hyland 2001b; 220)

All dies zeigt noch einmal, dass es notwendig ist, den Gebrauch der ersten Person in wissenschaftlichen Artikeln nicht nur aus rein (text)linguistischer, sondern auch aus interaktionaler Perspektive zu betrachten, um die wissenschaftkulturell unterschiedlichen Möglichkeiten der Autoren, sich eine überzeugende wissenschaftliche Identität zu konstruieren, zu untersuchen.

Aus den Ergebnissen der oben beschriebenen Studien lässt sich herauslesen, dass es im Wesentlichen zwei große Rollen sind, die in wissenschaftlichen Texten in der ers- 
ten Person realisiert werden, nämlich die des Schreibenden und die des im weiteren Sinne Forschenden bzw. wissenschaftlich Tätigen. Dies ist nicht weiter verwunderlich, da die primäre Identität, die im Rahmen eines wissenschaftlichen Artikels eine Rolle spielt und kommuniziert werden soll, die des "wissenschaftlichen Autors" ist, also eines Autors oder Textverfassers, der Wissenschaftler ist. Daneben gibt es offenbar noch vereinzelt vorkommende andere Rollen, die aber keinen so deutlichen gemeinsamen Nenner haben ${ }^{25}$, so dass alle Handlungen, die nicht einer dieser beiden Rollen zugeordnet werden können, im Folgenden in die Kategorie "Andere“ sortiert werden.

Die beiden wesentlichen Rollen werden hier in Anlehnung an Steinhoff als Verfasser und als Forscher bezeichnet (Steinhoff 2007). Diese sind äquivalent mit den oben beschriebenen Teilbereichen der Identität wissenschaftlicher Autor, nämlich Autor und Wissenschaftler. Hier besteht jedoch die Gefahr von Unklarheit, da das „Wissenschaftler Sein“ letztlich die übergeordnete Identitätsebene für diverse Teilidentitäten ist, weswegen im Folgenden auf Steinhoffs Terminologie zurückgegriffen wird (s. Abb. 3.3).

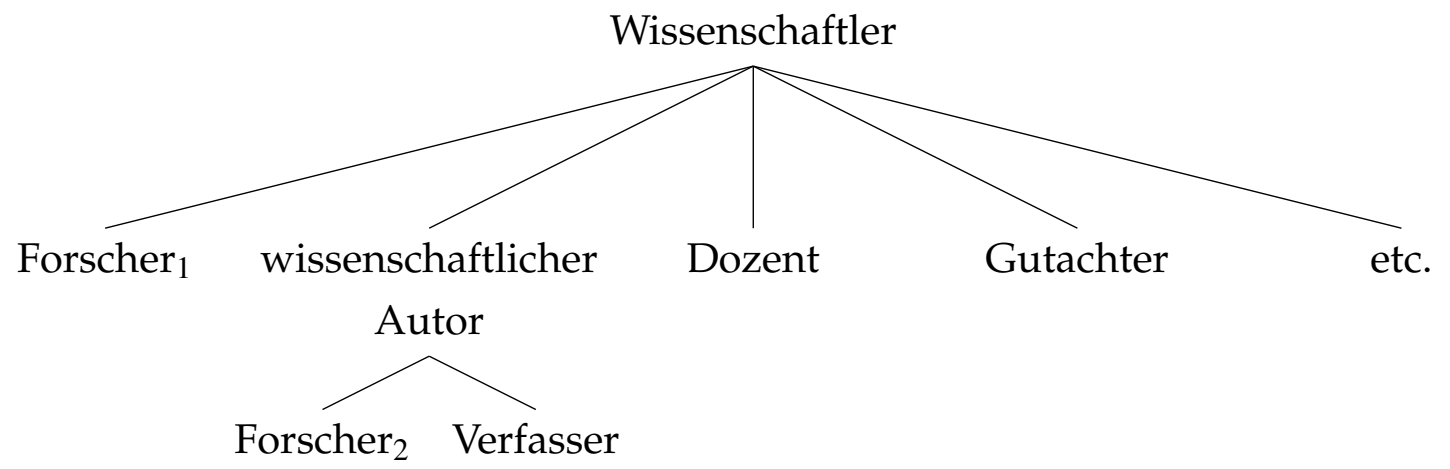

Abbildung 3.3: Teilidentitäten des „Wissenschaftler Seins“

Die parallele Benennung von Forscher ${ }_{1}$ und Forscher ${ }_{2}$ deutet hier darauf hin, dass die Forscherrolle, die ein Autor in seinem Text darstellt (Forscher 2 ), an der Forscherrolle, die er im (sog.) realen Leben ausfüllt (Forscher 1 ), orientiert ist. Im Text wird idealerweise der Forschungsprozess wiedergegeben, der auch tatsächlich stattgefunden hat (wenn auch häufig sicherlich geglättet und ggf. auch "geschönt“).

Dabei fallen unter die Rolle des Forschers vor allem diejenigen Handlungen, in denen der Forschungsprozess dargestellt wird, also vom Formulieren der Forschungsfrage über die Hypothesenbildung, die Datenerhebung, -aufbereitung und -analyse hin zum Darstellen der Ergebnisse. Daneben zählen aber auch und gerade die häufig als risikoreicher und face-bedrohender beschriebenen Handlungen in diesen Bereich, d.h. das Argumentieren für eigene Meinungen und Interpretationen sowie das Dar-

\footnotetext{
${ }^{25}$ Diejenigen anderen Rollen, die in den oben beschriebenen Untersuchungen identifiziert wurden, wurden auch vorrangig als studentische Gebrauchsweisen der ersten Person analysiert. Da das hier untersuchte Korpus ausschließlich aus veröffentlichten Texten besteht, ist daher anzunehmen, dass diese Rollen (Steinhoffs Erzähler-ICH, Hylands Expressing self-benefits und Tang/Johns I as the representative) hier nicht bzw. nicht so häufig auftreten.
} 
stellen neuer Informationen, also neuen Wissens, das von der Community aufgenommen werden soll. Diese Handlungen finden typischerweise alle außerhalb des Textes statt bzw. haben eine Wirkung, die über den Text hinausreicht.

Der Verfasser hingegen ist nicht so direkt mit dem Autor gleichzusetzen, da man als wissenschaftlicher Autor natürlich auch außerhalb seines Textes agiert, indem man beispielsweise mit Verlagen oder Korrektoren verhandelt usw. Im Gegensatz dazu zählen zu der Rolle des Verfassers diejenigen Handlungen, die innerhalb des Textes stattfinden und inhärent an das Medium „Text“ gekoppelt sind, d.h. die auf den Erstellungsprozess des Textes beschränkt sind bzw. die die Interaktion mit dem Leser umfassen. Dies sind vor allem metakommunikative/-diskursive Handlungen der Textstrukturierung und der Leserorientierung wie Vor- und Rückverweise, verbales und graphisches Darstellen etc.

Zur Übersicht sind die Ergebnisse aus den in den vorhergehenden Teilkapitel referierten Untersuchungen in den Tabellen 3.1 und 3.2 zusammengefasst und den entsprechenden Rollen, die im Weiteren benutzt werden, zugeordnet. Die Frage, die der folgenden Auswertung ganz allgemein zugrunde liegt, ist nun also, inwiefern sich die deutschen und US-amerikanischen Texte, die hier untersucht werden, bezüglich der sprachlich in ihnen dargestellten Rollen unterscheiden und ob sich ein weiter differenziertes Bild ergibt, wenn hier die Perspektive deutlich auf die Identitäts- und Rollenkonstruktion gelegt wird.

\subsection{Zusammenfassung}

In den vorhergehenden Kapiteln wurde der theoretische Hintergrund für die hier vorgestellte Untersuchung dargelegt. Grundlegend ist dabei die Annahme, dass wissenschaftliches Schreiben in einem soziokulturellen Kontext stattfindet und damit soziale Interaktion ist, auch wenn das Schreiben nicht als Face-to-face-Interaktion stattfindet.

Das wichtigste Ziel wissenschaftlicher Artikel ist es, neues Wissen zu vermitteln und idealerweise in der Community zu etablieren, indem der Autor seine Leser von seinen Behauptungen überzeugt. Dies kann jedoch nur erfolgreich geschehen, wenn der Leser den Autor grundsätzlich als vollwertiges Mitglied der Community akzeptiert. Daher ist es beim Verfassen wissenschaftlicher Texte notwendig, dass der Autor sich auf die richtige Art und Weise präsentiert. Besonders relevant wird die erfolgreiche Selbstdarstellung als kompetenter wissenschaftlicher Autor beispielsweise, wenn ein Text ein Peer Review-Verfahren durchlaufen muss, um veröffentlicht zu werden. Auch hier ist die gelungene Selbstdarstellung des Autors eine notwendige, aber natürlich keine hinreichende Bedingung dafür, dass der Text veröffentlicht wird, es geht hier selbstverständlich um Inhalte. Aber andersherum wird sicherlich kein Text veröffentlicht, in dem der Autor sich unangemessen, beispielsweise zu „studentisch“, präsen- 


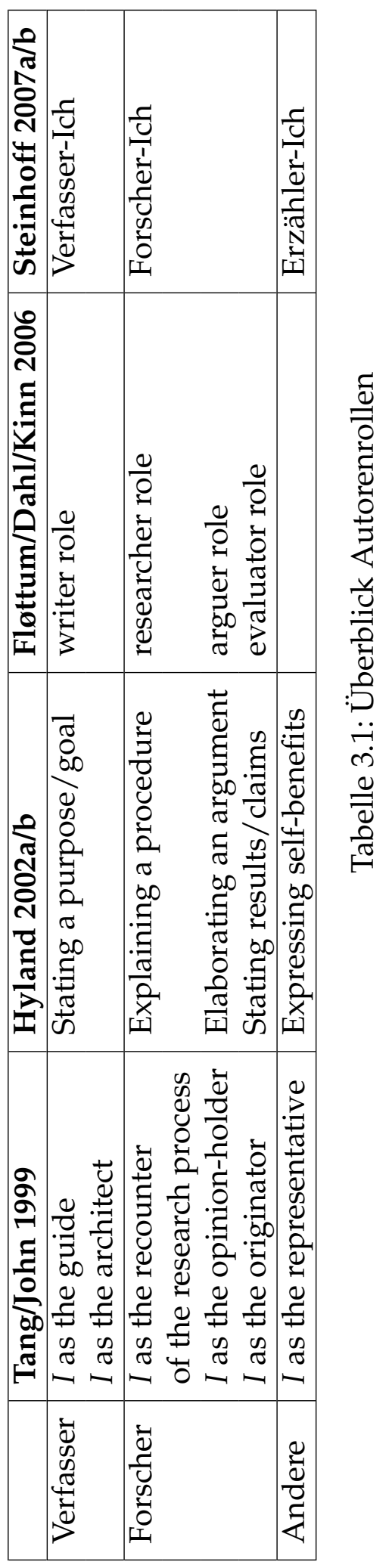




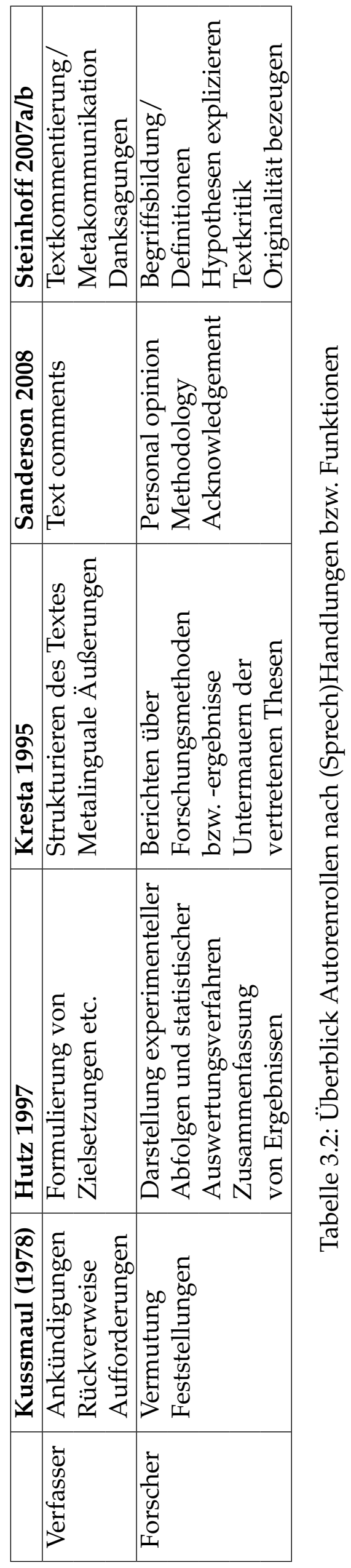


tiert. Hier wird der Text wohl einer umfassenden Überarbeitung unterzogen werden müssen, bevor er, wenn überhaupt, publiziert wird.

Der Erfolg eines Textes ist also nicht unwesentlich davon abhängig, ob der Leser die Art, wie sich der Autor in seinem Text darstellt, angemessen findet und die (angenommene) Person hinter dem Text als kompetenten und glaubwürdigen Wissenschaftler einstuft. Eine angemessene Selbstdarstellung ist die Grundlage, um den Leser von den Interpretationen und Ergebnissen, die im Text präsentiert werden, zu überzeugen 26 . Dass Leser sich ein derartiges Bild vom Autor machen und dass sie auch nachweisbare Kriterien haben, die sie den Autor als „,wissenschaftlich“ oder ",nicht-wissenschaftlich“ und damit "studentisch" einstufen lassen, zeigen beispielsweise die Experteneinschätzungen von Steinhoff (2007).

Die kompetente Selbstdarstellung, d.h. das überzeugende und glaubwürdige Auftreten des Autors in seinem Text, ist von wesentlicher Bedeutung für den Erfolg des Textes; sowohl, was die grundsätzliche Veröffentlichung betrifft, als auch seine überzeugende Wirkung. Diese Selbstdarstellung, d.h. die Konstruktion der Identität wissenschaftlicher Autor, findet auf verschiedenen sprachlichen Ebenen statt und Autoren haben diverse sprachliche Mittel zu Verfügung, von denen der Gebrauch der ersten Person Singular und Plural die offensichtlichsten sind.

Identitäten äußern sich über die Übernahme bzw. (Selbst-)Zuschreibung sozialer Rollen. Diese Rollen wiederum lassen sich anhand der Handlungen, die eine Person ausführt, nachzeichnen. Im geschriebenen Text, insbesondere in einem (üblicherweise) nicht-narrativen Genre wie dem wissenschaftlichen Artikel, sind dies die Handlungen, die der Autor sich selber zuschreibt, indem er sich als handelnde Person darstellt, so wie es in den Sätzen, die die erste Person Singular als Subjekt und Agens haben, der Fall ist.

Für wissenschaftliche Artikel sind insbesondere zwei soziale Rollen relevant, die die Identität „wissenschaftlicher Autor" ausmachen: der Forscher und der Verfasser. Darüber hinaus sind in unterschiedlichen Untersuchungen auch immer wieder vereinzelt Gebrauchsformen der ersten Person nachgewiesen worden, die auf andere Rollen schließen lassen, dies jedoch vor allem in studentischen Texten (Steinhoff 2007; Hyland 2002b). Innerhalb dieser Rollen können unterschiedliche typische Handlungen identifiziert werden, anhand derer die Rollen umgesetzt werden. Diese Handlungen, ebenso wie die generelle Häufigkeit, mit der die Pronomen der ersten Person benutzt werden, sind abhängig von der jeweiligen Wissenschaftskultur. Die Mitglieder einer bestimmten Wissenschaftskultur haben im Rahmen ihrer wissenschaftlichen Sozialisation Maßstäbe entwickelt, anhand derer sie ihre eigenen Texte verfassen und die Texte anderer Autoren bewerten. Beim Übergang zwischen zwei Kulturen kann es jedoch dazu kommen, dass die Maßstäbe widersprüchlich sind und erlernte Konventionen,

\footnotetext{
${ }^{26}$ Natürlich können Leser von den Schlussfolgerungen auch nicht überzeugt sein, obwohl sie den Autor als kompetenten Wissenschaftler anerkennen.
} 
z. B. zum Gebrauch der ersten Person und damit zur Konstruktion einer angemessenen Autorenidentität, die in die L2 übertragen werden, nicht die gewünschte Konnotation haben.

Mehrere Untersuchungen haben gezeigt, dass es, bezogen auf den Gebrauch der Pronomen der ersten Person, deutliche Unterschiede zwischen dem Englischen und dem Deutschen gibt. In der folgenden Analyse eines Korpus aus deutschen und USamerikanischen Texten werden diese Unterschiede aus interaktionaler Perspektive und in Hinblick auf die Mechanismen der Identitätskonstruktion überprüft. Die grundlegende Frage ist dabei, inwiefern sich der Gebrauch der ersten Person in den hier untersuchten deutschen und englischen Texten unterscheidet, d.h. mit welchen Handlungen die Verfasser- und Forscherrolle jeweils dargestellt werden. Dazu werden sowohl die erste Person im Singular als auch im Plural berücksichtigt. Darüber hinaus wird der Gebrauch des unpersönlichen MAN/ONE als wichtigste syntaktisch vergleichbare Alternative betrachtet. Es ist zu erwarten, dass das ICH in den englischen Texten nicht nur öfter, sondern auch in anderen Funktionen benutzt wird, als in den deutschen. Statt dessen werden in den deutschen Texten voraussichtlich das WIR und das MAN einige der Handlungen übernehmen, die im Englischen das ICH erfüllt.

Im anschließenden Kapitel werden zunächst das Korpus und die angewandte Analysemethode vorgestellt. In der darauf folgenden Auswertung werden dann die drei erwarteten Rollenkategorien mit den entsprechenden Unterkategorien bzw. den die Rollen konstituierenden Handlungen an Beispielen vorgestellt und danach die Ergebnisse aus den beiden Korpora verglichen. Im Anschluss daran wird ein sehr untypischer Text als Fallstudie analysiert und als Abschluss des empirischen Teils wird der ICH-Gebrauch zudem im Rahmen der sozialpsychologischen Theorie des Impression Managements beleuchtet. Anschließend werden mögliche didaktische Auswirkungen der Ergebnisse diskutiert. 


\section{Kapitel 4}

\section{Das Korpus}

Diese Arbeit soll Unterschiede in deutschen und englischsprachigen wissenschaftlichen Artikeln des Faches Soziologie in Bezug auf die Selbstdarstellung und die Konstruktion einer angemessenen wissenschaftlichen Identität des Autors anhand verschiedener sprachlicher Mittel aufzeigen. Dazu wurde ein Korpus aus soziologischen Fachartikeln erstellt, das Rückschlüsse auf diese Fragestellung zulässt.

Das Fach Soziologie wurde aus verschiedenen Gründen gewählt. Zum einen ist es ein typischer Vertreter der Sozialwissenschaften, d. h. die Arbeitsweise in diesem Fach orientiert sich an sozialwissenschaftlichen Methoden, es gibt im empirischen Bereich qualitative und quantitative Ausprägungen und auch die Theoriebildung wird intensiv verfolgt. Diese unterschiedlichen Ausrichtungen spiegeln sich auch in den hier zur Untersuchung ausgewählten Texten wider.

Zum anderen wurden die Sozialwissenschaften zwar in der englischsprachigen Forschung zum Gebrauch der ersten Person in wissenschaftlichen Artikeln berücksichtigt (Hyland 2000, 2002a, 2011; Fløttum u. a. 2006), im deutschsprachigen Kontext hat sich die Forschung bisher jedoch stärker auf die Geisteswissenschaften konzentriert (Kussmaul 1978; Kresta 1995; Hutz 1997; Sanderson 2008a). Und nicht zuletzt wurde das Fach auch ausgewählt, da in den Sozialwissenschaften, anders als z. B. in naturwissenschaftlichen Arbeiten, Sprache und die sprachliche Darstellung eine bedeutende Rolle spielen. Artikel, die in der Soziologie geschrieben werden, sind verbos und die sprachliche Gestaltung steht auch in quantitativen Untersuchungen im Vergleich z. B. zu Formeln, graphischen oder tabellarischen Darstellungen eindeutig im Vordergrund. Demgegenüber ist die Soziologie aber keine Wissenschaft, die genuin Sprache als ihren eigentlichen Gegenstand hat, so wie die Linguistik oder die Philologien. Aus diesem Grund kann man davon ausgehen, dass die Sprache der Darstellung hier deutlicher als Werkzeug zu werten ist als in literaturwissenschaftlichen oder linguistischen Artikeln.

Anders als in vielen anderen Untersuchungen, die in diesem Themenbereich bisher durchgeführt wurden, wird hier nur eine Disziplin in zwei verschiedenen (wis- 
senschafts)kulturellen Ausprägungen untersucht. Daher stehen hier eindeutig (inter)kulturelle Unterschiede in den Möglichkeiten der Selbstdarstellung im Vordergrund. Dabei gehe ich davon aus, dass sich disziplinäre Unterschiede auch zwischen den einzelnen Fächer des Fachbereichs „Sozialwissenschaften“ bemerkbar machen und dass es sinnvoll ist, ein einzelnes Fach zu fokussieren, wenn es um die Identifikation von Unterschieden gehen soll, die auf national- bzw. sprachkulturellen Unterschieden beruhen. Es bleibt jedoch zu berücksichtigen, dass das Fach Soziologie in sich keineswegs als homogen zu betrachten ist. Es gibt ein weites Kontinuum an Fragestellungen und Ausrichtungen, die sich zwischen einem weitgehend statistisch arbeitenden quantitativen und einem interpretativ-hermeneutisch arbeitenden qualitativen Pol bewegen. Dieses Kontinuum ist auch in den ausgewählten Korpustexten repräsentiert (s. Tabelle 4.5 auf S. 96.

Das für diese Arbeit verwendete Korpus besteht aus insgesamt 50 Texten und umfasst 565851 Wörter (s. Tab. 4.2 und 4.3 auf S. 95 bzw. 96). Es setzt sich zusammen aus zwei Einzelkorpora mit jeweils 25 Texten aus einer deutschen und einer US-amerikanischen soziologischen Fachzeitschrift. Basierend auf den Auswertungen des Social Science Citation Index ${ }_{1}^{1}$ wurde jeweils die führende Zeitschrift der beiden Länder ausgewählt, für Deutschland die Kölner Zeitschrift für Soziologie und Sozialpsychologie und für die USA das American Journal of Sociology.

Die „Kölner Zeitschrift für Soziologie und Sozialpsychologie“ (KZfSS) ist eine Fachzeitschrift mit aktuellen Beiträgen über die sozialwissenschaftliche und sozialpsychologische Forschung. Sie wurde 1948 von dem Kölner Soziologen Leopold von Wiese als „Kölner Zeitschrift für Soziologie“ gegründet und umfasst seit 1954 auch sozialpsychologische Themenstellungen $u$.a. aus der Kultursoziologie und der qualitativen Sozialforschung. Die Hefte erscheinen im VS Verlag vierteljährlich im März, Juni, September und Dezember, daneben erscheinen jährlich thematische Sonderhefte, die jedoch in der Zusammenstellung des Korpus nicht berücksichtigt wurden. Die KZfSS gilt nach Verbreitung und Größe als das bedeutendste soziologische Fachorgan im deutschsprachigen Raum und berichtet umfassend über alle Fachrichtungen der deutschsprachigen soziologischen Forschung sowie über die soziologische Forschung weltweit. Dabei ist sie grundsätzlich als soziologische Universal-Zeitschrift ausgelegt, hat aber auch einen ausgeprägten empirischen Fokus, der sowohl qualitative als auch quantitative Arbeiten umfasst $t^{2}$,

In der KZfSS werden jährlich über 40 Aufsätze ausschließlich als Erstveröffentlichungen überwiegend auf Deutsch, jedoch immer mit englischem Abstract, publiziert. Daneben umfasst diese Zeitschrift ausführliche Literaturberichte sowie einen umfang-

\footnotetext{
${ }^{1}$ s. http://thomsonreuters.com/products_services/science/science_products/a-z/social_sciences_ citation_index/. Zugriff: 29.08.2011

${ }^{2}$ s. http://en.wikipedia.org/wiki/K\%C3\%B6lner_Zeitschrift_f\%C3\%BCr_Soziologie_und_ Sozialpsychologie, Zugriff: 24.01 .2011
} 
reichen Rezensionsteil mit Besprechungen der deutschen und internationalen Fachliteratur. Nach Angaben der Homepage der Zeitschrift im VS-Verlag ${ }^{3}$ wird besonderer Wert darauf gelegt, auch dem wissenschaftlichen Nachwuchs eine Möglichkeit für die Erstveröffentlichung zu bieten. Im Jahr 2008/2009 waren 83 Reviewer an der Begutachtung von Manuskripten beteiligt, darunter auch einige der in dem hier bearbeiteten Korpus vertretenen Autor/innen 4 Jährlich werden ca. 25 bis 40 Prozent der eingereichten Manuskripte angenommen und veröffentlicht, die Mehrzahl der Manuskripte muss nach eigenen Angaben auf der Homepage der Zeitschrift $t^{5}$ von den Autoren nochmals überarbeitet werden.

Das American Journal of Sociology (AJS) wurde 1895 in Chicago gegründet und vertritt das Fach ebenfalls in seiner Gesamtheit, mit einem Schwerpunkt auf Theoriebildung und innovativen Methoden. . $^{6}$ ist auch heute noch dem renommierten soziologischen Institut der Universität Chicago zugeordnet und gilt als eine der weltweit meistgelesenen Zeitschriften des Faches Soziologie. Die Zeitschrift richtet sich vor allem an Soziologen, umfasst aber auch soziologisch ausgerichtete Beiträge aus den angrenzenden Wissenschaften wie Ethnologie, Statistik, Politikwissenschaften oder Geschichtswissenschaften 7 Neben Aufsätzen enthalten die zweimonatlich erscheinenden Ausgaben des AJS auch eine umfassende Rezensionssektion, in der die wichtigsten Neuerscheinungen des Faches besprochen werden. Qualifizierte Artikel werden nach einem doppelt verdeckten Begutachtungsverfahren ausgewählt und nach eigenen Angaben publiziert das AJS nur einen „sehr kleinen Prozentsatz“ der eingereichten Arbeiten 8

Um einen Überblick über die aktuelle Situation zu gewinnen, wurden als Gesamtmenge der relevanten Artikel alle veröffentlichen Artikel der Jahre 2005 bis (März) 2010 festgelegt, die von einem Einzelautor verfasst wurden, der Muttersprachler der entsprechenden Sprache ist $9^{9}$ und den Hauptteil seiner akademischen und in den meisten Fällen auch schulischen Ausbildung im jeweiligen Land absolviert hat. Dies wurde durch Internetrecherche verifiziert. Darüber hinaus wurden in die Grundgesamtheit nur Texte aufgenommen, deren Autoren entweder an Universitäten oder anderen Forschungseinrichtungen eindeutig im Bereich Soziologie verortet sind bzw. es zum Zeitpunkt der Veröffentlichung ihres Artikels waren. Die Autoren sind also alle sowohl in dem Land als auch in der Sprache wissenschaftlich sozialisiert, in der sie die Artikel

${ }^{3}$ s. http://www.vsjournals.de/index.php;do=viewmag/sid=15379164524d3ef33681a48605821277/ site $=\mathrm{kzfss} / \mathrm{lng}=\mathrm{de} / \mathrm{area}=\mathrm{soz} / \mathrm{id}=4 /$ alloc $=/$ full $=1$, Zugriff: 24.01 .2011

${ }^{4}$ s. http://www.uni-koeln.de/kzfss/ks-gutachter.htm, Zugriff: 24.01 .2011

${ }^{5}$ s. http://www.uni-koeln.de/kzfss/konventionen/ksents.htm. Zugriff: 24.01.2011

${ }^{6}$ s. http://www.jstor.org/page/journal/amerjsoci/about.html, Zugriff: 24.01.2011

${ }^{7}$ s. http://www.jstor.org/page/journal/amerjsoci/about.html, Zugriff: 24.01.2011

${ }^{8}$ s. http://www.jstor.org/page/journal/amerjsoci/about.html, Zugriff: 24.01.2011

${ }^{9} \mathrm{Zu}$ Problemen bezüglich der eindeutigen Bestimmung, ob ein Autor tatsächlich Muttersprachler der jeweiligen Sprache ist, s. Fløttum u. a. (2006 1-2), zu Problemen des Begriffs Muttersprache an sich s. z. B. Ahrenholz (2008) 
geschrieben und veröffentlicht haben. Außerdem sind sie fest in der Disziplin verortet und sind als Mitglieder der Community anerkannt und ausgewiesen. Aus dieser Grundgesamtheit, die 44 deutsche und 59 amerikanische Texte umfasst, wurden die im Korpus verwendeten Artikel zufällig ausgewählt.

\begin{tabular}{|l|l|l|}
\hline & Einzelautoren gesamt & L1 \\
\hline KZfSS & 56 & 44 \\
\hline AJS & 83 & 59 \\
\hline
\end{tabular}

Tabelle 4.1: Anzahl Texte

In Bezug auf die Größe des Korpus wurden andere Forschungen, die ähnliche Fragestellungen bearbeitet haben, als Maßstab zugrunde gelegt. Grundsätzlich ist das Korpus mit 50 Texten, d.h. 25 Texten pro Teilkorpus, und ca. einer halben Millionen Wörtern verhältnismäßig klein. In der KIAP-Studie (Fløttum u. a. 2006) wurden beispielsweise insgesamt 450 Texte untersucht, Ken Hyland (z. B. Hyland 2001b, 2002a) hat 240 Forschungsartikel und 64 studentische Abschlussarbeiten bearbeitet und das SCEGAD-Korpus von Sanderson enthält 100 Texte (Sanderson 2008a: 65). Dabei ist jedoch zu bedenken, dass diese Projekte inhaltlich (und meist auch personell) wesentlich breiter angelegt waren. Diese Korpora enthalten Texte aus verschiedenen Disziplinen und teilweise unterschiedlichen Sprachen sowie Texte, die von mehreren Autoren verfasst wurden. Wenn man diese größeren Korpora anhand der hier relevanten Kriterien (Sprache, Disziplin, Einzelautor, wissenschaftliche Veröffentlichung im Gegensatz zu studentischen Texten) aufschlüsselt, ergeben sich vergleichbare Größenordnungen.

Abgesehen davon richtet sich die Größe immer auch nach pragmatischen Gesichtspunkten und ist abhängig von der Forschungsfrage und den angewandten Methoden (McEnery u.a. 2006). Beispielsweise konnte der erste Schritt dieser Untersuchung, die Lokalisierung von Sätzen mit einem spezifischen Subjekt(pronomen) automatisch durchgeführt werden, aber die so erhaltenen Treffer mussten danach im Einzelnen manuell überprüft werden. Viele der Sätze mit den Pronomen der ersten Person Singular und Plural im Subjekt mussten aussortiert werden, da sich in diesen Fällen die Pronomen nicht auf den Autor bezogen, sondern z. B. einen Interviewausschnitt wörtlich wiedergaben. Dies hat die mögliche Größe eines bearbeitbaren Korpus maßgeblich bestimmt.

In direktem Zusammenhang mit der Größe eines Korpus steht oft auch die Frage nach der Repräsentativität einer Untersuchung. Wann und unter welchen Umständen ist ein Korpus repräsentativ, und repräsentativ wofür? Und was genau heißt eigentlich „Repräsentativität“ in Bezug auf linguistische Untersuchungen anhand von (wie auch immer gearteten) Korpora? McEnery u. a. (2006) definieren das Konzept in Anlehnung an Leech (1991: 27) folgendermaßen: 
[A] corpus is thought to be representative of the language variety it is supposed to represent if the findings based on its contents can be generalized to the said language variety. (McEnery u. a. 2006) 13)

Grundsätzlich ist die hier vorliegende Studie exemplarisch gedacht und erhebt keinerlei Anspruch auf eine umfassende Repräsentativität. Nichtsdestoweniger ist davon auszugehen, dass die Aussagen, die hier getroffen werden, sicherlich eine unterschiedliche Relevanz und Repräsentativität in Bezug auf unterschiedliche Populationen haben. Beispielsweise kann man anhand des hier untersuchten Samples sicherlich nicht auf "das wissenschaftliche Schreiben" an sich oder auch nur "das Schreiben in den Sozialwissenschaften" generalisieren. Dazu ist das Korpus weder ausreichend groß, noch umfassend genug, beispielsweise enthält es keine ausreichende Variation der in den Sozialwissenschaften üblichen Textsorten. Weiterhin müssten dafür auch andere Disziplinen aus diesem Fachbereich untersucht werden und sicherlich wäre es ebenfalls notwendig, Texte mit mehr als einem Autor einzuschließen, wie es in anderen Untersuchungen gehandhabt wurde (vgl. z. B. Fløttum u. a. 2006; Sanderson 2008a). Da es hier jedoch um die Konstruktion von Rollen und Identitäten geht und diese Rollen im wissenschaftlichen Schreiben auch von der Textsorte abhängig sein können (der Autor eines Einführungswerkes hat z. B. eine andere Rolle als der Autor einer Rezension), beschränkt sich diese Untersuchung exemplarisch auf eine einzelne Textsorte.

Gute Aussagen können dagegen wohl bezüglich der von Einzelautoren im untersuchten Zeitraum in den beiden untersuchten Zeitschriften veröffentlichten Forschungsartikel getroffen werden. Dies mag auf den ersten Blick vielleicht nicht vorrangig relevant erscheinen, aber da es sich bei den beiden Zeitschriften um anerkannte und hoch gerankte Organe handelt, die im Fach Soziologie ein hohes Ansehen und damit auch einen ernstzunehmenden Einfluss auf die Meinungsbildung des Faches haben (sowohl inhaltlich als auch stilistisch), ist davon auszugehen, dass die hier erzielten Ergebnisse über diesen Umweg auch Aussagen über das wissenschaftliche Schreiben im Fach Soziologie an sich zulassen.

Diese Zeitschriften tragen großes Prestige und es gilt als wissenschaftlicher Erfolg, einen oder mehrere Artikel in den führenden Zeitschriften des Faches veröffentlicht zu haben. Eine Veröffentlichung stellt also erhebliches symbolisches bzw. soziales Kapital dar (vgl. z. B. Weingart 2003: 48-49). Entsprechend lässt sich annehmen, dass Artikel, die in diesen Zeitschriften veröffentlicht wurden, sowohl inhaltlich als auch stilistisch den typischen Anforderungen an wissenschaftliche Artikel im Fach Soziologie entsprechen bzw. diese Anforderungen zumindest teilweise sogar definieren. Was von den Herausgebern und Reviewern dieser Zeitschriften als veröffentlichungswürdig akzeptiert wurde, entspricht den Regeln der jeweiligen Zeitschrift und wirkt aufgrund der führenden Position dieser Organe innerhalb des Faches damit auch wieder als Vorbild, nach dem sich andere Autoren und andere Zeitschriften richten können. Dadurch ver- 
fügen die Herausgeber und Reviewer dieser Zeitschriften über eine große kulturelle Macht. Aus diesem Grund wurden diese Zeitschriften als Beispiele dafür ausgewählt, welche Stile in den beiden soziologischen Communities Deutschlands und der USA anerkannt sind.

In diesem Zusammenhang ist es auch notwendig, darauf hinzuweisen, dass publizierte Artikel nicht ohne Weiteres als das Produkt einer einzigen Person betrachtet werden können, auch wenn letztlich nur ein verantwortlicher Autor angegeben wird (s. z. B. Jakobs 1997). Die hier untersuchten Texte haben ein Peer Review-Verfahren durchlaufen, in dessen Verlauf die Autoren sehr wahrscheinlich Änderungsvorschläge und andere Hinweise zu ihren Texten erhalten haben, und es ist nicht nachvollziehbar, ob, wie und wo sich diese Hinweise, ebenso wie die Guidelines für Autoren und Feedback aus anderen Quellen, auf die hier untersuchten Mittel und Zusammenhänge ausgewirkt haben. Da es hier jedoch darum gehen soll, die fertigen, d.h. die in den jeweiligen wissenschaftskulturellen Zusammenhängen publizierten Produkte zu vergleichen, wirkt sich dieser Einfluss nicht direkt auf die Interpretation der Ergebnisse aus. Dennoch wird noch einmal deutlich, dass es hier in erster Linie nur darum gehen kann, die beiden ausgewählten Samples zu vergleichen und alle weiterführenden Schlussfolgerungen mit Vorsicht vorzunehmen, ein Problem, dass auch andere Untersuchungen, die mit publizierten Texten gearbeitet haben, betrifft:

Another source of interference which should be pointed out is the guidelines for authors and editorial policies of the various journals. We do not regard the language of the texts in our corpus simply as the authors' own preferred language use. Rather, we see it as the result of complex interactions between the authors, written guidelines, referees and journal editors. [...] It is important to realise that the claims we make about the linguistic usage of the language-discipline populations (that we study by means of our sample corpus) are claims about the language that authors use in the context of publishing in research journals - and that context includes the demands and censorship of referees and editors. This also, unfortunately, adds to the complexity of the question about representativity, for it is possible that our samples are skewed in different directions with respect to the degree and nature of such editorial interference. (Fløttum u.a.2006: 11)

Weiterhin werden die beiden Sprachen Deutsch und Englisch durch Artikel aus nur jeweils einer deutschen und einer US-amerikanischen Fachzeitschrift repräsentiert, was letztlich ebenfalls die Übertragbarkeit der Ergebnisse einschränkt. Es stellt sich die Frage, inwieweit Rückschlüsse auf das Schreiben in Großbritannien, Australien, Österreich, der (deutschsprachigen) Schweiz und anderen Ländern, in denen eine Varietät dieser Sprachen gesprochen wird, gezogen werden können. 
Grundsätzlich gehe ich hier davon aus, dass sich die Ergebnisse nicht ohne Weiteres übertragen lassen, da sich die Wissenschaftskulturen in diesen Ländern basierend auf z. B. bildungs- und wissenschaftspolitischen Einstellungen, dem Schul- und Universitätssystem, stärkeren Einflüssen aus anderssprachigen Wissenschaftstraditionen etc. von der deutschen bzw. der US-amerikanischen Wissenschaftskultur unterscheiden und dass sich diese Unterschiede auch in den jeweiligen Schreibstilen abzeichnen ${ }^{10}$ Um zu diesen Varietäten brauchbare Aussagen treffen zu können, müssten Texte aus diesen Wissenschaftskulturen untersucht werden, was in dem hier bestehenden Kontext nicht zu leisten ist 11 Daher werden hier nur Texte aus einer deutschen und einer US-amerikanischen Zeitschrift betrachtet, auch wenn aus Gründen der besseren Lesbarkeit hier von "deutschen“ und „englischen“ Texten die Rede ist.

Die für diese Arbeit verwendeten Texte sind in den Tabellen 4.2 und 4.3 zusammengefasst, ausführliche Literaturangaben stehen im Anhang. Die beiden Einzelkorpora haben eine Wortzahl von 377604 für das englische bzw. 188247 für das deutsche Teilkorpus, was eine Gesamtwortzahl von 565851 Wörtern ergibt. Die Texte wurden von 17 (englisch) bzw. 15 (deutsch) männlichen und 8 bzw. 10 weiblichen Autoren und Autorinnen verfasst (s. Tabelle 4.4).

In beiden Korpusteilen sind sowohl Texte vorhanden, die auf einer qualitativ ausgerichteten Forschung basieren, als auch solche, die über eine quantitative Forschung berichten. Darüber hinaus sind einige Texte auch rein theoretisch ausgerichtet, $d . h$. sie basieren nicht auf einer konkreten empirischen Untersuchung. Im englischen Teilkorpus werden daneben auch empirische Studien referiert, in denen sowohl quantitative als auch qualitative Methoden angewandt wurden, diese werden hier als "gemischt" bezeichnet. Die Verteilung der Korpustexte nach diesen Parametern wird in Tabelle 4.5 wiedergegeben.

Ein weiterer Parameter, der in der Analyse betrachtet werden soll, ist die Schreiberfahrung bzw. der Grad der Sozialisation in die Wissenschaftskultur der Autoren, was anhand des akademischen Status operationalisiert wird. Die direktere Operationalisierung anhand der veröffentlichten Arbeiten hat sich als nicht umsetzbar herausgestellt, da insbesondere bei Autoren mit vielen Veröffentlichungen auf Instituts- oder auch persönlichen Websites häufig nur eine Auswahl ihrer Arbeiten angegeben wird und auch über diverse bibliographische Kataloge nur unvollständige Informationen verfügbar sind.

\footnotetext{
${ }^{10}$ Dabei ist natürlich zu berücksichtigen, dass auch diese Einteilung im Grunde willkürlich ist, wenn man davon ausgeht, dass der Begriff "Kultur" ein "Zoombegriff" ist (s. Abschnitt 2.2.3). So wäre es sicherlich auch vorstellbar, dass es bestimmte "Institutskulturen“ gibt (z. B. basierend auf den Vorstellungen einzelner Dozenten oder Professoren), die sich ggf. auch im Schreiben ihrer Studierenden und Alumni widerspiegeln.

${ }^{11}$ Allerdings ist es gut möglich, dass sich in einer entsprechenden Untersuchung keine gravierenden Unterschiede zeigen. Sanderson (2008a 116) konnte beispielsweise für die Verwendung der Pronomen der ersten Person als grammatisches Subjekt keine signifikanten Unterschiede zwischen britischem und US-amerikanischem Englisch nachweisen.
} 
Als akademische Statuspositionen im deutschen Kontext haben sich aus dem Korpus recht eindeutig die folgenden ergeben: Graduierte ohne Promotion (M.A., Dipl. Soz.), Promovierte (Dr. Phil., PhD, meist als wissenschaftliche Mitarbeiter oder Räte), Privatdozenten bzw. Habilitierte ohne Professur (PD Dr. Phil., PD Dr. rer. pol.) und Professoren (Prof. Dr. phil., Prof. Dr. rer soc., Prof. Dr. rer. pol). Die US-amerikanischen Entsprechungen, die in den biografischen Angaben zu den Korpustexten genannt wurden, sind Doctoral Student, Assistant Professor, Associate Professor und Full Professor (s. Tabelle 4.6.).

\begin{tabular}{|l|l|l|}
\hline Name & Jahr & Anzahl Wörter \\
\hline Bartley & 2007 & 16840 \\
\hline Chen & 2006 & 19936 \\
\hline Gerber & 2006 & 15510 \\
\hline Gibson & 2005 & 12646 \\
\hline Grodsky & 2007 & 14507 \\
\hline Kay & 2005 & 15272 \\
\hline King & 2008 & 13461 \\
\hline Lachmann & 2009 & 11405 \\
\hline Loveman & 2005 & 24185 \\
\hline Lyons & 2007 & 11495 \\
\hline Madsen & 2009 & 17356 \\
\hline Martin & 2008 & 9847 \\
\hline Menjivar & 2006 & 15214 \\
\hline Pedriana & 2006 & 15776 \\
\hline Quinn & 2008 & 16437 \\
\hline Sampson & 2008 & 13570 \\
\hline Schrank & 2008 & 10199 \\
\hline Schwartz & 2010 & 10313 \\
\hline Skaggs & 2008 & 11485 \\
\hline Steensland & 2006 & 20856 \\
\hline Straughn & 2005 & 21520 \\
\hline Vaisey & 2009 & 13244 \\
\hline Vallas & 2006 & 15140 \\
\hline Vaughan & 2006 & 17444 \\
\hline Viterna & 2006 & 13946 \\
\hline & gesamt & 377604 \\
\hline & & \\
\hline
\end{tabular}

Tabelle 4.2: Texte aus dem American Journal of Sociology 


\begin{tabular}{|l|l|l|}
\hline Name & Jahr & Anzahl Wörter \\
\hline Albert & 2005 & 3405 \\
\hline Becker & 2005 & 8445 \\
\hline Berninger & 2009 & 8090 \\
\hline Best & 2008 & 7512 \\
\hline Bienfait & 2006 & 7861 \\
\hline Brose & 2008 & 5635 \\
\hline Gerhards & 2008 & 8090 \\
\hline Helbig & 2010 & 5422 \\
\hline Isengard & 2005 & 6904 \\
\hline Kern & 2007 & 10593 \\
\hline Lohmann & 2010 & 8421 \\
\hline Lois & 2008 & 7709 \\
\hline Nollmann & 2006 & 6836 \\
\hline Nonnenmacher & 2007 & 6687 \\
\hline Otte & 2005 & 9863 \\
\hline Pollmann-Schuldt & 2006 & 4957 \\
\hline Rössel & 2006 & 5111 \\
\hline Schnabel & 2005 & 11469 \\
\hline Schulz-Schaeffer & 2009 & 9462 \\
\hline Schwinn & 2006 & 11344 \\
\hline Stauder & 2008 & 6382 \\
\hline Strauß & 2009 & 5551 \\
\hline Strein & 2005 & 8596 \\
\hline Teipen & 2008 & 6174 \\
\hline Trappe & 2006 & 7728 \\
\hline & $\mathbf{g e s a m t}$ & $\mathbf{1 8 8 2 4 7}$ \\
\hline & & \\
\hline
\end{tabular}

Tabelle 4.3: Texte aus der Kölner Zeitschrift für Soziologie und Sozialpsychologie

\begin{tabular}{|l|l|l|}
\hline & männlich & weiblich \\
\hline KZfSS & 15 & 10 \\
\hline AJS & 17 & 8 \\
\hline
\end{tabular}

Tabelle 4.4: Verteilung nach Geschlecht

\begin{tabular}{|l|l|l|l|l|}
\hline & qualitativ & quantitativ & theoretisch & gemischt \\
\hline KZfSS & 2 & 16 & 7 & 0 \\
\hline AJS & 14 & 8 & 1 & 3 \\
\hline
\end{tabular}

Tabelle 4.5: Verteilung nach Forschungsperspektive

\begin{tabular}{|l|l|l|l|l|}
\hline & $\begin{array}{l}\text { nicht promo- } \\
\text { viert }\end{array}$ & $\begin{array}{l}\text { promoviert } \\
\text { bzw. Assistant } \\
\text { Prof. }\end{array}$ & $\begin{array}{l}\text { habilitiert } \\
\text { bzw. Associa- } \\
\text { te Prof. }\end{array}$ & (Full) Prof. \\
\hline KZfSS & 6 & 10 & 4 & 5 \\
\hline AJS & 1 & 15 & 3 & 6 \\
\hline
\end{tabular}

Tabelle 4.6: Verteilung nach akademischem Status als Hinweis auf die Schreiberfahrung 


\begin{tabular}{|l|l|l|}
\hline & Englisch & Deutsch \\
\hline männlich & 253308 & 113552 \\
\hline weiblich & 124296 & 74695 \\
\hline nicht promoviert & 16437 & 40447 \\
\hline $\begin{array}{l}\text { promoviert/Assistant } \\
\text { Prof. }\end{array}$ & 154352 & 71462 \\
\hline $\begin{array}{l}\text { Privatdozent/Associate } \\
\text { Prof. }\end{array}$ & 41189 & 33735 \\
\hline Full) Professor & 90425 & 42603 \\
\hline qualitativ & 106663 & 16767 \\
\hline quantitativ & 193006 & 113112 \\
\hline andere & 77935 & 58368 \\
\hline
\end{tabular}

Tabelle 4.7: Verteilung Wörter nach zusätzlichen Parametern 


\section{Kapitel 5}

\section{Auswertung}

Identität im wissenschaftlichen Schreiben zeigt sich am deutlichsten am Gebrauch von Personalpronomen der ersten Person (vgl. z. B. Fløttum u. a. 2006; Sanderson 2008a), weshalb diese auch im Fokus der vorliegenden Arbeit stehen. Im qualitativen Teil wird untersucht, welche sozialen Rollen die Autoren in der ersten Person Singular und Plural sowie mit dem sog. unpersönlichen MAN ausdrücken. Die Zuschreibung bestimmter Handlungen anhand der Verben und Verbkomplexe, mit denen die erste Person kombiniert wird, gibt Aufschluss darüber, in welchen sozialen Rollen sich ein Wissenschaftler in seinem Artikel darstellt, um die relevanten Eigenschaften wie „überzeugend", "glaubwürdig“, ,"kompetent" usw. zu transportieren. Es ist davon auszugehen, dass aus allen möglichen Rollen nur ein kleiner Teil benutzt wird, da andere in diesem Kontext wahrscheinlich weniger überzeugend wirken. Dabei muss jeder Autor immer wieder neu die Entscheidung treffen, welche Art der Selbstdarstellung und welche seiner Eigenschaften er auf Basis seiner eigenen Interpretation dessen, was in dem betreffenden Diskurs und der Textsorte angemessen ist, in den Vordergrund rücken will. Roz Ivanič bringt dies in der Einleitung zu ihrem Buch „Writing and Identity“ (Ivanič 1998) deutlich zum Ausdruck:

I am a writer with a multiple social identity, tracing a path between competing ideologies and their associated discourses. I have an idea of the sort of person I want to appear in the pages of this book: responsible, imaginative, insightful, rigorous, committed to making my research relevant to adults who return to study. At any rate that is the sort of person I think I want to be as a member of the academic discourse community. I would want to appear responsible, imaginative, insightful, rigorous and committed in most of my social roles, but not all. For example, I'm not sure how important it is to me in my role as a mother to be rigorous. There are also parts of my identity as a mother which I don't think I portray in my academic writing, such as being loving. (Ivanič 1998; 1 ) 
Vorhergehende Untersuchungen (s. Kap. 3.3.2 haben gezeigt, dass im wissenschaftlichen Schreiben vor allem die Rolle des Verfassers (Bsp. 1) und 2), d. h. der Person, die den Text strukturiert und die Leser anleitet, und die des Forschers (Bsp. 3 und 4), der bestimmte forschungsrelevante Handlungen vollführt hat, mit der ersten Person realisiert werden:

(1) As I document more fully below, other former state socialist countries-particularly those not yet studied by stratification researchers-experience widespread, sustained arrears. (Gerber 2006)

(2) Ich möchte die Unterschiede kurz skizzieren. (Albert 2005)

(3) I interviewed 57 key informants (in 54 separate interviews), with roughly half of the interviews devoted to each of the two cases. (Bartley 2007)

(4) In den multivariaten Analysen verwende ich eine binäre Variable, die erfasst, ob die befragte Person in einer der genannten Organisationen aktiv ist. (Strauß 2009)

Dies ist damit erklärbar, dass ein Text immer einen Produzenten, in diesem Fall den Autor, voraussetzt und bei einem wissenschaftlichen Text dieser Produzent per Definition ein Wissenschaftler bzw. eine Wissenschaftlerin sein sollte. Diese grundsätzliche und eigentlich triviale Einteilung bietet aber doch noch einige Möglichkeiten zur Diversifikation dieser beiden Rollen, wie im Folgenden gezeigt wird.

Hier wird untersucht, wie diese beiden Rollen in den deutschen und US-amerikanischen Texten realisiert werden, welche sinnvollen Unterkategorien oder evtl. noch andere wichtige Rollen es gibt und insbesondere, ob und wenn ja, wie sich diese Rollen und ihre sprachliche Darstellung zwischen den deutschen und den US-amerikanischen Texten unterscheiden.

Dabei muss allerdings berücksichtigt werden, dass die Rollen Wissenschaftler und Autor fließend sind und ineinander übergehen können bzw. dass verschiedene sprachliche Mittel miteinander kombiniert werden können, so dass in einigen Fällen nicht eindeutig entscheidbar ist, welche der beiden Rollen im Vordergrund steht 1 Es ist daher sinnvoll, diese Rollen als Kontinuum zu betrachten, das die verschiedenen Handlungsmöglichkeiten, die einer Person im Rahmen einer wissenschaftlichen Forschung von der Datenerhebung bis zur Veröffentlichung der Ergebnisse zur Verfügung stehen, widerspiegelt.

Im Folgenden werden die Ergebnisse der Korpusanalyse zu den Subjekten in der ersten Person Singular und Plural sowie zum unpersönlichen MAN vorgestellt. Zunächst wird überprüft, inwieweit sich die im Theorieteil (s. Kap. 3.3.2) vorgestellten Kategorien in den hier untersuchten Texten wiederfinden lassen. Danach werden die

\footnotetext{
${ }^{1}$ In diesen Fällen wurden die Beispiele der Rolle zugeordnet, die über das Verb transportiert wird.
} 
Ergebnisse des deutschen und des englischen Teilkorpus verglichen und kulturelle Unterschiede in der Selbstdarstellung anhand von Pronomen diskutiert.

\subsection{Beschreibung der Rollen}

\subsubsection{Person Singular}

Die erste grundlegende Fragestellung, die in dieser Arbeit untersucht werden soll, ist, wie die erste Person Singular in den untersuchten Texten verwendet wird, d. h. welche sozialen Rollen, die sich die Autoren in ihren Texten zuschreiben, damit realisiert werden. Dabei gehe ich im Folgenden von den in Kap. 3.3.2 vorgestellten übergeordneten Rollen Forscher und Verfasser aus und gliedere als Unterkategorien, welche Handlungen die Autoren sich über das ICH zuschreiben. Ich beginne hier mit der Beschreibung der Kategorien, die sich bei der Analyse der beiden Teilkorpora ergeben haben und stelle diese Kategorien zunächst anhand von Beispielen vor.

Basierend auf den in Kap. 3.3.2 vorgestellten Ergebnissen aus anderen Studien habe ich die Daten zunächst grob in die Kategorien Forscher und Verfasser eingeteilt. Sämtliche Vorkommnisse, bei denen keine dieser beiden Rollen im Vordergrund steht, werden in die Kategorie Andere eingeordnet.

\subsubsection{Verfasser}

Unter der Rolle Verfasser werden alle versprachlichten Handlungen und Prozesse zusammengefasst, die als textintern gewertet werden können. Dies sind diejenigen Handlungen, die direkt mit dem Erstellen des Textes verbunden sind, die ihn strukturieren oder den Leser durch den Text führen. Im Gegensatz dazu finden die ForscherHandlungen außerhalb des Textproduktionsprozesses statt und können beispielsweise auch als Grundlage für andere Texte oder Präsentationen genutzt werden (z. B. Handlungen im Rahmen der Datenerhebung oder -analyse etc., s. Kap. 5.1.1.2, S. 104.

Die relevanten Verfasserhandlungen umfassen vor allem verbale oder graphische Darstellungen sowie die Prozesse der Lesersteuerung (I as the guide) und Textstrukturierung (I as the architect) (Tang und John 1999. 27-28) bzw. der Textkommentierung (vgl. z. B. Graefen 2000; Fløttum u. a. 2006; Steinhoff 2007). Entsprechend werden hier viele für wissenschaftliche Texte obligatorische Handlungen ausgeführt, wie z. B. das Darstellen des im Text verfolgten Fokus bzw. der Ziele, des theoretischen Hintergrundes, das Diskutieren und Definieren wesentlicher Terminologie etc. Dabei ist zu beachten, dass diese Handlungen nicht als Sprechhandlungen betrachtet werden, auch wenn einige der hier beschriebenen Handlungen als Sprechhandlungen gelten könnten. Es geht an dieser Stelle vielmehr um die Handlung, die das Verb denotativ benennt und über die der Autor des Textes eine bestimmte soziale Rolle einnimmt. 
Außerdem muss auch immer mit berücksichtigt werden, dass die Rolle Verfasser Teil der übergreifenden wissenschaftlichen Tätigkeit eines Wissenschaftlers ist und auch die Selbstdarstellung als Verfasser dazu dient, eine wissenschaftliche Persona oder Identität zu konstruieren.

\subsection{Verbale und graphische Darstellungen}

Die erste Verfasserkategorie enthält Handlungen der verbalen und graphischen Darstellung von Daten, Ergebnissen etc. Diese werden von Verben wie describe, illustrate oder present umgesetzt ${ }^{2}$. Der Verfasser bereitet Informationen auf und präsentiert sie dem Leser, wobei er die aktive und verantwortliche Person ist, die die Handlungen ausführt. Der Leser ist in den meisten Fällen nur Zuhörer bzw. Zuschauer und wird darüber informiert, was der Verfasser tut bzw. getan hat.

(5) Before exploring possible reasons for this lack of organizing, particularly nonNLRB organizing, I discuss the respective success rates of the two repertoires. (Martin 2008)

(6) To enrich the debate, I present a targeted set of new analyses of MTO data, along with original data from a longitudinal observational study that complements in time and space one of the MTO sites, allowing direct comparison of patterns of residential movement. (Sampson 2008)

(7) Diesbezügliche Stärken und Schwächen von Lebensstiltypologien möchte ich an einem Anwendungsbeispiel illustrieren, das verschiedentlich als vorbildlich für seine Leistungsfähigkeit erachtet wird. (Otte 2005)

(8) Ich möchte die Unterschiede kurz skizzieren. (Albert 2005)

Die wichtigsten Verben in dieser Kategorie sind z. B. skizzieren/eine Skizze vorlegen, illustrieren, discuss, present, show. Nur in wenigen Fällen werden hier Verben benutzt, die dem Leser etwas mehr Verantwortung geben und ihm die Entscheidung zugestehen, ob er die dargestellten Informationen so akzeptiert, z. B. offer, propose.

(9) I offer three dimensions of transnational political opportunity structures that global governance institutions affect in order to constitute power transnationally. (Kay 2005)

(10) In this article, I propose an analytic approach to political contention in state socialism that presumes neither perfect quiescence nor perpetual resistance on the part of most citizens. (Straughn 2005)

\subsection{Darstellen des Ziels bzw. des Fokus des Textes}

Anhand der hierunter gefassten Handlungen zeigt der Verfasser den spezifischen Fokus und das Ziel des Textes auf und teilt sie dem Leser mit, um so dessen Verständnis

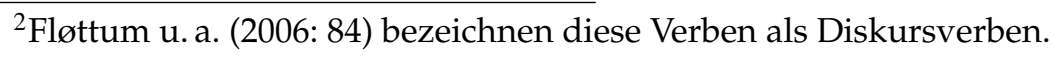


des Textes zu erleichtern. Hier besteht ein Unterschied zu der Forscher-Handlung des „Fokussierens der Forschungsfrage“ (s. Kap. 5.1.1.2.1), da diese natürlich den gesamten Forschungsablauf bestimmt und damit außerhalb der reinen Texterstellung wirkt. Dahingegen ist das hier beschriebene „Darstellen des Ziels bzw. Fokus des Textes“ deutlich enger auf den Text ausgerichtet und kann sich potenziell von den Zielen und Fokussen anderer Präsentationen der gleichen Forschung unterscheiden. Dies wird an den Beispielen vorrangig daran deutlich, dass im weiteren Kotext Verweise auftreten, die diese Handlung eindeutig im Rahmen des jeweils vorliegenden Textes verorten, z. B. "for the purposes of this article“ (Beisp. 11), „im Folgenden“ (Beisp. 12).

(11) Instead of a complete or definitive history of viaticals and life settlements, for the purposes of this article I focus on elements of the development of the market that inform and are informed by a sociological analysis of culture and morality in the marketplace. (Quinn 2008)

(12) Ich konzentriere mich im Folgenden allein auf die Darstellung und Interpretation der logistischen Regressionsanalysen. (Gerhards 2009)

Diese Beschränkung auf den Textzusammenhang wird insbesondere in Beispiel 11 deutlich. Hier zeigt der Autor selber eine alternative Zielsetzung auf, die er ggf. hätte verfolgen können, die er allerdings hier nicht verfolgt. Und auch Beispiel[12 impliziert, dass die Daten und deren Auswertungsmöglichkeiten, die der Autor zur Verfügung hatte, auch andere Darstellungsweisen und -perspektiven erlaubt hätten, dass er aber für diesen Text(-teil) bewusst die Analyse der logistischen Regression ausgewählt hat.

\subsection{Theoretischer Hintergrund}

Eine weitere obligatorische Handlung für wissenschaftliche Autoren eines Artikels ist das Darstellen des theoretischen Hintergrundes 3 , auf den sie sich in ihrer Arbeit beziehen. Dieser Hintergrund kann mit unterschiedlichen Skopen dargestellt werden. Die Beispiele hier beziehen sich wie oben auch konkret auf den jeweiligen Text. Parallel dazu werden unter der Forscher-Rolle nochmals Handlungen erfasst, die den theoretischen Hintergrund auf eine Weise präsentieren, die deutlich macht, dass dieser Hintergrund über den einzelnen Text hinaus für die gesamte Forschung relevant ist (s. Kap 5.1.1.2.2, S. 107).

(13) In the following section, I situate my work in the current research on the role of education in social stratification. (Grodsky 2007)

(14) Although indebted to this scholarship, in this article I engage mostly with works that have tried to describe, interpret, and explain forms of the American religion prevalent in the past 50 years. (Madsen 2009)

\footnotetext{
${ }^{3}$ Diese Kategorie wird in der weiteren Auswertung zusammengefasst mit der Kategorie Darstellen des Ziels bzw. des Fokus des Textes betrachtet.
} 
(15) For brevity and clarity, I focus the discussion around the work of Swidler (1986, 2001), undoubtedly the most well-known and exemplary member of the "repertoire" school among sociologists of culture. (Vaisey 2009)

\subsection{Terminologieklärung}

Das Klären und Definieren von Begriffen ist ebenfalls eine Handlung, die in der Textsorte des wissenschaftlichen Artikels praktisch obligatorisch ist. Hier werden vorrangig im fachlichen Diskurs bereits vorhandene Begriffe spezifiziert, d. h. die vom Autor verwendete Definition wird dem Leser mitgeteilt und der Skopus dieser Terminologie wird im Kotext häufig durch einen entsprechenden Zusatz auf den jeweiligen Artikel eingeschränkt. Diese Handlungen werden hier auch wieder abgegrenzt vor der verwandten, aber eher als Forscherhandlung einzustufenden Handlung der Begriffsbildung, durch die neue Begriffe geprägt und in den Diskurs eingeführt werden (s. Kap 5.1.1.2.7, S. 110).

(16) In this article, I reserve the term "certification" for external, third-party systems that include some rationalized procedure for assessing compliance, rather than applying it to more ad hoc claims making. (Bartley 2007)

(17) Therefore, to emphasize movement actions, rather than structure, throughout the article I refer to the two organizing strategies as "repertoires", or the "whole set of means" a social movement has at its disposal to achieve new gains [...]. (Martin 2008)

(18) Ich bezeichne hier mit Kultur den gesellschaftlichen Bereich, der sich auf Kunstaktivitäten im weitesten Sinne bezieht. (Gerhards 2009)

In den obigen Beispielen wird dabei durch den Zusatz in this article (Beisp. 16), throughout the article (Beisp. 17) und hier (Beisp. 18) deutlich, dass sich die Klärung der Begriffe auf den jeweils vorliegenden Artikel bezieht. Das heißt natürlich nicht notwendigerweise, dass die Autoren diese Definition nicht auch anderweitig so verwenden, hier kommt es ihnen aber offensichtlich darauf an, dass der Begriff im Rahmen des konkreten Textes bestimmt wird.

\subsection{Textstrukturierung}

Eine weitere Handlung, die der Verfasser eines wissenschaftlichen Textes vollführen muss, ist das Strukturieren des Textes. Dies dient $\mathrm{u}$. a. ebenfalls dazu, dem Leser die Orientierung und das Verständnis zu erleichtern. Hier stellt sich der Verfasser entsprechend als derjenige dar, der den Text strukturiert, über die inhaltliche Abfolge bestimmt (Beisp. 19) und der sich (und den Leser) innerhalb dieser Abfolge verortet (Beisp. 20 und 21). Diese Handlungen schließen auch Vor- und Rückverweise mit ein (Beisp.21 und 22). 
(19) I have divided this article into six principal sections. (Schrank 2010)

(20) I conclude with a summary of my historical findings and discuss more general implications of a legal framing perspective for theory and research on cultural framing processes and social movements. (Pedriana 2006)

(21) Zum Abschluss möchte ich noch einmal zu Max Webers Charisma-Theorie zurückkehren, um den Legitimationszugewinn, der mit den Selig- und Heiligsprechungen verbunden ist, durch eine erweiterte Theorie des Charismas zu erklären und damit das herrschaftssoziologische "Geheimnis" der Selig- und Heiligsprechungen ein wenig zu lüften. (Bienfait 2006)

(22) Ich komme auf das für die Fragestellung des Artikels wichtige Verhältnis zwischen inkorporiertem kulturellem Kapital, Geschmack, Habitus, Kunstorientierung und Lebensstilen gleich nochmals genauer zurück. (Gerhards 2009)

\subsection{Sonstige Metakommunikation}

Hierunter fallen alle weiteren Handlungen, mit denen der Autor aus seinem Text heraustritt und seine Leser über den Text und seine Inhalte informiert (z. B. Beispiel 23). Diese Kategorie enthält vor allem Bemerkungen darüber, was in diesem Text nicht aufgegriffen wird, was der Autor explizit nicht verfolgt oder darstellen will (Beispiele 24. 25 und 26).

(23) Offensichtlich habe ich diesen Begriff an genannter Stelle aber eingeführt, um etwas grundlegend anderes zu bezeichnen. (Albert 2005)

(24) Such a typology is, in fact, implied by the results, but I refrain from complicating table 1 with a cross-classification. (Gibson 2005)

(25) It is an open question, and one I do not take up here, of whether we are now moving into some third stage, characterized by the declining legitimacy of the modern state with a concomitant dissolution of the state's symbolic power. (Loveman 2005)

(26) Da sich unsere Daten aber allein auf eine Hochkulturorientierung beziehen, die so genannte „Allesfresserthese“ aber unterschiedliche Kulturaktivitäten (Hochund Populärkultur) einschließt, lasse ich diese These hier unberücksichtigt. (Gerhards 2009)

\subsubsection{Forscher}

Unter die Forscher-Rolle werden hier alle Handlungen gefasst, die eine im engeren Sinne „wissenschaftliche“ Tätigkeit umfassen, d. h. also alle Tätigkeiten, die nicht direkt und ausschließlich den Schreibprozess betreffen oder die Metakommunikation mit dem Leser beinhalten. Grundsätzlich werden also als „wissenschaftliche“ Handlungen hier diejenigen aufgefasst, die textextern ausgeführt wurden und entsprechend 
ggf. auch in anderen Texten oder Präsentationsformen wiedergegeben werden können. Textinterne Handlungen, die den Verfasser charakterisieren, sind demgegenüber an den vorliegenden Text gebunden.

Forschungshandlungen schließen insbesondere solche (textexternen) Handlungen ein, die im Verlauf des Forschungsprozesses, auf dem die untersuchten Texte beruhen, angefallen sind. Dies umfasst

- das Festlegen des Forschungsfokus und der Fragestellung als Basis für die Datenauswahl, -erhebung und -analyse,

- das Festlegen der Ausgangsannahmen und der theoretischen Grundpositionen,

- die Beschreibung der Datenerhebung, inklusive Begründungen für mögliche Eingrenzungen der Daten wie z. B. die Auswahl eines Erhebungsraums etc.,

- die Beschreibung und Diskussion der methodischen Ausrichtung der Forschung,

- die Aufbereitung und Analyse der Daten,

- die Darstellung der Analyseergebnisse.

Darüber hinaus fallen in Anlehnung an vorhergehende Studien (s. Kap. 3.3.2) hierunter auch weiterführende Interpretationen und Argumentationen, insbesondere die Darstellung eigener Gedanken und Ergebnisse, die das vorhandene Wissen in der Community erweitern. Außerdem werden dieser Kategorie auch diejenigen Handlungen zugeordnet, mit denen sich der Autor mit Bezug zu anderen Personen in der wissenschaftlichen Gemeinschaft positioniert, vorrangig über

- das Argumentieren für den eigenen Standpunkt inklusive der Interpretation der eigenen Ergebnisse,

- das Hervorbringen eigener Ideen und eigenen Wissens, die in die Gemeinschaft getragen werden; hierzu zählt auch die Begriffsbildung, also das Einbringen neuer Begriffe in den Wissensstand der Community $\left.\right|^{4}$ und

- die Danksagung ${ }^{5}$ mit der neben dem Dank an ausgewählte Personen auch eine Einordnung in die Gemeinschaft und eine Positionierung mit Bezug auf diese (ggf. deutlich etablierten) Personen erfolgt.

\subsection{Fokussieren der Forschungsfrage}

Hierunter werden alle Handlungen zusammengefasst, mit denen die Autoren den Fokus darlegen, unter dem sie die Forschung durchgeführt haben. Dies ist nicht zu verwechseln mit der Fragestellung des Textes, deren Angabe textintern und damit als Er-

\footnotetext{
${ }^{4}$ Dies ist nicht zu verwechseln mit dem Definieren der im Text verwendeten Begriffe, was eher in den Bereich des Verfassers fällt. Hier wird deutlich, dass diese Kategorien, wie so häufig, nicht immer eindeutig trennbar sind und natürlich auch einzelne Handlungen nicht immer zweifelsfrei eingeordnet werden können. Im Großen und Ganzen haben sich die unklaren Fälle aber in Grenzen gehalten.

${ }^{5}$ Danksagungen sind genau genommen ambivalent zu betrachten, da sie einerseits metakommunikative Funktion haben (vgl. z. B. Steinhoff 2007. 190) und damit als Ausprägung der Verfasser-Rolle gewertet werden können. Da hier jedoch die Wirkung, die die untersuchten Handlungen auf die Positionierung der Autoren in der Gemeinschaft haben, im Vordergrund steht, ist es in diesem Zusammenhang sinnvoller, sie als Unterpunkt der Forscher-Handlungen zu betrachten.
} 
füllen der Autorenrolle zu werten ist (s. Kap. 5.1.1.1.2). Der Forschungsfokus bezieht sich demgegenüber auf das gesamte Forschungsprojekt bzw. den Teilbereich, in dem der jeweilige Autor arbeitet. In diese Kategorie fallen auch diejenigen Handlungen, in denen Forschungsziele expliziert werden:

(27) I consider how, conceptually, arrears can play an independent stratifying role; that is, how inequality in getting paid might differ from inequality in contracted wages. (Gerber 2006)

(28) Because my methodological approach differs from that used in these influential social scientific studies, however, I emphasize certain dimensions of religious individualism that they have not clearly noticed. (Madsen 2009)

(29) Ich sehe in theoretisch-konzeptioneller Hinsicht insgesamt das Differenzierungsund Integrationserfordernis, die Verschränktheit von institutionellen Faktoren auf der nationalen Ebene einerseits und branchenspezifischen Veränderungsdynamiken andererseits zu identifizieren. (Teipe 2009)

In diesen Beispielen wird die übergeordnete Fragestellung bzw. das Forschungsdesiderat erläutert, das die konzeptuelle Grundlage für die im Text dargestellte Untersuchung bildet.

\subsection{Auswählen von Hypothesen, Ausgangsannahmen und theoretischen Grundpo- sitionen}

Eng verwandt mit der „Fokussierung der Forschungsfrage“ ist auch das „Auswählen von Hypothesen, Grundannahmen und theoretischen Hintergründen". Auch diese Tätigkeiten erfolgen normalerweise vor oder ggf. während der Untersuchung und insofern deutlich vor dem Verfassen eines wissenschaftlichen Artikels, auch wenn die Umsetzung im Präsens in den hier genannten Beispielen den Eindruck erzeugt, diese Handlung würde im Prozess des Schreibens ausgeführt 6

In Beispiel 30 wird eine grundlegende Hypothese darüber formuliert, wie sich die untersuchten Variablen zueinander verhalten, ebenso wie in Beispiel 31. In Beispiel 32 stellt der Autor eine Grundannahme vor, auf der die Operationalisierung seiner Variablen basiert.

(30) I assume that community colleges have no preferences for the types of students whom they enroll; $\beta_{b}=0$ and $\gamma_{b}=0$. (Grodsky 2007)

(31) I thus hypothesize that the association between black population size and hate crime prosecution is positive until the black population reaches $20 \%$, at which point a negative association emerges. (King 2008)

\footnotetext{
${ }^{6}$ Auch wenn es möglicherweise Fragestellungen gibt, die nur in Hinblick auf eine spezifische Veröffentlichung untersucht werden, sind diese Handlungen dennoch als textextern zu betrachten, da sie im Rahmen der vorauszusetzenden Forschungslogik die gesamte Untersuchung betreffen.
} 
(32) Ich gehe davon aus, dass die Höhe des Anteils der Erwerbspersonen im Kultursektor eine einigermaßen geeignete Größe ist, um die hochkulturelle Infrastruktur eines Landes zu operationalisieren. (Gerhards 2009)

In den folgenden drei Beispielen werden die theoretischen Ausgangspositionen dargestellt. In Beispiel 33 verknüpft der Autor verschiedene theoretische Positionen, um daraus seine eigene Basis zu konstruieren. Beispiel 34 zeigt, wie der Autor zunächst Schwächen in bestehenden Arbeiten hervorhebt, um so seinen eigenen Ansatz und damit seine eigene Position zu stärken. Und auch in Beispiel 35 wird der theoretische Unterbau der Untersuchung erläutert, wobei der Autor deutlich hervorhebt, dass sich dieser Unterbau auf die empirischen Analysen und damit auf die gesamte Forschung und nicht nur auf den vorliegenden Text beziehen (vgl. die entsprechenden Handlungen in der Verfasser-Rolle, S. 102.

(33) I link tenets of neoinstitutional theory with extant work on criminal punishment to suggest facets of the local legal environment that are conducive to organizational decoupling. (King 2008)

(34) To address this theoretical shortcoming, I integrate a key strand of law and society scholarship that focuses on how law's deeply embedded "constitutive" symbols and categories influence cultural framing processes. (Pedriana 2006)

(35) Für den Kontext meiner empirischen Analysen sind allein die Arbeiten, die sich auf die Rezipientenseite beziehen, von Relevanz; und hier orientiere ich mich in erster Linie an der klassischen Arbeit „Die feinen Unterschiede“. (Gerhards 2009)

\subsection{Daten erheben}

Ein weiterer Bereich, in dem sich die Autoren der untersuchten Texte als agierende Personen darstellen, ist die Auswahl der passenden Methode und die Durchführung der Datenerhebung. Obwohl diese Kategorie die zweitgrößte für die amerikanischen Texte ist, enthält sie keine deutschen Beispiele.

(36) I utilize a comparative case study approach, examining two fields - apparel and forest products - and paying close attention to the unfolding of events in each. (Bartley 2007)

(37) I constructed such a data set from cross-sectional data on the states that I collected from a wide range of published and unpublished sources, including government reports, private publications, and archival records. (Chen 2007)

(38) I personally conducted around 35 in-depth interviews with respondents over a period of more than two years. (Vaisey 2009) 
(39) I restrict the institutional samples to community colleges, junior colleges, and comprehensive public and private not-for-profit colleges and universities. (Grodsky 2007)

In Beispiel 36 stellt der Autor seinen empirischen Zugang vor, wobei er im zweiten Teil seine eigene Aktivität und vor allem Sorgfalt („paying close attention“) deutlich hervorhebt. Die folgenden Beispiele stellen die Autoren als die aktiven Personen bei der Datenerhebung dar. In Beispiel 37 identifiziert sich der Autor einerseits eher aus einer abstrakten Perspektive als die Person, die einen Datensatz konstruiert, um dann konkreter darauf einzugehen, welche Art Daten er gesammelt hat, wodurch der Leser eine lebensnahe Vorstellung von dem mühevollen Prozess der Datenerhebung erhält. In Beispiel 38 hebt der Autor zusätzlich zur Selbstreferenz über die erste Person anhand des „personal“ noch stärker hervor, dass er die von ihm untersuchten Interviews selber durchgeführt hat, wobei auch hier die Angabe des langen Zeitraums, abgesehen von der notwendigen Transparenz, auch noch einmal den Arbeitsaufwand hervorhebt, den der Autor persönlich in die Datenerhebung investiert hat. Beispiel 39 präsentiert den Autor als Entscheider, der die Datenauswahl festlegt.

\subsection{Daten aufbereiten und analysieren}

Neben der Datenerhebung wird auch bei der Aufbereitung und der Analyse der Daten häufig die Person des Autors in den Fokus gestellt und über die erste Person Singular direkt als Ausführender benannt.

(40) In other instances, I used the data to generate time-constant variables. (Chen 2007)

(41) I categorize unions that meet at least three of these criteria as having a partially developed relationship. (Kay 2005)

(42) In den multivariaten Analysen verwende ich eine binäre Variable, die erfasst, ob die befragte Person in einer der genannten Organisationen aktiv ist. (Strauß 2009)

Hier handeln die jeweiligen Autoren einerseits im Rahmen der Datenaufbereitung (Beispiel 40), andererseits sind sie explizit diejenigen, die die Analyse durchführen (Beispiele 41 und 42) und sich dadurch aktiv innerhalb ihres Forschungsprozesses darstellen.

\subsection{Ergebnisse erzielen}

Der letzte Handlungskomplex, der im engeren Sinne zum Forschungsprozess gehört, ist die Darstellung der erzielten Ergebnisse. Dies wird im Englischen sehr häufig über das Verb to find realisiert (Beispiel 43) 7 , welches auch gelegentlich in einer Vergan-

\footnotetext{
${ }^{7}$ Allerdings wird das englische find auch gelegentlich in seiner anderen Bedeutung als Meinungsangabe benutzt, vergleichbar mit dem deutschen finden: „Ludwig et al. themselves argue that it is unfor-
} 
genheitsform verwendet wird. In diesen Fällen wird es besonders deutlich, dass diese Handlungen außerhalb des Textes stehen, da sie so zeitlich eindeutig vor dem Schreibprozess stehen (s. Beispiel 44). In jedem Fall aber stellt sich der Autor als die handelnde Instanz dar, die die Ergebnisse und Schlussfolgerungen produziert (Beispiel 45).

(43) As for control variables, I find, most generally, an increase in the size of supermarket establishments examined over the period between 1983 and 1998. (Skaggs 2008)

(44) I also examined change from 1995 to 2002 in the measures of neighborhood social processes noted above (e.g., cohesion and control), and I found that no change trajectory differed significantly by treatment group. (Sampson 2008)

(45) Ähnlich wie Hermann (2004) und Meyer (2001) komme ich zu dem Schluss, dass von einer "Autonomie des Lebensstils" nicht gesprochen werden kann: Mit wenigen Sozialstrukturvariablen lassen sich zwischen 10 und 25 Prozent der Variation in der Lebensstiltypenzugehörigkeit erklären. (Otte 2005)

\subsection{Argumentieren für den eigenen Standpunkt}

Beim „Argumentieren für den eigenen Standpunkt" werden diejenigen Handlungen vollführt, durch die der wissenschaftliche Autor seine eigene Perspektive und Meinung wiedergibt. So stellt er seinen eigenen Standpunkt dar und positioniert sich inhaltlich, wobei er sich auch auf andere Standpunkte und/oder Personen beziehen kann, was gelegentlich Kritik an diesen einschließt.

(46) Although this sort of market-based approach is quite prominent in the existing literature on private regulation (...), I argue that another approach must also be considered.

(47) I believe that both forms of pressure play a role in shaping employment opportunities.

(48) Yet, I consider the comparative study of different interaction settings to be an important avenue for future research and return to this in the conclusion.

(49) Dennoch möchte ich die Hypothese, dass die gesamtgesellschaftliche Erfahrung der Arbeitslosigkeit auch die Familiengründungsentscheidungen von Personen betrifft, die selbst nicht mit dem Problem konfrontiert sind, zurückweisen, da die entsprechenden Effekte zu schwach sind. (Brose)

\subsection{Aufstellen neuer Claims und neuer Ideen}

Der Originator vertritt neue Ideen und stellt Behauptungen auf, die auf eine Erweiterung des fachlichen Wissens der Community abzielen. Damit setzt er seine eigenen 
Ergebnisse in Bezug mit dem allgemeinen fachlichen Wissen und stellt seinen Beitrag deutlich heraus. Diese Rolle gilt als besonders risikoreich und face-bedrohend, da der Autor sich mit seinen Aussagen sozusagen in die Schusslinie seiner Kollegen stellt bzw. diese ggf. sogar selber angreift, indem er impliziert, dass wichtige Perspektiven bisher (von ihnen) nicht bedacht wurden. Diese Rolle wurde in Vergleichen zwischen studentischen und wissenschaftlichen Autoren entsprechend auch immer mit fortgeschrittenen Schreiberfahrungen in Zusammenhang gebracht (vgl z. B. Hyland 2002a).

(50) I add to existing explanations the critical role of refugee camps as organizational sites of mobilization for many women guerrillas. (Viterna 2006)

(51) I intend thereby to contribute to the growing body of recent research that recognizes symbolic interaction and social performance as constitutive features of political contestation [...]. (Straughn 2005)

In diese Kategorie fällt auch die Begriffsbildung, die, anders als das Klären der Terminologie (Kap. 5.1.1.1.4), nicht zu den textinternen Handlungen gezählt werden darf. Hier geht es vielmehr darum, dass im Rahmen des Forschungsprozesses Begriffe spezifisch neu benutzt bzw. sogar ganz neue Begrifflichkeiten entwickelt werden. Darauf, dass dies eine „wissenschaftliche“ Handlung im hier vertretenen Sinne ist, die auch ein hohes Risiko birgt, hat auch schon Meyers hingewiesen:

To offer a new term for a phenomenon discovered or created by the discipline is to invite the question 'Who are you to name this stuff?'. (Myers 1989: 6, Hervorhebung im Original)

Auf dieses Risiko wird entsprechend gelegentlich auch mit einer Abschwächung des Claims durch ein Modalverb reagiert (Beispiel 53).

(52) I call this process the "political construction of market institutions".

(53) Thus, I would like to use the term "liminal legality" to express the temporariness of this condition, which for many Central Americans has extended indefinitely and has come to define their legal position. (Menjívar 2006)

(54) Diese Art und Weise der Handlungszuschreibung bezeichne ich als den Modus der Zuschreibung auf Gründe [...]. (Schulz-Schaeffer 2009)

\subsection{Danksagungen}

Danksagungen werden hier zur Wissenschaftlerrolle gezählt, obwohl sie beispielsweise von Steinhoff (2007; 180) zum Verfasser-Ich gezählt wurden. Steinhoff schreibt Danksagungen vor allem metakommunkative Funktionen zu, indem der Schreibende sich den Personen gegenüber, die ihn unterstützt haben, erkenntlich zeige (Steinhoff 2007; 180). Weiterhin weist er jedoch darauf hin, dass die Danksagung auch dazu diene, allen 
Lesern zu kommunizieren, „welcher scientific community der Text zuzurechnen ist" (Steinhoff 2007: 180).

Während Steinhoff diese identifizierende und positionierende Funktion aber nur als Nebenfunktion „zwischen den Zeilen“ versteht (Steinhoff 2007; 180), liegt hier das Augenmerk genau darauf. Durch die Nennung bestimmter Personen („Namedropping") kann eine Assoziation mit diesen Personen, ihren wissenschaftlichen Erfolgen und dadurch ihrem Status hervorgerufen werden. Ähnliches gilt auch für die Nennung von Institutionen der Forschungsförderung wie z. B. der Deutschen Forschungsgemeinschaft (DFG). Weiterhin sind Danksagungen auch im Rahmen des allgemeinen wissenschaftlichen Qualitätskriteriums der Transparenz zu betrachten, da sie mögliche Einflüsse und Kooperationen offenlegen, sowie „beispielhaft erscheinen“ (s. Kap. 5.4. vgl. auch Mummendey u.a.(1995: 148)).

(55) For comments and suggestions, I thank Elizabeth Armstrong, (... $)_{8}^{8}$, and the AJS reviewers. (Bartley 2007)

(56) I thank John Levi Martin for bringing this episode to my attention. (Gerber 2006)

(57) Ich danke Martin Kroh, (...) sowie einem anonymen Gutachter und den Herausgebern für hilfreiche Anregungen und Kommentare. (Isengard 2005)

(58) Für Anregungen und Kritik danke ich Oliver Arránz-Becker, (...) und zwei anonymen Gutachtern. (Lois 2008)

Diese eigene direkte Zuordnung zu einer bestimmten scientific community macht deutlich, dass der Schreibende sich hier im Rahmen dieser Gemeinschaft als Wissenschaftler positionieren will, was selbstverständlich auch außerhalb des jeweiligen Textes wirken soll und wodurch er sich eindeutig als wissenschaftlich handelnde Person darstellt.

Danksagungen stellen ein geläufiges, aber nicht obligatorisches Teilsegment der Textsorte wissenschaftlicher Fachartikel dar. Im Gegensatz zu den anderen Abschnitten eines Artikels, in welchen der Autor normalerweise den Anschein erwecken möchte, ein unabhängiger, unpersönlicher, rein rationaler Wissensvermittler zu sein, kommen in Danksagungen die persönlichen, menschlichen und privaten Seiten des Wissenschaftsbetriebs deutlich zum Vorschein. [...] Dadurch machen sie, mehr als alle anderen Teilsegmente dieser Textsorte, die komplexen Netzwerke persönlicher bzw. interpersoneller Dankesschuld sichtbar, die dem dialogischen Prozess der Wissenschaft zugrunde liegen. (Sanderson (2005; 66) mit Bezug auf Giannoni (2002, 9))

\footnotetext{
${ }^{8}$ Für die Analyse irrelevante Informationen wie längere Aufzählungen z. B. von Namen, Literaturangaben etc. werden in den Beispielen nicht wiedergegeben.
} 
Durch die Danksagung wird der interaktionale Charakter des wissenschaftlichen Artikels also besonders deutlich (Hyland 2002a: 1106).

\subsubsection{Andere}

In dieser Kategorie werden alle versprachlichten Handlungen zusammengefasst, die zwar über den Text hinaus reichen, aber nicht direkt als „wissenschaftliche“ Handlungen im engeren Sinne zu verstehen sind. Diese werden überwiegend in Kombination mit dem repräsentativen ICH gebraucht, mit dem sich der Autor als Repräsentant einer bestimmten Gruppe darstellt (der er aber nicht notwendigerweise angehören muss). Dieser ICH-Gebrauch ist daher am ehesten mit dem "Gemeinschafts-WIR“ (s. S. 119) oder "Gemeinschafts-MAN" (s. S. 129) zu vergleichen, in denen das WIR bzw. MAN auch eine ganze Gruppe bzw. sogar die gesamte Menschheit bezeichnet.

(59) One is the logical-sequential nature of Pshifts, which make it impossible, for instance, for someone to turn usurp after I am addressed if I am never addressed. (Gibson 2005)

(60) Wenn mir beispielsweise ein Tischnachbar den Brotkorb hinhält, werde ich normalerweise nicht extra nachfragen, ob er damit die Absicht verfolgt, mir das Brot zu reichen. (Schulz-Schaeffer 2009)

(61) I might use a voucher to move to a lower-poverty neighborhood than where a control group member lives, for example, but that neighborhood may be on a downward trajectory (e.g., with declining house values) while the control neighborhood is stably poor. (Sampson 2008)

Entsprechend steht in Beispiel 59 das I zwar für den Autor, dieser aber ist ein Beispiel für alle Personen die jemals im Rahmen eines Gesprächs angesprochen (oder gerade nicht angesprochen) werden. Hier hätte man, zumindest im Deutschen, eventuell sogar eher ein MAN erwarten können. Und auch das Beispiel 60 ist entsprechend so zu interpretieren, dass der Autor hier zwar von sich selber spricht, aber davon ausgeht, dass allen anderen Menschen der entsprechenden Sprach- und Kulturgruppe die jeweiligen Situationen genauso interpretieren würden, wie er es tut.

Beispiel 61 unterscheidet sich von den anderen beiden Beispielen insofern, als dass das ICH sich hier offenbar nicht (nur) auf den Autor bezieht. Es ist sogar sehr unwahrscheinlich, dass er jemals in die beschriebene Situation kommen wird. Dennoch benutzt er hier das ICH und stellt dadurch eine besondere Nähe zu den von ihm untersuchten Personen her. Dies könnte möglicherweise daran liegen, dass er sich besonders mit der Personengruppe, die er hier untersucht hat, identifiziert. Dagegen spricht jedoch, dass er sich zumindest in diesem Artikel auf Datenmaterial aus Volkszählungen beruft, eine derart ausgeprägte Identifikation wäre vermutlich eher bei direkteren 
Erhebungsmethoden wie Interviews erwartbar. Insofern könnte diese Form des ICHGebrauchs auch eine individuelle Eigenschaft dieses Autors sein, die er im gleichen Artikel noch einmal in zwei weiteren aufeinanderfolgenden Sätzen (allerdings in einer Fußnote) benutzt:

(62) Suppose I were a complier and moved further south in Chicago (figs. 2 and 5) to satisfy the voucher requirement. If I or my children had friends or family back in the old neighborhood who were voucher eligible, and we complained about the hardship of moving or, to the contrary, expressed enthusiasm about the new neighborhood, these social interactions could have influenced the moving decisions or destinations of those in our network-especially given the multiyear window of randomization (and thus lease-up) and the fact that many compliers drifted back to poor neighborhoods. (Sampson 2008)

Diese Formen des ICH-Gebrauchs sind sicherlich als wenig riskant und face-bedrohend für den Autor zu werten, da er sich hier als typischen Vertreter und Repräsentanten einer Gemeinschaft darstellt. Dies entspricht auch der Interpretation von Tang und John (1999) (s. Kap. 3.3.2.1), die die Repräsentantenrolle in ihrem Korpus nur in studentischen Texten und nur im Plural umgesetzt gefunden haben. Ihrer Interpretation nach machen sich die Autoren in dieser Rolle zu einer "non-identity“, da sie dem Leser eigentlich nichts von sich selbst preisgeben (Tang und John 1999. 27). Dies ist hier ebenfalls der Fall, dennoch ist es sicherlich auf der Beziehungsebene relevant, dass die Autoren hier bewusst darauf verzichten, den Leser offen in diese Gemeinschaft mit einzuschließen und ihm dadurch die Wahl zu lassen, ob er sich ebenfalls zugehörig fühlt und sich somit mit den getroffenen Aussagen identifiziert.

\subsubsection{Person Plural}

Wie schon in Kapitel 3.3.1.2 beschrieben, können von der ersten Person Plural unterschiedliche Personengruppen bezeichnet werden. Im Folgenden werden daher zunächst die unterschiedlichen Referenzmöglichkeiten des WIR vorgestellt. Wegen der intrinsischen Vagheit der ersten Person Plural gibt es hier einige Fälle, die nicht eindeutig einer der untenstehenden Kategorien zugeordnet werden können.

Neben dem schon in anderen Untersuchungen beschriebenen inklusiven und exklusiven Gebrauch von WIR ergab sich aufgrund der besonderen Perspektive dieser Arbeit, die sich nicht mit Sprechhandlungen beschäftigt, sondern mit der sprachlichen Darstellung von Handlungen, die außerhalb des Textes stattgefunden haben, eine weitere Kategorie. Diese wird hier als "pseudo-inklusives WIR“ bezeichnet und ist mit dem von Fløttum u. a. (2006, 99) beschriebenen metonymischen Inclusive 'we' for 'I' vergleichbar. Da das WIR hier in Kombination mit dem Verb(komplex), dessen semantisches Agens und grammatisches Subjekt es ist, interpretiert wird, ergab sich bei eini- 
gen Beispielen eine besondere Kombination an möglichen Interpretationen. In diesen Fällen kann das WIR auf den Leser inklusiv wirken, obwohl die mit ihm assoziierte Handlung den Leser eindeutig nicht mit einschließt.

Dieses WIR spielt in wissenschaftlichen Artikeln insbesondere im Bereich der Hyland'schen Engagement-Strategien eine Rolle (s. z. B. Hyland (2005) und auch Kap. 3.2.2). Es dient dazu, den Leser in die beschriebenen Handlungen mit einzubeziehen und dadurch Entscheidungen, Interpretationen und Argumentationen so darzustellen, als ob der Leser daran beteiligt gewesen wäre. Dadurch kann der Autor den Effekt erzielen, dass die kritische Distanz des Lesers verringert wird und die ggf. risikoreichen und face-bedrohenden Handlungen abgeschwächt werden. Wenn der Leser sich als daran beteiligt wahrnimmt, ist er vermutlich auch eher bereit sie zu akzeptieren und nicht in Frage zu stellen. Fløttum u. a. (2006, 99) vermuten entsprechend, dass dieses WIR die Leser auch emotional beeinflussen kann. Dies ist eine wichtige persuasive Taktik, die sowohl in den deutschen als auch in den englischen Texten angewandt wird.

Zunächst werden hier also die unterschiedlichen Referenzmöglichkeiten des WIR anhand von Beispielen beschrieben. Im Anschluss daran werden dann Beispiele für die Verwendung des WIR mit verschiedenen Autorenrollen gegeben.

\subsubsection{Exklusives WIR}

Das exklusive WIR bezeichnet im Allgemeinen eine Personengruppe, die den Sprecher bzw. den Autor des Textes mit einbezieht, den Hörer bzw. Leser aber nicht. Dabei ist auch hier wieder zu berücksichtigen, dass es nicht um den oder die konkreten Leser geht, sondern letztlich um die vom Autor imaginierte Leserschaft.

Das exklusive WIR lässt sich in zwei Unterkategorien aufteilen, von denen die erste als Äquivalent zum ICH zu werten ist, insofern, als dass dieses WIR die Lesart zulässt, dass es sich ausschließlich auf den Autor beziehen kann. Die zweite Unterkategorie umfasst diejenigen WIR, die eindeutig eine Personengruppe bezeichnen, die neben dem Autor noch mindestens eine weitere Person umfasst, welche auch benannt wird. Der Leser ist aus dieser Gruppe jedoch auf jeden Fall ausgeschlossen.

\subsection{WIR als Autor}

Diese Form des WIR wurde in verschiedenen Untersuchungen (z. B. Kresta|1995: Steinhoff 2007) bisher so interpretiert, dass sie ausschließlich den Autor bzw. die Autoren des betreffenden Textes bezeichnet bzw. bezeichnen kann. Da für die hier untersuchten Korpustexte ohne Ausnahme Einzelautoren angegeben sind, wäre dieses WIR, wenn es denn tatsächlich nur den Autor bezeichnet, als Pluralis Majestatis bzw. Modestiae zu werten. Die Beispiele aus dieser WIR-Kategorie können also so gedeutet werden, dass sie (ausschließlich) auf den Autor referieren. 
Allerdings kann man trotz der Angabe nur eines Autors nicht eindeutig davon ausgehen, dass dieses WIR sich in den Texten auch ausschließlich auf den Autor bezieht. Es können darüber hinaus theoretisch auch immer andere Personen, z. B. Mitglieder der Arbeitsgruppe usw., mit einbezogen sein, die jedoch nicht explizit genannt werden. Dies unterscheidet diese Referenzkategorie von der nachfolgenden „Autor und Andere", in der die weiteren in das WIR eingeschlossenen Personen explizit benannt werden.

(63) Even after we introduced a variety of theoretically motivated covariates that captured largely unstudied aspects of locational attainment-such as depression, criminality, and social support-the substantive picture of our results was unchanged. (Sampson 2008)

(64) We expected this effect on the grounds that feminized occupations in Russia have greater downward wage flexibility and carry greater risks of negative feedback. (Gerber 2006)

(65) Um genauer zu prüfen, ob Erwerbstätige mit einer überproportional hohen Arbeitsbelastung verstärkt zu beruflichen Abstiegsprozessen tendieren, haben wir in einer weiteren Modellspezifikation anhand einer Dummy-Variablen zwischen Personen mit einer Wochenstundenzahl bis zu 42 Stunden und solchen, die 43 Stunden und länger arbeiten, unterschieden (Ergebnisse sind nicht ausgewiesen). (Pollmann-Schuldt 2006)

(66) Die Heiratszahlen, die wir besitzen, sprechen die deutlichste Sprache [...]. (Becker 2005)

In Beispiel 63 bezieht sich das WIR auf die Person(en), die die beschriebene Auswertung vorgenommen und damit die entsprechenden Ergebnisse erzielt hat bzw. haben. Daher kann es die Leser eindeutig nicht mit einschließen. Hier wird auch klar, dass es nicht eindeutig bestimmbar ist, ob der Autor diese Auswertung selber und alleine durchgeführt hat, es ist aber auf jeden Fall vorstellbar. Andererseits ist es genauso möglich, dass er sich auf die Zusammenarbeit mit in diesem Beispiel nicht genannten Kollegen in einer Projektgruppe bezieht.

In Beispiel 64 ist die Situation ähnlich, auch hier ist es zumindest möglich, dass der Autor seine eigenen Erwartungen darstellt und durch den Gebrauch des WIR versucht, seinen Überlegungen eine allgemeinere Gültigkeit zuzuweisen, um so mögliche Kritik an sich bzw. seinen Erwartungen abzuschwächen. Genauso gut ist es aber auch möglich, dass diese Überlegungen in einer Arbeitsgruppe geäußert wurden. Der Ausschluss des Lesers ist hier jedoch eindeutig und auch sprachlich signalisiert, nämlich dadurch, dass das Verb im Simple Past verwendet wird, was zeigt, das die genannte Analyse durchgeführt wurde, bevor überhaupt der Text veröffentlicht wurde.

Ähnliches ist für Beispiel 65 zu sagen. Auch hier ist es eindeutig, dass das WIR den Autor und ggf. seine Kollegen bezeichnet, die die Entscheidungen über die Auswer- 
tungsverfahren der dargestellten Untersuchung treffen. Der Zusatz „Ergebnisse sind nicht ausgewiesen" macht dabei auch deutlich, dass der Leser aus diesem Teil des Entscheidungsprozesses und auch der anschließenden Interpretation ausgeschlossen ist und ihm in den auf dieses Beispiel folgenden Sätzen (die hier nicht wiedergegeben sind) nur die zusammengefassten Endergebnisse dieses Teilbereiches der Auswertung präsentiert werden. In Beispiel 66 wiederum bezieht sich das WIR auf die Person(engruppe), die die Daten besitzt, was den Leser ebenfalls ausschließt.

\subsection{Autor und andere}

Die zweite Variante des exklusiven WIR bezeichnet ebenfalls eine Personengruppe, die zwar den Autor, aber nicht den Leser umfasst. Die Lesart eines Pluralis Modestiae, bei dem die Referenz sich ausschließlich auf den Autor bezieht, ist hier jedoch ausgeschlossen. Dies wird dadurch erreicht, dass die betroffenen weiteren Personen explizit benannt werden.

(67) Suppose I were a complier and moved further south in Chicago (figs. 2 and 5) to satisfy the voucher requirement. If I or my children had friends or family back in the old neighborhood who were voucher eligible, and we complained about the hardship of moving or, to the contrary, expressed enthusiasm about the new neighborhood, these social interactions could have influenced the moving decisions or destinations of those in our network-especially given the multiyear window of randomization (and thus lease-up) and the fact that many compliers drifted back to poor neighborhoods. (Sampson 2008)

(68) We talk a little about the work system, and both Gun and Kiner say they don't see any reason to rotate. (Vallas 2006)

In Beispiel 67 ist das WIR rückbezogen auf das Subjekt des vorhergehenden Satzes „I or my children“ und insofern ist es ausgeschlossen, den Leser mit in die Bedeutung des WIR zu integrieren. Und auch Beispiel 68 ist eindeutig in seinem Bezug auf die Gruppe, die das WIR bezeichnet, nämlich neben dem Autor die beiden genannten Personen "Gun" und „Kiner" und ggf. noch andere Personen, die in der Situation, auf die der Autor sich bezieht, möglicherweise anwesend waren. Der Leser wird durch diesen direkten situativen Bezug aber definitiv ausgeklammert. Im deutschen Korpus haben sich keine Beispiele für diesen WIR-Gebrauch gefunden.

\subsubsection{Pseudo-inklusives WIR}

Da das inklusive WIR, anders als das ICH, die Wirkung hat, eine direkte Beziehung zwischen dem Leser und dem Autor hervorzurufen (s. Kapitel 3.3.1.2), kann man bei einigen der in dieser Kategorie zusammengefassten Beispiele eine „Pseudo-Inklusion“ interpretieren. Dies ist dann der Fall, wenn die dargestellten Handlungen, die das WIR 
ausführt, den Leser logisch nicht mit einschließen können, es im Text aber so "klingt“, als ob er inkludiert wäre. Dies dient dazu, die Beziehung zum Leser zu stärken, indem er fiktiv in die Datenanalyse und damit die Argumentation für die im Text präsentierten Schlussfolgerungen mit einbezogen wird. So wird die Illusion erzeugt, dass er diese Schlussfolgerungen teilt, auch wenn er ggf. genauso gut zu anderen Schlüssen hätte kommen können. Dies ist ein eindeutiger Fall von Hylands Engagement-Strategie (s. z. B. Hyland (2005) und auch Kap. 3.2.2).

(69) This analysis is based on a permutation test that compares the observed association of participation shifts and networks, on the one hand, with what we would have expected were participation shifts produced without regard for networks, controlling for sequential constraints and dependencies.(Gibson 2005)

(70) Mit einer Irrtumswahrscheinlichkeit zwischen 5 und 10 Prozent können wir jedoch auf eine negative Beziehung zwischen ökonomischen Abstiegen des Partners und der Zweitgeborenenrate schließen: [...]. (Brose 2008)

In Beispiel 69 ist die eine Person, von der man sicher annehmen kann, dass sie die beschriebenen Vorannahmen hatte, der Autor. Durch das WIR bzw. WE wird hier einerseits der (möglicherweise richtige) Eindruck hervorgerufen, dass darüber hinaus noch andere an der Forschung Beteiligte diese Annahmen geteilt haben. Beim Lesen des Textes wiederum suggeriert das WE hier aber auch dem Leser, dass er die gleichen Vorannahmen auch hat bzw. gehabt hätte, hätte er selber diese Forschung durchgeführt. Dadurch werden diese Vorannahmen, auf denen die Auswahl des methodischen Werkzeugs basiert, als allgemeingültig dargestellt und vom nicht allzu kritischen Leser sicherlich auch so wahrgenommen und nicht in Frage gestellt. Dies erleichtert es dem Autor, den Leser von seinen Ergebnissen und Argumentationen zu überzeugen. Vergleichbares gilt auch für die Schlussfolgerung in Beispiel 70.

\subsubsection{Inklusives WIR}

Das inklusive WIR referiert sowohl auf den Autor als auch auf den Leser. Darüber hinaus lassen sich aufgrund weiterer Personengruppen, die einbezogen werden, unterschiedliche Kategorien fassen (vgl. z. B. Kresta 1995; Hutz 1997; Fløttum u. a.|2006). Diese WIR wurden in vorhergehenden Untersuchungen in "Autor und Leser" (auch Teamwork-WIR), "Autor und Fachcommunity“ (auch Fachkreis-WIR) und „Autor und die weitere Gemeinschaft" (auch Gemeinschafts-WIR) eingeteilt, wobei die weitere Gemeinschaft nicht genauer spezifiziert ist und verschiedene (kulturelle, sprachliche etc.) Gruppierungen bis hin zur ganzen Menschheit umfassen kann (vgl. dazu v. a. Kresta (1995), s. auch Kap. 3.3.1.2).

\subsection{Autor und Leser: Teamwork-WIR}

Dieses WIR bezeichnet ausschließlich den Autor und den Leser und wird entsprechend 
überwiegend im Rahmen der Metakommunikation verwendet. Auch hier steht die Beziehung zwischen Autor und Leser im Vordergrund, allerdings ist es logisch nachvollziehbar, dass der Leser die dargestellten Handlungen (mit)vollzieht. Darüber hinaus sind diese Handlungen im Allgemeinen weniger risikoreich als die des „pseudoinklusiven WIR".

(71) As we shall see in more detail below, our interviewees had all at least felt (and sometimes seen) the presence of God and the spirits. (Madsen 2009)

(72) As we have seen, in this particular case at least, neither of these stories can make sense of all of the data. (Vaisey 2009)

(73) Im Folgenden werden wir uns noch der dritten zu prüfenden Hypothese zuwenden. (Best 2008)

(74) Betrachten wir zwei Beispiele, in denen Ego beidesmal das gegen den Willen von Alter gerichtete Ziel hat, dass Alter sterben solle. (Schulz-Schaeffer 2009)

Die WIR in dieser Kategorie führen überwiegend textstrukturierende Handlungen aus, da diese sowohl den Autor als auch den Leser direkt betreffen. Der Autor nimmt den Leser praktisch "bei der Hand“ und geht mit ihm gemeinsam durch den Text, weist ihn auf Besonderheiten hin usw. Diese Referenz wird in Beispiel 71 dadurch deutlich, dass die mit WIR angesprochene Gruppe die weiteren angekündigten Details zu den im Text besprochenen Interviews bzw. Interviewten erhält. Hier könnte man sogar argumentieren, dass sich das WIR eigentlich nur auf den Leser bezieht und insofern eine Ersetzung des DU ist, da der Autor hier nur als „Guide“ oder „Reiseleitung“ fungieren kann (vgl. Tang und John 1999), in dem Sinne, dass ihm die neuen Informationen, die hier angekündigt werden, natürlich schon bekannt sind.

In Beispiel 72 wird ein Rückverweis realisiert, mit dem dem Leser wichtige Punkte des vorhergehenden Textes noch einmal bewusst gemacht werden, indem der Autor auf ein in gewisser Weise gemeinsam "erlebtes" Geschehen verweist. Auch die beiden deutschen Beispiele 73 und 74 dienen der Textstrukturierung, und auch hier macht es nur Sinn, die WIR so zu deuten, dass der Autor und der Leser gemeinsam die (metaphorisch) beschriebene Handlung vollziehen. Die hier verbal dargestellten Handlungen entsprechen auch denjenigen, die Kresta (1995 296) als typisch für diese WIR-Variante identifiziert hat (s. auch Kap. 3.3.1.2, S. 61).

\subsection{Autor und Fachcommunity: Fachkreis-WIR}

In diesen Beispielen wird das WIR als die scientific community gedeutet, innerhalb derer der Aufsatz entstanden ist und entsprechend (voraussichtlich) rezipiert wird. Insofern schließt dieses WIR wieder den Leser mit ein. Auch diese Gemeinschaft ist nur fiktiv und es lässt sich nicht vorhersagen, wo die Grenzen dieser Community verlaufen. Man kann aber wohl davon ausgehen, dass die Mehrheit der Leser der hier untersuchten Texte im Fach Soziologie arbeiten, so dass dies als die „Default-Community“ 
angesehen werden kann, obwohl möglicherweise auch Leser aus anderen Fachkontexten den Text rezipieren oder die Gruppe durch weitere Spezifikationen eingeschränkt wird, z. B. auf diejenigen Wissenschaftler, die sich mit einem bestimmten Themenbereich auseinandersetzen (s. Beispiel 75).

(75) The question holds obvious interest for those who study mechanisms of inequality in contemporary Russia because, as I show, we cannot understand stratification in Russia during the 1990s without taking into account inequality in getting paid. (Gerber 2006)

(76) We know little about the spatial distribution of racially motivated crime, especially compared to other forms of crime. (Lyons 2007)

(77) Like Zeisel, I believe it is essential that we get social experiments right. (Sampson 2008)

(78) Diese zugegebenermaßen unvollständige Zusammenschau aktueller empirischer Befunde über die Erwerbserträge in Frauen- und Männerberufen belegt, dass wir, mit Ausnahme der Einkommensdimension, über wenig empirisch gesichertes Wissen verfügen. (Trappe 2006)

In Beispiel[75 wird die Referentengruppe sogar eindeutig benannt, nämlich "those who study mechanisms of inequality in contemporary Russia", denn das sind diejenigen Personen, die sich mit der "stratification in Russia during the 1990s" auseinandersetzen. Die Wissenschaftler, die hier direkt angesprochen werden, machen also in diesem Beispiel den Fachkreis aus. In Beispiel 76 wird die Gruppe zwar nicht explizit genannt, aber dennoch liegt hier eine ähnliche Situation vor wie in Beispiel 75. Zwar könnte man hier auch weiterreichende Referenzen annehmen, bis hin zur ganzen Nation oder Menschheit, die sicherlich ebenfalls nur wenig über die räumliche Verteilung rassistisch motivierter Straftaten weiß, aber die sinnvollste Deutung ist diejenige, dass sich das WIR hier auch wieder auf diejenigen Wissenschaftler (und ggf. andere betroffene Personengruppen wie Juristen, Polizisten etc.) bezieht, die sich mit dieser Fragestellung auseinandersetzen.

Die beiden anderen Beispiele sind ebenfalls auf diese Art zu interpretieren. In Beispiel 77 bezieht sich das WIR auf alle diejenigen, die soziale (bzw. sozialwissenschaftliche) Experimente durchführen. Und auch das deutsche Beispiel 78 ist so zu deuten, dass das WIR sich auf einen Kreis aus Fachleuten bezieht. Darüber hinaus zeigt sich in den Beispielen 75 und 77 deutlich, dass die hier beschriebenen WIR eindeutig keine Autoren-WIR sein können, da die Autoren abgrenzend zum WIR auf sich selber explizit mit I verweisen.

\subsection{Autor und die weitere Gemeinschaft: Gemeinschafts-WIR}

Bei diesem Gebrauch von WIR bezieht sich die Referenz auf eine noch weiterreichende 
Gruppe als den wissenschaftlichen Fachkreis, der mit dem Artikel direkt angesprochen wird. Dieses WIR ist am wenigsten spezifisch und kann alle möglichen Gruppierungen basierend auf diversen Zugehörigkeitskriterien umfassen.

(79) We may want the dying to be unburdened by financial worries, but the ideal death today can be staggeringly expensive. (Quinn 2008)

(80) Thus, just as a six-year-old is very good at recognizing incorrect grammar (e.g., she knows that "he are hungry" is incorrect) while remaining unable to explain why it is incorrect, we seem better able to recognize our tacit mental contents than to produce them on demand. (Vaisey 2009)

(81) Still, at a time when many state actors are attempting to make self-evident the state's need for means to "embrace" their populations more tightly than ever before, it is worth reflecting on the historically contingent foundations of much of state-structured social life that we now experience as natural. (Loveman 2005)

(82) Diese Trägerschichten und Gruppen genießen noch nicht jene Freiheiten, wie wir sie hier im Westen gewohnt sind. (Schwinn 2006)

Das WIR in Beispiel 79 ist eindeutig ein inklusives, das aber weder in die Kategorie des Teamwork-WIR noch des Fachkreis-WIR fällt, da es eine über die Fachgemeinschaft hinausreichende Gruppe anspricht. Es dient als Subjekt bzw. Agens für eine Handlung bzw. Einstellung, die nicht auf die Mitglieder des Fachkreises beschränkt ist, sondern zumindest die Mitglieder der gleichen kulturellen oder religiösen Gruppe (darauf deutet das Thema Tod und Sterben hin) umfasst, der sich auch die Autorin zugehörig fühlt. Ggf. könnte man dieses WIR auch mit Bezug zur ganzen Menschheit deuten.

Dieser letztgenannte Bezug wird im Beispiel 80 noch deutlicher, da dieses WIR auf eine allgemeine kognitive Eigenschaft der Spezies Mensch hinweist. Beispiel 81 hingegen ist deutlich eine Referenz auf die nationale bzw. staatliche Gemeinschaft, deren soziales Leben von einem Nationalstaat strukturiert wird. Im letzten Beispiel 82 referiert das WIR auf diejenigen Menschen, die in der "westlichen Welt" leben und die im Gegensatz zu den im vorhergehenden (hier nicht aufgeführten) Satz „neu entstandenen Mittelschichten und jüngeren Generationen Ostasiens“ identifiziert werden.

\subsubsection{Rollen in der ersten Person Plural}

Im Folgenden werden die verschiedenen Rollen, die mit dem WIR realisiert werden, vorgestellt. Dabei orientiere ich mich an den Rollen des ICH und ergänze zwei weitere Rollen, die nur mit dem WIR umgesetzt werden. Ob und inwieweit bestimmte WIR-Rollen mit bestimmten Referenztypen des WIR korrelieren, soll an dieser Stelle 
noch nicht berücksichtigt werden. Dies wird im folgenden Kapitel, in dem es um den Vergleich der Ergebnisse geht, thematisiert.

\subsection{Verfasser}

Da von den insgesamt 320 Vorkommen der ersten Person Plural im gesamten Korpus nur 28 mit Handlungen kombiniert werden, die der Verfasserrolle zuzuschreiben sind, werden diese Handlungen hier nicht weiter ausdifferenziert dargestellt, sondern zusammenhängend beschrieben. In der Verfasserrolle wird das WIR genauso wie das ICH häufig im Rahmen von verbalen und graphischen Darstellungen benutzt und wird insbesondere mit Verben der visuellen Wahrnehmung wie see, sehen, betrachten kombiniert (s. Beispiele 83 bis 85). Darüber hinaus erfüllt das WIR hier auch die Funktion des Guide (Tang und John 1999), indem es den Leser durch den Text führt (Beispiele 86 bis 89). Der Autor lenkt, ähnlich wie ein Reiseführer, die Aufmerksamkeit des Lesers auf bestimmte "Sehenswürdigkeiten“, wobei die Auswahl der Verben die Bewegung im Raum simuliert (vgl. v. a. Beispiel 88). Weiterhin vollzieht das WIR auch Handlungen der Textstrukturierung wie zusammenfassen (Beispiel 90).

(83) While he is concerned with the outcome of trust, here we see a number of conversational outcomes: piggybacking is associated with strong ties but not by their constituent simplex ties, while one of the most radical acts of redirection is associated with the simplex ties only. (Gibson 2005)

(84) Die Berufspositionen werden von Bourdieu, wie wir in Kapitel 2 gesehen haben, zur Beschreibung der Klassen und Klassenfraktionen benutzt. (Gerhards 2009)

(85) Betrachten wir zwei Beispiele, in denen Ego beidesmal das gegen den Willen von Alter gerichtete Ziel hat, dass Alter sterben solle. (Schulz-Schaeffer 2009)

(86) While not entirely incompatible, these approaches make different predictions about what we should find to be the most important actors, processes, and outcomes as we turn to the empirics of certification. (Bartley 2007)

(87) Schließlich kommen wir zu den Motiven für die Proteste, die in hohem Maße von konkreten Missständen in den Teilsystemen bestimmt waren (s. Tabelle 1). (Kern 2007)

(88) In between, as we move from left to right, are the classical right-to-rule ideologies (e.g., divine right of kings, traditionalism, prophetic charisma) and what may be called "corrupted" ideologies, resulting in a form of rule that purports legitimacy, but is perceived by most citizens as "cynical." (Straughn 2005)

(89) Damit sind wir bei den Schwächen von Lebensstiltypologien angelangt. (Otte 2005) 
(90) Fassen wir daher noch einmal kurz zusammen, worin die entscheidenden Unterschiede zwischen Bourdieus und Luhmanns Ansatz der Differenzierung liegt: [...]. (Becker 2005)

\subsection{Forscher}

Wie auch dem ICH werden dem WIR Handlungen zugeschrieben, die auf die Identitätsfacette des Forschers hinweisen. Neben denjenigen, die auch schon für das ICH aufgeführt wurden (s. S. 104 ff.), haben sich beim WIR noch zwei weitere ergeben, nämlich der "Appell an die Community" und der "Bezug auf das Community-Wissen”. Dafür wurde in keinem der untersuchten Text die Handlung „Danksagungen“ mit WIR realisiert.

\section{Fokussieren der Forschungsfrage}

Hier geht es ebenso, wie wenn diese Handlung mit dem ICH umgesetzt wird, um den Bezug zur zugrundeliegenden Forschungsfrage und nicht allein um die Frage, die in dem vorliegenden Text behandelt werden soll. Die hier erfassten Handlungen sind daher textübergreifend zu betrachten.

(91) What we want to know, then, is whether there is some association between the relationships people reported having with one another and the P-ties they were observed to produce relative to one another. (Gibson 2005)

(92) The two-and-a-half-year lag between waves may be longer than ideal, but since we are interested in durable moral dispositions, a lag of this length should not pose a significant problem. (Vaisey 2009)

In Beispiel 91 liegt das Erkenntnisinteresse, so wie es hier dargestellt wird, der Forschung im Allgemeinen zugrunde und ist nicht nur ein Teilaspekt, der speziell in diesem Text thematisiert wird. Und auch Beispiel 92 zeigt deutlich, dass das untersuchte Phänomen die Basis des Forschungsvorhabens darstellt und nicht nur im Rahmen des Textes in den Vordergrund gestellt wird.

\section{Darstellen von Hypothesen, Ausgangsannahmen und theoretische Grundpositionen} Auch die Darstellung von Hypothesen, Ausgangsannahmen und theoretischen Grundpositionen wird gelegentlich mit dem WIR realisiert. Hier geht es, wie schon für das ICH beschrieben, darum, die im Normalfall bereits vor dem Verfassen des Textes festgelegten Perspektiven, die der im Text dargestellten Untersuchung zugrunde liegen, zu erläutern.

Interessant ist an den folgenden Beispielen besonders die unterschiedliche Referenz des WIR. Während es sich in Beispiel 93 um ein exklusives Autoren-WIR handelt, das sich vermutlich auf die Arbeitsgruppe um den Autor, wenn nicht sogar nur auf den Autor alleine bezieht, ist das Beispiel 95 eindeutig inklusiv zu werten. Dennoch sind 
natürlich die Leser nicht tatsächlich an der Ausarbeitung der dargestellten Hypothesen beteiligt gewesen. Hier wird die besondere rhetorische und persuasive Stärke des pseudo-inklusiven WIR9 deutlich.

(93) We expected this effect on the grounds that feminized occupations in Russia have greater downward wage flexibility and carry greater risks of negative feedback. (Gerber 2006)

(94) Following the defended communities model, we would expect most hate crime to occur where (1) the incentive to protect identities rooted in racial homogeneity from the threat of racial invasion [...] coexists with (2) the social resources needed to exclude outsiders. (Lyons 2007)

(95) Dieses hier nur kurz eingeführte Handlungsmodell erlaubt die Formulierung eines sozialen Mechanismus für die Auswahl von Handlungsalternativen: Wir können erstens davon ausgehen, dass Akteure zielgerichtet handeln, dass sie also Handlungen auswählen werden, von denen sie annehmen, dass sie ihren Präferenzen oder Vorlieben entsprechen. (Rössel 2006)

\section{Datenerhebung und -analyse}

Wie auch das ICH wird das WIR häufig in Zusammenhang mit Handlungen benutzt, die die Forschungsmethodologie im weitesten Sinne betreffen, also vorrangig mit der Datenerhebung und der Auswertung. Da das WIR jedoch mit Handlungen, die die Datenanalyse beschreiben, nicht so häufig kombiniert wird, werden diese beiden Kategorien hier zusammengefasst. Die Beispiele 96 und 98 stehen für die Datenerhebung, die Beispiele 97 und 99 für die Auswertung. Es wird beim Vergleich der Beispiele auch hier wieder deutlich, dass es häufig unterschiedliche Referenzmöglichkeiten für das WIR gibt. In Beispiel 97 wird z. B. das „pseudo-inklusive WIR“ verwendet, da beim Benennen der Variablen der Leser mit Sicherheit nicht mit eingeschlossen war, es hier aber so dargestellt wird, als sei er involviert gewesen. Dies wird insbesondere durch das can unterstützt, wodurch suggeriert wird, das dieser Prozess noch offen ist und der Leser den folgenden Benennungsvorschlag nicht notwendigerweise akzeptieren muss. So soll der Leser an ausgewählten Stellen den Eindruck der Entscheidungsfreiheit (wenn auch nur in kleinen Dingen) erhalten, damit er auch der weiteren Argumentation des Textes praktisch "gefühlt" freiwillig und bewusst folgt.

(96) Yet the Catholics we interviewed at Transfiguration said that they could not have achieved such a deep religious life if they had stayed in the rigid, rulebound, pre-Vatican II church, and the Jews at Beth Emmanuel said that they could not have achieved it if they had stayed within the relatively loose confines of Reform or Conservative synagogues. (Madsen 2009)

\footnotetext{
${ }^{9}$ Die Verteilung der verschiedenen Rollen nach Referenztypen wird, wie oben erwähnt, nicht hier, sondern im nächsten Kapitel behandelt.
} 
(97) We can denote the individual responsible for the manufacture of a P-shift as i. (Gibson 2005)

(98) Wir haben für die Spielzeiten 2002/2003 und 2003/2004 die Programme der über das Internet zugänglichen Opernhäuser weltweit erhoben. (Gerhardts 2008)

(99) Um zu prüfen, ob dieses Ergebnis durch einen nicht-linearen Effekt der Arbeitslosenquote auf die berufliche Abstiegsmobilität hervorgerufen wird, haben wir in einer weiteren Modellspezifikation die Arbeitslosenquote sowohl in linearer als auch in quadrierter Form berücksichtigt. (Pollmann-Schuldt 2006)

\section{Darstellung der Ergebnisse}

Ähnlich wie dem ICH (s. Kap. 5.1.1.2.5, S. 108) wird auch dem WIR das Erkennen und Erarbeiten von Ergebnissen und Resultaten zugesprochen. Dies kann einerseits dadurch hervorgeheben werden, dass diese Handlung in der Vergangenheit dargestellt wird und damit eindeutig dem direkten Erstellungsprozess des Artikels vorgelagert wird, wie in Beispiel 100. Aber auch bei der Darstellung im Präsens, wie in den anderen Beispielen, liegt die Erkenntnis, die beschrieben wird, außerhalb des Schreibprozesses 10 Hier wird noch einmal die rhetorische Kraft der ersten Person Plural deutlich, durch die es so wirkt, als kämen Autor und Leser während des Leseprozesses gemeinsam zu dem beschriebenen Ergebnis.

(100) Analyzing longitudinal trajectories of Chicago residents (again using PHDCN) no matter where they moved in the United States, we found results that suggest several implications for understanding neighborhood change and, thereby, neighborhood effects. (Sampson 2008)

(101) What we see is that P-shift frequencies and likelihoods varied greatly in these groups, with some very common and others very uncommon. (Gibson 2005)

(102) We see considerable circulation within that poor sector as well as moves further south to other disadvantaged neighborhoods, a pattern similar to the MTO flows. (Sampson 2008)

(103) Mit einer Irrtumswahrscheinlichkeit zwischen 5 und 10 Prozent können wir jedoch auf eine negative Beziehung zwischen ökonomischen Abstiegen des Partners und der Zweitgeborenenrate schließen: [...]. (Brose 2008)

\section{Argumentieren für den eigenen Standpunkt}

Das Argumentieren für den eigenen Standpunkt wird nur in wenigen Fällen mit der ersten Person Plural kombiniert (s. auch Kap. 5.2.2.2). Im ersten Beispiel deutet der weitere Kotext for the sake of the current argument darauf hin, dass hier die Annahme

\footnotetext{
${ }^{10}$ Dies gilt auch dann, wenn der Erkenntnisprozess parallel zum Schreibprozess verläuft, da die Ergebnisse natürlich nicht an den konkreten Text gebunden sind, sondern, wenn sie einmal erzielt wurden, in diversen unterschiedlichen Texten präsentiert werden können.
} 
(assume) nicht als Formulierung von Hypothesen und Ausgangsannahmen der Forschung interpretiert werden kann, sondern als Voraussetzung, von der aus der Autor für seine Ergebnisse argumentiert. Und auch im zweiten Beispiel ist das betreffende see in then we can see so gemeint, dass die Argumentation eingesehen werden soll. Eine so eindeutige Formulierung wie „we argue“, wie sie äquivalent mit der ersten Person Singular sehr häufig vorkommt, wandten die Autoren der untersuchten Texte nicht an.

(104) If we assume for the sake of the current argument that the selection model is reasonable and that the Robins inverse probability of treatment weighting (IPTW) method adjusts for baseline (wave 0) and time-varying confounding, the implications for MTO are significant. (Sampson 2008)

(105) For example, if we understand "identity" as having an unconscious component (our intuitions about "the kind of people we are") and a conscious component (our discursive "identity projects"), then we can see how identities can be thought of-without contradiction-both as motives and as "cultural tools" that we can "pick up and put down" (Swidler 2001, p. 24). (Vaisey 2009)

\section{Begriffe bilden}

Von den für die erste Person Singular beschriebenen Handlungen im Rahmen des Hervorbringens neuen Wissens wird mit dem WIR nur die Begriffsbildung umgesetzt. Und auch hier werden, wie beim ICH, neue Bezeichnungen für relevante Konzepte geprägt und ggf. in den Diskurs eingeführt. Diese Handlungen werden nur in den englischen Texten mit dem WIR kombiniert.

(106) Where citizens become convinced that such goals and principles do not (or no longer) provide a credible check on state action, we may speak of a system of "cynical domination." (Straughn 2005)

(107) The time therefore appears ripe for an interdisciplinary assessment of what we might term the "neighborhood question, experimental style." (Sampson 2008)

\section{Appellieren an die Community}

Eine Handlung, die nicht mit der ersten Person Singular umgesetzt wird, ist das Appellieren an die Community, das als Äquivalent zum Argumentieren gewertet werden kann. Hier stellt das WIR Forderungen an sich selbst, häufig auch modal verstärkt durch should oder sogar must, und damit an die Community oder zumindest die Leser (alle Vorkommen sind eindeutig als inklusiv zu werten). Auf diese Weise kann der Autor ohne (übermäßigen) Gesichtsverlust argumentieren, was seiner Meinung nach wichtige Punkte und Perspektiven sind, die die Community weiter verfolgen sollte. Auch diese Handlung wird nur von den englischsprachigen Autoren durchgeführt. 
(108) To see why inequality in getting paid should operate differently from inequality in contracted earnings, we must consider how contracted earnings are determined. (Gerber 2006)

(109) Lieberson and Lynn (2002) argue that sociology has lost its way in trying to mimic a classical physics-like focus on determinism, whereas instead we should think more like evolutionary biologists. (Sampson 2008)

(110) After all, observational research will continue to be the workhorse of social science, so we might as well get it right, too. (Sampson 2008)

\section{Bezug auf Community-Wissen}

Eine weitere Handlung, die nicht mit der ersten Person Singular auftritt, ist der Bezug auf das Community-Wissen. Hier stellt der Autor seine Annahmen darüber dar, was in der Community an Wissen vorhanden ist (die ersten beiden WE in Beispiel 111) bzw. was nicht vorhanden ist (das letzte WE in Beispiel 111 und Beipiel 114) und welche weiteren Forschungen oder Blickwinkel beispielsweise aufgrund der Datenlage möglich oder unmöglich sind (Beispiele 112 und 113). Anders als beim Appell an die Community ist hier aber nicht die Forderung eingeschlossen, diese auch umzusetzen.

(111) Consequently, while we know much about the historical dynamics that concentrated military, political, and economic power in the modern state (and there are several competing theories that seek to explain the rise of modern states construed in these terms), and we know quite a bit about how states exercise symbolic power in the modern world, we know relatively little about how the modern state accumulated symbolic power. (Loveman 2005)

(112) As more information on the globalization of American industries becomes available, we may be able to determine what role, if any, capital mobility has played in the declining fortunes of organized labor and how unions respond to this challenge. (Martin 2008)

(113) There are no surviving data for the one period of significant rebellion, the Revolution and Civil War of 1640-49, so we cannot determine whether revenue declined during that decade of internal armed opposition to the state, as the fiscal-military model would predict. (Lachmann 2009)

(114) Diese zugegebenermaßen unvollständige Zusammenschau aktueller empirischer Befunde über die Erwerbserträge in Frauen-und Männerberufen belegt, dass wir, mit Ausnahme der Einkommensdimension, über wenig empirisch gesichertes Wissen verfügen. (Trappe 2006) 


\subsubsection{Unpersönliches MAN}

Das unpersönliche MAN hat, ebenso wie das WIR, verschiedene Referenzmöglichkeiten. Diese reichen von der exklusiven Referenz auf den Autor (Beispiele 115 bis 118) bis hin zu den verschiedenen inklusiven Referenzen auf eine unterschiedlich ausgeprägte Gruppe, die sowohl den Autor als auch den Leser einschließt (Beispiele 122 bis 131). Darüber hinaus gibt es aber auch eine Gruppe MANs, die sich weder auf den Autor noch auf den Leser bezieht und tatsächlich auf eine unbestimmte Gruppe anderer Personen referiert (s. Beispiele 119 bis 121).

\subsubsection{Exklusives MAN}

\subsection{Nur Autor}

In den folgenden Beispielen kann sich das MAN nur exklusiv auf den Autor des jeweiligen Textes beziehen, da dieser logisch betrachtet die einzige Person ist, die die dargestellte Tätigkeit ausüben kann bzw. konnte.

(115) That may well be true, but one wonders how such a strong inference could be drawn from a study that randomly assigned housing vouchers to individual families rather than improving neighborhoods. (Sampson 2008)

(116) The research design has the advantage of helping optimize the reliability of the biographical data by permitting one to assess the consistency of responses over several years. (Straughn 2005)

(117) Ganz ohne Bewertung der beiden Erklärungsmodelle kann man wohl feststellen, dass sie alternative methodologische Grundkonzeptionen darstellen. (Albert 2005)

(118) Berechnet man den Korrelationskoeffizienten zwischen dem Mittelwert der Hochkulturorientierung der Länder (Tabelle 1) und dem Anteil der Erwerbspersonen im Kultursektor, dann erhält man einen Wert von 0,787 ( $p<0,001)$. (Gerhards 2009)

In Beispiel 115 ist es klar, dass nur der Autor derjenige sein kann, der sich die Frage stellt, die er hier beschreibt und auch in Beispiel 116 ist der Autor die Person (abgesehen von Mitgliedern einer möglichen Arbeitsgruppe), die Zugang zu den Daten hat, die hier beschrieben werden. Noch deutlicher ist der Autorbezug des MAN in den deutschen Beispielen. In Beispiel 117beispielsweise ist es natürlich der Autor, der im Rahmen des Textes die Handlung des Feststellens vollführt und in Beispiel 118 die besagten Berechnungen durchführt.

\subsection{Nicht Autor}

Neben dieser nur auf den Autor und nicht auf den Leser referierenden Variante gibt es 
jedoch auch die Möglichkeit, dass das MAN sowohl den Leser als auch den Autor des Textes exkludiert bzw. auf eine Gruppe referiert, die Autor und Leser nicht (notwendigerweise) einschließt.

(119) These congregants assumed, first of all, that one voluntarily choose one's connection with the sacred. (Madsen 2009)

(120) Or does being a present-day leader make one more likely to remember one's past mobilization as arising from political consciousness? (Viterna 2006)

(121) CM further argue that the more time one spends in poverty, the greater the neighborhood effect is likely to be, which leads them to conduct an analysis of time spent in poverty, controlling for treatment-group status and background factors. (Sampson 2008)

In Beispiel 119 beschreibt das ONE die Mitglieder der beschriebenen Glaubensgemeinschaft, zu der (vermutlich) weder der Autor des Textes, noch die Leser, auf die dieser Text ausgerichtet ist, zählen. Ähnliches gilt auch für Beispiel 120. Hier schließt das ONE die „present-day leaders" ein, die ebenfalls Autor und Leser nicht mit einschlieBen, während sich in Beispiel 120 das ONE auf diejenigen Personen bezieht, die in Armut leben. Diese Verwendungsweise erinnert an das repräsentative WIR, bei der das WIR sich auf eine sehr große Gruppe wie z. B. eine Nation oder sogar die Menschheit bezieht. Der Unterschied ist aber, dass das repräsentative WIR entsprechend inklusiv funktioniert, während das MAN in der hier beschriebenen Funktion zwar ähnliche Gruppen beschreiben kann, sich aber weder Autor noch Leser diesen Gruppen zuschreiben (müssen). Allerdings besteht durch die Vagheit des MAN natürlich trotzdem die Möglichkeit, diese Zuschreibung zu treffen, wenn sie denn angemessen ist. Ein Leser, der beispielsweise zu der von Madsen 2009 beschriebenen Glaubensgemeinschaft gehört, kann das ONE in Beipiel 119 also entsprechend inklusiv werten.

\subsubsection{Inklusives MAN}

\subsection{Teamwork-MAN}

Dieses MAN bezieht sich, wie auch das entsprechende „Teamwork-WIR“, auf den Autor und den Leser.

(122) Furthermore, one must bear in mind that the Central Americans' legal circumstances are embedded in a larger framework of stiffer immigration law. (Menjivar 2006)

(123) The meaning of religious beliefs and practices is expressed through all three of these forms of symbols, but one should not assume that the three forms are necessarily congruent. (Madsen 2009)

In Beispiel 122 sind es eindeutig der Autor und vor allem der Leser, für die die Aussage des Objektsatzes relevant ist und die sie daher berücksichtigen sollten. Hier könnte 
man ggf. sogar soweit gehen, das MAN nur als auf den Leser bezogen zu interpretieren. Ähnlich verhält es sich mit dem nächsten Beispiel 123 . Auch hier sind die Personen, die Gefahr laufen könnten, die Kongruenz der im vorhergehenden Text benannten religiösen Symbole zu vermuten, diejenigen, die sich mit dem Text und den in ihm bearbeiteten Fragestellungen auseinandersetzen, nämlich der Autor und vor allem der Leser.

\subsection{Fachkreis-MAN}

Das Fachkreis-MAN bezeichnet, äquivalent zum Fachkreis-WIR, die disziplinäre Community und richtet sich also in erster Linie an Soziologen bzw. Wissenschaftler, die sich mit dem im jeweiligen Artikel behandelten Themengebiet auseinandersetzen.

(124) To understand rationalized reconciliation, one must first understand how the secondary market for life insurance changed in the mid-1990s. (Quinn 2008)

(125) Given the distinguished intellectual heritage of the unstated premise that moral judgment must necessarily be a form of conscious deliberation, one can hardly fault repertoire theorists for not questioning it. (Vaisey 2009)

(126) Die Annahme der Existenz stark emergenter Eigenschaften im Bereich des Sozialen hat gewisse Konsequenzen, denen man sich - zumindest auf dem gegenwärtigen Diskussionsstand - nicht entziehen kann [...]. (Albert 2005)

(127) Bourdieu ist in seiner Unterscheidung der Begriffe inkorporiertes kulturelles Kapitel, Habitus, Geschmack und Lebensstil nicht sehr genau, so dass seine Ausführungen unterschiedliche Interpretationen der Begriffe nahe legen, die man entsprechend auch in der Sekundärliteratur findet. (Gerhards 2009)

In Beispiel 124 ist es entsprechend die wissenschaftliche Gemeinschaft, die sich mit den angesprochenen Themen auseinandersetzt und die dann auch die Funktionsweise der sekundären Märkte verstehen muss. Ebenso ist es in Beispiel[125 der Fachkreis, der ggf. Kritik an den „repertoire theorists“ ausüben wird. Ähnlich ist es in den deutschen Bespielen, wo es ebenfalls in erster Linie der Fachkreis ist, den die in Beispiel 126 beschriebenen Konsequenzen betreffen und der in Beispiel 127 die Sekundärliteratur zu Bourdieus Begrifflichkeiten zu Rate zieht.

\subsection{Gemeinschafts-MAN}

Zuletzt hat das MAN natürlich auch die Referenzvariante auf eine größere Gemeinschaft, für die es als Repräsentant steht. Diese kann die Menschheit umfassen (Beispiele 128 und 131) oder auch anders definierte Gruppen wie eine bestimmte Sprachgruppe (eine Interpretationsmöglichkeit für Beispiel 129) oder die Mitglieder einer bestimmten Gesellschaft (Beispiel 130). 
(128) This is consistent with Wolfson's (1988) "theory of the bulge," according to which one engages in a wider range of sociolinguistic behaviors when interacting with, on the one side, familiars, and on the other, complete strangers, whereas most circumspection is exercised when interacting with those falling in between. (Gibson 2005)

(129) His announcement introduced a revolutionary program that dismantled the long-standing categorical divisions between deserving and undeserving poor, but one would have never known it by his rhetoric. (Steensland 2006)

(130) Der in den Kunstpraktiken der Menschen zum Ausdruck kommende Geschmack ist nun keine „harte“ Ressource wie das Einkommen und das Vermögen; der gesellschaftliche Wert der Kunst besteht für seine Nutzer in seinem symbolischen Wert, in der Anerkennung also, die man mit einer bestimmten Kunstpraxis in den Augen der anderen Gesellschaftsmitglieder genießt und mit der man sich gegenüber anderen Mitgliedern der Gesellschaft abgrenzen kann. (Gerhards 2009)

(131) Empfindungen und visceral factors tragen so wesentlich zur Bewertung der Opportunitätskosten bei und bedingen, was überhaupt als Opportunität gelten soll: Ist man sehr hungrig, so ist der Verzicht auf einen Kinobesuch nicht schmerzlich, hat man jedoch gerade gegessen, so ist ein weiteres Sandwich keine lohnende Opportunität, der Verzicht stellt damit auch keine Kosten dar. (Schnabel 2005)

\subsubsection{Rollen mit dem unpersönlichen MAN}

Da das MAN hier nur als Ergänzung zu den Gebrauchsformen der ersten Person betrachtet wird, werden in diesem Kapitel nur diejenigen Vorkommnisse berücksichtigt, die eindeutig einer der Rollen zugeordnet werden können, die auch für die erste Person beschrieben wurden. Ausgeschlossen werden daher insbesondere alle Beispiele, in denen das MAN mit einem Modalverb kombiniert wird, da durch die Modalverben die Rollenzuordnung, die über das Vollverb erfolgt, zu sehr verzerrt wird und in den meisten Fällen das Modalverb die intrinsische Vagheit des unpersönlichen Pronomens noch weiter verstärkt. Aus diesem Grund wird nur beispielhaft vorgestellt, in welchen Rollen das MAN in den untersuchten Texten vergleichbar mit der ersten Person verwendet wird. Auf eine weitere Auswertung über den allgemeinen Überblick im Rahmen des Gesamtvergleichs in Kap. 5.2 hinaus wird im nächsten Kapitel daher verzichtet.

Im Rahmen der Verfasserrolle wird das unpersönliche MAN nur von den deutschen Autoren verwendet und wird vorrangig zur Lesersteuerung, zur Textstrukturierung im Rahmen von Vor- und Rückverweisen sowie zur Begriffsklärung benutzt. Dabei fallen insgesamt 13 Vorkommen in diese Kategorie. In den Beispielen 132 und 
133 fungiert das MAN ähnlich wie das ICH als Guide (Tang und John 1999), indem es die Aufmerksamkeit des Lesers auf die relevanten Inhalte lenkt. Ähnliches ist auch zu Beispiel 134 zu sagen, wo MAN hier als Leser auf einen folgenden Textteil verwiesen wird. In Beispiel 135 schließlich wird eine Definition vorgenommen und der Leser wird darauf hingewiesen, wie er im Rahmen des Artikels den Begriff des „individuellen Lebensstils“ zu verstehen hat.

(132) Betrachtet man nun die unten stehenden Ergebnisse zum Einfluss der Beschäftigung, so fällt auf, dass alle Merkmale einer eingeschränkten oder Nicht-Erwerbstätigkeit die Geburt eines zweiten Kindes extrem positiv bedingen, so dass der Eindruck entsteht, als stünde die Aufnahme einer Vollzeiterwerbstätigkeit nach der Geburt des ersten Kindes ganz klar für eine erwerbs- und vereinbarkeitsorientierte Lebensplanung. (Brose 2008)

(133) Man sieht in Modell 1, dass Landwirte aus Nordrhein-Westfalen (also dem Bundesland, in dem zum Befragungszeitpunkt die höchsten Umstellungsprämien gezahlt wurden) ihren Hof häufiger auf Ökolandbau umstellen als Betriebsleiter in den anderen Bundesländern. (Best 2008)

(134) Die genaue Operationalisierung der erläuterten Variablen findet man im Anhang. (Gerhards 2009)

(135) Dabei versteht man unter dem individuellen Lebensstil „ein relativ stabiles, regelmäßig wiederkehrendes Muster der alltäglichen Lebensführung ... - ein Ensemble von Wertorientierungen, Einstellungen, Deutungen, Geschmackspräferenzen, Handlungen und Interaktionen, die aufeinander bezogen sind" (Geißler 2002: 126). (Isengard 2005)

Neben der Verfasserrolle werden auch die Handlungen der Forscherrolle mit dem unpersönlichen MAN/ONE dargestellt. Es wurden insgesamt 106 Beispiele gefunden (acht englische und 98 deutsche), die in diese Rollenkategorie fallen und die alle den Forscherhandlungen im engeren Sinne zuzuordnen sind. Der Forschungsfokus wird über das MAN bzw. das ONE (ohne Berücksichtigung von Beispielen, deren Handlungen über Modalverben modifiziert werden) nur selten spezifiziert, von den insgesamt fünf Beispielen wurden vier in den englischen Texten gefunden und eines in einem deutschen.

(136) This is particularly the case when one is considering the pervasiveness of corporate litigation since the 1970s (Dunworth and Rogers 1996), which has heightened threats of lengthy legal entanglements, large monetary damages or settlements, and negative publicity. (Skaggs 2008)

(137) Betrachtet man die erwerbsfördernden Makroindikatoren, die Betreuungsangebote verschiedener Altersstufen, fällt auf, dass ein quantitativ gut ausgebautes Betreuungssystem für Drei- bis Fünfjährige nicht unbedingt mit einem 
hohen Ausmaß an Betreuungsplätzen für unter Dreijährige einhergehen muss (Tab. 2). (Berninger 2009)

Etwas häufiger werden dagegen Hypothesen und (theoretische) Grundpositionen, teilweise mit direktem Bezug zur Literatur, dargestellt. Hier gibt es insgesamt 29 Beispiele, die nur aus dem deutschen Korpus stammen. MAN geht von etwas aus (Beispiel 138), MAN unterstellt etwas (Beispiel 139), MAN betrachtet bestimmte theoretische Positionen oder Werke (Beispiel 140) oder schließs sich einer Position an, indem MAN mit Bezug zur Forschungsliteratur etwas voraussetzt (Beispiel 141).

(138) Geht man also nicht von dem recht undeutlichen Begriff einer Steigerung der Wahrscheinlichkeit unwahrscheinlicher Kontakte mittels Liebe aus, der zudem mit keiner Statistik bei Luhmann empirisch belegt wird, sondern von der Reproduktion und damit der Übertragung von Kapitalien, wird deutlich, dass die Wahrscheinlichkeit einer Beziehung der beiden auf die Macht eifersüchtigen Eliten eine klare Veränderung der Kapitalstruktur in der Reproduktion der Macht zur Folge hatte. (Becker 2005)

(139) Dies gilt übrigens auch dann, wenn man für die Partnerwahlen der Individuen ein rationales Handlungskalkül unterstellt, da bei Missachtung der Normen Sanktionen drohen oder andere mit dem Fokus verknüpfte Handlungsziele nicht erreicht werden. (Stauder 2008)

(140) Betrachtet man die Ursprünge der Lebensstildiskussion vor allem in den Arbeiten von Pierre Bourdieu, so richtet sich der theoretische Vorschlag stärker auf die Begriffe Geschmack und Habitus bei Bourdieu und weniger auf seinen Lebensstilbegriff (Bourdieu 1982; Müller 1986). (Rössel 2006)

(141) Auch wenn man mit Boudon (1998: 825) lediglich voraussetzt, dass die individuellen Gründe die Ursache einer rationalen Handlung sein sollen, so mündet das Nichtkennen von Gründen in irrationale Handlungen. (Schnabel 2005)

Auch die Datenerhebung wird vereinzelt mit dem MAN dargestellt und auch hier wurden alle 10 Beispiele im deutschen Korpus gefunden.

(142) Es wird vollständig, wenn man zusätzlich die Kontaktgelegenheiten zu relevanten und verfügbaren Personen erfasst, die durch gemeinsame Bekannte, also durch Transitivität, entstehen können. (Stauder 2008)

(143) Zudem geben Jungen, wenn man sie selber fragt, männliche Lehrkräfte nicht als Vorbilder an (Zimmermann 1999). (Helbig 2010)

(144) Schließlich scheint die Erhebung einer Vielzahl von Variablen erforderlich, weil man getreu dem Motto „Die Masse macht's!“ Lebensstile „ganzheitlich“ zu erfassen hofft. (Otte 2005) 
(145) Man kommt zu begründeteren Einschätzungen dieser Prozesse, wenn man einige Standards kultursoziologischer Analyse berücksichtigt, denen manche Autoren und Studien nicht immer gerecht werden. (Schwinn 2006)

Am häufigsten wurden Handlungen der Datenanalyse mit dem unpersönlichen MAN dargestellt. Hierunter fallen insgesamt 52 der Handlungen, die nicht mit einem Modalverb modifiziert wurden (vier englische, 48 deutsche).

(146) In addition to the naïve comparison, if one fits separate lines for "Republican control" states and "Democratic control" states to a plot of predicted probabilities derived from any of the specifications reported in table 4, it provides visual evidence of a definite (if somewhat nonlinear) interaction between Republican control and electoral competition (results available upon request). (Chen 2007)

(147) A fixed-effects model, the most common type used to analyze longitudinal data with continuous outcome variables, is optimal when one is examining the consequences of events such as discrimination lawsuit filings. (Skaggs 2008)

(148) Multipliziert man Nutzen und Wahrscheinlichkeit einer Konsequenz miteinander, erhält man die Erwartungs-Nutzenkomponente, die sich aus der jeweiligen Konsequenz ergibt (s. Tabelle 1 für eine Zusammenstellung des erwarteten Nutzens der einzelnen Konsequenzen). (Best 2008)

Gemeinsam mit der Analyse werden teilweise auch Ergebnisse genannt, wobei die insgesamt 10 Vorkommnisse wieder nur im deutschen Teilkorpus gefunden wurden.

(149) Vergleicht man zuerst die drei Aggregatskategorien miteinander, dann sieht man, dass ein hochkultureller Lebensstil in den EU-15-Ländern weiter verbreitet ist als in den Ländern der ersten und der zweiten Osterweiterung. (Gerhards 2009)

(150) Betrachtet man den Verlauf nach Geschlechtern getrennt, erkennt man zwei Entwicklungen, die sich vom Gesamtbild zum Teil deutlich unterscheiden. (Helbig 2010)

(151) Man sieht sowohl für die Deutsch- als auch für die Mathematiknote, dass sie maßgeblich durch die Lesekompetenz bzw. Mathematikkompetenz beeinflusst werden. (Helbig 2010)

Darüber hinaus wurde das MAN, ähnlich wie das WIR und das ICH auch in repräsentativer Funktion eingesetzt, was unter der Rolle Andere zusammengefasst wurde. Von den insgesamt 20 Beispielen fallen 16 auf das deutsche und vier auf das englische Korpus.

(152) This is consistent with Wolfson's (1988) "theory of the bulge," according to which one engages in a wider range of sociolinguistic behaviors when interacting with, on the one side, familiars, and on the other, complete strangers, 
whereas most circumspection is exercised when interacting with those falling in between. (Gibson 2005)

(153) $\mathrm{CM}$ further argue that the more time one spends in poverty, the greater the neighborhood effect is likely to be, which leads them to conduct an analysis of time spent in poverty, controlling for treatment-group status and background factors. (Sampson 2008)

(154) Je mehr sich jedoch im Zeitablauf die hohe Frauenerwerbstätigkeit fest etabliert, desto höher wird ganz zwangsläufig die Zahlungsbereitschaft für Dienstleistungen, die man früher vielleicht selbst gemacht hätte. (Nollmann 2006)

(155) Ist einem jemand sympathisch, dann ist man eher bereit zu kooperieren und ist sich seines Gewinns aus dieser Kooperation auch relativ sicher. (Schnabel 2005)

\subsubsection{Zusammenfassung}

In diesem Kapitel wurden die hier relevanten Autorenrollen vorgestellt. Aus der bestehenden Literatur, die in Kap. 3.3.1 diskutiert wurde, haben sich zwei übergeordnete Rollenkategorien ergeben, die Verfasserrolle und die Forscherrolle. Als dritte Kategorie wurden „Andere“ Handlungen zusammengefasst, die nicht in eine der beiden ersten Kategorien fallen. Diesen drei Rollenkategorien wurden weiter differenzierend Handlungskategorien zugeordnet. Diese Handlungskategorien reflektieren reale Handlungen, die im Text durch das Verb in Kombination mit den untersuchten Pronomen als Agens wiedergegeben wird. Aus Effizienzgründen wurden nur diejenigen agentivischen Pronomen berücksichtigt, die gleichzeitig auch das syntaktische Subjekt des jeweiligen Satzes sind.

Für die erste Person Singular wurden als Ausprägungen der Verfasserrolle die Handlungen Verbale und graphische Darstellungen, Darstellen des Ziels bzw. des Fokus des Textes, Theoretischer Hintergrund, Terminologieklärung, Textstrukturierung und Sonstige Metakommunikation unterschieden. Die Forscherrolle zeigt sich im Fokussieren der Forschungsfrage, Darstellen von Hypothesen, Ausgangsannahmen und theoretischen Grundpositionen, Auswählen und Darstellen der Methode und Datenerhebung, Durchführen der Datenaufbereitung und -analyse, Darstellen der Ergebnisse, Argumentieren für den eigenen Standpunkt, Hervorbringen neuer Ideen und Danksagen. Diese Rollen wurden anhand von Beispielen dargestellt und diskutiert.

Für die erste Person Plural wurden zunächst die verschiedenen exklusiven (WIR als Autor, Autor und andere) und inklusiven (Teamwork-, Fachkreis-, GemeinschaftsWIR) Referenzmöglichkeiten aufgezeigt sowie die pseudo-inklusive Referenz erklärt, bei der der Autor eine Handlung, die den Leser logisch nicht mit einschließen kann, trotzdem als WIR-Handlung beschreibt und dadurch der Eindruck entstehen kann, dass der Leser an der Handlung beteiligt ist. Diese Referenzkategorie hat sich in dieser 
Arbeit aufgrund der besonderen Perspektive auf die vom Verb denotierten Handlungen im Gegensatz zu den üblicherweise betrachteten Sprechhandlungen ergeben (s. auch Fløttum u. a. 2006: 98-100). Mit dem Appell an die Community und dem Bezug auf Community-Wissen wurden gegenüber dem ICH zwei neue Kategorien vorgestellt.

Für das unpersönliche MAN wurden ebenfalls zunächst die unterschiedlichen Referenzmöglichkeiten vorgestellt und danach beispielhaft diejenigen Gebrauchsweisen vorgestellt, die mit dem Gebrauch der ersten Person Singular und Plural vergleichbar sind. Im folgenden Abschnitt werden nun die Pronomen im Rahmen ihrer Verwendungsweisen zur Darstellung von Handlungen bzw. Autorenrollen quantitativ und qualitativ verglichen.

\subsection{Die Pronomen im interlingualen Vergleich}

In diesem Abschnitt werden die beiden untersuchten Korpora verglichen, wobei hier zunächst der Gebrauch der drei Pronomen insgesamt dargestellt wird. Im weiteren Verlauf des Abschnitts werden dann die einzelnen in Kap. 5.1.1. 5.1.2 und 5.1.3 vorgestellten Autorenrollen miteinander verglichen. Dabei sind einerseits die Verteilungshäufigkeiten von Belang. Zu diesem Zweck werden beim Vergleich der Korpora bzw. der untersuchten Texte die absoluten Zahlen sowie auf 1000 Wörter normalisierte Zahlen dargestellt. Beim Blick auf die Autorenrollen werden die absoluten Zahlen mit dem prozentualen Anteil, den die jeweilige Anzahl in Bezug auf die Gesamtzahl des betreffenden Pronomens ausmacht, dargestellt.

Weiterhin werden ausgewählte Daten statistisch überprüft, um zu ermitteln, ob die beobachteten Unterschiede zufällig sind oder es einen plausiblen Zusammenhang zwischen den ausgewählten Variablen (Sprache als Hinweis auf die nationale Wissenschaftskultur, Geschlecht, Forschungsansatz und akademischer Status als Hinweis auf die Schreiberfahrung der Autoren) und dem Gebrauch der untersuchten Pronomen gibt. An dieser Stelle sei allerdings noch einmal deutlich auf die begrenzte Aussagekraft insbesondere der Ergebnisse dieser statistischen Auswertungen und den insgesamt rein explorativen Charakter dieser Studie hingewiesen.

Aufgrund der Beschaffenheit des Korpus sowie der untersuchten Kategorien wurde für diese Untersuchung der Log-Likelihood-Test gewählt, den Rayson und Garside (2000 2) für besonders geeignet halten, die Frequenzverteilung von einzelnen Wörtern zu überprüfen. Da sich die Verteilung der LL-Quotienten einer $\chi^{2}$-Verteilung annähert, können die gleichen Signifikanzniveaus wie beim $\chi^{2}$-Test angewandt werden. Weiterhin berücksichtigt der Test die Größe des jeweiligen Korpus, so dass hier mit den absoluten und nicht den normalisierten Ergebnissen gearbeitet werden kann und die unterschiedliche Größe der untersuchten Korpora bzw. Teile daraus keinen verfälschenden Einfluss auf das Ergebnis haben (Rayson und Garside|2000: 3). 


\begin{tabular}{|l|l|l|l|l|}
\hline Korpus & $\begin{array}{l}\text { Anzahl 1. Sg. } \\
\text { (auf 1000 Wör- } \\
\text { ter) }\end{array}$ & $\begin{array}{l}\text { Anzahl 1. Pl. } \\
\text { (auf 1000 Wör- } \\
\text { ter) }\end{array}$ & $\begin{array}{l}\text { Anzahl } \\
\text { MAN/ONE } \\
\text { (auf 1000 } \\
\text { Wörter) }\end{array}$ & $\begin{array}{l}\text { gesamt } \\
\text { (auf 1000 Wör- } \\
\text { ter) }\end{array}$ \\
\hline Englisch & $1311(3,47)$ & $285(0,75)$ & $119(0,32)$ & $1715(4,54)$ \\
\hline Deutsch & $105(0,56)$ & $35(0,19)$ & $300(1,59)$ & $440(2,34)$ \\
\hline
\end{tabular}

Tabelle 5.1: Übersicht alle Pronomen

\subsubsection{Vergleich aller Pronomen}

Zunächst gehe ich hier auf den generellen Gebrauch der drei untersuchten Pronomen ein und nehme dazu die beiden Korpora insgesamt in den Blick. Dabei ist auf den ersten Blick ersichtlich, dass im englischen Korpus deutlich mehr Pronomen verwendet werden und dass insbesondere die erste Person Singular wesentlich häufiger auftritt als im deutschen. Tabelle 5.1 stellt die jeweiligen Zahlen gerechnet auf die Gesamtwortzahl des deutschen bzw. US-amerikanischen Korpus einander gegenüber.

Die beiden Tabellen 5.3 und 5.4 zeigen die Verteilung der Pronomen pro Text. Dabei fällt auf, dass die Pronomen nicht gleichmäßig verteilt sind, dass sie also nicht von allen Autoren gleichmäßig häufig benutzt werden, sondern dass es deutliche individuelle Unterschiede gibt. Während einige Autoren sehr häufig auf die persönliche Darstellungsweise mit den Pronomen der ersten Person zurückgreifen, verwenden andere diese in ihren Texten gar nicht (s. Tab. 5.2). Dies entspricht den Beobachtungen in anderen Untersuchungen (s. z. B. Steinhoff 2007; 171).

Im deutschen Korpus wird in zehn Texten kein ICH benutzt 11 , das WIR kommt ebenfalls in zehn Texten nicht vor und auf das MAN verzichten zwei deutsche Autoren. Demgegenüber verwenden neun amerikanische Autoren kein ONE und fünf kein WE. Die erste Person Singular kommt dagegen in allen amerikanischen Texten vor. Dieses allgemeine Ergebnis ist nicht überraschend, eine vergleichbare Verteilung, zumindest aber die deutliche Präferenz englischsprachiger Autoren, die erste Person Singular in wissenschaftlichen Artikeln zu benutzen, wurde auch in anderen Untersuchungen mehrfach nachgewiesen (s. dazu den Überblick in Kap. 3.3.1). Die Tabellen 5.3 und 5.4 zeigen die Verteilung der Pronomen pro Text, die Abbildungen 5.1 bis 5.6 stellen die Verteilung der einzelnen Pronomen als Säulendiagramme mit aufsteigender Häufigkeit dar.

\footnotetext{
${ }^{11}$ Dieser Wert entspricht dem von Steinhoff, in dessen Korpus in "gut $40 \%$ der Texte [...] das ,ich' nicht zum Einsatz" kommt (Steinhoff|2007 171).
} 


\begin{tabular}{|l|l|l|l|}
\hline Korpus & $\begin{array}{l}\text { Texte ohne 1. Sg. } \\
\text { (in \%) }\end{array}$ & $\begin{array}{l}\text { Texte ohne 1. Pl. } \\
\text { (in \%) }\end{array}$ & $\begin{array}{l}\text { Texte } \\
\text { MAN/ONE } \\
\text { (in \%) }\end{array}$ \\
\hline Englisch & $0(0 \%)$ & $5(20 \%)$ & $9(36 \%)$ \\
\hline Deutsch & $10(40 \%)$ & $10(40 \%)$ & $2(8 \%)$ \\
\hline
\end{tabular}

Tabelle 5.2: Texte ohne Pronomen

\begin{tabular}{|c|c|c|c|}
\hline Name & $\begin{array}{l}\text { Anzahl 1. Sg. ab- } \\
\text { solut } \\
\text { (auf } 1000 \text { Wörter) }\end{array}$ & $\begin{array}{l}\text { Anzahl 1. Pl. abso- } \\
\text { lut } \\
\text { (auf } 1000 \text { Wörter) }\end{array}$ & $\begin{array}{l}\text { Anzahl } \\
\text { MAN/ONE abso- } \\
\text { lut } \\
\text { (auf } 1000 \text { Wörter) }\end{array}$ \\
\hline Bartley & $29(1,72)$ & $4(0,24)$ & $10(0,59)$ \\
\hline Chen & $80(4,01)$ & $0(0)$ & $2(0,10)$ \\
\hline Gerber & $59(3,8)$ & $28(1,81)$ & $1(0,06)$ \\
\hline Gibson & $61(4,82)$ & $32(2,53)$ & $11(0,87)$ \\
\hline Grodsky & $82(5,65)$ & $6(0,41)$ & $6(0,41)$ \\
\hline Kay & $60(3,93)$ & $1(0,07)$ & $0(0)$ \\
\hline King & $46(3,14)$ & $1(0,07)$ & $0(0)$ \\
\hline Lachmann & $8(0,7)$ & $4(0,35)$ & $0(0)$ \\
\hline Loveman & $2(0,08)$ & $10(0,41)$ & $0(0)$ \\
\hline Lyons & $28(2,44)$ & $5(0,43)$ & $1(0,09)$ \\
\hline Madsen & $30(1,72)$ & $35(2,02)$ & $12(0,69)$ \\
\hline Martin & $25(2,54)$ & $3(0,30)$ & $1(0,10)$ \\
\hline Menjivar & $51(3,35)$ & $2(0,13)$ & $3(0,20)$ \\
\hline Pedriana & $34(2,16)$ & $0(0)$ & $3(0,19)$ \\
\hline Quinn & $61(3,71)$ & $5(0,30)$ & $9(0,55)$ \\
\hline Sampson & $71(5,23)$ & $43(3,17)$ & $14(1,03)$ \\
\hline Schrank & $35(3,43)$ & $6(0,59)$ & $0(0)$ \\
\hline Schwartz & $47(4,56)$ & $1(0,10)$ & $0(0)$ \\
\hline Skaggs & $58(5,05)$ & $0(0)$ & $5(0,44)$ \\
\hline Steensland & $6(0,29)$ & $0(0)$ & $0(0)$ \\
\hline Straughn & $40(1,86)$ & $23(1,07)$ & $30(1,39)$ \\
\hline Vaisey & $72(5,44)$ & $36(2,72)$ & $8(0,60)$ \\
\hline Vallas & $32(2,11)$ & $6(0,40)$ & $0(0)$ \\
\hline Vaughan & $220(12,61)$ & $34(1,95)$ & $0(0)$ \\
\hline Viterna & $74(5,72)$ & $0(0)$ & $3(0,22)$ \\
\hline gesamt & $1311(3,47)$ & $285(0,75)$ & $119(0,32)$ \\
\hline
\end{tabular}

Tabelle 5.3: Übersicht alle Pronomen Englisch 


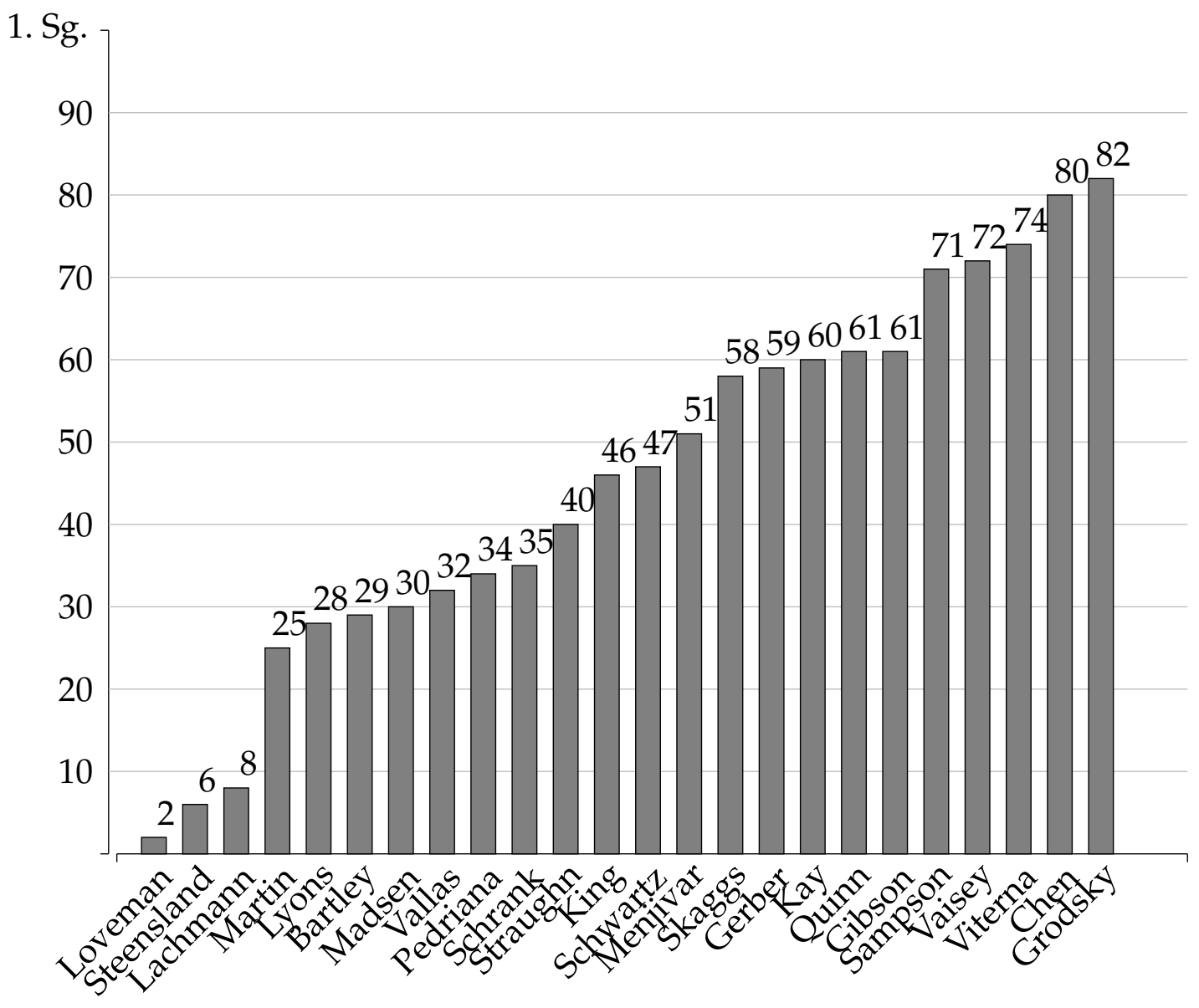

Abbildung 5.1: Übersicht 1. Sg. englisch (ohne Vaughan 2006)

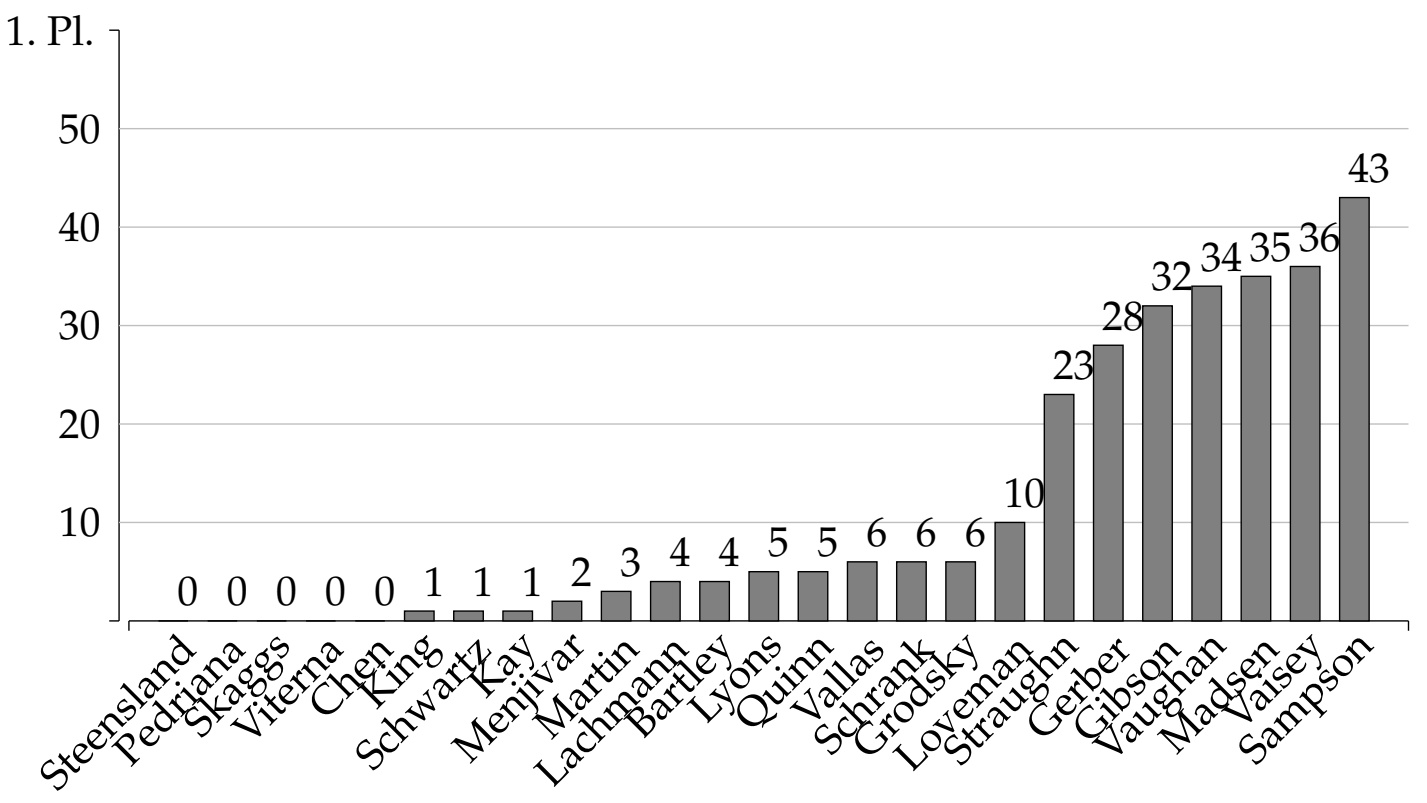

Abbildung 5.2: Übersicht 1. Pl. englisch 


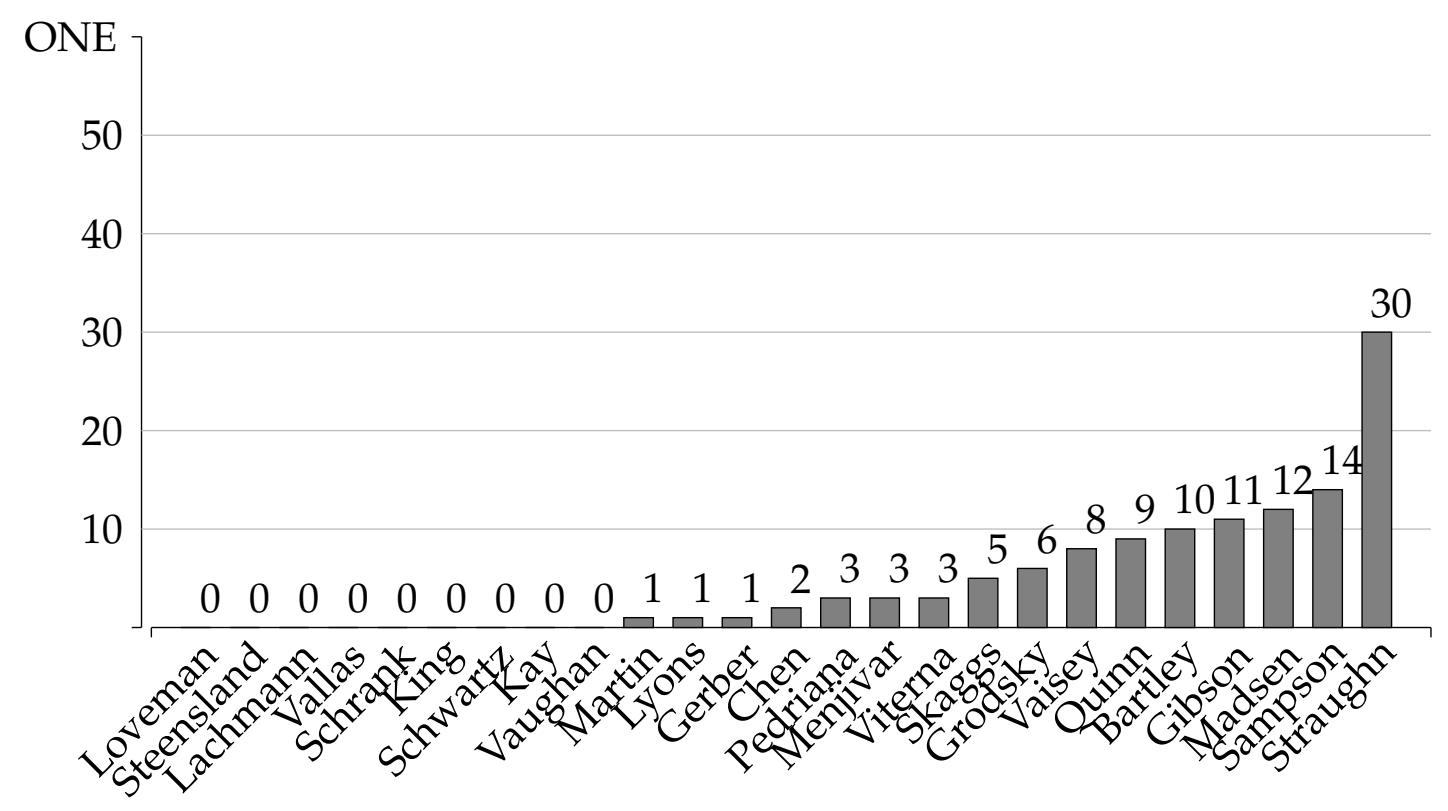

Abbildung 5.3: Übersicht unpers. ONE 


\begin{tabular}{|l|l|l|l|}
\hline Name & $\begin{array}{l}\text { Anzahl 1. Sg. } \\
\text { (auf 1000 Wörter) }\end{array}$ & $\begin{array}{l}\text { Anzahl 1. Pl. } \\
\text { (auf 1000 Wörter) }\end{array}$ & $\begin{array}{l}\text { Anzahl } \\
\text { MAN/ONE } \\
\text { (auf 1000 Wörter) }\end{array}$ \\
\hline Albert & $15(4,41)$ & $4(1,17)$ & $11(3,23)$ \\
\hline Becker & $0(0)$ & $8(0,95)$ & $15(1,78)$ \\
\hline Berninger & $0(0)$ & $0(0)$ & $2(0,25)$ \\
\hline Best & $0(0)$ & $1(0,13)$ & $14(1,86)$ \\
\hline Bienfait & $4(0,51)$ & $1(0,13)$ & $10(1,27)$ \\
\hline Brose & $2(0,35)$ & $2(0,35)$ & $7(1,24)$ \\
\hline Gerhards & $33(4,08)$ & $6(0,74)$ & $37(4,57)$ \\
\hline Helbig & $0(0)$ & $0(0)$ & $29(5,35)$ \\
\hline Isengard & $1(0,14)$ & $0(0)$ & $3(0,43)$ \\
\hline Kern & $2(0,19)$ & $1(0,09)$ & $2(0,19)$ \\
\hline Lohmann & $0(0)$ & $0(0)$ & $19(2,26)$ \\
\hline Lois & $1(0,13)$ & $0(0)$ & $0(0)$ \\
\hline Nollmann & $0(0)$ & $0(0)$ & $1(0,15)$ \\
\hline Nonnenmacher & $0(0)$ & $0(0)$ & $5(0,75)$ \\
\hline Otte & $10(1,01)$ & $1(0,10)$ & $36(3,65)$ \\
\hline Pollmann-Schuldt & $0(0)$ & $3(0,61)$ & $1(0,20)$ \\
\hline Rössel & $2(0,39)$ & $1(0,20)$ & $18(3,52)$ \\
\hline Schnabel & $5(0,44)$ & $1(0,09)$ & $13(1,13)$ \\
\hline Schulz-Schaeffer & $6(0,63)$ & $1(0,11)$ & $25(2,65)$ \\
\hline Schwinn & $13(1,15)$ & $3(0,26)$ & $34(3,00)$ \\
\hline Stauder & $1(0,16)$ & $0(0)$ & $8(1,25)$ \\
\hline Strauß & $3(0,54)$ & $1(0,18)$ & $1(0,18)$ \\
\hline Stein & $0(0)$ & $0(0)$ & $3(0,35)$ \\
\hline Teipen & $7(1,13)$ & $0(0)$ & $6(0,97)$ \\
\hline Trappe & $0(0)$ & $1(0,13)$ & $0(0)$ \\
\hline gesamt & $\mathbf{1 0 5}(\mathbf{0}, \mathbf{5 6})$ & $\mathbf{3 5 ( 0 , 1 9 )}$ & $\mathbf{3 0 0}(\mathbf{1}, \mathbf{5 9 )}$ \\
\hline & & & \\
\hline
\end{tabular}

Tabelle 5.4: Übersicht alle Pronomen Deutsch 
1. Sg. dt.

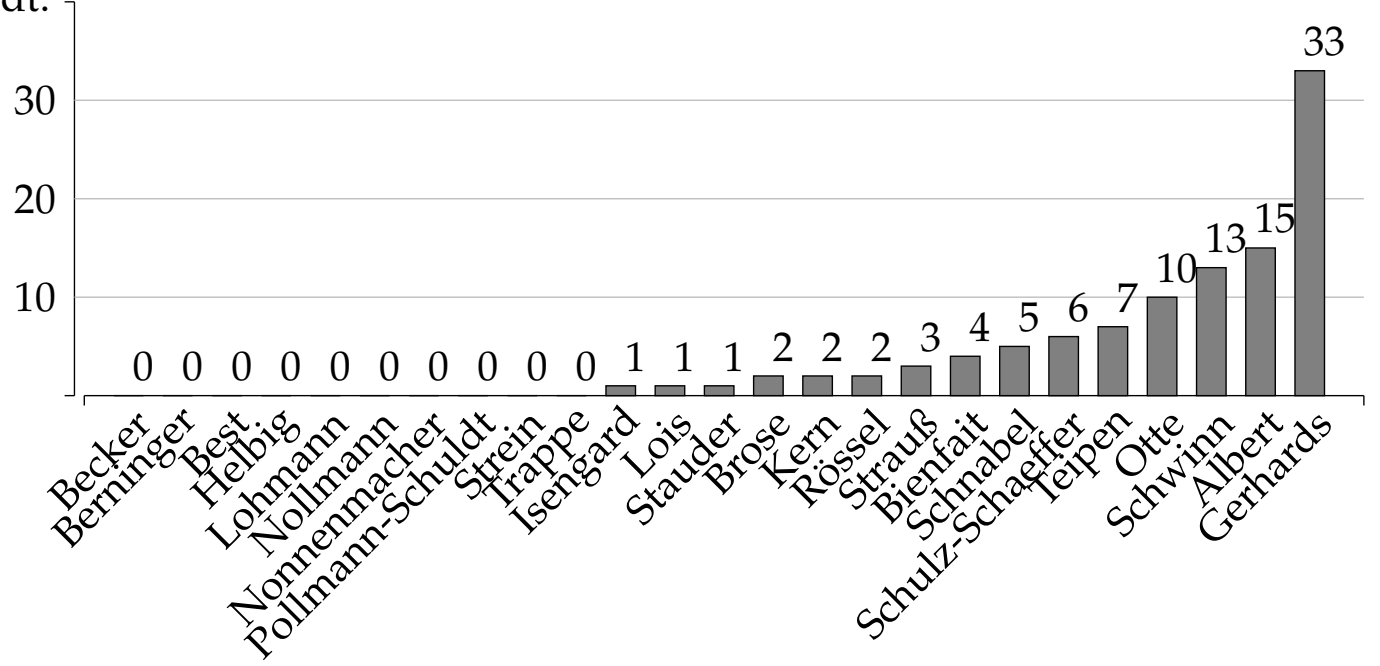

Abbildung 5.4: Übersicht 1. Sg. deutsch

1. Pl. dt.

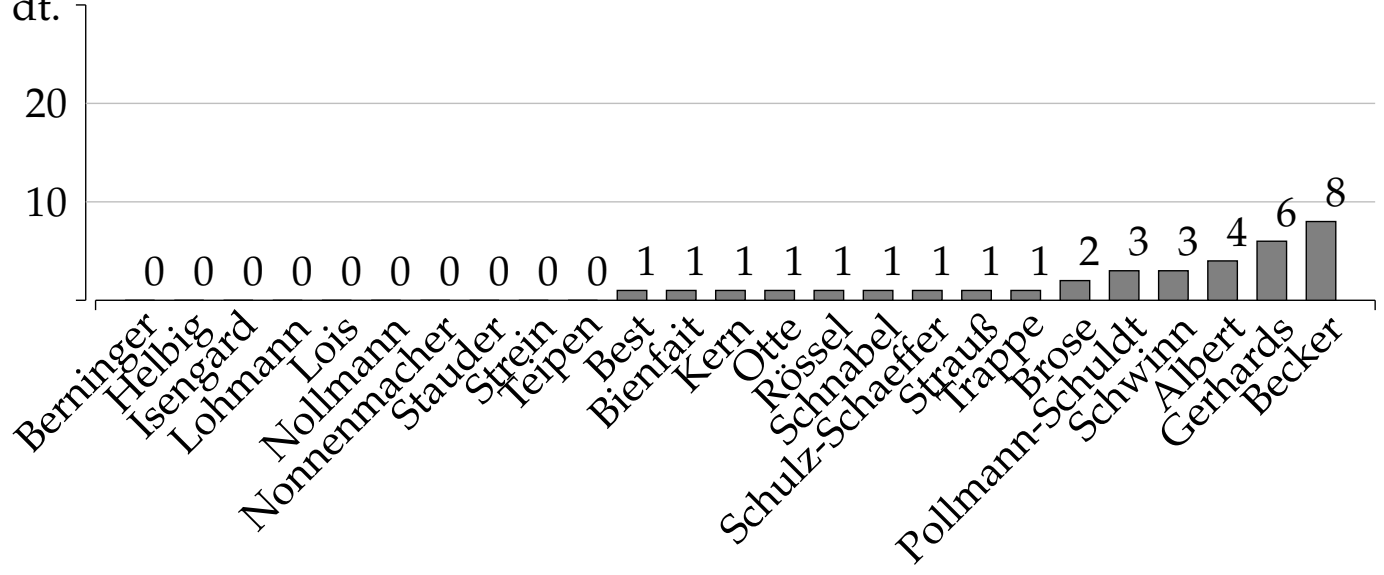

Abbildung 5.5: Übersicht 1. Pl. deutsch

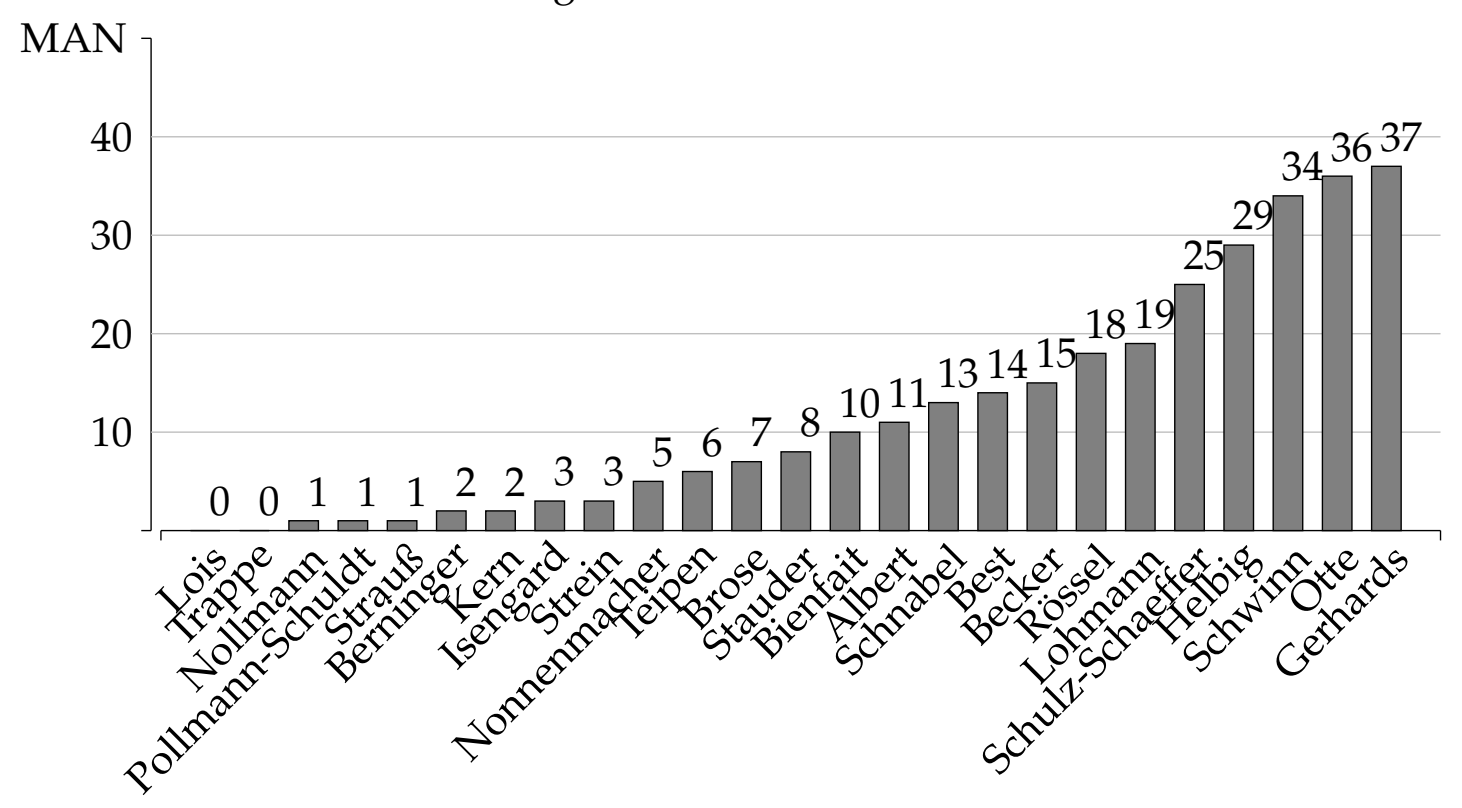

Abbildung 5.6: Übersicht unpersönliches MAN 
Im Folgenden werden die ermittelten Werte für den Gebrauch der drei Pronomen in den beiden Korpora anhand der gängigsten Kennzahlen vorgestellt. Dazu werden zwei Mittelwerte (das arithmetische Mittel und der Median) sowie zwei StreuungsmaBe (die einfache Streuung und die Standardabweichung) herangezogen.

Das arithmetische Mittel $(\bar{x})$ gibt den Durchschnittswert der gemessenen Werte an, also die Summe aller Werte geteilt durch ihre Anzahl (Benninghaus 2007). Das heißt also, dass die mittlere Menge an ICH pro Text im deutschen Korpus bei 0,61 liegt, während im englischen Korpus durchschnittlich 3,22 Mal I (pro 1000 Wörter), also mehr als das Fünffache, auf einen Text kommen 12 .

Der Median ( $\tilde{x}$ gibt an, welcher Wert der nach Größe sortierten Messwerte die Reihe halbiert (Benninghaus 2007; 39), also der Wert, oberhalb und unterhalb dessen es genauso viele kleinere wie größere Werte in der Reihe gibt. Da für das deutsche Korpus alle 25 Texte in die Berechnung mit einbezogen wurden, bedeutet die Zahl 0,16 als Median für die ICH-Vorkommen, dass es 12 Texte gibt, in denen weniger ICHs benutzt wurden und 12, in denen mehr ICHs als 0,16 pro 1000 Wörter vorkamen. An diesem sehr kleinen Wert wird auch noch einmal deutlich, dass eine nennenswerte Menge an deutschen Autoren (10, d. h. 40 \% des gesamten deutschen Korpus) das ICH gar nicht benutzt haben. Für die Betrachtung des ICH-Gebrauchs in den englischen Texten wurde der Text von Diane Vaughan (Vaughan 2006) ausgeklammert, da diese Autorin die erste Person Singular auf sehr untypische Weise gebraucht (s. Kap. 5.3). Daher liegen hier der Berechnung des Medians nur 24 Texte zugrunde.

Die Streuung (Range, Spannweite, $R$ ) ist die Differenz aus dem größten und dem kleinsten Wert und gibt damit an, in welchem Bereich sich die Werte bewegen (Benninghaus 2007; 52). Die Standardabweichung (s) letztlich sagt etwas über die Abweichung aller Messwerte vom arithmetischen Mittel aus (Benninghaus 2007; 58). Diese Werte werden in den Tabellen 5.5 bis 5.7 vorgestellt und in den Tabellen 5.8 und 5.9 noch einmal für die beiden Korpora zusammengefasst. Da, wie in Tabelle 5.3 ersichtlich wird, der Text von Vaughan 2006 weit überdurchschnittlich viele Vorkommnisse der ersten Person Singular enthält, wird er auch in der folgenden Analyse des ICH/IGebrauchs nicht mit berücksichtigt. Statt dessen wird er im Anschluss an dieses Teilkapitel einer Einzelfallanalyse unterzogen (s. Kap. 5.3). In den Tabellen geben die Zahlen in Klammern die Werte an, die sich ergäben, wenn der Text Vaughan 2006 mit einbezogen würde.

\footnotetext{
${ }^{12} \mathrm{Zu}$ den Werten für die erste Person Plural und ONE/MAN s. Tabellen 5.555 .7
} 


\begin{tabular}{|l|l|l|}
\hline & 1. Sg. Englisch & 1. Sg. Deutsch \\
\hline $\bar{x}$ & $3,22(3,50)$ & 0,61 \\
\hline$\tilde{x}$ & $3,42(3,43)$ & 0,16 \\
\hline $\boldsymbol{R}$ & $5,57(12,53)$ & 4,41 \\
\hline $\boldsymbol{s}$ & $1,66(2,48)$ & 1,15 \\
\hline
\end{tabular}

Tabelle 5.5: Vergleich 1. SG

\begin{tabular}{|l|l|l|}
\hline & 1. Pl. Englisch & 1. Pl. Deutsch \\
\hline $\bar{x}$ & 0,76 & 0,21 \\
\hline$\tilde{x}$ & 0,35 & 0,10 \\
\hline $\boldsymbol{R}$ & 3,17 & 1,17 \\
\hline $\boldsymbol{s}$ & 0,98 & 0,32 \\
\hline
\end{tabular}

Tabelle 5.6: Vergleich 1. PL

\begin{tabular}{|l|l|l|}
\hline & ONE & MAN \\
\hline $\bar{x}$ & 0,30 & 1,61 \\
\hline$\tilde{x}$ & 0,10 & 1,24 \\
\hline $\boldsymbol{R}$ & 1,39 & 5,35 \\
\hline $\boldsymbol{s}$ & 0,38 & 1,54 \\
\hline
\end{tabular}

Tabelle 5.7: Vergleich MAN/ONE

\begin{tabular}{|l|l|l|l|}
\hline & 1. Sg. & 1. Pl. & ONE \\
\hline $\bar{x}$ & $3,22(3,50)$ & 0,76 & 0,30 \\
\hline$\tilde{x}$ & $3,42(3,43)$ & 0,35 & 0,10 \\
\hline $\boldsymbol{R}$ & $5,57(12,53)$ & 3,17 & 1,39 \\
\hline $\boldsymbol{s}$ & $1,66(2,48)$ & 0,98 & 0,38 \\
\hline
\end{tabular}

Tabelle 5.8: deskriptive Kennzahlen Englisch

\begin{tabular}{|l|l|l|l|}
\hline & 1. Sg. & 1. Pl. & MAN \\
\hline $\bar{x}$ & 0,61 & 0,21 & 1,61 \\
\hline$\tilde{x}$ & 0,16 & 0,10 & 1,24 \\
\hline $\boldsymbol{R}$ & 4,41 & 1,17 & 5,35 \\
\hline $\boldsymbol{s}$ & 1,15 & 0,32 & 1,54 \\
\hline
\end{tabular}

Tabelle 5.9: deskriptive Kennzahlen Deutsch 
Weiterhin wird nun überprüft, inwiefern neben der Sprache die Parameter Gender, Forschungsansatz und Schreiberfahrung bzw. akademischer Status eine Rolle beim Gebrauch der Pronomen spielen. Dazu wird der Log-Likelihood-Test mit den entsprechenden Teilkorpora berechnet. Zur Berechnung des Tests wird hier der Online-Calculator für den Log-Likelihood-Test von Paul Rayson (University of Lancaster) benutzt, der auch Angaben über die Ausrichtung der Verteilung liefert. ${ }^{13}$ Da der LogLikelihood-Test der gleichen Verteilung folgt, wie der $\chi^{2}$-Test, können hier auch die gleichen Signifikanzniveaus angewandt werden. Die entsprechenden kritischen Werte liegen bei 3,84 für $\mathrm{p}<0,05^{14}$; 6,63 für $\mathrm{p}<0,01 ; 10,83$ für $\mathrm{p}<0,001$ und 15,13 für $\mathrm{p}<$ 0,0001 . Auch an dieser Stelle sei noch einmal darauf hingewiesen, dass hier nur explorative Ziele verfolgt werden und die Aussagekraft der dargestellten Ergebnisse nicht überbewertet werden sollte.

Zunächst werden die beiden Korpora insgesamt miteinander verglichen, die Ergebnisse der Berechnung $\left(G^{2}\right)$ werden in Tabelle 5.10 zusammengefasst. Wie man sieht, sind die $\mathrm{G}^{2}$-Werte für alle drei Vergleiche sehr hoch (und damit die p-Werte sehr niedrig), es kann daher davon ausgegangen werden, dass die Verteilung der Pronomen in den beiden Korpora nicht von der Korpusgröße abhängt, sondern dass die Variable Sprache einen Einfluss auf die beobachteten Häufigkeiten hat. Man kann also tatsächlich davon ausgehen, dass die hier untersuchten US-amerikanischen Autoren häufiger die Pronomen der ersten Person Singular und Plural benutzen als die deutschen und dass die deutschen Autoren häufiger auf das unpersönliche Pronomen zurückgreifen, als die amerikanischen.

\begin{tabular}{|l|l|l|l|}
\hline & 1.Sg. & 1. P1. & MAN/ONE \\
\hline$G^{2}$ & $430,63(543,32)$ & 86,66 & 256,58 \\
\hline
\end{tabular}

Tabelle 5.10: Vergleich $\mathrm{G}^{2}$ im Gesamtkorpus

Ein weiterer möglicher Einflussfaktor ist das Geschlecht der Autoren. Die Frage ist also, ob es einen Unterschied gibt, wie häufig Männer die Pronomen im Vergleich zu Frauen benutzen. Dies wird jeweils innerhalb der beiden Sprachen überprüft, die Frage ist also, ob amerikanische männliche Autoren die Pronomen anders benutzen als amerikanische weibliche Autorinnen und äquivalent dazu, wie der Gebrauch bei den deutschen Autorinnen und Autoren ist. Dazu werden entsprechend die Wortzahlen und

\footnotetext{
${ }^{13}$ http://ucrel.lancs.ac.uk/llwizard.html, (Zugriff: 16.06.2013). Die Ausrichtung der Werte wird der besseren Ubersicht halber in den Tabellen nicht aufgeführt, sondern nur im Text besprochen, wenn sie relevant ist.

${ }^{14}$ Der p-Wert gibt an, wie wahrscheinlich es ist, dass sich die gemessene Frequenzverteilung der Pronomina einfach aus den unterschiedlichen Korpusgrößen ergibt. Je kleiner der p-Wert, desto unwahrscheinlicher ist dies. Üblicherweise werden p-Werte kleiner als 0.05 , kleiner als 0.01 und kleiner als 0.001 unterschieden. Kleinere p-Werte stützen demnach die Annahme, dass es einen tatsächlichen Unterschied zwischen englischen und deutschen Autoren bezüglich der Verwendungshäufigkeit der Pronomen gibt. (Dr. Roland Schäfer, 16.08.2015, persönliche Kommunikation)
} 
die beobachteten Häufigkeiten der jeweiligen Teilkorpora zugrunde gelegt (s. Tabelle 5.11). Auch hier wird für die Analyse der ersten Person Singular der Text von Diane Vaughan nicht berücksichtigt, da ihr Gebrauch der ersten Person Singular deutlich von dem der anderen Autoren abweicht.

\begin{tabular}{|l|l|l|l|l|l|}
\hline & & Wörter & $\mathbf{1 . ~ S g . ~}$ & $\mathbf{1 . P 1 .}$ & MAN/ONE \\
\hline \multirow{4}{*}{ E } & männl. & 253308 & 738 & 232 & 99 \\
\cline { 2 - 6 } & weibl. & 106852 & 353 & 53 & 20 \\
\cline { 2 - 6 } & $G^{\mathbf{2}}$ & - & $\mathbf{3 , 7 1}$ & $\mathbf{2 9 , 2 5}$ & $\mathbf{1 5 , 7 3}$ \\
\hline \multirow{4}{*}{$\mathrm{D}$} & männl. & 113552 & 83 & 29 & 250 \\
\cline { 2 - 7 } & weibl. & 74695 & 22 & 6 & 50 \\
\cline { 2 - 6 } & $G^{\mathbf{2}}$ & - & $\mathbf{1 6 , 7 8}$ & $\mathbf{8 , 3 4}$ & 74,84 \\
\hline
\end{tabular}

Tabelle 5.11: Vergleich nach Geschlecht

Offenbar scheint auch der Faktor Geschlecht in dem hier untersuchten Korpus einen Einfluss auf den Gebrauch der Pronomen auszuüben. Abgesehen von dem Unterschied im ICH-Gebrauch der amerikanischen Autorinnen und Autoren, der knapp unter $\mathrm{p}<0,05(3,84)$ liegt, haben sich für alle anderen Vergleiche Werte ergeben, die die Annahme stützen, dass das Geschlecht der Autoren einen Einfluss hat. Außerdem ist es für diese Fälle durchgängig so, dass die männlichen Autoren die jeweiligen Pronomen häufiger verwenden als die Autorinnen. Nur beim Gebrauch der ersten Person Singular im englischen Korpus besteht die Tendenz, dass die weiblichen Autorinnen das ICH öfter benutzen als ihre männlichen Kollegen, was noch verstärkt würde, wenn der Text von Diane Vaughan mit einbezogen würde.

Eine weitere Hypothese, die sich im Verlauf der Analyse ergeben hat, war, dass der Forschungsansatz der im Text dargestellten Untersuchung einen Einfluss auf die Art hat, wie sich ein Autor in seinem Text darstellt. Die Überlegung, die dieser Annahme zugrunde liegt, ist, dass bei qualitativen Methoden, insbesondere bei ethnographischen Ansätzen, also z. B. Interviews oder Methoden der Feldforschung, der Forscher häufig deutlich stärker und persönlicher in die Datenerhebung involviert ist, als es z. B. bei rein quantitativen Methoden der Fall ist (vgl. z. B. Sanderson|2008a: 140). Insofern könnte man annehmen, dass in den qualitativen Forschungsartikeln besonders, aber nicht nur, bei der Darstellung der Datenerhebung die Person des Forschenden stärker in den Vordergrund tritt, da er im Rahmen qualitativer Verfahren ggf. stärker als Person in Interaktionen mit anderen Personen (z. B. beim Durchführen von Interviews oder der Auswahl von Probanden, s. Beispiel 156) eingebunden ist, als bei der Datenerhebung im Rahmen quantitativer Methoden.

(156) In fact, I had more difficulty explaining to nonselected individuals why I was not going to interview them than I had convincing selected respondents to participate. (Viterna 2006) 


\begin{tabular}{|l|l|l|l|l|l|}
\hline & & Wörter & $\mathbf{1 . S g}$. & $\mathbf{1 . ~ P l . ~}$ & MAN/ONE \\
\hline \multirow{4}{*}{ E } & qualitativ & 193006 & 446 & 150 & 75 \\
\cline { 2 - 6 } & quantitativ & 106663 & 450 & 85 & 30 \\
\cline { 2 - 6 } & $G^{2}$ & - & $\mathbf{8 0 , 0 4}$ & $\mathbf{0 , 6 7}$ & $\mathbf{1 , 2 4}$ \\
\hline \multirow{3}{*}{$\mathrm{D}$} & qualitativ & 16767 & 9 & 16 & 75 \\
\cline { 2 - 6 } & quantitativ & 113112 & 52 & 18 & 176 \\
\cline { 2 - 6 } & $\mathbf{G}^{\mathbf{2}}$ & - & $\mathbf{0 , 1 8}$ & $\mathbf{2 3 , 4 7}$ & $\mathbf{4 9 , 5 9}$ \\
\hline
\end{tabular}

Tabelle 5.12: Vergleich nach Forschungsansatz

Wie Tabelle 5.12 zeigt, deutet der $\mathrm{G}^{2}$-Wert hier für das englische Korpus nur bei der ersten Person Singular auf einen Unterschied dahingehend hin, dass sie im qualitativen Teilkorpus weniger häufig benutzt wird als im quantitativen. Für die deutschen Texte scheint es dagegen einen Unterschied zwischen qualitativen und quantitativen Texten beim Gebrauch von WIR und MAN zu geben, die beide häufiger in den qualitativen Texten verwendet wurden.

Die letzte Variable, die mit dem Log-Likelihood-Test überprüft werden soll, ist der akademische Status, der hier als Funktion der Schreiberfahrung gewertet wird. Je höher der akademische Status, desto mehr Erfahrung hat der entsprechende Autor wahrscheinlich mit dem wissenschaftlichen Schreiben. Dabei muss natürlich berücksichtigt werden, dass die Schreiberfahrung, genauso wie beispielsweise das Dienstalter, nicht notwendigerweise mit einem höheren akademischen Status einhergehen muss. Andersherum kann man aber wohl mit einiger Sicherheit davon ausgehen, dass jemand, der einen hohen akademischen Status erreicht hat, notwendigerweise auch vergleichsweise viel Schreiberfahrung hat.

\begin{tabular}{|l|l|l|}
\hline & englisch & deutsch \\
\hline $\begin{array}{l}\text { Graduate/nicht promo- } \\
\text { viert }\end{array}$ & 1 & 6 \\
\hline $\begin{array}{l}\text { Assistant } \\
\text { Prof./promoviert }\end{array}$ & 15 & 10 \\
\hline $\begin{array}{l}\text { Associate } \\
\text { Prof./habilitiert (PD) }\end{array}$ & 3 & 4 \\
\hline Full Professor/Professor & 6 & 5 \\
\hline
\end{tabular}

Tabelle 5.13: Verteilung der Texte nach Statuspositionen bzw. Schreiberfahrung 


\begin{tabular}{|c|c|c|c|c|c|c|}
\hline & \multicolumn{2}{|c|}{ 1. Sg. } & \multicolumn{2}{|c|}{ 1. Pl. } & \multicolumn{2}{|c|}{ MAN } \\
\hline & E & D & E & D & E & D \\
\hline $\begin{array}{l}\text { nicht } \\
\text { promo- } \\
\text { viert } \\
\text { Graduate }\end{array}$ & 61 & 4 & 5 & 2 & 9 & 46 \\
\hline $\begin{array}{l}\text { promo- } \\
\text { viert } \\
\text { assistant } \\
\text { prof. }\end{array}$ & 710 & 41 & 122 & 12 & 77 & \begin{tabular}{|l}
109 \\
\end{tabular} \\
\hline $\begin{array}{l}\text { Privatdo- } \\
\text { zent } \\
\text { associate } \\
\text { prof. }\end{array}$ & 120 & 6 & 8 & 10 & 6 & 28 \\
\hline $\begin{array}{l}\text { Professor } \\
\text { Full Pro- } \\
\text { fessor }\end{array}$ & 200 & 54 & 150 & 11 & 27 & 117 \\
\hline
\end{tabular}

Tabelle 5.14: Verteilung der Pronomen nach Status

\begin{tabular}{|l|l|l|l|}
\hline & 1. Sg. & 1. Pl & unpers. ONE \\
\hline Graduate & 2,24 & 5,54 & 2,19 \\
\hline Assistant Prof. & 0,03 & 10,97 & 0,18 \\
\hline Associate Prof. & 0,17 & 22,73 & 4,32 \\
\hline Full Prof. & 1,74 & 86,38 & 0,55 \\
\hline
\end{tabular}

Tabelle 5.15: $\mathrm{G}^{2}$-Werte nach Schreiberfahrung Englisch

\begin{tabular}{|l|l|l|l|}
\hline & 1.Sg. & $\mathbf{1 . P 1 .}$ & unpers. ONE \\
\hline nicht promoviert & 20,44 & 4,99 & 4,93 \\
\hline promoviert & 0,43 & 0,58 & 3,01 \\
\hline PD/habilitiert & 10,54 & 1,55 & 13,07 \\
\hline Professor & 38,99 & 3,03 & 53,28 \\
\hline
\end{tabular}

Tabelle 5.16: $\mathrm{G}^{2}$-Werte nach Schreiberfahrung Englisch

Beim Vergleich der unterschiedlichen akademischen Statuspositionen haben sich zwar nicht für jede Messung $\mathrm{G}^{2}$-Werte ergeben, die auf Unterschiede hindeuten, dennoch lassen sich einige interessante Tendenzen ablesen. Hier wurden die Texte der einzelnen Statuskategorien jeweils mit dem englischen bzw. deutschen Korpus insgesamt 
verglichen, die Ergebnisse sind dann entsprechend auch im Vergleich zum jeweiligen Gesamtkorpus zu interpretieren.

Für den Gebrauch der ersten Person Singular haben sich innerhalb der englischen Texte keine Unterschiede ergeben, interessanterweise deutet die Ausrichtung der Unterschiede, die der Log-liklihood-Calculator von Paul Rayson ergeben hat 15 , darauf hin, dass der Gebrauch mit steigendem Status und Schreiberfahrung abnehme. Dies ist jedoch im Rahmen eines so kleinen Korpus wie dem hier untersuchten nicht zuverlässig ablesbar. Es wäre aber sicherlich interessant, diese Tendenz an einer größeren Datenmenge zu überprüfen. Im Deutschen ergibt sich dagegen sogar das Ergebnis, dass in den Texten von Professoren häufiger das ICH verwendet wurde als im Gesamtkorpus ( $p<0,0001)$, während die Texte aller anderen Statusgruppen teils mit aussagekräftigen $\mathrm{G}^{2}$-Werten (Habilitierte mit $\mathrm{p}<0,01$, nicht Promovierte mit $\mathrm{p}<0,0001$ ) weniger ICHs aufwiesen als das gesamte deutsche Korpus.

Für die erste Person Plural zeigt sich im englischen Korpus dann eine ähnliche Verteilung wie für das ICH im deutschen. Auch hier hat sich ein hoher Wert dafür ergeben, dass die Full Professors im Vergleich zum gesamten Korpus das WIR bzw. WE häufiger benutzen. Alle anderen Statusgruppen benutzen die erste Person Plural deutlich weniger. Im deutschen Korpus hat sich für das WIR nur ein Wert von $p<0,05$ dahingehend ergeben, dass die nicht promovierten Autoren weniger häufig das WIR benutzen, als es im gesamten Korpus auftritt. Die generelle Tendenz scheint aber auch hier zu sein, dass der Gebrauch dieses Pronomens mit zunehmender Schreiberfahrung ebenfalls zunimmt.

Für das unpersönliche Pronomen ist im Deutschen eine ähnliche Tendenz erkennbar, auch hier ist der Gebrauch in den ersten drei Statusgruppen verglichen mit dem Gesamtkorpus niedriger, wobei sich für die nicht Promovierten der Einfluss des akademischen Status auf den Gebrauch des Pronomens mit $\mathrm{p}<0,05$ und für die Habilitierten mit $\mathrm{p}<0,001$ vermuten lässt. Die Professoren gebrauchen das MAN dagegen deutlich häufiger als es im gesamten Korpus vorkommt ( $<<0,0001)$. Für das Englische ließ sich dagegen nur zeigen, dass die Associate Professors das ONE verglichen mit dem gesamten englischen Korpus weniger benutzten $(p<0,05)$.

\subsubsection{Vergleich nach Rollen}

\subsubsection{Vergleich 1. Person Singular}

Nach diesem Überblick über die Verteilung der Pronomen im Allgemeinen folgt nun eine tiefer gehende Analyse in Hinblick auf die einzelnen Rollen und die diese Rollen symbolisierenden Handlungen. Dazu wird einerseits wieder die quantitative Perspektive betrachtet, indem die Verteilung der einzelnen Rollenhandlungen in prozentualen

\footnotetext{
${ }^{15}$ s. Fußnote auf S. 144
} 
Bezug einerseits innerhalb der drei großen Rollenkategorien Verfasser, Forscher und Andere und andererseits in Bezug zu der Gesamtmenge des jeweiligen Pronomens gesetzt wird. Der Fokus liegt jedoch auf der qualitativen Auswertung der einzelnen Handlungskategorien. Der Text von Diane Vaughan (Vaughan 2006) wird weiterhin durchgängig nicht berücksichtigt. Die für das englische Korpus genannten Zahlen beziehen sich hier also immer auf 24 Texte ${ }^{16}$. Der ICH-Gebrauch im Text von Vaughan wird in Kap. 5.3 näher betrachtet.

\subsection{Verfasser}

Unter die Verfasserhandlungen fallen, wie schon in Kap 5.1.1.1 beschrieben, diejenigen Handlungen, die auf den Text bezogen, d. h. textintern sind. Diese Handlungen stellen im englischen Korpus in insgesamt 24 Texten 24,29\% aller Handlungen, die das ICH ausführt, und im deutschen in nur neun Texten sogar 44,76 \% (s. Tab. 5.20). Hier zeigt sich der erste deutliche Unterschied zwischen den beiden Korpora. Dennoch ergibt die Überprüfung mit dem Log-Likelihood-Test immer noch eine deutliche Tendenz, dass im Englischen die meisten dieser Verfasserhandlungen häufiger benutzt werden als im Deutschen. Für die Handlungen des verbalen und graphischen Darstellens $\left(\mathrm{G}^{2}=\right.$ $30,32)$ und der Klärung der Terminologie $\left(G^{2}=25,41\right)$ übersteigt der $\mathrm{G}^{2}$-Wert sogar den Wert von 15,13 für $\mathrm{p}<0,0001$. Damit stützt der Test die Annahme, dass im Englischen mehr dieser Verfasserhandlungen vorkommen als im Deutschen. Dies gilt auch, wenn man die Verfasserhandlungen insgesamt betrachtet $\left(G^{2}=58,9\right)$.

Der $\mathrm{G}^{2}$-Wert für $\mathrm{p}<0,01,6,63$, wird überschritten, wenn man die Handlungen vergleicht, mit denen das Ziel oder der Fokus des Textes dargestellt wird $\left(G^{2}=8,13\right)$. Nur für das Strukturieren des Textes $\left(G^{2}=1,25\right)$ und andere metakommunikative Handlungen $\left(G^{2}=1,42\right)$ konnte kein Effekt bezüglich der Sprache festgestellt werden, da sie selbst den Wert für $\mathrm{p}<0.05,3,84$, nicht überschritten. Die folgenden Tabellen 5.17 bis 5.20 geben darüber hinaus einen Überblick über die prozentuale Verteilung der Verfasserhandlungen.

\footnotetext{
${ }^{16} \mathrm{Im}$ englischen Korpus gab es 30 Vorkommnisse der ersten Person Singular, die nicht eindeutig kategorisiert werden konnten und auf die hier nicht näher eingegangen wird. Im deutschen Korpus konnten alle 105 Vorkommen der ersten Person Singular kategorisiert werden.
} 


\begin{tabular}{|l|l|l|l|l|}
\hline Rolle & $\begin{array}{l}\text { Englisch } \\
\text { absolut }\end{array}$ & $\begin{array}{l}\text { Deutsch abso- } \\
\text { lut }\end{array}$ & gesamt & $\mathbf{G}^{\mathbf{2}}$ \\
\hline $\begin{array}{l}\text { Verfasser } \\
\text { gesamt }\end{array}$ & 265 & 47 & 312 & 58,9 \\
\hline $\begin{array}{l}\text { verbale oder } \\
\text { graphische } \\
\text { Darstellung }\end{array}$ & 139 & 25 & 164 & 30,32 \\
\hline $\begin{array}{l}\text { Fokus des } \\
\text { (Teil-)Textes }\end{array}$ & 35 & 6 & 41 & 8,13 \\
\hline $\begin{array}{l}\text { Terminologie- } \\
\text { klärung }\end{array}$ & 50 & 3 & 53 & 25,41 \\
\hline $\begin{array}{l}\text { Textstruktu- } \\
\text { rierung }\end{array}$ & 19 & 7 & 25 & 1,25 \\
\hline $\begin{array}{l}\text { sonstige } \\
\text { Metakommu- } \\
\text { nikation }\end{array}$ & 22 & 6 & 29 & 1,42 \\
\hline
\end{tabular}

Tabelle 5.17: Übersicht Verfasserhandlungen absolut

\begin{tabular}{|l|l|l|l|}
\hline Rolle & Englisch & Deutsch & gesamt \\
\hline Verfasser gesamt & 24 & 9 & 33 \\
\hline $\begin{array}{l}\text { verbale oder } \\
\text { graphische Dar- } \\
\text { stellung }\end{array}$ & 23 & 6 & 29 \\
\hline $\begin{array}{l}\text { Fokus des (Teil-) } \\
\text { Textes }\end{array}$ & 14 & 3 & 17 \\
\hline $\begin{array}{l}\text { Terminologie- } \\
\text { klärung }\end{array}$ & 14 & 2 & 16 \\
\hline Textstrukturierung & 13 & 5 & 18 \\
\hline $\begin{array}{l}\text { sonstige Meta- } \\
\text { kommunikation }\end{array}$ & 12 & 4 & 16 \\
\hline
\end{tabular}

Tabelle 5.18: Verteilung der Verfasserhandlungen auf Texte 


\begin{tabular}{|l|l|l|}
\hline Rolle & Englisch & Deutsch \\
\hline Verfasser gesamt & $\mathbf{1 0 0}$ & $\mathbf{1 0 0}$ \\
\hline $\begin{array}{l}\text { verbale oder graphische } \\
\text { Darstellung }\end{array}$ & 52,45 & 53,19 \\
\hline Fokus des (Teil-)Textes & 13,21 & 12,77 \\
\hline Terminologieklärung & 18,87 & 6,38 \\
\hline Textstrukturierung & 7,17 & 12,77 \\
\hline $\begin{array}{l}\text { sonstige Metakommuni- } \\
\text { kation }\end{array}$ & 8,30 & 14,89 \\
\hline
\end{tabular}

Tabelle 5.19: prozentuale Verteilung der Verfasserhandlungen auf die Verfasserrolle

\begin{tabular}{|l|l|l|}
\hline Rolle & Englisch & Deutsch \\
\hline Verfasser gesamt & $\mathbf{2 4 , 2 9}$ & $\mathbf{4 4 , 7 6}$ \\
\hline $\begin{array}{l}\text { verbale oder graphische } \\
\text { Darstellung }\end{array}$ & 12,74 & $\mathbf{2 3 , 8 1}$ \\
\hline Fokus des (Teil-)Textes & 3,21 & 5,71 \\
\hline Terminologieklärung & 4,58 & 2,86 \\
\hline Textstrukturierung & 1,74 & 5,71 \\
\hline $\begin{array}{l}\text { sonstige Metakommuni- } \\
\text { kation }\end{array}$ & 2,02 & 6,67 \\
\hline
\end{tabular}

Tabelle 5.20: prozentuale Verteilung der Verfasserhandlungen auf die gesamte 1. Person Singular

\section{Verbale und graphische Darstellungen}

Diese Kategorie ist die größte und umfassendste der Verfasserhandlungen und sie enthält sowohl im englischen als auch im deutschen Korpus etwas über die Hälfte aller dieser Handlungen, d. h. 52,45 \% (139) in den englischen bzw. 53,19 \% (25) in den deutschen Texten (s. Tabellen 5.19, S. 151 und 5.20, S. 151). Dabei verteilen sich diese Vorkommen auf 23 englische bzw. sechs deutsche Texte. Auf die gesamte erste Person Singular gerechnet zeigt sich aber ein deutlicher Unterschied. Während fast ein Viertel $(23,81 \%)$ aller deutschen ICHs mit Handlungen dieser Art kombiniert werden, sind es im englischen Korpus nur umgerechnet etwa ein Achtel (12,74 \%, s. Tabelle 5.20). Die amerikanischen Autoren schreiben sich also nur halb so häufig Handlungen der verbalen und graphischen Darstellung zu wie die deutschen und bevorzugen entsprechend andere Handlungen stärker.

Unter diese Verfasserhandlungen fallen einerseits Handlungen der sprachlichen Äußerung, wie z. B. say, speak, explain, discuss, erläutern etc. Dies beinhaltet auch Verbkomplexe wie auf eine Frage eingehen, to address an issue etc. Daneben enthält diese Kate- 
gorie auch äquivalente Handlungen der graphischen bzw. visuellen Darstellung, wie zeigen bzw. show, skizzieren, illuminate, illustrate etc. Die Verteilung dieser beiden Perspektiven ist in beiden Sprachen etwa gleich, d. h. es gibt etwa gleich viele verbale und graphisch-visuelle Handlungen.

(157) I paint the picture of baseline facts with a broad brush because lucid accounts have filled in the details elsewhere, including not just the two articles in question but also prior reviews (notably, Goering and Feins 2003; Orr et al. 2003; Kling et al. 2007) (Sampson 2008)

(158) Diesbezügliche Stärken und Schwächen von Lebensstiltypologien möchte ich an einem Anwendungsbeispiel illustrieren, das verschiedentlich als vorbildlich für seine Leistungsfähigkeit erachtet wird. (Otte 2005)

(159) In the next section, I describe the analysis framework, by means of which "participation shifts" - transitions in who is speaking and/or who is being addressed-are exhaustively inventoried as the first step toward quantitative analysis, eventually of the degree to which pairs of individuals involved in particular participation shifts are also tied in one or more underlying networks. (Gibson 2005)

(160) Auf der Basis einer Auswertung einer Eurobarometerbefragung habe ich in einem ersten Schritt die Intensität der Hochkulturnutzung in 27 Ländern der Europäischen Union vergleichend beschrieben. (Gerhards 2009)

Mit diesen Handlungen beschreibt der Autor einfach, was er in seinem Text tut. Dies stellt er dem Leser grundsätzlich dar, die benutzten Verben beschreiben im Allgemeinen Handlungen, die nur der Autor selber ausführt und ausführen kann. Der Leser wird somit praktisch „,vor vollendete Tatsachen gestellt" bzw. erhält einen Bericht darüber, wie der Autor in seinem Text weiter vorgeht, ohne (semantisch) die Möglichkeit einer Auswahl zu haben. Da bei den hier verwendeten Handlungen jedoch nur eine sehr geringe Gefahr der Gesichtsbedrohung besteht, sind sie entsprechend wenig risikobehaftet (s. z. B. Myers 1989: Hyland 2002a).

In den englischen Texten wurde daneben allerdings dennoch eine kleine Untergruppe an Verben benutzt, die dem Leser von ihrer Bedeutung her diese Auswahl anbieten, vor allem die Verben offer und propose.

(161) Here I offer three primary dimensions of political opportunity structure at the transnational level that explain how power is established at the transnational level: (1) the constitution of transnational actors and interests, (2) the definition and recognition of transnational rights, and (3) adjudication of rights at the transnational level. (Kay 2005)

(162) I then propose a conceptual framework that posits the intrinsically dramaturgical quality of political interaction in a state socialist context and discuss the 
implications of this approach for the interpretation of evidence about political activities under state socialist conditions. (Straughn 2005)

Diese Formulierung gibt dem Leser zumindest semantisch die Möglichkeit die angebotenen Claims, zu denen der Autor seine Leser an diesen Stellen hinleitet, auch abzulehnen. Dadurch wird der Leser sprachlich in die Argumentation mit einbezogen, wodurch der Autor möglichen Face-Threats bzw. Kritik an den dargebotenen Claims begegnet. Dies entspricht nach Ken Hylands Kategorisierung von Stance und Engagement eher einer Engagement-Strategie. Eine ähnliche Strategie wenden auch die deutschen Autoren an, indem sie ihre verbalen und graphischen Darstellungen in der Hälfte aller beobachteten Fälle mit den Modalverb möchten kombinieren und damit abschwächen ${ }^{17}$ In den englischen Beispielen kommt das äquivalente „I would like to“ nur einmal vor.

(163) Daher möchte ich abschließend skizzieren, wie eine Neuorientierung der typologischen Lebensstilforschung aussehen könnte, die die Voraussetzungen für eine systematischere empirische Untersuchung ihrer zentralen Behauptungen schafft. (Otte 2005)

(164) Diese Zusammenhänge möchte ich etwas genauer am Beispiel der Ausbreitung von McDonalds in Ostasien erläutern. (Schwinn 2006)

(165) Before considering its methodological implications, I would like to emphasize the inherently social dimension of this model. (Vaisey 2009)

Dies ist kohärent mit den weiteren Beobachtungen, dass im Deutschen der wissenschaftliche Autor noch deutlich stärker im Hintergrund steht als im Englischen und dass die wissenschaftliche Persona entsprechend vorsichtig und zurückhaltend handeln muss, um das Face der an der Kommunikation Beteiligten zu schützen.

\section{Darstellen des Ziels bzw. des Fokus des (Teil-)Textes}

Das Darstellen des Textziels bzw. -fokus ist eine weitere Verfasserhandlung, die in beiden Sprachen in ähnlichem Ausmaß vollführt wird (Englisch: 13,21 \%, Deutsch: $12,77 \%$ aller Verfasserhandlungen in 14 bzw. drei Texten, s. Tabellen 5.18 bis 5.20). Auf die gesamte erste Person Singular gerechnet ergibt sich hier ein nur ein kleiner Unterschied von 3,21 \% im englischen Korpus gegenüber 5,71 \% im deutschen. Hier werden vor allem Verben wie examine (5), explore (5), be concerned with (4), focus (4) verwendet. In den fünf deutschen Beispielen kommt sich konzentrieren auf zweimal vor, die anderen Verben bzw. Verbkomplexe jeweils nur einmal: untersuchen, (benutzen und) prüfen und (nicht) den Anspruch erheben.

\footnotetext{
${ }^{17}$ Die Verwendung von Modalverben im Allgemeinen, ebenso wie die Verwendung unterschiedlicher Zeitformen (die metakommunikative Funktion der Verfasserrolle wird beispielsweise häufig auch dadurch verstärkt, dass die Handlungen im Perfekt beschrieben werden) sind sicherlich weitere lohnende Untersuchungsfokusse, die aber in dem hier gegebenen Rahmen nicht weiter verfolgt werden.
} 
(166) First, however, I explore how organizing became institutionalized through the NLRB and examine the movement toward more contentious modes of action. Although specific to labor, the processes described have parallels across a range of contentious activities. (Martin 2008)

(167) Here I examine one transnational relationship that emerged among many in the struggle against NAFTA that together constituted a transnational social movement; elsewhere I provide a more detailed discussion (...). (Kay 2005)

(168) Ich werde im Folgenden die Hochkulturorientierung der Bürger in den 27 Mitgliedsländern der Europäischen Union vergleichend untersuchen. (Gerhards 2009)

(169) In diesem Abschnitt konzentriere ich mich auf den ersten Aspekt. (Schwinn 2006)

Diese Handlungen werden im Kotext immer durch Zusätze wie here, in this article, im Folgenden etc. auf den vorliegenden Text eingegrenzt. Dies unterscheidet diese Kategorie von der Forscher-Kategorie Fokussierung der Forschungsfrage, in der es um den übergeordneten Forschungsfokus geht, der nicht auf den Text beschränkt ist.

Eine weitere Handlung, die ebenfalls in diese Kategorie fällt, ist die Darstellung des theoretischen Hintergrunds mit direktem Bezug zum Text. Auch diese Kategorie hat ein Äquivalent in der Forscher-Rolle, wo der theoretische Hintergrund der gesamten Untersuchung erläutert wird. Bei den acht englischen Vorkommnissen, die zur VerfasserRolle zählen, ist aber der Bezug zum Text eindeutig sprachlich dargestellt. Der Autor konstruiert sich also deutlich als Verfasser, der im Rahmen der Textproduktion dem Leser die notwendigen Hintergründe bereitstellt und entsprechend eine metakommunikative Handlung ausführt, und nicht als Forscher, der weitreichende Entscheidungen über theoretische Grundpositionen trifft.

(170) Before describing these data and moving on to the analysis, I first orient my research in the literature on neoinstitutional theory and state social control, particularly work on punishment as related to politics, religion, and race. (King 2008)

(171) In the following section, I situate my work in the current research on the role of education in social stratification. (Madsen 2007)

\section{Terminologieklärung}

Diese Handlungen werden deutlich häufiger von amerikanischen Autoren mit der ersten Person Singular kombiniert, 18,87 \% aller Verfasserhandlungen in 14 englischen Texten fallen in diese Kategorie. Demgegenüber handeln nur 6,38 \% der deutschen ICHs terminologieklärend, was auch nur in zwei Texten auftritt. Auf den Gebrauch 
der ersten Person Singular insgesamt gerechnet fallen mit 4,58 \% auch knapp doppelt so viele englische Is wie deutsche ICHs $(2,86 \%)$.

Hierunter werden v. a. diejenigen Handlungen gefasst, mit denen die Autoren ihren Lesern die von ihnen benutzte Terminologie erklären, Begriffe definieren und diejenigen Konzepte aus dem bestehenden fachlichen Diskurs auswählen, die für ihre Forschung bzw. konkret für diesen Text relevant und passend sind. Die Textbezogenheit wird häufig auch im weiteren Kotext der verbalen Handlung durch metakommunikative Zusätze wie throughout the article oder hier (Beispiele 173 und 176) markiert. Wichtig ist, dass die Terminologie, die erklärt und definiert wird, im fachlichen Diskurs bereits vorhanden ist und hier vom Autor nur übernommen wird. Demgegenüber wird die Begriffsbildung, d.h. das Prägen neuer Begrifflichkeiten zu der Forscherhandlung des Aufstellen neuer Claims gezählt (s. Kap 5.2.2.1.2, S. 168.

Die wichtigsten Verben, mit denen die erste Person Singular hier kombiniert wird, sind im Englischen use (the term/the phrase/etc), refer, define und seltener auch label, denote, call, characterize, (understand to) mean. Im Deutschen sind die ICHs häufig diejenigen, die bezeichnen oder von etwas sprechen.

(172) NAFTA is more accurately a regional governance institution, but for simplicity and consistency with the term used in the literature, I will refer to it as a global governance institution. (Kay 2005)

(173) Throughout the article, I use institutional preferences for race/ethnicity and affirmative action interchangeably.

(174) I use the term "northern" to designate states outside the South, which I define, following V. O. Key (1949), as the 11 states belonging to the former Confederacy. (Chen 2007)

(175) When a person speaks after he or she is addressed, I refer to it as "turn receiving," since he or she is on the receiving end of a transfer initiated by the prior speaker. (Gibson 2005)

(176) Ich bezeichne hier mit Kultur den gesellschaftlichen Bereich, der sich auf Kunstaktivitäten im weitesten Sinne bezieht. (Gerhards 2009)

\section{Textstrukturierung}

Die Textstrukturierung ist ein wesentlicher Bestandteil beim Schreiben und kann mit diversen sprachlichen Mitteln realisiert werden. Diese Kategorie jedoch ist sehr eng gefasst und enthält zumindest im englischen Korpus hauptsächlich diejenigen Sätze, deren Verben direkt die Strukturierung und den Textaufbau beschreiben. Dies sind vor allem begin und conclude in den englischen Texten. Andere, nur einmalig vorkommende textstrukturierende Handlungen, sind z. B. summarize bzw. zusammenfassen und divide. 
Während im deutschen Korpus 12,77 \% der Verfasserhandlungen in diese Kategorie fallen, sind es im englischen nur 7,17 \%. Auf die gesamte erste Person Singular gerechnet liegt die Verteilung bei 5,71 \% gegenüber 1,74\%. Dabei verteilen sich die Vorkommen auf 13 englische und fünf deutsche Texte.

(177) I begin by reviewing the fiscal-military and rational choice models of state formation and Kennedy's (1987) theory of "imperial overstretch" (p. 515). (Lachmann 2009)

(178) I conclude with a summary of my historical findings and discuss more general implications of a legal framing perspective for theory and research on cultural framing processes and social movements. (Pedriana 2006)

(179) I have divided this article into six principal sections.

(180) Vielmehr möchte ich die Ergebnisse mit folgender Aussage zusammenfassen: (...). (Brose 2008)

Die anderen beiden Beispiele aus dem deutschen Korpus sind nicht ganz so eindeutig nur am Verb zu identifizieren, hier ist also wieder der Kotext entscheidend für die Einordnung in diese Kategorie. Dennoch ist es deutlich, dass auch diese Sätze der Textstrukturierung dienen, was in Beispiel 181 am Zusatz zunächst und in Beispiel 182 an den Verweisen auf verschiedene Abschnitte des Textes deutlich wird. Die hier verwendeten Verben nachgehen und vornehmen bzw. herausarbeiten sprechen deutlich für die Verfasserrolle, sind aber für sich allein genommen nicht eindeutig in eine der anderen Verfasserkategorien einzuordnen. Grundsätzlich wäre es aber auch in den deutschen Texten vorstellbar, wie im Englischen die Verben beginnen und schließen in dieser Funktion $\mathrm{zu}$ verwenden.

(181) Diesen Anweisungen und der vorstehenden Modellskizze folgend, werde ich zunächst einer globalen Konstellation nachgehen, um daraus die kulturellen Konsequenzen abschätzen zu können. (Schwinn 2006)

(182) Über eine Bilanz hinaus, die ich in neun Unterpunkten in Abschnitt II vornehme, möchte ich in Abschnitt III Implikationen für die Ausgestaltung einer zukünftigen Lebensstilforschung herausarbeiten. (Otte 2005)

Als Unterkategorie der Textstrukturierung können Vor- und Rückverweise gesehen werden. Hier werden ebenfalls nur diejenigen Sätze eingeordnet, bei denen der Verweis im Verb realisiert wird und es sich somit um die textinhärente Handlung handelt. Die im Korpus nachgewiesenen Verben sind return (viermal), revisit, zurückkehren und zurückkommen (jeweils einmal).

(183) Future work could gain additional leverage in determining the sufficiency of these conditions by adding negative or "failed" cases-a topic to which I return in the conclusion. (Bartley 2007) 
(184) What we mean empirically by "neighborhood" cannot be ignored, however; I revisit this issue later. (Sampson 2008)

(185) Zum Abschluss möchte ich noch einmal zu Max Webers Charisma-Theorie zurückkehren, um den Legitimationszugewinn, der mit den Selig-und Heiligsprechungen verbunden ist, durch eine erweiterte Theorie des Charismas zu erklären und damit das herrschaftssoziologische "Geheimnis" der Selig-und Heiligsprechungen ein wenig zu lüften. (Bienfait 2006)

(186) Ich komme auf das für die Fragestellung des Artikels wichtige Verhältnis zwischen inkorporiertem kulturellem Kapital, Geschmack, Habitus, Kunstorientierung und Lebensstilen gleich nochmals genauer zurück. (Gerhards 2009)

\section{Sonstige Metakommunikation}

In diese Kategorie fallen alle diejenigen Handlungen, mit denen der Autor seinen Text auf eine Weise kommentiert, die nicht einer der anderen Verfasserhandlungen entspricht. Vorrangig fallen hierunter Kommentare und Bemerkungen zu Auswahl und Aufbau des Textes sowie der Inhalte, insbesondere auch Begründungen, warum etwas nicht behandelt wird. Im englischen Korpus macht diese Kategorie 8,30 \% der Verfasserhandlungen und 2,02 \% aller Vorkommen der ersten Person Singular aus und ist nach der Textstrukturierung die Handlungskategorie, die am seltensten auftritt. Dennoch wurde sie in 12 der hier zugrunde gelegten 24 Texte gefunden. In den deutschen Texten hingegen ist diese Handlungskategorie nach der verbalen und graphischen Darstellung die zweitgrößte Handlungskategorie und enthält 14,89 \% der Verfasserhandlungen sowie 6,67 \% aller ICH-Vorkommen in vier Texten.

Aufgrund der besonderen Diversität dieser Kategorie können hier keine typischen Verben angegeben werden. Es ist jedoch auffällig, dass in den englischen Texten besonders häufig phrasal verbs wie set aside, dwell upon, take up benutzt werden.

(187) Table 1 presents a complete inventory of those participation shifts, or "P-shifts," that occur over the space of two speaking turns; for the purposes of this article, I set aside those that involve a change of target but not of speaker. (Gibson 2005)

(188) It is an open question, and one I do not take up here, of whether we are now moving into some third stage, characterized by the declining legitimacy of the modern state with a concomitant dissolution of the state's symbolic power. (Loveman 2005)

(189) Vgl. auch die von einem strikt reduktionistischen Standpunkt ausgehende Kritik am moderaten methodologischen Holismus unter Berücksichtigung Max Webers von Jens Greve (2006b), auf die ich hier aus Platzgründen leider nicht eingehen kann. (Albert 2005) 
(190) Da sich unsere Daten aber allein auf eine Hochkulturorientierung beziehen, die so genannte „Allesfresserthese" aber unterschiedliche Kulturaktivitäten (Hochund Populärkultur) einschließt, lasse ich diese These hier unberücksichtigt. (Gerhards 2009)

Darüber hinaus werden hier auch Handlungen subsumiert, mit denen die Autoren ihr eigenes Schreibverhalten (im weitesten Sinne) kommentieren.

(191) I tend to cite the published industry (and government) sources rather than specific interviews in order to provide a readily verifiable basis for my claims without compromising my commitment to respondents who requested confidentiality. (Schrank 2010)

(192) Lest I be misunderstood, let me clarify what I have not done. (Vaisey 2009)

(193) Offensichtlich habe ich diesen Begriff an genannter Stelle aber eingeführt, um etwas grundlegend anderes zu bezeichnen. (Albert 2005)

Zusammenfassend kann man also eindeutig sagen, dass die deutschen Autoren, wenn sie denn die erste Person Singular überhaupt benutzen, sich deutlich häufiger in ihrer Rolle als Verfasser des Textes darstellen als die amerikanischen Autoren. Während im englischen Korpus rund ein Viertel (24,29 \%) aller Is Verfasserhandlungen zugeschrieben wurden, sind es im deutschen Korpus beinahe die Hälfte aller ICHs (44,76 \%), die als Agens bzw. Subjekt für diese Handlungen fungieren. Einzig beim Klären der benutzten Terminologie referieren die englischsprachigen Autoren häufiger auf sich selbst als diejenigen, die Definitionen aus den vorhandenen Diskurs auswählen und über den Gebrauch ihrer Begriffe entscheiden.

\subsection{Forscher}

Unter den Forscherhandlungen werden alle Handlungen zusammengefasst, die sich nicht nur auf den Schreibprozess beziehen, sondern das im weiteren Sinne „wissenschaftliche“ Arbeiten beschreiben (s. Kap. 5.1.1.2). Im englischen Korpus fallen 71,86 \% aller Handlungen, die in der ersten Person Singular realisiert werden, in diese Kategorie, im deutschen sind es dagegen nur 45,71\%, wobei sich diese auf 24 bzw. 14 Texte verteilen. Der Log-Liklihood-Test zeigt, nicht überraschend, dass die Forscherhandlungen fast immer häufiger im Englischen in der ersten Person Singular benannt werden als im Deutschen. Ein Wert von $\mathrm{p}<0,0001$ ergab sich dabei für die Handlungen Fokussieren der Forschungsfrage, Datenerhebung, Datenaufbereitung/-analyse, Ergebnisse, Argumentation/Arguer sowie auch beim Gesamtvergleich.

Für die Rolle des Originators (Aufstellen neuer Claims) konnte noch $\mathrm{p}<0,001$ nachgewiesen werden, der $\mathrm{p}$-Wert für das Danksagen liegt bei $\mathrm{p}<0,5$. Für die Kategorien $A u f$ stellen von Hypothesen und Grundannahmen $\left(\mathrm{G}^{2}=1,86\right)$ und andere Forschungshandlungen $\left(G^{2}=3,75\right)$ konnten keine nennenswerten Effekte festgestellt werden. Die folgenden 
Tabellen 5.21 bis 5.24 geben darüber hinaus einen Überblick über die prozentuale Verteilung der Forscherhandlungen.

\begin{tabular}{|l|l|l|l|l|}
\hline Rolle & $\begin{array}{l}\text { Englisch } \\
\text { absolut }\end{array}$ & $\begin{array}{l}\text { Deutsch abso- } \\
\text { lut }\end{array}$ & gesamt & $\mathrm{G}^{2}$ \\
\hline $\begin{array}{l}\text { Forscher } \\
\text { gesamt }\end{array}$ & 784 & 48 & 832 & 394,92 \\
\hline $\begin{array}{l}\text { Fokussierung } \\
\text { der For- } \\
\text { schungsfrage }\end{array}$ & 53 & 1 & 54 & 36,75 \\
\hline $\begin{array}{l}\text { Hypothesen } \\
\text { und Grundan- } \\
\text { nahmen }\end{array}$ & 38 & 13 & 51 & 1,86 \\
\hline Datenerhebung & 156 & 0 & 156 & 131,19 \\
\hline $\begin{array}{l}\text { Datenauf- } \\
\text { bereitung/ } \\
\text {-analyse }\end{array}$ & 281 & 9 & 290 & 175,32 \\
\hline Ergebnisse & 36 & 0 & 36 & 30,27 \\
\hline $\begin{array}{l}\text { Argumentation } \\
\text { Arguer }\end{array}$ & 123 & 6 & 129 & 67,73 \\
\hline Originator & 36 & 4 & 40 & 12,82 \\
\hline Danksagung & 41 & 11 & 52 & 4,34 \\
\hline andere FH & 20 & 4 & 24 & 3,75 \\
\hline
\end{tabular}

Tabelle 5.21: Übersicht Forscherrollen absolut 


\begin{tabular}{|l|l|l|l|}
\hline Rolle & Englisch & Deutsch & gesamt \\
\hline Forscher gesamt & 24 & 14 & 38 \\
\hline $\begin{array}{l}\text { Fokussierung der } \\
\text { Forschungsfrage }\end{array}$ & 19 & 1 & 20 \\
\hline $\begin{array}{l}\text { Hypothesen und } \\
\text { Grundannahmen }\end{array}$ & 18 & 3 & 21 \\
\hline Datenerhebung & 22 & 0 & 22 \\
\hline $\begin{array}{l}\text { Datenaufberei- } \\
\text { tung/ -analyse }\end{array}$ & 21 & 2 & 23 \\
\hline Ergebnisse & 10 & 0 & 10 \\
\hline $\begin{array}{l}\text { Argumentation/ } \\
\text { Arguer }\end{array}$ & 21 & 3 & 24 \\
\hline Originator & 15 & 3 & 18 \\
\hline Danksagung & 21 & 8 & 29 \\
\hline andere FH & 10 & 3 & 13 \\
\hline
\end{tabular}

Tabelle 5.22: Verteilung der Forscherhandlungen auf Texte

\begin{tabular}{|l|l|l|}
\hline Rolle & Englisch & Deutsch \\
\hline Forscher gesamt & $\mathbf{1 0 0}$ & $\mathbf{1 0 0}$ \\
\hline $\begin{array}{l}\text { Fokussierung der For- } \\
\text { schungsfrage }\end{array}$ & 6,76 & 2,08 \\
\hline $\begin{array}{l}\text { Hypothesen und Grund- } \\
\text { annahmen }\end{array}$ & 4,85 & 27,08 \\
\hline Datenerhebung & 19,90 & 0 \\
\hline $\begin{array}{l}\text { Datenaufbereitung/ } \\
\text {-analyse }\end{array}$ & 35,84 & 18,75 \\
\hline Ergebnisse & 4,59 & 0 \\
\hline Argumentation/ Arguer & 15,69 & 12,5 \\
\hline Originator & 4,59 & 8,33 \\
\hline Danksagung & 5,23 & 22,92 \\
\hline andere FH & 2,55 & 8,33 \\
\hline
\end{tabular}

Tabelle 5.23: prozentuale Verteilung der Forscherhandlungen auf die Forscherrolle 


\begin{tabular}{|l|l|l|}
\hline Rolle & Englisch & Deutsch \\
\hline Forscher gesamt & $\mathbf{7 1 , 8 6}$ & $\mathbf{4 5 , 7 1}$ \\
\hline $\begin{array}{l}\text { Fokussierung der For- } \\
\text { schungsfrage }\end{array}$ & 4,86 & 0,95 \\
\hline $\begin{array}{l}\text { Hypothesen und Grund- } \\
\text { annahmen }\end{array}$ & 3,48 & 12,38 \\
\hline Datenerhebung & 14,30 & 0 \\
\hline $\begin{array}{l}\text { Datenaufbereitung/ } \\
\text {-analyse }\end{array}$ & 25,76 & 8,57 \\
\hline Ergebnisse & 3,30 & 0 \\
\hline Argumentation/ Arguer & 11,27 & 5,71 \\
\hline Originator & 3,30 & 3,81 \\
\hline Danksagung & 3,76 & 10,48 \\
\hline andere FH & 1,83 & 3,81 \\
\hline
\end{tabular}

Tabelle 5.24: prozentuale Verteilung der Forscherhandlungen auf die gesamte 1. Person Singular

\section{Fokussieren der Forschungsfrage}

Das Formulieren der Forschungsfrage bzw. in den eher theoretisch ausgerichteten Texten des Desiderats sowie das Eingrenzen des (Forschungs-)Fokus sind Handlungen, die im Forschungsprozess sehr früh vollzogen werden müssen, meist auch lange bevor ein Artikel über das Projekt berichten kann. Insofern werden sie hier als textexterne Forscherhandlungen betrachtet, anders als die oben beschriebene Verfasserhandlung des Darstellens von Ziel und Fokus (s. Kap. 5.2.2.1.1). In dieser Kategorie werden diejenigen Handlungen berücksichtigt, die sich nicht nur auf den Text beschränken, sondern der gesamten Forschung zugrunde liegen. Dafür wurden im englischen Korpus insbesondere Verben wie focus (on), examine und be interested (in) verwendet, teilweise auch in negierter Form, um anzuzeigen, was gerade nicht untersucht wird.

Das Formulieren der Forschungsfrage bzw. das Eingrenzen des Forschungsfokus macht in den englischen Texten 6,76\% der Forscherhandlungen und 4,86 \% aller Handlungen der ersten Person Singular aus, in den deutschen fallen dagegen nur 2,08 \% der Forscherhandlungen und 0,95\% aller ICH-Handlungen in diese Kategorie. Die englischen Autoren schreiben sich diese Aufgabe also deutlich häufiger in der ICH-Form zu als die deutschen $\left(\mathrm{G}^{2}=36,75, \mathrm{p}<0,0001\right)$. Dies wird auch daran deutlich, dass 19 der amerikanischen Autoren diese Handlung verwenden, wohingegen sie in nur einem deutschen Text vorkam.

(194) I focus on the gray area between these legal categories, how this "in-between" status or liminal legality shapes different spheres of life-the immigrant's immediate sphere of social networks and family, the community-level place of re- 
ligious institutions in the immigrants' lives, and the broader domain of artistic expression. (Menjívar 2006)

(195) Because my methodological approach differs from that used in these influential social scientific studies, however, I emphasize certain dimensions of religious individualism that they have not clearly noticed. (Madsen 2009)

(196) I am not interested so much in the raw number of veto points but in which party controls any veto point-and particularly whether the GOP has control. (Chen 2007)

(197) Ich sehe in theoretisch-konzeptioneller Hinsicht insgesamt das Differenzierungsund Integrationserfordernis, die Verschränktheit von institutionellen Faktoren auf der nationalen Ebene einerseits und branchenspezifischen Veränderungsdynamiken andererseits zu identifizieren. (Teipe 2009)

\section{Auswählen von Hypothesen und Ausgangsannahmen sowie theoretischen Grundposi- tionen}

Mit den Handlungen, die zu dieser Kategorie gehören, werden die der Forschung bzw. der im Text dargelegten Überlegungen zugrundeliegenden Hypothesen vorgestellt. Im Gegensatz zur Darstellung des theoretischen Hintergrunds als Verfasserhandlung geht der Autor hier ein deutlich größeres Risiko ein. Er ist nicht einfach nur derjenige, der die Theorie darstellt, sondern derjenige, der sie auswählt bzw. sogar formuliert, wodurch er sich deutlich für mögliche Kritik öffnet.

Dafür werden in den englischen Texten vor allem Verben wie expect, hypothesize, assume und anticipate verwendet. Die deutschen Autoren stellen sich äquivalent als diejenigen dar, die von etwas ausgehen oder etwas vermuten bzw. in einem Fall sogar von der Vermutung ausgehen. Diese Hypothesen können sich dabei sowohl auf den allgemeinen größeren Zusammenhang beziehen (Beispiele 198 und 200) oder auch konkret Vorhersagen zu den erwarteten Forschungsergebnissen sein (Beispiele 199 und 201).

(198) All else being equal, I expect that as institutional prestige increases, institutions will be better able to realize their objectives, namely, student characteristics. (Grodsky 2007)

(199) I thus hypothesize that the association between black population size and hate crime prosecution is positive until the black population reaches $20 \%$, at which point a negative association emerges. (King 2008)

(200) Ich gehe davon aus, dass die Beteiligung an ehrenamtlichem Engagement in Ländern wie Großbritannien eine höhere Bedeutung als positives Signal an Arbeitgeber hat als in Ländern wie Deutschland, in denen eine zertifizierte berufliche Ausbildung das entscheidende Signal für den Arbeitgeber ist. (Strauss 2009) 
(201) Entsprechend vermute ich, dass die Selbständigen im Vergleich zu den Professionals und den Leitungskräften im schwächeren Maße hochkulturorientiert sind. (Gerhards 2009)

Eine weitere wichtige Funktion, die in diese Kategorie fällt, ist das Auswählen (und Darstellen) theoretischer Grundpositionen. Dies geschieht sehr häufig mit Bezug zum fachlichen Diskurs und innerhalb des Diskurses zu konkreten anderen Arbeiten und/ oder Fachvertretern. Die Autoren dokumentieren hier ihre theoretischen Entscheidungen und Ausrichtungen, die ihrer Analyse zugrunde liegen.

Auch hier benutzen die amerikanischen Autoren eine Vielzahl unterschiedlicher Verben und die Bandbreite reicht vom einfachen und eher passiven Übernehmen (Beispiel 202), Wiederholen und Folgen der Ansichten und Praktiken anderer Wissenschaftler (Beispiel 203) über eine gezielte Mischung und Integration unterschiedlicher Frameworks (Beispiel 204) bis hin zu einer sehr aktiven und bereits wertenden Position, in der der Autor anderen Autoren in ihren Ausführungen zustimmt (Beispiel 205).

(202) As a conceptual guide to capture the Central American immigrants' uncertain legality, I borrow from the work of two anthropologists. (Menjívar 2006)

(203) While some scholars in this tradition emphasize the uniformity and taken-forgrantedness of institutions (e.g., Meyer et al. 1997), I follow those who treat institutions as settlements of conflict among actors with differential power and competing frames (Fligstein 1996; Rao 1998; Roy 1997; Schneiberg and Bartley 2001). (Bartley 2007)

(204) I link tenets of neoinstitutional theory with extant work on criminal punishment to suggest facets of the local legal environment that are conducive to organizational decoupling. (King 2008)

(205) With classic works such as Herberg's Protestant, Catholic, Jew (1955) and Bellah's "Civil Religion in America" (1967), I agree that there is an empirically researchable set of common sacred ideals and norms that has given a basic unity to American culture - at least to the hegemonic culture of the native-born white middle class - since the second half of the 20th century. (Madsen 2009)

Diese Handlungen machen im englischen Korpus 4,85 \% der Forscherhandlungen und 3,48 \% aller ICH-Handlungen aus und sie werden in 18 der Texte verwendet. Im Deutschen dagegen fallen sogar über ein Viertel (27,08\%) aller Forscherhandlungen in diese Kategorie, was auf die gesamte erste Person gerechnet 12,38 \% ausmacht, die sich allerdings nur auf drei Texte verteilen.

\section{Daten erheben}

Mit diesen Handlungen stellt sich der Autor als diejenige Person dar, die die Daten erhebt. Der größte Teil dieser Handlungen beschreibt die konkrete Durchführung der 
Datenerhebung, z. B. das Führen von Interviews (Beispiel 206), das Sichten von Dokumenten oder das Durchführen ethnographischer Studien (Beispiel 207). Diese werden mit Verben wie interview, ask, observe, examine oder conduct (+ Methode) realisiert. Häufig wird hier auch das Verb use mit data oder den konkreten Daten, die in der betreffenden Untersuchung benutzt wurden, kombiniert (Beispiel 208).

Darüber hinaus werden aber auch weiterführende Handlungen, die die Datenerhebung betreffen, thematisiert. Dies reicht von der Entscheidung über die Methode (Beispiel 209) über die Eingrenzung des Datenmaterials (Beispiel 210) bis hin zur Operationalisierung (Beispiel 211).

(206) I interviewed 57 key informants (in 54 separate interviews), with roughly half of the interviews devoted to each of the two cases. (Bartley 2007)

(207) Not surprisingly, Salvadorans and Guatemalans in all the sites where I have conducted fieldwork brought up the importance of the church in their lives. (Menjívar 2006)

(208) I use data from the 1968-2006 March Current Population Surveys (CPS) to examine trends in earnings inequality. (Schwartz 2010)

(209) In order to determine the catalyst of the relationship among these three unions and to evaluate the relationship's nature and quality, I employed a qualitative approach that included in-depth interviews, ethnographic fieldwork, and the analysis of a variety of secondary sources and archival materials. (Kay 2005)

(210) I restrict the institutional samples to community colleges, junior colleges, and comprehensive public and private not-for-profit colleges and universities. (Grodsky 2007)

(211) To avoid these problems, I specifically operationalize Republican control as a time-varying, binary variable indicating whether Republicans in a given stateyear held control over a "veto point" in the legislative process-that is, a point at which it was possible for Republicans to block legislation. (Chen 2007)

Weiterhin ist auffällig, dass der Fokus auch oft auf dem Prozess der Erhebung liegt und nicht nur das Endprodukt dargestellt wird. Dieser Prozess und die mit ihm verbundenen Entscheidungen werden gelegentlich sehr kleinschrittig beschrieben, teilweise werden sogar Schwierigkeiten und andere eher weniger zentrale Handlungen thematisiert, die zwar zu einer Erhebung dazu gehören, die aber dennoch, zumindest aus deutscher Perspektive, zum Teil unerwartet und unnötig wirken.

(212) I was also given access to FAT archives. (Kay 2005)

(213) From the roster of participants of all Mexico-U.S. Dialogos meetings, I compiled a list of national industrial unions that had transnational relationships prior to 1989. (Kay 2005) 
(214) I also have read through the 264 interview transcripts from the first wave. (Vaisey 2009)

(215) I put dates on each reported biographical event by employing my knowledge of local history and other events in the respondents' lives. (Viterna 2006)

(216) Toward this end, I secured access at five plants that were owned and managed by two firms representing distinct tiers within the industry. (Vallas 2006)

Obwohl diese Handlungen im englischen Korpus mit 19,90 \% die zweitgrößte Kategorie der Forscherhandlungen stellen, 14,30 \% der gesamten Handlungen in der ersten Person Singular ausmachen und in 22 der hier untersuchten 24 Texten auftritt, gibt es nicht ein Beispiel aus dem deutschen Korpus, das hierunter fallen würde. Dies ist einer der deutlichsten Unterschiede zwischen den beiden hier untersuchten Korpora und dieses Ergebnis ist konsistent mit Sandersons Beobachtung, dass auch in ihren Texten in Passagen, in denen die Methodologie beschrieben wird, im Englischen häufig die erste Person Singular benutzt werde, im Deutschen jedoch nicht (Sanderson 2008a 139).

Wie oben schon beschrieben, ist die Datenerhebung, gerade bei qualitativen Methoden häufig eine Forschungsphase, in die der Forscher sehr stark als Person involviert ist (s. auch Sanderson 2008a 140). Daher ist eine persönliche Darstellungsweise relativ natürlich und erwartbar. Sanderson hat dagegen beobachtet, dass deutsche Autoren auch in diesen Zusammenhängen eher die dritte Person, teilweise mit unpersönlichen Subjekten, oder auch das Passiv benutzten, was sie als „relatively awkward type of oblique self-reference“ und im Effekt „,somewhat odd“ empfindet (Sanderson|2008a: 139). Ein unsystematischer Querschnitt durch das deutsche Korpus zeigt ebenfalls, dass die Datenerhebung zwar selbstverständlich thematisiert wird, die Autoren dies aber sehr unpersönlich und distanziert darstellen:

(217) In Anlehnung an die Überlegungen von Ajzen und Fishbein (1980) und Friedrichs et al. (1993) wurden die Handlungskonsequenzen in einem schriftlichen Pretest und einer Reihe von qualitativen Interviews und Gruppendiskussionen empirisch ermittelt. (Best 2008)

(218) Die retrospektiven Interviews mit der ostdeutschen Kohorte wurden größtenteils telefonisch von Mai 1996 bis Januar 1998 durchgeführt, während die überwiegend telefonischen Interviews mit der westdeutschen Kohorte etwas später stattfanden (Juni 1998 bis Februar 1999) und über die Lebensverläufe dieser Befragten daher durchschnittlich zwei Jahre länger berichtet werden kann. (Trappe 2006)

(219) Die explorative empirische Analyse beruht auf einer schriftlichen Umfrage, die 2002 in einem sozial heterogenen Leipziger Stadtteil durchgeführt wurde. (Rössel 2006) 


\section{Daten aufbereiten und analysieren}

Hier stellt der Autor die Handlungen dar, die er im Rahmen der Datenanalyse durchgeführt hat. Dazu gehören auch diejenigen Handlungen, mit denen er die Daten für diese Analyse vorbereitet hat. Die benutzten Verben sind beispielsweise examine, code, calculate, measure, estimate, control, analyze in den englischen Texten, daneben kommt auch häufig einfach use bzw. use for in Kombination mit der jeweiligen Methode, dem Wert o. ä. vor. Ähnlich wird im Deutschen vor allem geprüft oder es werden z. B. Variablen verwendet.

(220) To get an idea of just how small a slice this restriction produces, I calculated the falloff from a representative sample after applying an MTO "adjustment" to the Project on Human Development in Chicago Neighborhoods (PHDCN). (Sampson 2008)

(221) I used the sample mean (.75) to replace missing data for five states. (Chen 2007)

(222) In den multivariaten Analysen verwende ich eine binäre Variable, die erfasst, ob die befragte Person in einer der genannten Organisationen aktiv ist. (Strauss 2009)

(223) In einem ersten Schritt habe ich mit Hilfe einer Korrelations- und Faktorenanalyse geprüft, ob es zwischen den verschiedenen Aktivitäten einen korrelativen Zusammenhang gibt. (Gerhards 2009)

Wie auch bei der Datenerhebung werden hier im Englischen teilweise unerwartete und für das deutsche Empfinden sogar merkwürdige Formulierungen benutzt, wie z. B. das „Durchkämmen“ von Interviews.

(224) I also combed through the interviews with an eye toward investigating the conceptions of control in the field. (Quinn 2008)

Auch die Handlungen der Datenaufbereitung und -analyse werden deutlich häufiger von den amerikanischen Autoren als ICH ausgeführt als von den deutschen. Mit $35,84 \%$ der Forscherhandlungen (und 25,76 \% aller ICH-Vorkommen) ist diese Kategorie die größte unter den Forscherhandlungen im englischsprachigen Korpus und sie kommt in 21 Texten vor. Im deutschen Korpus fallen 18,75 \% der Forscher-ICHs sowie $8,57 \%$ aller ICHs in diese Kategorie, was sie zur dritthäufigsten Forscherhandlung im deutschen Korpus macht, die allerdings nur in zwei Texten auftritt.

\section{Ergebnisse erzielen}

Diese Kategorie umfasst alle Handlungen, mit denen der Autor seine Forschungsergebnisse präsentiert und gilt nach Hyland (2002a: 1104) als besonders riskante Rolle. Die englischen Autoren verwenden hier vor allem das Verb find (29 Mal, Beispiele 225 bis 227), daneben kommt auch das Verb identify mehrmals vor (Beispiel 228). Im deutschen Korpus wurden auch für diese Kategorie keine Beispiele identifiziert. 
(225) Although one prominent vein of research emphasizes the overwhelming significance of public opinion in models of policy innovation (Wright et al. 1985; Erikson et al. 1989; Burstein and Linton 2002), I find statistical evidence that party control mattered quite independently of public opinion. (Chen 2007)

(226) Finally, whereas previous studies propose one "typical" route to activism, I find that the variable interaction of networks, biographies, and situational contexts created three distinct paths to guerrilla participation for rural Salvadoran women. (Viterna 2006)

(227) Specifically, I find that, in a typical observation year, supermarket establishments experiencing an initial lawsuit filing increase women's managerial representation by approximately 1.2 times during the filing year more than those not involved in sex discrimination litigation. (Skaggs 2008)

(228) I therefore identify a relationship between the development of a transnational power structure and the emergence of transnational social movements. (Kay 2005)

Mit Blick auf die Forscherhandlungen macht das Auswerten, Interpretieren, Ergebnisse erzielen 4,59\% der englischen ICH-Handlungen aus, insgesamt liegt der Anteil bei $3,30 \%$. Diese Handlung kommt in zehn Texten vor.

\section{Argumentieren für den eigenen Standpunkt}

Unter dieser Kategorie sind alle Handlungen zusammengefasst, mit denen der Autor seine eigene Perspektive, Einstellung und Meinung angibt und in seine Argumentation integriert. Hier vollzieht er also vorrangig seine inhaltliche Positionierung und stellt seinen eigenen Standpunkt dar, häufig mit Bezug zu oder Kritik an anderen Wissenschaftlern, Meinungen, Studien etc.

Entsprechend werden hier Verben wie argue, suggest und assume benutzt, die eine Meinung ausdrücken. Daneben sind hier auch besonders solche Verben und Handlungen relevant, die eine Präferenz für oder die Ablehnung von etwas (Meinungen, Ansichten, Standpunkten etc.) ausdrücken, wie z. B. prefer, (eine Hypothese) zurückweisen, (mit etwas oder jemandem) übereinstimmen etc.

(229) Although this sort of market-based approach is quite prominent in the existing literature on private regulation (e.g., Potoski and Prakash 2005; Spar and Yoffie 2000), I argue that another approach must also be considered. (Bartley 2007)

(230) I suggest that the general absence of law from framing research (and from social movement theory more broadly) limits conceptual and theoretical understanding on how-and with what consequences-challenger groups socially construct their grievances, identities, and objectives (Pedriana 2006) 
(231) Neighborhood effects (and Wilson) are simultaneously broader and more specific than that, and I would not consider spatial job mismatch to be one of the more compelling neighborhood theories. (Sampson 2008)

(232) Dennoch möchte ich die Hypothese, dass die gesamtgesellschaftliche Erfahrung der Arbeitslosigkeit auch die Familiengründungsentscheidungen von Personen betrifft, die selbst nicht mit dem Problem konfrontiert sind, zurückweisen, da die entsprechenden Effekte zu schwach sind. (Brose 2008)

Das Argumentieren für den eigenen Standpunkt ist eine Handlung, die ein deutliches Risikopotenzial birgt, da der Autor hier seine Leser davon überzeugen muss, dass seine Interpretationen und Schlussfolgerungen valide und nachvollziehbar sind. Dabei besteht immer die Gefahr, dass ihm die Leser aufgrund anderer wissenschaftlicher Überzeugungen und Ausrichtungen nicht zustimmen. Dies muss er antizipieren, um Gegenargumente vorwegnehmen und entkräften zu können. Hier ist es für den Autor also besonders wichtig, kompetent und glaubwürdig in seinem Text aufzutreten. Von den amerikanischen Autoren verwenden 21 diese Handlung und sie macht 15,69 \% ihrer Forscherhandlungen und 11,27 \% aller ICH-Vorkommen aus. In den deutschen Texten entspricht diese Handlung noch 12,50 \% der Forscherhandlungen und 5,71 \% aller Handlungen in der ersten Person Singular und sie wird von drei Autoren verwendet.

\section{Aufstellen neuer Claims und neuer Ideen}

Da ein wesentliches Merkmal wissenschaftlicher Artikel ist, dass sie der Community neues Wissen und neue Erkenntnisse bereitstellen, ist es natürlich auch notwendig, diese Neuerungen hervorzuheben. Dies wird in den amerikanischen Texten auch mit Verben und Handlungen vollzogen, die mit der ersten Person Singular kombiniert werden. Dazu gehören vor allem Verben wie develop, extend und add, mit denen sich die Autoren gezielt und aktiv als die "Originatoren" (Tang und John 1999: 29) neuer Ansätze, neuer Entwicklungen und damit neuen Wissens darstellen. Darüber hinaus werden hier auch allgemeine Handlungen mit Verweisen auf die Innovation im Kotext verbunden, wie in Beispiel 236.

(233) I develop a legal framing perspective through historical-narrative analysis of the women's movement in the 1960s. (Pedriana 2006)

(234) In addition, while the institutional literature in economic sociology often focuses on conflicts among different sets of firms (e.g., Fligstein 1996), I extend this model beyond firms and conflicts within markets to a wider array of actors (e.g., NGOs, states, social movements, etc.) engaged in conflicts about the relationship between markets and broader social problems. (Bartley 2007)

(235) I add to existing explanations the critical role of refugee camps as organizational sites of mobilization for many women guerrillas. (Viterna 2006) 
(236) What is new to this analysis is that I decompose the sources of these impacts and show how the changing association has contributed to inequality in different ways in different portions of the earnings distribution. (Schwartz 2010)

In diese Kategorie fällt auch die Begriffsbildung, d. h. die Handlung, mit der neue Begriffe gebildet und in den Diskurs eingeführt werden und die entsprechend mit Verben wie call oder bezeichnen umgesetzt wird.

(237) I simply call this form of the common American sacred the "American religion."(Madsen 2009)

(238) Diese Art und Weise der Handlungszuschreibung bezeichne ich als den Modus der Zuschreibung auf Gründe (vgl. Schulz-Schaeffer 2007: 11 ff., 261 ff.). (Schulz-Schaeffer 2009)

Diese Handlung wird hier unterschieden von der Verfasserhandlung der Terminologieklärung, wo es vorrangig darum geht, den eigenen Gebrauch von Begriffen im bereits bestehenden Diskurs einzuordnen, die im konkreten Text benutzten Definitionen zu erläutern etc. Demgegenüber ist die Begriffsbildung eindeutig eine Forscherhandlung, die neues Wissen und neue Konzepte generiert und damit ein hohes Risiko birgt.

Dass die Begriffe tatsächlich neu sind und erst in den Diskurs integriert werden sollen, zeigt sich beispielsweise daran, dass einige Autoren sie mehrfach in ihrem Text erwähnen (s. Beispiel 239, 242 sowie 243 und 244). Ziel ist es, dass diese Begriffe und neuen Konzepte von der Community akzeptiert und idealerweise auch übernommen werden sollen.

(239) Collectively these practices make up the political genre I will call consentful contention. (Straughn 2005, Absatz 3)

(240) In particular, I attempt to illuminate an important, but recently neglected, phenomenon I will call "consentful contention" using the GDR as a case study. (Straughn 2005, Absatz 9)

(241) In state socialist East Germany, ideological orthodoxy combined with political repression to help cultivate a genre of political interaction I will call consentful contention. (Straughn 2005, Absatz 15)

(242) Fitzpatrick's (1996) valuable work on public letters to the authorities in Stalinist Russia uncovers a rich trove of practices employing what I am calling consentful contention, such as appeals for help and critical opinion letters that present the author as pitiable supplicant, dutiful citizen, and the like, and invoke ideals and objectives of socialism using the language of the state. (Straughn 2005, Absatz 182)

(243) I find that Salvadoran women followed three distinct paths to guerrilla activism: I call them politicized guerrillas, reluctant guerrillas, and recruited guerrillas. (Viterna 2006, Absatz 28) 
(244) Through analysis of my respondents' narratives, I found three clear mobilization patterns, which I have labeled politicized guerrillas, reluctant guerrillas, and recruited guerrillas. (Viterna 2006, Absatz 46)

Als Originator fungieren im englischen Korpus 4,59\% der Forscherhandlungen und 3,30 \% aller ICH-Handlungen in 15 Texten. Im deutschen Korpus sind es 8,33 \% der Forscherhandlungen gegenüber 3,81 \% aller ICHs, die sich allerdings auf nur drei Texte verteilen. Dennoch zeigt der LogLikelihood-Test, dass die englischsprachigen Autoren diese Forscherhandlung offenbar häufiger ausführen $(p<0,001)$.

\section{Danksagen}

Im Rahmen von Danksagungen werden natürlich vor allem Handlungen benutzt, die dem Wortfeld „danken“ zuzurechnen sind, z. B. jemandem danken, sich bedanken, jemandem etwas verdanken, jemandem seinen Dank aussprechen etc. Im Englischen wird das entsprechend mit I thank, I wish to thank, I would like to thank etc. ausgedrückt. Dabei geht der Dank in erster Linie an Kollegen, aber häufig auch an die anonymen Gutachter sowie an Personen und Institutionen, mit oder bei denen die Forschung durchgeführt wurde etc.

(245) For helpful suggestions, I thank Lynne Gerber, Ariel Gilbert-Knight, Heather Haveman, Jerome Karabel, Kristen Luker, Raka Ray, fellow seminar participants, and members of the Center for Culture, Organization, and Politics at the University of California, Berkeley. (Gibson 2005)

(246) I thank an anonymous reviewer for pointing this out. (Quinn 2008)

(247) I also thank the Chicago Police Department, the Chicago Commission on Human Relations, and the Project on Human Development in Chicago Neighborhoods for sharing data. (Lyons 2007)

(248) Ich bedanke mich ganz herzlich bei Anke Offerhaus, die die Daten zusammengetragen hat. (Gerhards 2009)

(249) Für Anregungen und Kritik danke ich Oliver Arránz-Becker, Ulrich Kohler, Johannes Kopp, Anja Steinbach und zwei anonymen Gutachtern. (Lois 2008)

(250) I owe the "cold winds" phrasing to an anonymous reviewer. (Schrank 2010)

(251) I cannot do justice to her generosity and graciousness here. (Vaisey 2009)

(252) Sehr hilfreiche Kommentare habe ich von (...), den Herausgebern dieser Zeitschrift und zwei Gutachtern erhalten. (Gerhards 2009)

(253) Bei der Ausarbeitung dieses Artikels erhielt ich wertvolle Anregungen von den anonymen Gutachtern der KZFSS. (Kern 2007)

Danksagungen kommen in allen englischen Texten vor und werden in 21 Texten auch mit der ersten Person Singular realisiert. Dies kann möglicherweise daran liegen, dass 
das American Journal of Sociology auf seiner Website die Vorgabe macht, dass die erste Fußnote ein Acknolegement Note sein soll ${ }^{18}$

Die deutschen Autoren danken dagegen deutlich weniger und es kommen nur in acht Texten Danksagungen mit einem ICH als Subjekt vor. Prozentual betrachtet machen die Danksagungen aber in den deutschen Texten mit 11 Beispielen insgesamt $22,92 \%$ der Forscherhandlungen aus, was 10,48\% aller Handlungen entspricht, die mit dem ICH realisiert werden. Im Englischen entsprechen die 41 Beispiele dagegen nur 5,23 \% der Forscherhandlungen und 3,76 \% aller Handlungen in der ersten Person Singular.

\section{Andere Forscherhandlungen}

In dieser Kategorie werden alle Forscherhandlungen zusammengefasst, die nicht in eine der oben genannten Kategorien fallen. Mit diesen Handlungen stellt sich der Autor eindeutig als Wissenschaftler dar, d. h. er schreibt sich textexterne Handlungen zu, die er als Mitglied der Scientific Community oder im engeren Sinne als jemand, der an einer wissenschaftlichen Institution tätig ist, ausführt. Konkret stellen sich die Autoren der hier untersuchten Texte beispielsweise als Autoren anderer Texte, als Teilnehmer an wissenschaftlichen Konferenzen oder als Antragsteller dar.

(254) I prepared this article while I was a visiting scholar at the Maison des Sciences de l'Homme, Paris, in spring 2003 and would like to acknowledge the institutional support. (Menjívar 2006)

(255) I presented it in the thematic session Culture, Migration and Diaspora at the American Sociological Association meetings in Atlanta, August 2003, organized by (...). (Menjívar 2006)

(256) I presented myself as an independent but sympathetic researcher who wanted to understand the changes impinging on industrial work today. (Vallas 2006)

(257) Im internationalen EU-Forschungsprojekt „Socio-economic models of a knowledge-based society" habe ich mit (...) (Projektleiter) und (...) zusammengearbeitet, denen ich hier an erster Stelle meinen Dank ausspreche (...). (Teipe 2009)

(258) Diese begriffliche Präzisierung sowie viele andere relevante Einsichten und Ideen verdanke ich (...), mit dem ich im Frühjahr 2005 ein Forschungsprojekt unter dem Titel „Selig- und Heiligsprechungsverfahren in der katholischen Kirche. Die Repersonalisierung des Charisma im Rahmen seiner Versachlichung" konzipiert und bei der DFG eingereicht habe. (Bienfait 2006)

Diese anderen Forscherhandlungen machen im Englischen Korpus 2,55 \% und 1,83 \% aller Handlungen in der ersten Person Singular aus und kommen in zehn Texten vor. Im deutschen Korpus sind es 8,33\% bzw. 3,81 \% in drei Texten.

\footnotetext{
${ }^{18}$ s. http://www.press.uchicago.edu/journals/ajs/instruct.html?journal=ajs, letzter Zugriff: 13.9.13.
} 


\subsection{Andere}

\begin{tabular}{|l|l|l|l|}
\hline Rolle & Englisch absolut & Deutsch absolut & gesamt \\
\hline Andere & 12 & 10 & 22 \\
\hline
\end{tabular}

Tabelle 5.25: Übersicht Andere absolut

Die wichtigste Rollenfunktion, die das ICH neben dem Verfasser und dem Forscher ausführen kann, ist die des „Repräsentanten“ einer Gruppe oder sogar der gesamten Menschheit. Dies ist vergleichbar mit dem Gebrauch von WIR und MAN als Gemeinschafts-WIR bzw. -MAN (s. Kap. 3.3.1.2 bzw. 3.3.1.3) und diese Gebrauchsform wurde in vorhergehenden Untersuchungen als eindeutig studentisch gewertet (vgl. z. B. Tang und John 1999: Steinhoff 2007), eine Einschätzung, die aufgrund dieser Beispiele hinterfragt oder zumindest differenziert werden sollte. In den deutschen Texten wurde das ICH zehmmal in dieser Form benutzt, was 9,52\% aller ICH-Vorkommen ausmacht, in den englischen kam es $12 \mathrm{Mal}(1,1 \%)$ vor.

(259) No one has the option of speaking to me after I speak to the group (the P-tie Aj0-XiA), for instance, if I never speak to the group (the initial Aj0). (Gibson 2005)

(260) I might use a voucher to move to a lower-poverty neighborhood than where a control group member lives, for example, but that neighborhood may be on a downward trajectory (e.g., with declining house values) while the control neighborhood is stably poor. (Sampson 2008)

(261) Liebe und Vertrauen münden so in einer „Konsensfiktion über Gemeinsamkeiten" (Hahn 1983: 222) - wenn der andere so ist wie ich, dann gibt es keinen Grund, anzunehmen, er könne nicht optimal zu mir passen oder gar opportunistische Neigungen haben. (Schnabel 2005)

(262) Wenn mir beispielsweise ein Tischnachbar den Brotkorb hinhält, werde ich normalerweise nicht extra nachfragen, ob er damit die Absicht verfolgt, mir das Brot zu reichen. (Schulz-Schaeffer 2009)

(263) So kann ich in gewissen Abständen indisch essen gehen, arabische Musik hören, Filme aus einem anderen Kulturkreis anschauen oder meine Wohnung mit fremd-kulturellen Accessoires ausstatten, ohne die Grundlage des durchschnittlichen westeuropäischen Wertmusters zu verlassen. (Schwinn 2006)

\subsubsection{Vergleich 1. Person Plural}

Äquivalent zur ersten Person Singular werden hier die Vorkommen der ersten Person Plural im englischen und deutschen Korpus verglichen. Insgesamt haben die Autoren der hier untersuchten Texte $320 \mathrm{Mal}$ das WIR bzw. WE im Nominativ als Subjekt 
bzw. Agens benutzt (285 Mal WE und 35 Mal WIR) und damit vorwiegend Forscherhandlungen ausgeführt. Auf Verfasserhandlungen entfallen nur insgesamt 28 der 320 Vorkommen (s. Tab. 5.26.

Aus diesem Grund werden die Verfasserhandlungen genau wie andere Handlungen, die weder in die Forscher- noch die Verfasserkategorie fallen, im Folgenden gesammelt behandelt. Nur die Forscherhandlungen werden wie bei der Betrachtung der ersten Person Singular weiter differenziert.

\begin{tabular}{|l|l|l|l|l|}
\hline Rolle & $\begin{array}{l}\text { Englisch } \\
\text { absolut }\end{array}$ & $\begin{array}{l}\text { Deutsch abso- } \\
\text { lut }\end{array}$ & gesamt & $\mathbf{G}^{2}$ \\
\hline $\begin{array}{l}\text { Verfasser } \\
\text { gesamt }\end{array}$ & 16 & 12 & 28 & 1,11 \\
\hline Andere & 36 & 1 & 37 & 22,13 \\
\hline $\begin{array}{l}\text { Forscher } \\
\text { gesamt }\end{array}$ & 233 & 22 & 255 & 87,06 \\
\hline \hline $\begin{array}{l}\text { Fokussieren } \\
\text { der For- } \\
\text { schungsfrage }\end{array}$ & 12 & 0 & 12 & 9,71 \\
\hline $\begin{array}{l}\text { Hypothesen } \\
\text { und Grundan- } \\
\text { nahmen }\end{array}$ & 43 & 7 & 50 & 9,7 \\
\hline $\begin{array}{l}\text { Daten erheben } \\
\text { /analysieren }\end{array}$ & 76 & 9 & 85 & 23,86 \\
\hline $\begin{array}{l}\text { Ergebnisse er- } \\
\text { zielen }\end{array}$ & 18 & 1 & 19 & 8,93 \\
\hline $\begin{array}{l}\text { Argumenta- } \\
\text { tion/Arguer }\end{array}$ & 5 & 2 & 7 & 0,07 \\
\hline Begriffe bilden & 6 & 0 & 6 & 9,85 \\
\hline $\begin{array}{l}\text { Bezug auf } \\
\text { Community- } \\
\text { Wissen }\end{array}$ & 28 & 0 & 31 & 9,54 \\
\hline $\begin{array}{l}\text { Appell an } \\
\text { Community }\end{array}$ & 32 & 13 & 13 & 10,52 \\
\hline unklar & 0 & 69 & \\
\hline
\end{tabular}

Tabelle 5.26: Übersicht Rollen in der 1. Person Plural absolut 


\begin{tabular}{|l|l|l|}
\hline Rolle & Englisch & Deutsch \\
\hline Verfasser gesamt & 5,61 & 34,29 \\
\hline Andere & 12,63 & 2,86 \\
\hline Forscher gesamt & 81,75 & 62,86 \\
\hline $\begin{array}{l}\text { Fokussierung der For- } \\
\text { schungsfrage }\end{array}$ & 4,21 & 0 \\
\hline $\begin{array}{l}\text { Hypothesen und Grund- } \\
\text { annahmen }\end{array}$ & 15,90 & 20,00 \\
\hline $\begin{array}{l}\text { Methode/Daten- } \\
\text { erhebung/-analyse }\end{array}$ & 26,67 & 25,21 \\
\hline Ergebnisse & 6,32 & 2,86 \\
\hline Argumentation/ Arguer & 1,75 & 5,71 \\
\hline Begriffsbildung & 2,11 & 0 \\
\hline $\begin{array}{l}\text { Bezug auf Community- } \\
\text { Wissen }\end{array}$ & 9,82 & 8,57 \\
\hline Appell an Community & 11,23 & 0 \\
\hline unklar & 4,56 & 0 \\
\hline
\end{tabular}

Tabelle 5.27: prozentuale Verteilung der Rollen in der 1. Person Plural

Bis auf wenige Ausnahmen werden die WEs in den englischen Texten häufiger in den jeweiligen Funktionen benutzt als die deutschen. Für die Kategorien Appell an Community und Methode/Datenerhebung/Analyse sowie beim Vergleich der Forscherhandlungen insgesamt und der anderen Handlungen konnte ein Signifikanzwert von $p<0,0001$ nachgewiesen werden. Für die Verfasserhandlungen sowie die Argumentation wurde kein nennenswerter Unterschied festgestellt. Die restlichen Handlungen werden mit einem Wert von $\mathrm{p}<0,01$ häufiger von den englischsprachigen Autoren benutzt.

Bei der Verteilung auf die einzelnen Texte (s. Tabelle 5.28) fällt auf, dass nicht alle Autoren ein WIR bzw. WE benutzt haben, dies betrifft im Englischen fünf und im deutschen zehn Texte. Dabei haben die amerikanischen Autoren allerdings, wenn sie das WE benutzt haben, auf jeden Fall auch Forscherhandlungen damit durchgeführt, während von den deutschen Autoren nur in zehn der insgesamt 15 Texte, in denen das WIR mit Referenz auf den Autor vorkam, dieses WIR auch mit Forscherhandlungen kombiniert wurde. Diese zehn Autoren verwendeten das WIR auch nur in Kombination mit fünf der insgesamt neun im gesamten Korpus gefundenen Handlungskategorien, wohingegen die amerikanischen Autoren alle neun Handlungen mit dem WE ausführten. Dabei nahmen mit 14 Autoren die meisten Bezug auf das Community-Wissen.

Beim Blick auf die prozentuale Verteilung der Rollen auf die gesamte Zahl der ersten Person Plural fällt auf, dass die deutschen Autoren prozentual betrachtet deutlich 


\begin{tabular}{|l|l|l|l|}
\hline Rolle & Englisch & Deutsch & gesamt \\
\hline 1. Pers. PL gesamt & 20 & 15 & 35 \\
\hline Verfasser gesamt & 6 & 9 & 15 \\
\hline Andere & 7 & 1 & 8 \\
\hline Forscher gesamt & 20 & 10 & 30 \\
\hline \hline $\begin{array}{l}\text { Fokussierung der } \\
\text { Forschungsfrage }\end{array}$ & 5 & 0 & 5 \\
\hline $\begin{array}{l}\text { Hypothesen und } \\
\text { Grundannahmen }\end{array}$ & 12 & 4 & 16 \\
\hline $\begin{array}{l}\text { Methode/Da- } \\
\text { tenerhebung/ } \\
\text {-analyse }\end{array}$ & 11 & 4 & 15 \\
\hline Ergebnisse & 6 & 1 & 7 \\
\hline $\begin{array}{l}\text { Argumentation/ } \\
\text { Arguer }\end{array}$ & 4 & 2 & 6 \\
\hline Begriffsbildung & 3 & 0 & 3 \\
\hline $\begin{array}{l}\text { Bezug auf Com- } \\
\text { munity-Wissen }\end{array}$ & 14 & 2 & 16 \\
\hline $\begin{array}{l}\text { Appell an Com- } \\
\text { munity }\end{array}$ & 10 & 0 & 10 \\
\hline unklar & 3 & 0 & 3 \\
\hline
\end{tabular}

Tabelle 5.28: Verteilung der 1. Person Plural auf Texte

öfter das WIR für Verfasserhandlungen benutzen als ihre amerikanischen Kollegen. In den deutschen Texten sind dies 35,29\% gegenüber 5,61 \% in den englischen, wobei sich für diese Verteilung jedoch noch kein p-Wert $<0,05$ herausgestellt hat (s. Tabelle 5.26).

Deutlicher ist demgegenüber der Unterschied bei den Forscherhandlungen, bei denen 81,75\% der englischen WE 62,86 \% der deutschen WIR gegenüberstehen. Die erste Person Plural wird also genau wie die erste Person Singular in den englischen Texten häufiger in Kombination mit Forscherhandlungen als mit Verfasserhandlungen benutzt. Auch im Deutschen ist diese Tendenz für die Pluralpronomen deutlich ersichtlich ${ }^{19}$ Die folgenden Tabellen 5.29 bis 5.31 zeigen die Verteilung der Pluralpronomen nach Referenz.

\footnotetext{
${ }^{19} \mathrm{Im}$ Singular besteht im Deutschen jedoch eine leichte Tendenz zum Gebrauch der Verfasserhandlungen (s. Kap. 5.2.2.1.
} 


\begin{tabular}{|c|c|c|c|c|}
\hline & \multicolumn{2}{|c|}{ Autor } & \multicolumn{2}{|c|}{ Autor + Andere } \\
\hline Rolle & E & D & E & D \\
\hline $\begin{array}{l}\text { Verfasser } \\
\text { gesamt }\end{array}$ & 0 & 2 & 0 & 0 \\
\hline Andere & 0 & 0 & 14 & 0 \\
\hline $\begin{array}{l}\text { Forscher } \\
\text { gesamt }\end{array}$ & 47 & 11 & 28 & 0 \\
\hline $\begin{array}{l}\text { Fokussierung } \\
\text { der } \quad \text { For- } \\
\text { schungsfrage }\end{array}$ & 5 & 0 & 1 & 0 \\
\hline $\begin{array}{l}\text { Hypothesen } \\
\text { und Grundan- } \\
\text { nahmen }\end{array}$ & 8 & 1 & 0 & 0 \\
\hline $\begin{array}{l}\text { Methode/Da- } \\
\text { tenerhebung/ } \\
\text {-analyse }\end{array}$ & 21 & 8 & 27 & 0 \\
\hline Ergebnisse & 9 & 1 & 0 & 0 \\
\hline $\begin{array}{l}\text { Argumenta- } \\
\text { tion/ Arguer }\end{array}$ & 3 & 1 & 0 & 0 \\
\hline Begriffsbildung & 1 & 0 & 0 & 0 \\
\hline $\begin{array}{l}\text { Bezug auf } \\
\text { Community- } \\
\text { Wissen }\end{array}$ & 0 & 0 & 0 & 0 \\
\hline $\begin{array}{l}\text { Appell an } \\
\text { Community }\end{array}$ & 0 & 0 & 0 & 0 \\
\hline unklar & 0 & 0 & 0 & 0 \\
\hline
\end{tabular}

Tabelle 5.29: Verteilung nach Referenz: exklusiv 


\begin{tabular}{|c|c|c|c|c|}
\hline \multirow[b]{2}{*}{ Rolle } & \multicolumn{2}{|c|}{ Pseudo-inklusiv } & \multicolumn{2}{|c|}{ Teamwork-WIR } \\
\hline & $E$ & D & $\mathrm{E}$ & D \\
\hline $\begin{array}{l}\text { Verfasser } \\
\text { gesamt }\end{array}$ & 1 & 0 & 14 & 10 \\
\hline Andere & 0 & 0 & 0 & 0 \\
\hline $\begin{array}{l}\text { Forscher } \\
\text { gesamt }\end{array}$ & 33 & 1 & 24 & 3 \\
\hline $\begin{array}{l}\text { Fokussierung } \\
\text { der } \quad \text { For- } \\
\text { schungsfrage }\end{array}$ & 3 & 0 & 3 & 0 \\
\hline $\begin{array}{l}\text { Hypothesen } \\
\text { und Grundan- } \\
\text { nahmen }\end{array}$ & 14 & 1 & 8 & 1 \\
\hline $\begin{array}{l}\text { Methode/Da- } \\
\text { tenerhebung/ } \\
\text {-analyse }\end{array}$ & 14 & 0 & 4 & 1 \\
\hline Ergebnisse & 1 & 0 & 5 & 0 \\
\hline $\begin{array}{l}\text { Argumenta- } \\
\text { tion/ Arguer }\end{array}$ & 0 & 0 & 2 & 0 \\
\hline Begriffsbildung & 1 & 0 & 1 & 0 \\
\hline $\begin{array}{l}\text { Bezug auf } \\
\text { Community- } \\
\text { Wissen }\end{array}$ & 0 & 0 & 0 & 1 \\
\hline $\begin{array}{l}\text { Appell an } \\
\text { Community }\end{array}$ & 0 & 0 & 0 & 0 \\
\hline unklar & 0 & 0 & 1 & 0 \\
\hline
\end{tabular}

Tabelle 5.30: Verteilung nach Referenz: pseudo-inklusiv und Teamwork-WIR 


\begin{tabular}{|c|c|c|c|c|c|c|}
\hline & & W und FK & & eis-WIR & & hafts-WIR \\
\hline Rolle & $\mathbf{E}$ & D & E & D & $E$ & D \\
\hline $\begin{array}{l}\text { Verfasser } \\
\text { gesamt }\end{array}$ & 0 & 0 & 0 & 0 & 1 & 0 \\
\hline Andere & 0 & 0 & 4 & 0 & 18 & 1 \\
\hline $\begin{array}{l}\text { Forscher } \\
\text { gesamt }\end{array}$ & 27 & 3 & 72 & 4 & 2 & 0 \\
\hline $\begin{array}{l}\text { Fokus- } \\
\text { sierung } \\
\text { der For- } \\
\text { schungs- } \\
\text { frage }\end{array}$ & 0 & 0 & 1 & 0 & 0 & 0 \\
\hline $\begin{array}{l}\text { Hypothe- } \\
\text { sen und } \\
\text { Grundan- } \\
\text { nahmen }\end{array}$ & 10 & 1 & 3 & 3 & 0 & 0 \\
\hline $\begin{array}{l}\text { Methode/ } \\
\text { Daten- } \\
\text { erhebung/ } \\
\text {-analyse }\end{array}$ & 6 & 0 & 4 & 0 & 0 & 0 \\
\hline Ergebnisse & 0 & 0 & 1 & 0 & 2 & \\
\hline $\begin{array}{l}\text { Argu- } \\
\text { menta- } \\
\text { tion/ } \\
\text { Arguer }\end{array}$ & 0 & 1 & 0 & 0 & 0 & 0 \\
\hline $\begin{array}{l}\text { Begriffs- } \\
\text { bildung }\end{array}$ & 2 & 0 & 1 & 0 & 0 & 0 \\
\hline $\begin{array}{l}\text { Bezug } \\
\text { auf Com- } \\
\text { munity- } \\
\text { Wissen }\end{array}$ & 0 & 1 & 28 & 1 & 0 & 0 \\
\hline $\begin{array}{l}\text { Appell an } \\
\text { Commu- } \\
\text { nity }\end{array}$ & 9 & 0 & 23 & 0 & 0 & 0 \\
\hline unklar & 0 & 0 & 12 & 0 & 0 & 0 \\
\hline
\end{tabular}

Tabelle 5.31: Verteilung nach Referenz: zwischen Teamwork- und Fachkreis-WIR, Fachkreis-WIR, Gemeinschafts-WIR 


\subsection{Verfasser}

Das Verfasser-WIR wird im Vergleich zum Forscher-WIR deutlich seltener benutzt. In den untersuchten Texten wurden nur insgesamt 28 Beispiele, 16 englische und 12 deutsche, dafür gefunden. Im deutschen Korpus entspricht das 34,29 \% der WIR-Vorkommen, während fast doppelt so viele (62,86\%) Forscherhandlungen erfüllen. Im englischen Korpus ist dieser Unterschied sogar noch deutlicher, da neben den 81,75 \% Forscher-WEs nur 5,61 \% aller WEs im Rahmen von Verfasserhandlungen verwendet werden. Dies ist jedoch nicht weiter verwunderlich, wenn man bedenkt, dass alle hier untersuchten Texte von einem Einzelautor geschrieben wurden und dass entsprechend die wesentlichen Handlungen als Verfasser auch von dieser Einzelperson im Singular durchgeführt werden sollten. Da die Verfasserhandlungen darüber hinaus vorrangig dazu dienen, den Leser durch den Text zu führen und metakommunikativ zu begleiten, ist es ebenfalls wenig überraschend, dass die im Rahmen von Verfasserhandlungen benutzen Pluralpronomen bis auf jeweils wenige Ausnahmen auf den Autor und den Leser, das sog. Teamwork-WIR, referieren.

Die bei Weitem meisten Vorkommen des Verfasser-WIR im Englischen (10 von 16) werden mit dem Verb see kombiniert. Im Gegensatz zum inhaltlich ähnlichen show, das häufig mit ICH kombiniert und zur verbalen und graphischen Darstellung gezählt wird (s. S. 101), wird das Sehen in Kombination mit dem WIR deutlich häufiger zur Textstrukturierung als Vor- oder Rückverweis verwendet. Dies zeigt sich vor allem daran, dass es selten im Präsens, sondern eher in Vergangenheits- oder Zukunftsformen verwendet wird, was bei der ersten Person Singular in Kombination mit show bzw. zeigen so gut wie nicht vorkommt.

(264) As we shall see in more detail below, our interviewees had all at least felt (and sometimes seen) the presence of God and the spirits. (Madsen 2009)

(265) As we will see presently, the consequences would have been even more dire than before. (Straughn 2005)

(266) As we saw, much depends on the credibility of the consentful standpoint as performed by the individual petitioner; thus, there is one component of petitioner capacity, "worthiness,"that may interact with the consentful standpoint of a petition to increase the likelihood of state compliance. (Straughn 2005)

(267) As we have seen, in this particular case at least, neither of these stories can make sense of all of the data. (Vaisey 2009)

(268) Wie wir genauer sehen werden, ist die von Luhmann veranschlagte erste Ausdifferenzierung der Liebe als Kommunikationssystem in einer Oberschicht des 17. Jahrhunderts nur ein Effekt dieses Zusammenspiels. (Becker 2005) 
(269) Die Berufspositionen werden von Bourdieu, wie wir in Kapitel 2 gesehen haben, zur Beschreibung der Klassen und Klassenfraktionen benutzt. (Gerhards 2009)

In Beispiel 264 wird der Leser auf etwas aufmerksam gemacht, das er demnächst lesen wird. Dies geschieht erstens durch die Bildung des Futur mit shall und zweitens durch den direkten lokalen Verweis anhand des below. Außerdem macht dieses Beispiel noch einmal die unterschiedlichen Referenzmöglichkeiten der ersten Person Plural deutlich, da nämlich das WE, um das es hier geht, eine andere Personengruppe bezeichnet, als das OUR, das hier nicht weiter untersucht wird. Das WE bezieht den Leser eindeutig mit ein, während das OUR deutlich als exklusiv zu verstehen ist.

Vergleichbares ist auch in Beispiel $265 \mathrm{zu}$ sehen, wobei hier das will-Futur genutzt wird und mit presently dieses Mal ein temporales Adverb den Vorverweis ausdrückt. In den beiden folgenden Beispielen 266 und 267 erfolgen die Rückverweise nur anhand der Vergangenheitsformen. Entsprechend fungieren auch in den beiden deutschen Beispielen 268 und 269 die Zeitformen des Verbs als Mittel zum Vor- bzw. Rückverweis, in Beispiel 269 wird der Rückverweis dazu noch durch die Angabe Kapitel 2 verstärkt.

Daneben werden aber natürlich auch andere Verben für Vor- und Rückverweise sowie auch andere Handlungen der Textstrukturierung verwendet (s. Beispiele 271 und 273). Insbesondere werden über das WIR häufig direkte Verweise auf Tabellen, Beispiele o. ä. realisiert, wie in den Beispielen 270 und 272.

(270) That they are distant from subordinancy in figure 3 suggests that subordinates avoided these modes of behavior vis-à-vis their superiors, and this is confirmed by table 3, where we find subordinancy negatively associated with all three of these P-ties (though the effect on AjB-BiY is not significant), and with $\mathrm{ABj}-\mathrm{XiY}$ as well. (Gibson 2005)

(271) Fassen wir daher noch einmal kurz zusammen, worin die entscheidenden Unterschiede zwischen Bourdieus und Luhmanns Ansatz der Differenzierung liegt: [...]. (Becker 2005)

(272) Betrachten wir zwei Beispiele, in denen Ego beidesmal das gegen den Willen von Alter gerichtete Ziel hat, dass Alter sterben solle. (Schulz Schaeffer 2009)

(273) Dies hatten wir als eine weitere zu berücksichtigende Konstellation in der Modellskizze unter Abschnitt II hervorgehoben. (Schwinn 2006)

Eine weitere Rolle, die in der Form nicht vom ICH übernommen wurde, ist diejenige, die Tang und John (1999) als ' $I$ ' as the guide through the essay beschrieben haben (s. Kap. 3.3.2.1). Hier führt der Autor den Leser durch den Text, weist ihn auf bestimmte wichtige Dinge hin und verortet ihn in Zeit und Raum, um letztlich am Ziel, d.h. an den vom Autor vertretenen Schlussfolgerungen anzukommen. Das Bild des Guides, 
der den Leser durch den Text wie durch eine Ausstellung oder ein fremdes Land führt, wird in den folgenden Beispielen besonders deutlich.

(274) In between, as we move from left to right, are the classical right-to-rule ideologies (e.g., divine right of kings, traditionalism, prophetic charisma) and what may be called "corrupted" ideologies, resulting in a form of rule that purports legitimacy, but is perceived by most citizens as "cynical". (Straughn 2005)

(275) Schließlich kommen wir zu den Motiven für die Proteste, die in hohem Maße von konkreten Missständen in den Teilsystemen bestimmt waren (s. Tabelle 1). (Kern 2007)

(276) Damit sind wir bei den Schwächen von Lebensstiltypologien angelangt. (Otte 2005)

\subsection{Forscher}

Der Großteil der Pluralpronomen wird mit Handlungen der Forscherrolle kombiniert. $81,75 \%$ der englischen WE und 62,86 \% der deutschen WIR fallen in diese Kategorie. Daher wurde hier, im Gegensatz zur Verfasserrolle, noch weiter differenziert und es werden im Folgenden die einzelnen Handlungen, die das WIR als Subjekt und Agens ausführt, näher untersucht.

\section{Fokussieren der Forschungsfrage}

Hier grenzt das WIR den Forschungsfokus ein, macht deutlich, auf welche Aspekte konkret Wert gelegt wurde und welche Perspektiven bei der Forschung eingenommen wurden (z. B. mit consider, Beispiel 278). Diese Handlung, die äquivalent auch mit der ersten Person Singular gefunden wurde, wird mit dem Plural nur von den amerikanischen Autoren kombiniert (4,21\% aller Pluralpronomen in fünf der 25 Texte), im deutschen Korpus wurden dafür keine Beispiele gefunden. Die Referenzen der WE in dieser Kategorie sind bis auf das Gemeinschafts-WIR praktisch auf alle Referenzkategorien verteilt, wobei mit fünf Beispielen (von insgesamt 12) fast die Hälfte sich nur auf den Autor beziehen. Dies ist nicht weiter verwunderlich, da der Autor natürlich derjenige ist, dessen Forschungsinteresse im betreffenden Artikel wiedergegeben wird.

Die Verben, die für diese Handlung benutzt werden, zeigen dieses Interesse entsprechend deutlich. Neben den eher persönlichen Angaben we are interested in (Beispiel 277) und we want to know (Beispiel 278) werden auch neutralere Angaben wie look at/to oder consider (Beispiel 279) verwendet. In einem Beispiel wurde der Forschungsfokus auch negativ über we limit our analysis to definiert (Beispiel 280)

(277) The two-and-a-half-year lag between waves may be longer than ideal, but since we are interested in durable moral dispositions, a lag of this length should not pose a significant problem. (Vaisey 2009) 
(278) Or more precisely, we want to know whether the observed association is significantly greater (or smaller) than we would have expected given a null model assuming no network effects. (Gibson 2005)

(279) This problem becomes even more complex if we consider that most families living in severely disadvantaged neighborhoods have lived in similar environments for multiple generations (Sharkey 2008), raising the possibility that the influence of disadvantage extends across generations. (Sampson 2008)

(280) Or, to put the matter differently, the rate of firm-level mortality was only $26 \%$ overall—or $31 \%$ (18 out of 58) if we limit our analysis to the more homogeneous textile, clothing, and footwear firms that tend to dominate the zone. (Schrank 2010)

\section{Darstellen von Hypothesen, Ausgangsannahmen, theoretischen Grundpositionen}

Mit diesen Handlungen stellen die Autoren ihre theoretischen Ausgangspositionen und grundlegenden Annahmen und Hypothesen dar. Auch diese Handlungen werden häufiger von amerikanischen Autoren benutzt als von deutschen. Die prozentuale Verteilung auf die Gesamtzahl der ersten Person Plural zeigt, dass 15,90\% der englischen WEs und sogar 20,00 \% der deutschen WIR diese Funktion erfüllen. Allerdings wird auch diese Handlung mit dem WIR nicht von allen Autoren gleichermaßen benutzt, im Englischen kommt sie in 12 der 25 Texte vor, im Deutschen nur in vier.

Dabei ist die Referenz der 43 englischen Vorkommen relativ gleichmäßig verteilt, einzig das Gemeinschafts-WIR wird in dieser Funktion nicht benutzt, was damit zu erklären ist, dass diese Handlungen in erster Linie die wissenschaftliche Community betreffen. Interessant ist sicherlich, dass gerade diese Handlung v. a. im Englischen auch mit dem pseudo-inklusiven WIR verwendet wird (z. B. Beispiel 282), was sicherlich daran liegt, dass gerade diese Referenzform besonders persuasiv wirkt. Die Autoren streben an, ihre Leser von ihren Ausgangspositionen zu überzeugen, da dies die Grundlage dafür ist, dass sie sich auch von der weiteren Argumentation überzeugen lassen. Mit 14 Beispielen ist diese Handlung neben der Darstellung von Methode, Datenerhebung und -analyse (Kap. 5.2.2.2.2) diejenige mit den meisten Vorkommnissen dieser Referenzkategorie im Englischen. Im Deutschen überwiegt mit drei Beispielen dagegen die Referenz auf den Fachkreis (s. Beispiel 283, wo der Autor seine theoretische Annahme über den Bezug zu einem anderen Autor stärkt), außerdem gibt es jeweils ein Vorkommnis mit den Referenzen nur Autor, pseudo-inklusiv und Teamwork und eines, das sich nicht eindeutig zwischen Teamwork und Fachkreis einordnen lässt.

Die Verben, die für diese Handlunggskategorie verwendet werden, sind wenig überraschend. Genau wie die erste Person Singular wird das WIR hier auch vorrangig mit den Verben expect, assume bzw. ausgehen von und annehmen kombiniert. 
(281) Ultimately, these are empirical questions, but before turning to the data we must consider theoretically why we should expect affirmative answers. (Gerber 2006)

(282) Thus, this question essentially generated information about work collaboration/interdependence that was above and beyond the ambient levels of interdependence we would expect in any work group. (Gibson 2005)

(283) Wir finden diese Version im Prinzip schon bei Ernest Nagel (1961: 354), wenn er für die Mikro-Makro-Verbindungen, die bei Lindenberg und Esser dann Transformationsregeln genannt werden, neben solchen analytischen Charakteren solche faktischer oder materialer Art postuliert. (Albert 2005)

(284) Wenn wir annehmen, dass Rationalität Determiniertheit bedeutet, dann gilt: ,... if a person's preferences are incomplete, there are situations in which he will make choices without having reasons for these choices. We might want to say that such choices are, if not exactly irrational, at least non-rational“ (Sugden 1991: 761). (Schnabel 2005)

(285) Wir gehen davon aus, dass Personen, die kleine Kinder haben - gemessen durch die Frage „Anzahl der Kinder unter 10 Jahren“- stärker an den Haushalt gebunden sind und entsprechend seltener an außerhäuslichen kulturellen Aktivitäten teilnehmen können. (Gerhards 2009)

\section{Daten erheben und analysieren}

Die Darstellung vom methodischen Vorgehen, der Datenerhebung und der Auswertung wird hier zusammengefasst, da im Plural deutlich weniger Beispiele vorliegen als für die gleiche Kategorie mit der ersten Person Singular. Auch hier wurde der Großteil der Beispiele wieder in den englischen Texten gefunden (76 Vorkommnisse in 11 Texten gegenüber neun in vier Texten im deutschen Korpus). Betrachtet man die prozentuale Verteilung der Pluralpronomen, die mit dieser Handlung kombiniert werden, zeigt sich fast das gleiche Ergebnis: 26,67 \% der englischen WE und 25,21 \% der deutschen WIR fallen in diese Kategorie.

Bei der Verteilung in die verschiedenen Referenzkategorien ergab sich, dass die bei Weitem meisten Vorkommnisse dieser Handlungen mit einem exklusiven WIR kombiniert wurden. So waren bei 21 der englischen Beispiele nur der Autor und bei 27 der Autor und Andere die bezeichneten Personen. Daneben fielen aber auch noch 14 Beispiele in die Kategorie der pseudo-inklusiven Referenz und ebenfalls insgesamt 14 in die genuin inklusiven Referenzen Teamwork- (vier Vorkommnisse) und Fachkreis-WIR (vier Vorkommnisse) oder sie waren nicht eindeutig in eine dieser beiden Kategorien einzuordnen (zwischen Teamwork und Fachkreis, sechs Vorkommnisse). Und auch bei den deutschen Beispielen war diese Tendenz eindeutig, da acht der neun Vorkommnisse nur auf den Autor referierten und das übrige ein Teamwork-WIR war. 
Dieses Ergebnis kann so erklärt werden, dass die hier beschriebenen Handlungen typischerweise von den Autoren außerhalb der Texterstellung vorgenommen wurden und insofern die Leser (potenzieller) Texte nicht weiter berücksichtigt werden müssen. Diese Handlungen werden hier also in den meisten Fällen einfach referiert. Da aber im Rahmen eines Artikels trotzdem die Notwendigkeit besteht, die Leser auch von dem methodischen Vorgehen im Rahmen der dem Artikel meist vorgelagerten Datenerhebung und -analyse zu überzeugen, ist die gelegentliche Verwendung inklusiver WIRVarianten damit zu erklären, dass sie als rhetorische Mittel genau diesen persuasiven Effekt erzielen sollen20

Auch für diese Kategorie sind die in den untersuchten Texten verwendeten Verben denen, die mit der ersten Person Singular kombiniert wurden, sehr ähnlich. Vorrangig werden die Handlungen wiedergegeben, die im Datenerhebungs- bzw. Auswertungsprozess anfielen, wie z. B. interview, study, estimate, observe, posit etc. Im Deutschen wurden vorrangig Daten erhoben, so dass die Autoren sie dann besitzen und sie in verschiedenen Kategorien zusammengefasst werden können.

(286) Most of the lay members whom we interviewed, however, talked as if their choice was between apparently viable alternatives, although once they made the choice, they realized that it was absolutely right for them. (Madsen 2009)

(287) We can obtain the odds of departure by exponentiating the individual regression coefficients in the dynamic logit model. (Schrank 2010)

(288) Wir haben für die Spielzeiten 2002/2003 und 2003/2004 die Programme der über das Internet zugänglichen Opernhäuser weltweit erhoben. (Gerhards 2009)

(289) Fassen wir noblesse de robe und haute robe zu einer sozialen Gruppe zusammen, dann wird besonders deutlich, dass sowohl die noblesse de robe als auch die noblesse de race eine Bindung mit der Tochter aus dem Robenadel anstrebten (Tabelle 2): (...). (Becker 2005)

\section{Ergebnisse erzielen}

Die Ergebnisse werden im Englischen in 18 Fällen mit dem WE als Handelnden dargestellt, im Deutschen wurde nur ein Beispiel dafür gefunden. Prozentual stehen dabei $6,32 \%$ aller englischen WE, die auf sechs Texte verteilt sind, dem einen deutschen WIR gegenüber, das $2,86 \%$ aller WIR ausmacht.

Dieses deutsche Beispiel referiert, genau wie die Hälfte der englischen Beispiele, exklusiv auf den Autor. Die restlichen englischen Fälle verteilen sich auf das TeamworkWIR (fünf Beispiele), das Gemeinschafts-WIR (zwei Beispiele) sowie jeweils einmal auf das pseudo-inklusive WIR und das Fachkreis-WIR. Die üblichste Umsetzung dieser Handlung ist die mit dem Verb find, daneben werden auch gelegentlich andere

\footnotetext{
${ }^{20}$ Vgl. dazu Ken Hylands Engagement-Strategie Reader Pronouns, Hyland (2005) und Kap. 3.2.2.
} 
Verben mit vergleichbarer Bedeutung verwendet wie get oder have sowie auch see oder conclude bzw. schließen auf.

(290) Yet by incorporating these ideas into the analysis of transnational standard setting in the areas of environment, labor, social justice, and human rights, we get an account that builds on but also pushes on some prominent sociological arguments about global governance. (Bartley 2007)

(291) What we see is that P-shift frequencies and likelihoods varied greatly in these groups, with some very common and others very uncommon. (Gibson 2005)

(292) Hence, when we direct our attention to the pure experimental comparison induced by MTO, we find that both groups not only ended up in very similar disadvantaged communities, they largely moved to the same exact communities. (Sampson 2008)

(293) Mit einer Irrtumswahrscheinlichkeit zwischen 5 und 10 Prozent können wir jedoch auf eine negative Beziehung zwischen ökonomischen Abstiegen des Partners und der Zweitgeborenenrate schließen: So weisen Frauen, deren Partner gegenüber dem Vorjahr einen Einkommensverlust von mindestens 5 Prozent hinnehmen mussten, eine um 29 Prozent geringere Übergangswahrscheinlichkeit zum zweiten Kind auf. (Brose 2008)

\section{Argumentieren}

Argumentationshandlungen sind nach der Begriffsbildung diejenigen, die am seltensten mit der ersten Person Plural kombiniert werden. Die fünf englischen Beispiele verteilen sich dabei auf vier Texte, die zwei deutschen werden auch in zwei verschiedenen Texten benutzt.

Prozentual machen diese Beispiele 1,75\% der englischen und 5,71 \% der deutschen Pluralpronomen aus. Drei der englischen Beispiele fallen in die exklusive Referenzkategorie nur Autor, während zwei zum Teamwork-WIR zählen. Die deutschen fallen ebenfalls in diese Kategorien bzw. das inklusive Beispiel ist nicht ganz eindeutig und fällt zwischen Teamwork und Fachkreis. Anders als bei der ersten Person Singular wird das Verb argue hier nicht benutzt, die Autoren greifen statt dessen auf Umschreibungen zurück, wie z. B. we assume for the sake of the current argument.

(294) On this basis we can judge whether the observed association is significantly smaller or larger than would have been expected given the null hypothesis of independence. (Gibson 2005)

(295) If we assume for the sake of the current argument that the selection model is reasonable and that the Robins inverse probability of treatment weighting (IPTW) method adjusts for baseline (wave 0) and time-varying confounding, the implications for MTO are significant. (Sampson 2008) 
(296) Dies gilt nicht nur für den Opernmarkt, sondern auch für den Bereich der Konzerte, wie wir mit einem anderen Datensatz nachweisen können; auch hier findet man eine hohe Standardisierung des Angebots. (Gerhards 2009)

\section{Begriffe bilden}

Aus der Kategorie Originator neuen Wissens wurde im Plural von drei amerikanischen Autoren sechsmal die Handlung der Begriffsbildung realisiert, was 2,11 \% der gesamten Pluralpronomen im englischen Korpus ausmacht. Im deutschen Korpus finden sich dafür keine Beispiele. Dabei fallen jeweils ein WE in die Referenzkategorien nur Autor, pseudo-inklusiv und Fachkreis, während zwei Beispiele nicht eindeutig zum Teamworkoder Fachkreis-WIR zugeordnet werden konnten.

Die Verben, die für die Beschreibung dieser Handlungen verwendet wurden, sind beispielsweise speak of, mean, term, understand. Das call, das häufig mit der ersten Person Singular kombiniert wurde, kam mit dem WIR nicht vor.

(297) The time therefore appears ripe for an interdisciplinary assessment of what we might term the "neighborhood question, experimental style." (Sampson 2008)

(298) Where citizens become convinced that such goals and principles do not (or no longer) provide a credible check on state action, we may speak of a system of "cynical domination." (Straughn 2005)

\section{Bezug auf Community-Wissen}

Mit diesen Handlungen stellt der Autor dar, was er für die geteilte Wissensbasis hält, von der er ausgeht bzw. was er als Community-eigenes Wissen annimmt, das den Ausgangspunkt definiert, von dem aus neues Wissen erarbeitet werden kann und muss. ${ }^{21}$ Diese Handlungen werden von 14 englischsprachigen und zwei deutschen Autoren mit dem WIR verwendet. Durch den generell inklusiven Bezug (alle 28 englischen Beispiele fallen unter das Fachkreis-WIR, die drei deutschen verteilen sich zwischen Teamwork- und Fachkreis-WIR) wird der Eindruck erweckt, dass diese Aussagen allgemeingültig seien.

Die verbale Umsetzung dieser Handlung erfolgt vorrangig über das Verb to know in unterschiedlichen Variationen, häufig auch mit dem Fokus darauf, was an Wissen noch nicht verfügbar ist, z. B. we do not know, we cannot know oder auch vergleichbar we cannot understand (Beispiel 300). Gelegentlich wird auch hervorgehoben, dass man etwas gelernt hat (Beispiel 301).

(299) To cite only a few well-known examples from a rapidly growing field of inquiry, we know that seemingly mundane state practices like taking censuses, making maps, and building museums (Anderson 1991) can become powerful

\footnotetext{
${ }^{21}$ Vgl. dazu Ken Hylands Engagement-Strategie Shared Knowledge, Hyland (2005) und Kap. 3.2.2
} 
instruments of state rule, as they help to constitute what they appear merely to represent. (Loveman 2005)

(300) The question holds obvious interest for those who study mechanisms of inequality in contemporary Russia because, as I show, we cannot understand stratification in Russia during the 1990s without taking into account inequality in getting paid. (Gerber 2006)

(301) These are all valid subjects of inquiry, and we have learned a great deal from their study, but in their haste to find either a substitute for the national bourgeoisie-namely, the state (Evans 1995) — or an explanation for its failure-namely, the worldsystem (Wallerstein 1991) and foreign investment (Bornschier, ChaseDunn, and Rubinson 1978)—sociologists all but entirely overlooked a simple point articulated by Jamaican economist Norman Girvan a quarter of a century ago: the mere fact that capitalists in developing countries tend to be "weak, dependent, and junior" does not mean that they are "objectively satisfied" with their situation (Girvan 1980, p. 451). (Schrank 2010)

(302) Martiniello (1997) observes that only when nonnationals enjoy full political rights in Europe will we be able to talk about a postnational citizenship. (Menjívar 2006)

(303) Diese zugegebenermaßen unvollständige Zusammenschau aktueller empirischer Befunde über die Erwerbserträge in Frauen-und Männerberufen belegt, dass wir, mit Ausnahme der Einkommensdimension, über wenig empirisch gesichertes Wissen verfügen. (Trappe 2006)

\section{Appell an Community}

Das Appellieren an die Community kommt, wie die Bezugnahme auf das CommunityWissen, wenig überraschend nur im Plural vor. Allerdings wird auch diese Handlung nur von den englischprachigen Autoren in ihren Texten vollführt. Die insgesamt 32 Beispiele, die sich auf zehn Texte verteilen, machen dabei 11,23\% aller hier relevanten englischen Pluralpronomen aus, wovon 23 auf den Fachkreis referieren, eines auf den Autor und den Leser (Teamwork-WIR) und neun nicht eindeutig in eine dieser beiden Kategorien eingeordnet werden können.

Der Appellcharakter an sich selbst und damit an die Community wird hier vorrangig durch die Modalverben must, shall sowie gelegentlich auch ought to bzw. need to ausgelöst, womit der Autor sich und der Gemeinschaft über das WIR eine Obligation zuspricht, bestimmte Dinge zu tun 22

(304) To see how colleges and universities facilitate or hinder the educational careers of potential matriculants, we must consider more systematically the organizational goals and values of postsecondary institutions. (Grodsky 2007)

\footnotetext{
${ }^{22}$ Vgl. dazu Ken Hylands Engagement-Strategie Directives, Hyland (2005) und Kap. 3.2.2.
} 
(305) Rather, consideration of this case serves a primarily heuristic purpose: it suggests why we ought to attend to the historical constitution of the state's symbolic power, illustrates how we can go about doing this, and indicates where the work of theory building could fruitfully begin. (Loveman 2005)

(306) If we are to understand the nature and effects of the new managerial regimes, we will have to explore the multiplicity of meanings they hold in the minds of the actors involved-not merely managers and executives, but also engineers, salaried employees generally, and hourly workers in various occupational positions-each of whom is sure to invoke collectively generated orientations and cultural practices that interact in mutually constitutive ways, mediating the effects of even the best-designed system of worker empowerment. (Vallas 2006)

(307) We need not doubt the sincerity of Wolf's commitment to reforming socialism to appreciate the brilliance of her performance. (Straughn 2005)

\section{Andere Forscherhandlungen}

Neben den oben beschriebenen Forscherhandlungen wurden in drei der englischen Texte noch 13 andere Handlungen mit dem WIR kombiniert, die zwar eindeutig zur Forscherrolle $\mathrm{zu}$ rechnen sind, aber nicht in eine der obigen Handlungskategorien fallen. Diese machen 4,56 \% aller WE-Vorkommen aus. 12 davon beziehen sich auf den Fachkreis und ein Beispiel fällt unter das Teamwork-WIR.

Wie beim ICH konnten hier auch keine typischen Verben herausgearbeitet werden, da die Handlungen hier sehr unterschiedlich ausfallen. Gemeinsam ist ihnen jedoch, dass sie sich auf Aufgaben und Tätigkeiten beziehen, die die Autoren im engeren oder weiteren wissenschaftlichen Kontext ausführen und/oder dass sie den Autor eindeutig als Wissenschaftler seines Fachbereichs charakterisieren. In Beispiel 308 muss das WE seine Soziologie, d.h. seine Forschung selbstverständlich publizieren, in Beispiel 309. nimmt der Autor darauf Bezug, dass das WE, wenn es ein bestimmtes Phänomen untersucht, dabei auch immer die eigene theoretische oder gar epistemologische Perspektive mitbringt (im weiteren Textverlauf wird dies am Beispiel des interaktionistischen gegenüber dem netzwerkanalytischen Standpunkt deutlich gemacht).

(308) Not all will wish to engage in what some may view as entrepreneurial activity, although, as Ericson (2005) astutely notes, we already publicize our sociology in the classroom, textbooks, research monographs, guest lectures, conferences, government reports, and Web sites. Nor will all wish to engage in the extra work required to disseminate research findings to other publics. (Vaughan 2006)

(309) Add to this fact scholarly specialization, and we have two very different ways of characterizing the world. (Gibson 2005) 


\subsection{Andere}

Neben den oben beschriebenen Verfasser- und Forscherhandlungen gibt es auch bei der ersten Person Plural eine weitere Rollenkategorie, in der alle anderen Rollen zusammengefasst werden. Diese enthält mit 36 englischen Beispielen 12,63 \% aller WEVorkommen, die sich auf sieben Texte verteilen, und daneben ein deutsches Beispiel $(2,86 \%)$. Dabei fällt die Hälfte der englischen Beispiele in die Kategorie des Gemeinschafts-WIRs, was der Rolle des Repräsentanten entspricht, in der der Autor sich und den Leser als Repräsentanten einer größeren Gruppe, ggf. sogar der ganzen Menschheit, darstellt. Hierunter ist auch das deutsche Beispiel zu rechnen (vgl. Beispiele 310 bis 312).

Daneben fallen in diese Rolle aber auch Berichte über bestimmte Forschungserlebnisse, in denen dann das WIR exklusiv zu lesen ist und den Autor und andere Personen umfasst, die in bestimmten Situationen am Forschungsprozess beteiligt waren (Beispiel 313). 14 der Fälle fallen daher in die Referenzkategorie Autor und andere. Die restlichen vier Vorkommnisse fallen unter das Fachkreis-WIR. Auch hier ist die Bandbreite der benutzten Verben sehr groß und es lassen sich keine typischen Verben ausmachen.

(310) We may want the dying to be unburdened by financial worries, but the ideal death today can be staggeringly expensive. (Quinn 2008)

(311) The rider, who represents our conscious processes, is the part of ourselves we know best—she can talk, reason, and explain things to our heart's content. (Vaisey 2009)

(312) Diese Trägerschichten und Gruppen genießen noch nicht jene Freiheiten, wie wir sie hier im Westen gewohnt sind. (Schwinn 2006)

(313) We talk a little about the work system, and both Gun and Kiner say they don't see any reason to rotate. (Vallas 2006)

\subsubsection{Zusammenfassung}

In diesem Kapitel wurde der Gebrauch der Pronomen insgesamt und in Hinblick auf die einzelnen Autorenrollen und die diesen Rollen zugeordneten Handlungskategorien verglichen. Grundsätzlich hat sich die Tendenz vorhergehender Studien, die sich mit dem Vergleich des Deutschen und des Englischen beschäftigt haben (Sanderson 2008a: Hutz|1997; Kresta|1995; Kussmaul|1978), dass die englischen Texte persönlicher geprägt sind als die deutschen, deutlich bestätigt.

Wie in Tabelle 5.1 auf S. 136 (hier wiedergegeben als Tab. 5.32) angegeben, enthält das englische Korpus, das dieser Untersuchung zugrundeliegt, deutlich mehr Vorkommen der ersten Person Singular und Plural als das deutsche, wohingegen das unpersönliche MAN in den deutschen Texten häufiger vorkommt. 


\begin{tabular}{|l|l|l|l|l|}
\hline Korpus & $\begin{array}{l}\text { Anzahl 1. Sg. } \\
\text { (auf 1000 Wör- } \\
\text { ter) }\end{array}$ & $\begin{array}{l}\text { Anzahl 1. Pl. } \\
\text { (auf 1000 Wör- } \\
\text { ter) }\end{array}$ & $\begin{array}{l}\text { Anzahl } \\
\text { MAN/ONE } \\
\text { (auf 1000 } \\
\text { Wörter) }\end{array}$ & $\begin{array}{l}\text { gesamt } \\
\text { (auf 1000 Wör- } \\
\text { ter) }\end{array}$ \\
\hline Englisch & $1311(3,47)$ & $285(0,75)$ & $119(0,32)$ & $1715(4,54)$ \\
\hline Deutsch & $105(0,56)$ & $35(0,19)$ & $300(1,59)$ & $440(2,34)$ \\
\hline
\end{tabular}

Tabelle 5.32: Übersicht alle Pronomen

Die quantitativen Ergebnisse für das deutsche Korpus sind vergleichbar mit Steinhoffs Expertentexten, in denen das ICH 0,43 Mal pro 1000 Wörter (Steinhoff 2007: 171), das WIR 0,57 Mal (Steinhoff|2007; 209) und das MAN 1,15 Mal (Steinhoff|2007; 210) benutzt wurde. Während also das ICH und das MAN in Steinhoffs Texten nicht ganz so häufig vorkamen, wurde das WIR in seinem Korpus etwas häufiger benutzt. Wenn man den Vergleich nur auf Steinhoffs linguistische Texte beschränkt, die in seinem Korpus mehr ICH-Vorkommen aufwiesen, als seine Texte aus der Literaturwissenschaft und der Geschichtswissenschaft, und die er wegen der deskriptiven und empirischen Herangehensweise der Linguistik als am ehesten vergleichbar mit den Gesellschaftswissenschaften ansieht (Steinhoff 2007 171), ergeben sich Werte von 0,74 (ICH), 1,03 (WIR) und 1,81 (MAN) (Steinhoff 2007 171, 209, 210). Damit benutzten die linguistischen Autoren alle Pronomen häufiger als die hier untersuchten Soziologen, wobei besonders ins Auge fällt, dass das WIR noch deutlich öfter vorkommt als das ICH. Und auch in der Literaturwissenschaft ist bei seinen Texten die Tendenz zu erkennen, dass das WIR $(0,59)$ und das MAN $(1,36)$ häufiger benutzt werden als das ICH $(0,35)$. Dies steht im Gegensatz zu den Ergebnissen dieser Untersuchung, in der das deutsche WIR noch deutlich seltener benutzt wird als das ICH.

Auch Ronald Krestas Ergebnisse zu den von ihm untersuchten linguistischen Aufsätzen zeigen einen deutlich höheren Gebrauch des WIR als die hier untersuchten, er kommt auf 1,33 deutsche und sogar 3,90 englische Pluralpronomen ${ }^{23}$ Diesen stehen 1,50 deutsche ICHs und 2,45 englische Is gegenüber. Das unpersönliche Pronomen konnte Kresta weder in seinen deutschen noch in seinen englischen Aufsätzen nachweisen (Kresta|1995: 187).

Tamsin Sanderson hat in ihrem Korpus aus mehreren geisteswissenschaftlichen Disziplinen (Sanderson 2008a: 65) insgesamt 454 deutsche und 1322 englische Singularpronomen der ersten Person sowie 779 deutsche und 1188 englische Pluralpronomen gefunden. Allerdings hat sie dabei alle Kasusformen zusammengefasst gezählt

\footnotetext{
${ }^{23}$ Kresta hat in seiner Arbeit keine normalisierten Werte für die von ihm untersuchten Items angegeben. Diese Zahlen beruhen auf Krestas Angaben zu seiner Korpusgröße, dessen jeweils 20 deutsche und englische Aufsätze im Schnitt 5605 bzw. 5065 Wörter umfassen (Kresta 1995 148). In diesen Aufsätzen hat er $275 \mathrm{Mal}$ das englische I und $152 \mathrm{Mal}$ das deutsche ICH (Kresta 1995 167) sowie 437 Mal WE und 135 Mal WIR (Kresta|1995 179) gefunden.
} 
(Sanderson 2008a: 110) und kam auf eine Verteilung von etwa 1,18 Mal ICH, 2,84 Mal I, 2,09 Mal WIR und 2,65 Mal WE auf 1000 Wörter 24 .

In Matthias Hutz' Korpus kamen insgesamt 82 Mal das WE und 79 Mal das WIR vor, das I dagegen nur 10 mal und das ICH gar nicht (Hutz 1997: 236). Da Hutz sein Korpus nicht anhand der Wortzahl beschreibt und auch keine normalisierten Werte angibt, sind diese Zahlen jedoch nicht mit den Ergebnissen dieser Untersuchung vergleichbar. Interessant ist aber trotzdem, dass das deutsche und das englische Pluralpronomen beinahe gleich häufig verwendet wurden. Zum MAN bzw. ONE macht Hutz nur Angaben bezüglich der Verteilung der Pronomen in Subjektposition im Verhältnis zu allen finiten Verbformen in seinem Korpus und kommentiert, dass

[d] as englische one im vorliegenden Korpus fast nicht vor[kommt] $(0,1 \%)$, wohingegen das entsprechende deutsche Pronomen immerhin bei 1,2 \% der finiten Verben die Subjektposition ausfüllt und damit häufiger vertreten ist als die Subjektpronomina 'ich/wir' zusammen. Zusammen mit dem geringeren Vorkommen bei der 1. Person und der hohen Frequenz von Passivkonstruktionen könnte dies ein Indiz dafür sein, daß die deutschsprachigen Texte insgesamt etwas unpersönlicher sind. (Hutz 1997; 238)

Darüber hinaus hat aber die spezifische Fragestellung dieser Arbeit auch weiterreichende Unterschiede in Hinblick darauf ergeben, wie die Autoren die Pronomen der ersten Person benutzen, um ihre Autorenidentität zu konstruieren. Die beiden relevanten Autorenrollen Verfasser und Forscher wurden zwar in beiden Korpora mit dem ICH und dem WIR dargestellt, aber in der Häufigkeit und auch den diese Rollen symbolisierenden Handlungen haben sich eindeutige Unterschiede ergeben.

Die Verfasserhandlungen werden in den englischen Texten von fast allen Autoren benutzt (24 von 25 ) und umfassen etwa ein Viertel (24,29 \%) der Handlungen in der englischen ersten Person Singular, während sie im Deutschen sogar fast die Hälfte $(44,76 \%)$ aller ICH-Handlungen stellen. Dagegen wurden 71,86 \% der englischen, aber nur 41,09 \% der deutschen Pronomen der ersten Person Singular mit Forscherhandlungen kombiniert. Bis auf eine Ausnahme haben alle englischen Autoren Forscherhandlungen benutzt, von den deutschen kamen diese Handlungen nur in 14 der 25 Texte vor. Und auch die Überprüfung mit dem Log-Likelihood-Test zeigte, dass die englischsprachigen Autoren sich in den meisten Fällen häufiger mit Handlungen in Verbindung bringen, die im engeren Sinne als „wissenschaftlich“ kategorisiert werden können. Dies gilt insbesondere für die Handlungen Datenaufbereitung/-analyse, Datenerhebung, Argumentation/Arguer und Ergebnisse, die alle mit p > 0,0001 deutlich häufiger im englischen Korpus vorkamen als im deutschen.

\footnotetext{
${ }^{24}$ Sanderson hat in ihrer Arbeit auf 10000 Wörter normalisiert, die von ihr angegebenen Zahlen sind ICH: 11,76; I: 28,41; WIR: 20,91; WE: 26,52 (Sanderson 2008a: 112).
} 
Im Plural werden im Deutschen die Verfasserhandlungen gegenüber den Forscherhandlungen bevorzugt. Während im englischen Korpus die Verfasserhandlungen nur 5,61 \% der Handlungen, die mit dem WE kombiniert werden, ausmachen, sind es im deutschen mit 34,29\% mit Abstand die häufigsten.

Die Ergebnisse für das Englische entsprechen den Beobachtungen von Tang und John (1999) und Hyland (2002a), dass diese Handlungen von englischsprachigen Wissenschaftlern gegenüber den Verfasserhandlungen als höherwertig eingestuft und werden (High stake). Verfasserhandlungen wie guide oder architect (Tang und John 1999) bzw. stating as purpose/goal (Hyland 2002a: 1101) werden eher von studentischen Autoren umgesetzt als die risikoreicheren Forscherhandlungen wie opinion-holder oder originator bzw. elaborating an argument und stating results/claims. Für einen Überblick über die Verteilung aller Autorenhandlungen in der ersten Person Singular und Plural s. Tabellen 5.2 .3 bis 5.2 .3 .

\begin{tabular}{|l|l|}
\hline Rolle & $\%$ \\
\hline Datenaufbereitung/ -analyse (F) & 25,76 \\
\hline verbale oder graphische Darstellung (V) & 12,74 \\
\hline Datenerhebung (F) & 14,30 \\
\hline Argumentation/ Arguer (F) & 11,27 \\
\hline Fokussierung der Forschungsfrage (F) & 4,86 \\
\hline Terminologieklärung (V) & 4,58 \\
\hline Danksagung (F) & 3,76 \\
\hline Hypothesen und Grundannahmen (F) & 3,48 \\
\hline Ergebnisse (F) & 3,30 \\
\hline Originator (F) & 3,30 \\
\hline Fokus des (Teil-)Textes (V) & 3,21 \\
\hline sonstige Metakommunikation (V) & 2,02 \\
\hline Andere & 1,92 \\
\hline andere Forscherhandlungen (F) & 1,83 \\
\hline Textstrukturierung (V) & 1,74 \\
\hline
\end{tabular}

Tabelle 5.33: prozentuale Verteilung auf die gesamte 1. Person Singular englisch 


\begin{tabular}{|l|l|}
\hline Rolle & $\%$ \\
\hline verbale oder graphische Darstellung (V) & 23,81 \\
\hline Danksagung (F) & 10,48 \\
\hline Andere & 9,71 \\
\hline Hypothesen und Grundannahmen (F) & 8,57 \\
\hline Datenaufbereitung/ -analyse (F) & 7,62 \\
\hline sonstige Metakommunikation (V) & 6,67 \\
\hline Fokus des (Teil-)Textes (V) & 5,71 \\
\hline Textstrukturierung (V) & 5,71 \\
\hline Argumentation/ Arguer (F) & 3,81 \\
\hline Originator (F) & 3,81 \\
\hline andere Forscherhandlungen (F) & 3,81 \\
\hline Terminologieklärung (V) & 2,86 \\
\hline Fokussierung der Forschungsfrage (F) & 0,95 \\
\hline Datenerhebung (F) & 0 \\
\hline Ergebnisse (F) & 0 \\
\hline
\end{tabular}

Tabelle 5.34: prozentuale Verteilung auf die gesamte 1. Person Singular deutsch

\begin{tabular}{|l|l|}
\hline Rolle & $\mathbf{\%}$ \\
\hline Methode/Datenerhebung/ -analyse & 26,67 \\
\hline Hypothesen und Grundannahmen & 15,90 \\
\hline Andere & 12,63 \\
\hline Appell an Community & 11,23 \\
\hline Bezug auf Community-Wissen & 9,82 \\
\hline Ergebnisse & 6,32 \\
\hline Verfasser gesamt & 5,61 \\
\hline unklar & 4,56 \\
\hline Fokussierung der Forschungsfrage & 4,21 \\
\hline Begriffsbildung & 2,11 \\
\hline Argumentation/ Arguer & 1,75 \\
\hline
\end{tabular}

Tabelle 5.35: prozentuale Verteilung auf die gesamte 1. Person Plural englisch 


\begin{tabular}{|l|l|}
\hline Rolle & \% \\
\hline Verfasser gesamt & 34,29 \\
\hline Methode/Datenerhebung/ -analyse & 25,21 \\
\hline Hypothesen und Grundannahmen & 20,00 \\
\hline Bezug auf Community-Wissen & 8,57 \\
\hline Argumentation/Arguer & 5,71 \\
\hline Ergebnisse & 2,86 \\
\hline Andere & 2,86 \\
\hline Appell an Community & 0 \\
\hline Fokussierung der Forschungsfrage & 0 \\
\hline Begriffsbildung & 0 \\
\hline unklar & 0 \\
\hline
\end{tabular}

Tabelle 5.36: prozentuale Verteilung auf die gesamte 1. Person Plural deutsch

Die erste Person wird in beiden Sprachen nur an ausgewählten Stellen eingesetzt und sollte offenbar nicht zu häufig benutzt werden, also müssen die Autoren sorgfältig auswählen, wann und wo sie sich in ihren Text so deutlich einbringen. Die unterschiedliche Gewichtung der einzelnen Handlungen zeigt, wie unterschiedlich das symbolische Kapital des „Eigenanteils“ bzw. der eigenen Arbeit des Wissenschaftlers in den beiden untersuchten Wissenschaftskulturen ist. Während die eigene Forschungsleistung von den US-amerikanischen Autoren durch den Gebrauch der ersten Person deutlich betont wird, wird sie von den deutschen Autoren nicht in den Mittelpunkt gerückt, sondern statt dessen über unpersönliche oder sogar passive Wendungen ausgedrückt. Dies fällt im direkten Vergleich besonders im Fall der Handlungen der Datenerhebung auf.

Darüber hinaus scheint aus anglo-amerikanischer Perspektive die direkte Interaktion des Autors mit seinen Lesern offenbar eine größere Rolle als im deutschen zu spielen, was sich auch daran zeigt, dass dieses Thema in der Forschung intensiv bearbeitet wird, wie die genannten Arbeiten zu Voice und Stance and Engagement im wissenschaftlichen Schreiben zeigen (s. Kap. 3.2). Diese Beobachtung ist auch konsistent mit früheren Beobachtungen zu einer ausgeprägten reader-orientation von englischsprachigen wissenschaftlichen Autoren gegenüber einer stärkeren writer-orientation in den deutschsprachigen Wissenschaften (vgl. z. B. Clyne|1991. 382-383).

Beispielsweise scheinen die amerikanischen Autoren, zumindest auf den ersten Blick, ihre Leser stärker und v. a. direkter in ihre Argumentation einzubeziehen, was sich z. B. an einigen Handlungen der verbalen und graphischen Darstellung zeigen lässt. Der Gebrauch der Verben offer und propose bindet den Leser deutlich stärker in die Argumentation mit ein als andere Verben describe, illustrieren, was als deutliche Engagement-Strategie gewertet werden kann. Die deutschen Autoren neigen hier eher zu 
Abschwächungen anhand von Modalverben (Hedges), was in Hylands Kategorisierung am ehesten als Ausprägung von Stance gewertet würde (Hyland 2008: 10). Im konkreten Fall der deutschen Beispiele verbaler und graphischer Darstellung (Beispiele 163 und 164 von S. 153 , hier wiedergegeben als Beispiele 314 und 315) ist diese Abschwächung aber vermutlich ebenfalls eher als Engagement-Strategie zu werten, da die Formulierungen möchte ich [...] skizzieren und möchte ich erläutern im Deutschen ähnlich wie das Englische offer oder propose die Möglichkeit suggerieren, dass der Leser dem Autor diese Bitte auch abschlagen und damit Einfluss auf die Argumentation nehmen könnte.

(314) Daher möchte ich abschließend skizzieren, wie eine Neuorientierung der typologischen Lebensstilforschung aussehen könnte, die die Voraussetzungen für eine systematischere empirische Untersuchung ihrer zentralen Behauptungen schafft. (Otte 2005)

(315) Diese Zusammenhänge möchte ich etwas genauer am Beispiel der Ausbreitung von McDonalds in Ostasien erläutern. (Schwinn 2006)

Dies zeigt, dass der rein linguistische Vergleich sprachlicher Phänomene, auch wenn er auf der Ebene der linguistischen Funktionen stattfindet, nicht immer ausreicht, um den (wissenschaftlichen) Sprachgebrauch zu erklären. Es müssen darüber hinaus auch die soziale bzw. soziokulturelle und hier insbesondere die interaktive funktionale Ebene berücksichtigt werden. Beispielsweise wäre eine Einteilung der Autorenhandlungen anhand von Interaktions- und Höflichkeitsstrategien, ähnlich wie Hylands Kategorien von Stance and Engagement oder Tang und Johns Risikohierarchie, sicherlich (nicht nur) für die Schreibdidaktik von Nutzen. Allerdings bedarf es hierfür noch weiterer zusätzlicher Untersuchungen. Insbesondere müssten dann auch umfangreiche Daten wie Interviews und Expertenbefragungen mit Wissenschaftlern einerseits zu deren eigenem Schreibverhalten und andererseits zu ihren Einschätzungen von Texten anderer Autoren erhoben werden.

\subsection{Fallstudie Vaughan}

Der Text von Diane Vaughan (Vaughan 2006) ist ein Sonderbeispiel, das im Folgenden eingehender untersucht werden soll. Formal entspricht er voll und ganz einem typischen wissenschaftlichen Artikel, er enthält beispielsweise ein Abstract, Zwischenüberschriften sowie Zitate und Literaturliste. Auch inhaltlich sind die regulären $\mathrm{Ab}$ schnitte eines wissenschaftlichen Fachartikels erkennbar.

Allerdings ist dieser Artikel für diese Arbeit besonders interessant, da hier die eigene wissenschaftliche Identität der Autorin das Thema bildet. Die Autorin reflektiert ihre eigenen Ansichten und ihr Handeln als Soziologin in einem spezifischen For- 
schungszusammenhang. Dabei verwendet sie eine sehr untypische Darstellungsweise, ihr Artikel gleicht einem Erlebnisbericht oder einer Reportage und ist, dem Thema entsprechend, aus einer sehr persönlichen Perspektive geschrieben. Diese narrative Darstellungshaltung wurde in verschiedenen Untersuchungen als eindeutig unwissenschaftlich identifiziert (z. B. Tang und John|1999: Hyland|2002a; Steinhoff|2007). Die Frage, die sich hier stellt, ist, wie die Autorin in ihrem Artikel die erste Person Singular benutzt. Eine weitere Frage, die in diesem Rahmen jedoch nicht vollständig beantwortet werden kann, ist darüber hinaus auch, ob es die Autorin trotz ihres untypischen ICH-Gebrauchs schafft, ihre Glaubwürdigkeit als wissenschaftliche Autorin aufrechtzuerhalten und ggf. sogar noch zu stärken.

Diane Vaughan ist ein mehrfach mit Preisen ausgezeichnetes Mitglied der US-amerikanischen und weltweiten soziologischen Community und eine erfahrene wissenschaftliche Autorin, deren Werke auch über die wissenschaftliche Gemeinschaft hinaus eine Leserschaft gefunden haben. Für ihre Analyse der Challenger-Katastrophe (Vaughan 1996), die auch die theoretische Basis für die im hier untersuchten Artikel vorgestellte Analyse bildet, ist sie u. a. mit dem Rachel Carson Prize der Society for Social Studies of Science (1998) und dem Robert K. Merton Book Award (Science, Knowledge, and Technology Section) der American Sociological Association (1996) ausgezeichnet worden. Weiterhin war sie für diese Arbeit im Jahr 1996 für den National Book Award sowie den Pulitzer-Preis in der Kategorie Non-Fiction nominiert25. Darüber hinaus wurde ihr im Jahre 2006 von der American Sociological Association der Public Understanding of Sociology Award zugesprochen. Sie hat ihre gesamte akademische Karriere im Fach Soziologie vollzogen und ist seit 1996 Professorin für Soziologie, zunächst am Boston College und aktuell an der Columbia University ${ }^{26}$

Man kann also mit großer Sicherheit davon ausgehen, dass sie die Schreibkonventionen ihres Faches sehr gut kennt und die hier beschriebenen Abweichungen bewusst und gewollt vollzogen hat. Insofern ist der hier untersuchte Text ein gutes Beispiel dafür, dass wissenschaftliche Schreibkonventionen, wie alle soziokulturellen Konventionen, nicht als deterministisch aufgefasst werden dürfen, sondern nur ein grobes Gerüst bilden, innerhalb dessen die individuellen Autoren abhängig von ihren eigenen Präferenzen und auch den jeweiligen Themen, die sie in ihren Texten bearbeiten, ihren eigenen Stil ausbilden können. Individuelle Entscheidungsmöglichkeiten innerhalb der wissenschaftskulturellen Konventionen sind also immer vorhanden und können von entsprechend erfahrenen Autoren auch in vollem Ausmaß eingesetzt werden.

Bei der folgenden Auswertung des ICH-Gebrauchs in Diane Vaughans Text werden vorrangig diejenigen Vorkommnisse betrachtet, mit denen sie soziale Rollen umsetzt, die weniger typisch für wissenschaftliche Texte sind und die in den anderen Korpustexten nicht oder nur in Einzelfällen benutzt wurden. Doch natürlich hat die Autorin

\footnotetext{
${ }^{25}$ http://sociology.columbia.edu/files/sociology/vaughan_cv.pdf. Zugriff 03.10.2013

${ }^{26}$ http://sociology.columbia.edu/files/sociology/vaughan_cv.pdf. Zugriff 03.10.2013
} 
auch typische Handlungen umgesetzt, für die hier zunächst einige Beispiele aufgeführt werden.

Wie in anderen Texten auch tritt Diane Vaughan in ihrem Text ebenfalls als die Verfasserin in Erscheinung, allerdings übernimmt sie diese Rolle nur selten. In Beispiel 316 schreibt sie sich ein Verb der verbalen Darstellung zu (discuss) und verweist damit vor auf ein späteres Kapitel (later).

(316) The board's composition (ultimately significant in the framing discourse of the board's report, as I will discuss later) was a product of history and politics. (Absatz 17)

Handlungen, mit denen sie sich als Forscherin charakterisiert, benutzt Vaughan dagegen deutlich häufiger. Während sie in Beispiel 317 ihren (ursprünglichen) Forschungsfokus beschreibt, stellt sie sich in den folgenden Beispielen als die Person dar, die entscheidet, welche Daten sie für ihre Forschung verwendet, und die diese Daten dann erhebt (Beispiele 318 bis 322). Die Beispiele 323 bis 325 zeigen sie dann als diejenige Person, die ihre Daten analysiert.

(317) Never had I intended such an extended diversion from my ongoing research, but now hooked by their puzzle and process, I agreed. (Absatz 31)

(318) My data are constituted in my participation at the CAIB and NASA and a chronology and content analysis of over 1,200 e-mails I received related to the Columbia accident. (Absatz 9)

(319) Also useful were $4 \times 6$ cards on which I recorded each telephone contact by the media and others by date and phone numbers, taking notes on questions asked and new information I received. (Absatz 9)

(320) Using the Simmel-based method of analogical theorizing that guided my Challenger analysis (Vaughan 1992), I converted my accumulating data on the accident into a more systematic comparison, beginning a focused revisit to the site of my previous study. (Absatz 23)

(321) To identify both similarities and differences, I began systematically sorting my data into institutional, organizational, and decision-making levels of analysis. (Absatz 24)

(322) Because I was working with data from some interviews given under conditions of anonymity and considered privileged communication by the board, I was asked to sign a nondisclosure agreement. (Absatz 50)

(323) I analyzed all newspaper and wire accounts relevant to the accident and to the CAIB and NASA activities available via the CAIB clipping service, and I examined Internet documents. (Absatz 9) 
(324) Finally, I compared succeeding versions of the CAIB report chapters addressing the social causes of the accident. (Absatz 9)

(325) Working inductively from this ethnographic account, I identify the mechanisms that enabled sociological concepts and theory to travel across the discipline's boundary to become meaningful in the public and policy realms. (Absatz 9)

Noch deutlich häufiger als in den anderen Texten schreibt sich die Autorin in diesem Text auch andere Rollen zu, die nicht direkt mit dem Verfassen des Textes oder dem Forschungsprozess zusammenhängen. Beispielsweise verweist sie immer wieder auf ihre Beteiligung an anderen Forschungsprojekten, insbesondere natürlich die Forschungsarbeit, aufgrund derer sie als Expertin für die Untersuchung des in diesem Text behandelten Ereignisses beauftragt wurde (Beispiel 326). In Beispiel 327 dagegen beschreibt sie sich im Kontext eines anderen, neuen Projekts, womit sie ebenfalls ihre Rolle als (vielbeschäftigte) Wissenschaftlerin betont, die regelmäßig neue Forschungsprojekte durchführt.

(326) I draw upon my experiences from February 2003 through February 2004, reconstructing them chronologically in an ethnographic account of the four stages of post-Columbia rituals of risk and error: [...] (Absatz 9)

(327) I was deep into a new project, so was caught off guard by the sudden NASArelated intrusion of media and interested others. (Absatz 15)

Darüber hinaus setzt sie sich in Beziehung zu anderen von ihr geschriebenen Texten und stellt sich so aus einer weiteren Perspektive als wissenschaftliche Autorin dar. Hier betont sie nicht, wie in der Rolle als Verfasserin, ihre Funktion als die kreative und organisierende Instanz hinter ihren Texten, sondern sie schreibt sich die (wissenschaftliche) Tätigkeit des Schreibens und Veröffentlichens zu. Im Kontext einer auf dem Konzept des publish-or-perish beruhenden Wissenschaftskultur stellt dies sicher eine zwar recht subtile, aber dennoch massive Eigenwerbung dar (s. auch Kap. 5.4).

(328) Because I had written a book on the Challenger accident (Vaughan 1996), I was viewed as an expert on NASA and shuttle accidents. (Absatz 3)

(329) Soon, copies of it and a theoretically true but de-jargonized management journal condensation that I wrote (Vaughan 1997) were circulating in the offices of the CAIB and its staff. (Absatz 28)

Eine weitere wissenschaftliche Tätigkeit, in dem Sinne, dass sie zum Arbeitsalltag der meisten Wissenschaftler gehört, ist die Lehre. Zwar wird die eigene Rolle als Lehrperson in wissenschaftlichen Forschungsartikeln von den Autoren üblicherweise nicht thematisiert ${ }^{27}$, Vaughan aber stellt sich in ihrem Artikel dennoch auch als Lehrperson

\footnotetext{
${ }^{27}$ In anderen, eher didaktischen Textsorten, wie einem Einführungsartikel oder einem Lehrbuch steht diese Rolle vermutlich mehr im Zentrum.
} 
dar und baut diese Facette ihrer wissenschaftlichen Persona aus. Dadurch gewinnt sie zusätzlich an Glaubwürdigkeit und Autorität, da sie sich so nochmals deutlich im professionellen soziologischen Umfeld verortet und dabei auch ihre produzierende und formende Funktion innerhalb dieses Kontextes betont.

(330) What I was teaching was the sociological perspective, using the theory and concepts that explained Challenger. (Absatz 20)

Diese Funktion steht auch in den folgenden Beispielen im Vordergrund. Hier stellt sie sich als Wissenschaftlerin bzw. konkret als Soziologin dar, indem sie Bezug zu soziologischen Themen und Theorien herstellt (Beispiel 331), ihren eigenen Werdegang anhand typischer Stationen (Graduate Student) nachzeichnet (Beispiel 332) oder sich, wie auch in Beispiel 330, typische professionelle Tätigkeiten zuschreibt (Beispiele 333 bis 335.

(331) With cold hands and keen awareness of this as the most literal deployment of Goffman's (1959) frontstage and backstage distinction that I had ever experienced, we passed through the parted curtain into the bright lights and, seated at tables in symbolic adversarial opposition, snapped into appropriate roles. (Absatz 32)

(332) I did participate in interviews and other media activities because while teaching as a graduate student I saw how sociology could challenge or even alter people's understanding of their own and others' lives. (Absatz 19)

(333) I self-defined as doing professional sociology in another setting: research, data analysis, and teaching; public sociology relevant to policy; not political activism working for institutional and organizational change. (Absatz 93)

(334) I was not trained to go beyond sociological principles and examples to implementing change. (Absatz 94)

(335) Not only do the four sociologies merge into blurred genres in my experience, as I was often doing all or several at once, but so do the two types of public sociology. (Absatz 97)

Neben diesen direkten Selbstzuschreibungen stellt sich Vaughan aber auch in der AuBenperspektive dar und beschreibt, wie sie als Expertin, Beraterin oder Gutachterin von verschiedenen Seiten wahrgenommen und konsultiert wurde bzw. wird.

(336) Because I had written a book on the Challenger accident (Vaughan 1996), I was viewed as an expert on NASA and shuttle accidents. (Absatz 3)

(337) I was consulted by the press, called to testify before the CAIB, invited to join the board as a consultant and staff researcher, and worked on the official report, authoring a chapter. (Absatz 3) 
(338) For Space Center City and East Coast journalists and National Public Radio, I became a regular source. (Absatz 22)

(339) In late March, I was invited to testify in the CAIB public hearings in Houston. (Absatz 23)

Ein wesentlicher Unterschied $\mathrm{zu}$ den anderen hier untersuchten wissenschaftlichen Texten, und auch zu der generellen Auffassung von „Wissenschaftlichkeit“ in der Forschung zu wissenschaftlichem Schreiben (z. B. Steinhoff 2007; Hyland 2001b) sind die umfassenden narrativen Passagen, die dieser Text enthält. Diese sind in erster Linie sicherlich in dem methodischen Ansatz, den die Autorin in ihrer Untersuchung verfolgt hat, begründet. Ethnographische Studien dieser Art erfordern von denjenigen, die sie durchführen, in der Regel ein deutlich stärkeres persönliches Einbringen in den Forschungskontext als beispielsweise quantitative Erhebungsverfahren ${ }^{28}$ Dadurch erhalten natürlich nicht nur die Auswertungen und Interpretationen, sondern auch der ganze Prozess der Datenerhebung eine deutlich persönlichere Färbung (vgl. auch den Korpustext Viterna 2006).

Dennoch geht Diane Vaughan hier eindeutig über die für die Darstellung ethnographischer Studien übliche persönlichere Perspektive hinaus und thematisiert ihre eigenen Erlebnisse nicht nur nicht neutral, sondern bringt immer wieder bewusst ihre eigenen Emotionen, Erwartungen und Reaktionen mit ein. Diese Darstellungsweise wurde von Steinhoff (2007) und Hyland (2001b) als eindeutig nicht wissenschaftlich und als Hinweis auf einen ungeübten studentischen Autor beschrieben. Hier zeigt sich, dass diese Charakterisierung zu kurz gefasst ist, da, in den richtigen Händen, auch dieser Stil der Wissenschaftlichkeit eines Textes nicht notwendigerweise schaden muss. In den folgenden Beispielen 340 bis 344 beschreibt die Autorin sich und ihre eigene Position aus ihrer Perspektive und thematisiert dabei Umstände auf eine sehr persönliche und berichtende Art, wobei deren direkter Informationsgehalt für die Forschung und die Ergebnisse sich nicht immer auch direkt erschließt. Weiterhin thematisiert sie neben den tatsächlichen Vorkommnissen und ihren Aktivitäten auch immer wieder emotionale Aspekte (Beipiele 345 bis 349).

(340) About 10:30 a.m. on Saturday, February 1, as I watched television replays of Columbia's tragic disintegration unfold, I began receiving phone calls and emails about the accident. (Absatz 15)

(341) I had no experience as an expert witness, let alone as a participant in an official government investigation of this scale. (Absatz 25)

\footnotetext{
${ }^{28}$ In Disziplinen, in denen diese Methoden häufig verwendet werden, gehören Abschnitte und ganze Teiltexte in erzählendem Stil inzwischen zu den allgemein anerkannten Darstellungstechniken (Auer und Baßler 2007a 21).
} 
(342) Unable to proceed unescorted, I was assigned an aide, a recent political science Ph.D. who became my informant, tutoring me about CAIB members, the investigation, and the hearings. (Absatz 27)

(343) At 8:00 a.m., I attended the "stand-up" briefing, a military tradition. (Absatz 28)

(344) I had been responding to the news; now I became the news. (Absatz 25)

(345) Unaware of the extent of the book's influence on the CAIB's thinking, I arrived in Houston anxious about my interrogation. (Absatz 25)

(346) I was astonished. (Absatz 16)

(347) Nearing the third hour, I felt it would never be over. (Absatz 41)

(348) Working through the data with them, I suddenly felt at home. (Absatz 29)

(349) The insights I gained at Wye about NASA headquarter's position on change made me fear I would be used for symbolic purposes only and that my ideas would be abused or not used at all. (Absatz 94)

In den folgenden Beispielen werden narrative Passagen mit der Selbstbeschreibung als Soziologin kombiniert.

(350) With cold hands and keen awareness of this as the most literal deployment of Goffman's (1959) frontstage and backstage distinction that I had ever experienced, we passed through the parted curtain into the bright lights and, seated at tables in symbolic adversarial opposition, snapped into appropriate roles. (Absatz 32)

(351) "How do you know you can do that?" he asked. "I'm trained to do that," I said. (Absatz 49)

(352) Not only was my research integral to their work, but I also was an embedded sociologist, seduced by doing professional sociology as a full participant in a like-minded group. (Absatz 91)

(353) I did not see how the transition to full CAIB member gradually changed my public sociology. (Absatz 92)

(354) Consider also that a summer of 12-hour days left no time for writing the field notes that build in daily reflexivity, I was isolated from colleagues who might provide criticism, and being useful is seductive in its own right. (Absatz 93)

(355) The tensions between professional sociology and public sociology began immediately, as I set aside my ongoing field research, juggled teaching and departmental responsibilities, and deferred professional writing and reviewing commitments. (Absatz 89) 
Die vorangehenden Kapitel haben deutlich gemacht, dass es für die Möglichkeiten, über die erste Person Singular die eigene Identität als wissenschaftlicher Autor zu konstruieren, offensichtlich wissenschaftskulturell bedingte Konventionen gibt. Der hier beschriebene Text zeigt aber, dass diese Konventionen bei Weitem nicht so fest und eindeutig sind, wie es den Anschein hat. Insbesondere erfahrene Autoren, die sicherlich aufgrund ihrer eigenen Position innerhalb der wissenschaftlichen Community auch über ein gewisses Sebstbewusstsein verfügen, sind gut in der Lage, ihre eigene wissenschaftliche Persona auf eine sehr kraftvolle und autoritative Weise in ihren Text einzubringen, obwohl oder vermutlich sogar gerade weil sie bewusst gegen allgemeine Tendenzen verstoßen. Diane Vaughan liegt mit über 200 Vorkommnissen der ersten Person Singular weit über dem Durchschnitt auch der tendenziell persönlicheren englischen Texte dieses Korpus und verstößt damit deutlich gegen das auch im englischsprachigen Raum verbreitete Objektivitätsgebot wissenschaftlicher Texte (s. z. B. Hinkel 1999: Hyland 2001b:90). Darüber hinaus berichtet sie anhand weiter narrativer Passagen über ihre Forschung und benutzt deutlich emotional und affektiv geprägte Sprache sowie wörtliche Rede in ihrem Text. Doch trotz alledem steht bei der Lektüre ihres Texte nie in Frage, ob ihr Text tatsächlich ein wissenschaftlicher Text ist und ob sie sich entsprechend glaubwürdig als wissenschaftliche Autorin darstellt. Es wäre sicherlich lohnenswert, diesen und auch andere Texte, die so deutlich gegen die sowohl wissenschaftslinguistisch beschriebenen als auch intuitiv von Vertretern der Disziplinen anerkannten Textkonventionen verstoßen, tiefer gehend zu analysieren und die weiteren Faktoren zu untersuchen, die die wissenschaftliche Voice der Autoren ausmachen, was aber im Rahmen dieser Arbeit nicht erfolgen kann.

\subsection{Impression Management: ein sozialpsychologischer Ex- kurs zur Selbstdarstellung}

Der grundlegende theoretische Rahmen dieser Arbeit ist linguistisch und soziologisch geprägt, insbesondere die Diskussion um Identität und Selbstdarstellung anhand der Übernahme und Selbstzuschreibung sozialer Rollen über Handlungen spiegelt eine deutlich soziologische Perspektive wider. Nun kann man jedoch argumentieren, dass die Frage "Wer bin ich?" bzw. „Wer will/kann/darf ich in dieser Situation sein?" auch eine nicht unwesentliche (sozial)psychologische Komponente enthält. Aus diesem Grund soll hier ein kurzer Abriss über den sozialpsychologischen Ansatz des Impression Management gegeben werden, um zu zeigen, dass sich die oben diskutierten Beispiele und das verwendete Kategoriensystem auch aus dieser Perspektive stützen lassen. 
Die Impression-Management-Theorie beschäftigt sich mit der Frage, wie sich Menschen in sozialen Interaktionen selber darstellen und welche Techniken dabei verwendet werden.

Individuen kontrollieren (beeinflussen, steuern, manipulieren etc.) in sozialen Interaktionen den Eindruck, den sie auf andere Personen machen. (Mummendey u.a. 1995, 111)

Als wichtige Vorläufer bzw. philosophische und soziologische Grundlagen für diesen Ansatz nennt Mummendey (Mummendey 2002; Mummendey u. a. 1995: Kap. 6) den Symbolischen Interaktionismus nach George Herbert Mead und die Arbeiten von Erving Goffman (z. B. Goffman 1959, 1971), wobei er hier insbesondere die Theatermetapher hervorhebt (Mummendey u.a. 1995. 118-119). Daneben nennt er von pychologischer Seite die Arbeitsfelder der Selbstkonzeptforschung und der Sozialen Erwïnschtheit (social desirability) als weitere Grundlagen (Mummendey 2002: 215-216). Die empirische Überprüfung anhand von experimenteller Erforschung der Impression Management-Strategien und -Techniken begann Mummendey zufolge Anfang der 1970er Jahre (Mummendey u.a. 1995, 122) und in der aktuellen Forschung stehen häufig insbesondere wirtschaftspsychologische Fragestellungen sowie die Erforschung von Selbstpräsentationen im Internet im Vordergrund (z. B. Graffin u. a. 2011; Jackson und Lilleker 2011; Merkl-Davies u. a. 2011; Merkl-Davies und Brennan 2011; Rosenberg und Egbert 2011).

Basierend auf den Ergebnissen der sozialpsychologischen Forschung spricht Mummendey (Mummendey u.a. 1995) von "Selbstdarstellungstechniken" und unterscheidet in seinem Forschungsüberblick zwischen positiven und negativen Techniken der Selbstdarstellung (s. auch Fußnote 33 auf S. 207). "Positiv“ bedeutet in diesem Zusammenhang, dass durch diese Techniken der eigenen Person eher positive Merkmale zugeschrieben werden, mit denen sich jemand in "günstiger, sich selbst erhöhender und sozial gebilligter Weise“ darstellt. „Negativ“ meint dagegen, dass man sich „in eher ungünstiger, sich selbst herabsetzender und sozial weniger gebilligter Weise präsentiert" (Mummendey u. a. 1995, 140). Als eher positive Techniken stellt er folgende dar (Mummendey u. a. 1995: 140-141):

- Eigenwerbung betreiben (self-promotion)

- Hohe Ansprüche signalisieren (entitlements)

- Hohes Selbstwertgefühl herausstellen (self-enhancement) und übertreiben (overstatement)

- Sich über Kontakte aufwerten (BIRGing = basking in reflected glory) und sich über Kontakte positiv abheben (boosting)

- Kompetenz und Expertentum signalisieren (competence, expertise)

- Beispielhaft erscheinen (exemplification)

- Attraktivität herausstellen (personal attraction) 
- Hohen Status und Prestige herauskehren (status, prestige)

- Glaubwürdigkeit und Vertrauenswürdigkeit herausstellen (credibility, trustworthiness)

- Offenheit hervorkehren (self-disclosure)

- Sich beliebt machen, sich einschmeicheln (ingratiation, other-enhancement) ${ }^{29}$ $\mathrm{Zu}$ den in der Forschungsliteratur beschriebenen eher negativen Techniken zählt er folgende (Mummendey u. a. 1995: 141):

- Entschuldigen, Abstreiten von Verantwortlichkeit (apologies, excuses), Rechtfertigen (justification, accounts) in mißlichen Lagen (predicaments)

- Widerrufen, ableugnen, dementieren, vorsorglich abschwächen (disclaimers)

- Sich als unvollkommen darstellen (self-handicapping)

- Understatement

- Hilfsbedürftig erscheinen (supplication)

- Symptome geistiger Erkrankung zeigen

- Bedrohen, einschüchtern (intimidation)

- Abwerten anderer (blasting) 30

Zwar basieren diese Kategorien überwiegend auf Untersuchungen zu Face-to-faceInteraktionen und sind $\mathrm{m}$.W. noch nicht im Rahmen von wissenschaftlichem Schreiben betrachtet worden, aber da, wie in Kap. 2.1 ausgeführt, auch das wissenschaftliche Schreiben als soziale Interaktion verstanden werden muss und diese Techniken der Selbstdarstellung in allen sozialen Interaktionen angewandt werden können, ist anzunehmen, dass zumindest einige dieser Techniken in den hier untersuchten wissenschaftlichen Artikeln identifiziert werden können. Beispielsweise kann man sicherlich erwarten, Signale von Kompetenz und Expertentum des Autors in einem wissenschaftlichen Artikel zu finden, und auch das Herausstellen von Glaubwürdigkeit ist ein wesentlicher Bestandteil guten wissenschaftlichen Schreibens. Daneben ist es eine häufige Technik, die eigene Unvollkommenheit (bzw. zumindest die der eigenen Forschung) zu betonen und auch vorsorgliches Abschwächen (hedging) im wissenschaftlichen Schreiben ist schon mehrfach beschrieben und untersucht worden (s. z. B. Graefen 2000: Hyland 1998). Andere Techniken, wie z. B. die eigene „Attraktivität herausstellen“ oder „Symptome geistiger Erkrankung zeigen“ sind dagegen weniger wahrscheinlich.

Die Frage, die sich im Folgenden stellt, ist, inwiefern diese Techniken in den untersuchten Texten mit der ersten Person Singular realisiert werden ${ }^{31}$ Eine eindeutige $\mathrm{Zu}$ ordnung dieser Selbstdarstellungstechniken ist dabei jedoch sicherlich nicht zu treffen,

\footnotetext{
${ }^{29}$ Diese Technik wird später in seinem Text als nicht eindeutig zuordenbar vorgestellt (vgl. Mummendey u.a. 1995 152).

${ }^{30}$ In der folgenden exemplarischen Auswertung werden nur ausgewählte Techniken berücksichtigt.

${ }^{31}$ Sicherlich wird es auch hinlänglich Beispiele geben, die nicht über die erste Person realisiert werden, die aber trotzdem eindeutig als Selbstdarstellung des Autors gewertet werden können. Diese sind jedoch nicht Teil der hier beschriebenen Untersuchung.
} 
vielmehr ist davon auszugehen, dass einige Techniken sich in verschiedenen sprachlichen Mitteln bzw. Handlungen widerspiegeln oder aber, dass eine Aussage mehreren (ähnlichen) Strategien zugeordnet werden kann. Außerdem repräsentiert nicht jede der hier untersuchten Vorkommen der ersten Person Singular auch eine dieser expliziten Techniken der Selbstdarstellung. Das Ziel soll hier also nur sein zu zeigen, dass diese Techniken im wissenschaftlichen Schreiben auch eine Rolle spielen können und dass es in den untersuchten Texten Passagen gibt, die im Sinne des Impression Management gedeutet werden können. Damit soll die Annahme, dass auch in wissenschaftlichen Artikeln als einer Form der sozialen Interaktion die Selbstdarstellung des Autors und die Konstruktion einer angemessenen wissenschaftlichen Identität eine wesentliche Rolle spielen, auf eine breitere und sozialpsychologisch begründete Basis gestellt werden.

Parallel zu den oben beschriebenen Rollen, die ein wissenschaftlicher Autor in seinem Text einnehmen kann, möchte ich im Folgenden exemplarisch einige Techniken des Impression Management näher untersuchen und herausarbeiten, ob und wie diese Techniken auch in wissenschaftlichen Texten eingesetzt werden. Dabei werde ich mich auf Beispiele beschränken, in denen der jeweilige Autor durch den Gebrauch der ersten Person eindeutig zu identifizieren ist. Das heißt natürlich nicht, dass diese Techniken nicht auch durch andere sprachliche Mittel umgesetzt werden könnten.

Es ist hier nicht Ziel, diese Techniken der Selbstdarstellung in den untersuchten Texten eins zu eins wiederzufinden. Dies ist aller Wahrscheinlichkeit nach auch nicht möglich. Vielmehr soll gezeigt werden, dass einige dieser Techniken sich in den Texten wiederfinden lassen bzw. einige der untersuchten Passagen eine Interpretation im Rahmen der Impression Management-Theorie zulassen.

Im Folgenden gebe ich einen kurzen Überblick über die hier relevanten Techniken der Selbstdarstellung (vgl. die Übersicht auf S. 202). Dieser Überblick basiert im Wesentlichen auf Mummendey u.a. (1995: 142-171), der sich für seine Darstellung auf verschiedene empirische Studien sowohl aus dem deutschsprachigen als auch aus dem englischsprachigen, besonders dem amerikanischen Raum beruft. Daher wird hier ebenfalls vorausgesetzt, dass die beschriebenen Techniken, die natürlich allgemein stark kulturabhängig sind, im deutschsprachigen (mitteleuropäischen) und im englischsprachigen (nordamerikanischen) Kulturraum grundsätzlich als vergleichbar betrachtet werden können.

Viele der hier genannten Techniken sind nicht eindeutig zuzuweisen, d. h. dass einige der hier gegebenen Beispiele nicht nur einer, sondern mehreren Kategorien zugeordnet werden könnten. Wie Mummendey u. a. (1995) 137, 139) selber anführt, sind einige der Kategorien eng miteinander verwandt und überlappen sich häufig auch, z. B. ist es häufig unvermeidlich „Offenheit hervorzukehren“, um "Glaub- und Vertrauenswürdigkeit“ zu signalisieren. Und auch das "Widerrufen" und „Rechtfertigen" sind häufig nicht eindeutig voneinander abzugrenzen. Mummendey bezeichnet die von 
ihm zusammengefassten Taxonomien einzelner Impression Management-Techniken entsprechend auch nur als „Ordnungshilfen“ (Mummendey u. a. 1995: 139) und warnt davor, sie als klar umrissen und eindeutig zu betrachten:

Die Aufstellung von Taxonomien des Selbstdarstellungsverhaltens sollte also nicht dazu verführen, jedes mögliche Impression-Management-Verhalten einer bestimmten Kategorie oder Kategorienkombination zweifelsfrei zuordnen zu wollen. (Mummendey u. a.|1995: 139-140)

In Bezug auf das wissenschaftliche Schreiben stellen die kulturellen Konventionen, denen das Verfassen der Textsorte „Wissenschaftlicher Artikel“ unterliegt, bestimmte obligatorische Slots bereit, die dazu dienen, bestimmte Techniken des Impression Managements in bereits weitestgehend ritualisierter Form zu realisieren. Besonders wichtig sind in diesem Zusammenhang sicherlich Danksagungen, die grundsätzlich schon mehrere Zwecke in diesem Sinne erfüllen. Einerseits dienen sie dazu „Glaubwürdigkeit und Vertrauenswürdigkeit herauszustellen“ (credibility, trustworthiness) und „Offenheit hervorzukehren" (self-disclosure), indem sie dem Autor die konventionalisierte Möglichkeit geben, offen zu zeigen, dass er auf die Hilfe anderer zurückgegriffen hat. Dadurch kann man entsprechend auch „Beispielhaft erscheinen“ (exemplification), indem man deutlich macht, welche anderen Personen Einfluss auf den entsprechenden Text hatten und dass man sich nicht "mit fremden Federn schmückt“. Daneben kann der wissenschaftliche Autor hier auch ggf. „Namedropping“ betreiben, um sich über die persönlichen Kontakte zu bekannteren Wissenschaftlern aufzuwerten (BIRGing) und "sich über Kontakte positiv abzuheben“ (boosting). Weiterhin signalisieren sie „Kompetenz und Expertentum" (competence, expertise), insofern, dass sie darauf hindeuten, dass der Autor generell innerhalb einer Expertengruppe etabliert ist ${ }^{32}$ Neben einzelnen und konkreten (Text)Handlungen wie Danksagungen kann darüber hinaus auch die im Rahmen der wissenschaftlichen Ethik notwendige Transparenz, die sich beispielsweise in der Darstellung der Vorgehensweisen, die zu den dargestellten Ergebnissen geführt haben, äußert, als bereits festgeschriebene Form der "Offenheit“ sowie der "Glaub- und Vertrauenswürdigkeit" gedeutet werden.

Im Folgenden werden die hier relevanten Kategorien des Impression Managements nach Mummendey u. a. (1995) kurz vorgestellt und es wird anhand von Beispielen aus dem Korpus erläutert, wie diese Selbstdarstellungstechniken, die vorrangig auf Analysen von Face-to-face-Interaktionen beruhen, auch in wissenschaftlichen Artikeln Anwendung finden können. Dabei ist zu beachten, dass dieser Darstellung keine systematische Analyse des Korpus zugrunde liegt, da dies im Rahmen der vorliegenden

\footnotetext{
${ }^{32}$ Diese Strategien des Impression Management werden natürlich auch von anderen Teiltexten und Handlungen realisiert.
} 
Arbeit zu weit geführt hätte 3 Statt dessen werden hier einige ausgewählte Beispiele für einzelne Techniken gegeben, wobei diese Beispiele gelegentlich auch mehreren Techniken zugeordnet werden können.

\subsubsection{Positives Impression Management}

\section{Eigenwerbung betreiben (self-promotion)}

Eigenwerbung betreiben, d. h. sich selbst positiv darstellen, stellt Mummendey (Mummendey u. a. 1995: 142) als allgemein gebräuchlichste Art des Impression-Managements dar. Dabei ist dies als eine Art Überbegriff zu verstehen, unter dem sich verschiedene Einzeltechniken der Selbstdarstellung zusammenfassen lassen. Ziel aller dieser Techniken ist es, die eigenen Vorzüge herauszustellen und persönliche Fähigkeiten und Leistungen zu betonen. Dabei steht jedoch nicht im Vordergrund, dass man sich bei anderen beliebt machen will, sondern dass man auf andere besonders intelligent, kompetent oder sachverständig wirkt und daher aufgrund der eigenen Kompetenz und Leistungsfähigkeit respektiert wird. Dies kann nach Mummendey sogar dahingehend wirken, dass die betreffende Person sich bei anderen eher unbeliebt macht, allerdings zeige „die tägliche Erfahrung [...], daß eine solch massive Eigenwerbung auf andere Menschen durchaus beeindruckend wirken kann“ (Mummendey u. a.|1995: 142).

Die Relevanz dieser Selbstdarstellungstechniken im wissenschaftlichen Kontext, insbesondere bei der "Vermarktung“ der eigenen Forschung, sei es in wissenschaftlichen Artikeln oder auch Drittmittelanträgen, ist eindeutig nachvollziehbar. Eine wichtige Möglichkeit, in wissenschaftlichen Texten Eigenwerbung zu betreiben, ist sicherlich das Selbstzitat. Hier verweist der Autor auf andere Texte, die er selber geschrieben hat und macht damit deutlich, dass er innerhalb der Community und in dem entsprechenden Themenbereich bereits etabliert ist (womit er weiterreichend auch die Selbstdarstellungstechnik „Expertentum signalisieren“ anwendet). Gerade in einer Wissenschaftskultur in der das Gebot des publish or perish gilt und veröffentlichte Arbeiten als symbolisches Kapital betrachtet werden müssen, das eine Grundvoraussetzung ist für den Erhalt materiellen Kapitals z. B. in Form von bewilligten Forschungsgeldern, ist das Selbstzitat eine der bedeutendsten und angemessensten Formen der Eigenwerbung (Harwood 2005; Hyland|2003, 2001b).

(356) Elsewhere, I discuss how national labor movements' continued orientation toward their nation-states presents significant obstacles to labor transnationalism (Kay 2004b). (Kay 2005)

(357) An anderer Stelle habe ich deren empirische Tragfähigkeit ausführlicher untersucht (vgl. Schulz-Schaeffer 2007). (Schulz-Schaeffer 2009)

\footnotetext{
${ }^{33}$ Aus dem gleichen Grund wird hier auch auf eine weiterreichende kritische Reflexion von Mummendeys Kategorien, insbesondere deren Aufteilung in positives und negatives Impression Management, verzichtet, auch wenn gerade diese Aufteilung sicherlich hinterfragt werden müsste.
} 
Sich über Kontakte aufwerten (BIRGing = basking in reflected glory) und sich über Kontakte positiv abheben (boosting)

Die Technik des BIRGing zeichnet sich dadurch aus, dass man sich mit bestimmten Personen oder (sozialen) Gruppen identifiziert und durch die öffentliche Assoziation mit diesen Personen oder Gruppen deren positive Bewertungen auf sich selber überträgt. Boosting dagegen bedeutet, dass man die Person, mit der man selber zu tun hat und auch öffentlich assoziiert wird, möglichst positiv darstellt und ggf. versucht, ihr öffentliches Image in eine positive Richtung zu modifizieren. Wie oben schon erwähnt ist dies eine der diversen Funktionen, die eine Danksagung in einem wissenschaftlichen Artikel erfüllen kann.

(358) Im internationalen EU-Forschungsprojekt "Socio-economic models of a knowledge-based society" habe ich mit Ulrich Jürgens (Projektleiter) und Martin Krzywdzinski zusammengearbeitet, denen ich hier an erster Stelle meinen Dank ausspreche (zum WZB-Arbeitspaket vgl. Jürgens et al. 2006). (Teipe 2009)

\section{Kompetenz und Expertentum signalisieren (competence, expertise)}

Kompetenz und Fachwissen kann man sicherlich als eine „Währung“ im wissenschaftlichen System verstehen, und die Notwendigkeit, kompetent zu sein und sich fachlich gut zu positionieren ist im Feld „Wissenschaft" eine der wichtigsten Eigenschaften, die ein Akteur haben muss. Für eine Face-to-face-Situation im wissenschaftlichen Kontext gibt Mummendey mit Bezug auf Tedeschi u. a. (1985) folgendes Beispiel:

Wenn man zum Beispiel als junger Wissenschaftler in eine neue Position an einer anderen Universität komme, so könne man nicht verlangen, daß alle dort ansässigen Kollegen über die eigenen wissenschaftlichen Arbeiten, Veröffentlichungen und Projekte Bescheid wüßten. Daher sei es günstig, von Zeit zu Zeit auf die eigenen Qualitäten hinzuweisen - sonst riskiere man es, als jemand angesehen zu werden, der wenig oder nichts tut und so womöglich bei der Verteilung von Mitteln benachteiligt werden könnte. (Mummendey u.a. 1995; 147)

Wie auch die vorangegangenen Kapitel gezeigt haben, ist es ein wesentliches Merkmal einer wissenschaftlichen Identität, kompetent und glaubwürdig zu erscheinen. Daher spielt in wissenschaftlichen Artikeln die Selbstzuweisung von fachlicher Kompetenz eine wichtige Rolle. Als Äquivalent zu obiger Situation könnte hier ebenfalls das Selbstzitat gelten (Beispiel 359). Daneben können auch Hinweise auf andere wichtige wissenschaftliche Erfolge, wie z. B. ein Projektantrag bei einer renommierten Förderinstitution, als Ausdruck dieser Technik interpretiert werden (Beispiel 360).

(359) Elite conflict is the primary threat to elite capacities, yet the interests elites seek to defend are grounded in their relations with the producing classes (I discuss 
this model in more detail elsewhere [Lachmann 2000, chap. 1]). (Lachmann 2009)

(360) Diese begriffliche Präzisierung sowie viele andere relevante Einsichten und Ideen verdanke ich Wolfgang Schluchter, mit dem ich im Frühjahr 2005 ein Forschungsprojekt unter dem Titel „Selig- und Heiligsprechungsverfahren in der katholischen Kirche. Die Repersonalisierung des Charisma im Rahmen seiner Versachlichung" konzipiert und bei der DFG eingereicht habe. (Bienfait 2006)

\section{Beispielhaft erscheinen (exemplification)}

Diese Strategie der Selbstdarstellung bezieht Mummendey vorrangig auf die Demonstration moralischer Integrität. Im wissenschaftlichen Rahmen spielt hier entsprechend die wissenschaftliche Integrität eine Rolle, die sich z. B. darin äußert, dass die Gütekriterien wissenschaftlicher Forschung eingehalten werden etc. Mummendey betont, dass „eine solche Darstellung eigener Beispielhaftigkeit [...] auch als Bemühen gedeutet werden [kann], das Verhalten der anderen zu modifizieren" (Mummendey u.a. 1995 148). Dies unterscheidet diese Strategie von der reinen Eigenwerbung. Mummendey warnt aber auch davor, dass diese Technik schnell ins Gegenteil umschlagen und damit heuchlerisch oder gar lächerlich wirken kann (Mummendey u. a. 1995: 148).

Im Kontext wissenschaftlichen Schreibens kann man diese Technik sicherlich mit der im Rahmen von wissenschaftlicher Redlichkeit notwendigen Transparenz assoziieren. Diese äußert sich nicht zuletzt darin, dass man die eigene Forschungstätigkeit offenlegt und idealerweise auch die eigenen Daten zugänglich macht. Hier ist die enge Verbindung zur Technik „Offenheit hervorkehren“ offensichtlich.

(361) Upon request, I will provide the original series as downloaded. (Gerber 2006)

\section{Hohen Status und Prestige herauskehren (status, prestige)}

Die Begriffe Status und Prestige lassen sich nicht immer eindeutig voneinander trennen. Mummendey versteht hier unter Prestige „in einem bestimmten Sinne “ (Mummendey u. a. 1995 152) eine „nicht unbedingt an eine Rolle, ein Amt, eine Funktion oder Position gebundene Ressource sozialen Einflusses" ; Status fasst er dagegen als „eine durch eine Position legitimierte Autorität". Status und Prestige machen sich insbesondere an sog. Statussymbolen im Sinne des symbolischen Interaktionismus fest. In alltäglichen Face-to-face-Interaktionen äußern sie sich häufig über Kleidung oder materielle Wertgegenstände wie Schmuck, Autos usw. Sie symbolisieren Macht, Wohlstand, Bildung oder andere Werte, die in einer Gesellschaft als besonders oder elitär interpretiert werden (vgl. Mummendey u. a. 1995: 151). Entsprechend kann auch ein akademischer Grad diesen symbolischen Wert darstellen.

In Bezug auf wissenschaftliche Artikel kann entsprechend beispielsweise die Wahl der Zeitschrift, in der der Artikel veröffentlicht wird, einen solchen Wert annehmen. Auch der Verweis auf einen eigenen Artikel, der in einer prestigeträchtigen Zeitschrift 
veröffentlicht wurde, oder auf andere wissenschaftliche Statussymbole (Auszeichnungen, Wissenschaftspreise, Projekte, die von namhaften Institutionen gefördert wurden bzw. werden sollen), könnten in diese Kategorie fallen.

(362) Diese begriffliche Präzisierung sowie viele andere relevante Einsichten und Ideen verdanke ich Wolfgang Schluchter, mit dem ich im Frühjahr 2005 ein Forschungsprojekt unter dem Titel „Selig-und Heiligsprechungsverfahren in der katholischen Kirche. Die Repersonalisierung des Charisma im Rahmen seiner Versachlichung“ konzipiert und bei der DFG eingereicht habe. (Bienfait 2006)

Glaubwürdigkeit und Vertrauenswürdigkeit herausstellen (credibility, trustworthiness)

Ebenso wie die Begriffe Status und Prestige sind auch Glaubwürdigkeit und Vertrauenswürdigkeit nicht ganz einfach voneinander abzugrenzen. Mummendey differenziert dahingehend, dass „,der Begriff der Glaubwürdigkeit [...] sich stärker als derjenige der Vertrauenswürdigkeit am tatsächlichen Zutreffen des von einer glaubwürdigen Quelle vorhergesagten Ereignisses oder Verhaltens zu erweisen" habe (Mummendey u. a. 1995: 152). Diese beiden Eigenschaften ermöglichen es einer Person, der sie zugeschrieben werden oder die es schafft, sich selber als vertrauens- und glaubwürdig zu positionieren, einen deutlichen Einfluss auf die Meinungen und Einstellungen anderer Leute zu nehmen. Beispielsweise wird diese Technik häufig von Lehrern gegenüber ihren Schülern angewandt (Mummendey u. a. 1995: 152). Insofern wäre es nicht verwunderlich, diese Strategie der Selbstdarstellung auch in anderen Kontexten der Wissensvermittlung und -verbreitung wie in wissenschaftlichen Artikeln anzutreffen. "Credibility" wird von Hyland entsprechend auch ein wichtiges Merkmal angesehen, das ein wissenschaftlicher Autor in seinem Text signalisieren muss und wofür er diverse sprachliche Mittel zur Verfügung hat (z. B. Hyland|2002a, 2009, 2010, 2011). Da diese Technik eng verwandt ist mit der Folgenden, Offenheit hervorkehren (self-disclosure) sowie mit Beispielhaft erscheinen (exemplification), sei auf die dortigen Beispiele verwiesen.

\section{Offenheit hervorkehren (self-disclosure)}

Unter "Offenheit hervorkehren" versteht man im allgemeinen eine „vertrauensselige" Person, „die ihr Herz auf der Zunge trägt" (Mummendey u. a. 1995: 153). Dabei ist aber in den meisten Fällen davon auszugehen, dass diesem Verhalten Berechnung zugrunde liegt.

The self-discloser is typically selective, leaking information to others in a manner similar to the way press secretaries work to bolster a politician's public image. (Tedeschi u. a.|1985, 84)

Jemand, der diese Technik anwendet, tut dies, um als beliebter, vertrauenswürdiger, allgemein als sympathischer und attraktiver wahrgenommen zu werden. Damit wird, 
zumindest in der direkten Interaktion, bezweckt, dass sich die Interaktionspartner ebenfalls öffnen und sich dadurch die Möglichkeiten, sie zu beeinflussen, erweitern (Mummendey u. a.|1995: 152).

Im wissenschaftlichen Schreiben ist diese Zielsetzung vermutlich nicht sehr ausgeprägt, allerdings gilt hier sicherlich das Gütekriterium der Transparenz (s. auch Beispielhaft erscheinen (exemplification)), das eine Umsetzung dieser Strategie geradezu einfordert. Wer seinen Forschungsprozess und alles, was dazu gehört, möglichst weit offenlegt, wirkt vertrauenswürdig (und möglicherweise kann das gezielte Einsetzen von Transparenz an der einen Stelle eine gewollte Intransparenz an einer anderen Stelle verbergen).

Weiterhin kann der offene Umgang mit eigenen Fehlern dazu gezählt werden (Beispiel 363). Das Eingestehen von Widersprüchlichkeiten und falschen Schlussfolgerungen in vorhergehenden Arbeiten könnte darüber hinaus auch z. B. Kompetenz und Expertentum signalisieren, da es einen professionellen Umgang mit dem jeweils veränderlichen eigenen Wissen und dem Wissensbestand der Community impliziert (s. auch Widerrufen, ableugnen, dementieren, vorsorglich abschwächen (disclaimers).

(363) In Albert (2005: 395, Fn. 13) habe ich behauptet, dass Lindenbergs bzw. Essers Verfahren der partiellen Definition kein geeignetes Mittel bei der reduktiven Erklärung schwach emergenter Eigenschaften sein kann (vgl. auch Esser 2006: 360). Hier muss ich mich teilweise korrigieren: Für eine bestimmte Kategorie schwach emergenter Eigenschaften scheint mir dies ein gültiges Verfahren reduktiver Erklärung zu sein, insofern keine einfache Zurückführung auf Mikroeigenschaften stattfindet, sondern immer zunächst eine nichttriviale Aggregation stattfindet. (Albert 2005)

(364) Upon request, I will provide the original series as downloaded. (Gerber 2006)

\subsubsection{Negatives Impression Management}

Unter negativen Techniken der Selbstdarstellung versteht Mummendey einerseits Techniken, die nur indirekt darauf ausgerichtet sind, die eigene Person gut darzustellen. Dies wird beispielsweise dadurch erreicht, dass negative Merkmale verneint werden. Daneben gibt es aber auch Techniken, die tatsächlich darauf abzielen, sich selbst in ein negatives Licht zu rücken (Mummendey u. a.|1995: 157-158).

\section{Widerrufen, ableugnen, dementieren, vorsorglich abschwächen (disclaimers)}

Diese Technik wird nach Mummendey u. a. (1995: 162) bereits vorsorglich angewandt, indem der Sprecher oder Schreiber schon vor Eintreten der Situation auf mögliche Schwierigkeiten hinweist, um damit einen schlechten oder ungünstigen Eindruck, der eventuell entstehen könnte, schon im Vorhinein abzuschwächen. 
Diese Technik kommt sicherlich auch häufig im wissenschaftlichen Kontext vor, wo man selbstverständlich davon ausgehen kann, dass andere Mitglieder der Community Unzulänglichkeiten an der eigenen Arbeit suchen und auch finden werden. Der Hinweis auf Aspekte, die aufgrund der Gegebenheiten nicht näher beleuchtet werden können, auf eingeschränkten Zugang zu Daten und darum nicht (so) repräsentative Ergebnisse etc. gehört genauso zum wissenschaftlichen Jargon wie der Verweis auf andere, aus der eigenen Arbeit resultierende Fragestellungen, die jedoch in der jeweils vorliegenden Arbeit nicht berücksichtigt werden können. Dazu zählt auch das verdeutlichen der Forschungsfrage über die negative Darstellung dessen, was man nicht getan hat, um so möglichen Einwänden und Vorwürfen der Community vorzubeugen $\sqrt[34]{34}$

(365) In Albert (2005: 395, Fn. 13) habe ich behauptet, dass Lindenbergs bzw. Essers Verfahren der partiellen Definition kein geeignetes Mittel bei der reduktiven Erklärung schwach emergenter Eigenschaften sein kann (vgl. auch Esser 2006: 360). Hier muss ich mich teilweise korrigieren: Für eine bestimmte Kategorie schwach emergenter Eigenschaften scheint mir dies ein gültiges Verfahren reduktiver Erklärung zu sein, insofern keine einfache Zurückführung auf Mikroeigenschaften stattfindet, sondern immer zunächst eine nichttriviale Aggregation stattfindet. (Albert 2005)

(366) I make no attempt to explain or chronicle emergence and development of the entire "women's movement," going back to its origins in the 1920s (or perhaps earlier). Nor do I deal with major subsequent changes in the movement that took place in the 1970s—especially over the abortion issue —and beyond. (Pedriana 2006)

(367) Lest I be misunderstood, let me clarify what I have not done. First, I have not attempted to turn the sociology of culture on its head. My goal is simply to offer a heuristic model of culture in action that improves upon both the ParsonianWeberian theory of action and the tool kit-repertoire model while preserving the strengths of both. (Vaisey 2009)

\section{Understatement}

Unter Understatement versteht man, wie im alltäglichen Sprachgebrauch, "die mehr oder weniger kontrollierte und taktisch betriebene Untertreibung eigener Fähigkeiten, Leistungen und sonstiger positiver Merkmale“ . Mummendey betrachtet es als „indirekte Technik positiver Eigenwerbung" . Jemand, der Understatement betreibt, geht davon aus, dass sein Gegenüber diese Form der Selbstherabsetzung als nicht ganz ernsthaft und ggf. sogar ironisch erkennt (Mummendey u. a.|1995; 164).

Im wissenschaftlichen Kontext ist diese Form der Selbstdarstellung zwar möglicherweise vorstellbar, aber vielleicht eher in mündlichen Textsorten zu erwarten. Den-

\footnotetext{
${ }^{34}$ Vgl. hierzu auch die umfangreiche Forschung zu Hedging (z. B. Hyland 1998: Graefen 2000).
} 
noch wirkt Mummendeys Bemerkung am Ende seiner Ausführungen zu Understatement nicht deplaziert.

Mit diesen unwesentlichen Bemerkungen soll es hier sein Bewenden haben - leider kennt sich der Verfasser in der Understatement-Forschung nur recht flüchtig aus... (Mummendey u.a.1995, 166)

\section{Abwerten anderer (blasting)}

Das Abwerten anderer bezeichnet Mummendey als negatives Gegenstück zum BIRGing (Mummendey u. a. 1995. 171). Durch die Herabsetzung anderer Personen, Gruppen oder Begebenheiten soll die eigene Person oder, im Fall des wissenschaftlichen Schreibens, sollen die eigenen Aussagen indirekt erhöht werden. In Beispiel 368 wertet der Autor seinen Kollegen, den Soziologen Hartmut Esser, dadurch ab, dass er seine Thesen in Frage stellt und ihm in Beispiel 369, das im Text in der nächsten Zeile folgt, abspricht, seine Ausführungen an anderer Stelle nicht verstanden zu haben. Dadurch kann der Autor seine eigene Konzeption und die von ihm verfolgten Ziele in Abgrenzung zu Esser verdeutlichen und wendet damit zusätzlich die Technik des Rechtfertigens an.

(368) Weiterhin möchte ich zeigen, dass Hartmut Esser in Bezug auf Emergenz bzw. Makrodetermination und Reduktion schwer zu vereinbarende Thesen vertritt. (Albert 2005)

(369) Hartmut Esser (2006: 362) ist es nicht klargeworden, welches Ziel ich mit meiner Konzeption des moderaten methodologischen Holismus, im Konkreten mit dem moderat holistischen Makro-Mikro-Makro-Modell, verfolge: Ersatz, Abgrenzung, Ergänzung oder Aufwertung der historisch-empirischen Perspektive? (Albert 2005)

Wie die obigen Beispiele zeigen, liefert auch die sozialpsychologischer Perspektive lohnende Ausgangspunkte für die Frage nach der Selbstdarstellung und Identitätskonstruktion wissenschaftlicher Autoren. Um hier belastbare Ergebnisse zu erhalten, wäre aber eine deutlich umfassendere Beschäftigung mit den relevanten Theorien und Kategorien notwendig. Insbesondere müssten die hier dargestellten Techniken der Selbstdarstellung stärker differenziert und besser operationalisiert werden.

Ziel dieses Exkurses war es daher nur darzustellen, dass zumindest einige der für direkte Face-to-face-Interaktionen nachgewiesene Selbstdarstellungstechniken auch in wissenschaftlichen Artikeln mit der ersten Person Singular umgesetzt werden und dass wissenschaftliche Autoren regelmäßig auf diese soziale Ressource zurückgreifen, um ihren Einfluss und ihre soziale Macht innerhalb ihrer Community zu erhöhen (Mummendey 2002, 216-217). Dies wurde anhand weniger Beispiele deutlich. Es wäre sicherlich ein Zugewinn, wenn diese Perspektive auf die (sozial)psychologischen 
Grundlagen und Hintergründe wissenschaftlichen Schreibens zukünftig näher beleuchtet würde. 


\section{Kapitel 6}

\section{Didaktische Überlegungen}

Die Auswertung der Selbstdarstellung wissenschaftlicher Autoren anhand der Pronomen der ersten Person im hier untersuchten Korpus hat gezeigt, dass es deutliche Unterschiede gibt, welche Handlungen deutsche und US-amerikanische Autoren auswählen, um sich eine wissenschaftliche Persona zu konstruieren, die in der jeweiligen Wissenschaftskultur als kompetent und damit glaubwürdig wahrgenommen werden kann. Dies ist für sich genommen sicherlich schon eine interessante Information, die aber umso relevanter wird, wenn man über die rein theoretische soziolinguistische Beschreibung dieser Unterschiede hinausgeht und eine anwendungsorientierte Perspektive einnimmt.

Der wichtigste Anwendungsbereich von Ergebnissen aus der Forschung zum wissenschaftlichen Schreiben ist sicherlich die Lehre des wissenschaftlichen Schreibens. Daher sollen die hier erzielten Ergebnisse auf die Möglichkeiten ihrer Anwendung in der Schreibdidaktik hin diskutiert werden. Der Vergleich deutscher und US-amerikanischer Schreibstile legt dabei vor allem den Fokus auf das Schreiben in der jeweilige Fremdsprache nahe, wobei natürlich insbesondere an deutschsprachige Wissenschaftler zu denken ist, die ihre Artikel in englischsprachigen Fachzeitschriften veröffentlichen möchten bzw. sogar müssen. Daneben fällt auch der Nutzen für internationale Studierende sofort ins Auge. Darüber hinaus sind die hier erzielten Ergebnisse aber auch intrakulturell relevant und interessant, wenn es darum geht, Studierende in das wissenschaftliche Schreiben in ihrer L1 einzuführen. Im Folgenden soll der Nutzen dieser Untersuchung für die Lehre des wissenschaftlichen Schreibens kurz dargestellt werden.

Die wissenschaftliche Schreibdidaktik hat in Deutschland im Vergleich zu den englischsprachigen Ländern eine relativ kurze Tradition. Insbesondere die explizite Einbindung in die universitäre Ausbildung im Rahmen von Schreibkursen und Schreibberatungen, häufig angesiedelt in Schreibzentren (sowohl fachspezifisch als auch fachübergreifend), ist eine neuere Erscheinung im deutschen Wissenschaftsbetrieb (Girgensohn 2008). In den meisten Fällen liegt den didaktischen Konzepten heutiger Schreib- 
zentren ein prozessorientierter Ansatz zugrunde (vgl. z. B. Pohl 2007). Was meiner Meinung nach noch nicht ausreichend thematisiert ist, aber ein lohnender zusätzlicher Fokus wäre, ist ein interaktionsorientierter Ansatz der wissenschaftlichen Schreibdidaktik, der neben dem Schreibprodukt und dem Schreibprozess auch die soziale Situiertheit und die kulturelle Bedingtheit des wissenschaftlichen Schreibens explizit berücksichtigt und dabei vor allem die unterschiedlichen Möglichkeiten behandelt, wie Schreibende sich selber in diesem sozio-kulturellen Kontext verorten können. Dass diese Verortung nicht immer nur inhaltlich geschieht, wurde in dieser Arbeit deutlich gezeigt.

Die Selbstdarstellung des Autors, d.h. die Konstruktion einer angemessenen Autorenidentität ist ein wesentlicher Bestandteil erfolgreichen wissenschaftlichen Schreibens. Darüber hinaus ist es gerade für schreibunerfahrene Studierende einer der anspruchsvollsten Aspekte überhaupt, der durch die spezielle Situation vom akademischen Schreiben, d.h. dem Schreiben von quasi-wissenschaftlichen Texten wie z. B. Hausarbeiten zu Trainingszwecken (z. B. Girgensohn und Sennewald 2012, 122)), noch verstärkt wird. Ein grundlegender Aspekt wissenschaftlicher Artikel ist, dass der Autor neues Wissen vermitteln will. Auch wenn dieser Aspekt in dieser Untersuchung nicht im Vordergrund stand, so ist es dennoch ein wichtiges Kriterium wissenschaftlicher Texte, das auch die Möglichkeiten, die ein Autor hat, sich eine angemessene Identität $\mathrm{zu}$ konstruieren, bedeutend mitbestimmt. Studentische Texte unterscheiden sich gerade in diesem Punkt maßgeblich von wissenschaftlichen Texten (Steinhoff 2007: 57), Pohl (2007: 29) spricht entsprechend von einem „im doppelten Sinne Scheinanlass“ (Hervorhebung im Original).

Hinsichtlich der institutionellen Komponente muss es für ihn [den Studierenden, J. S.] darum gehen, sich zu qualifizieren, einen Schein zu erwerben. Hinsichtlich der ideell-fiktiven Komponente hat er den Schein zu erzeugen, als stimmte der Schreibanlass seines Textes mit demjenigen professioneller Texte überein. Ein geteiltes Methoden- und Faktenwissen werden aber ebenso wie gemeinsam geteilte Werte und Normen in diesem Stadium erst noch erworben resp. angebahnt. (Pohl 2007; 29)

Ein weiteres Problem dieser Konstellation, die besonders ungünstig auf die Rollenwahrnehmung der Studierenden und damit auf die Möglichkeit, sich eine eigene Autorenidentität zu konstruieren, rückwirkt, ist das umgekehrte Wissensgefälle zwischen den Studierenden und den Lehrenden, für die sie ihre Hausarbeit verfassen. Der studentische Autor des Textes hat im Normalfall ein weniger umfangreiches (Fach-)Wissen als der Leser bzw. der Dozent. Dies gilt auch und gerade in Bezug auf das Thema des Textes. Dies erzeugt eine paradoxe Situation, denn mit studentischen Texten soll ja gerade auch das Schreiben von wissenschaftlichen Texten, die neue Erkenntnisse vermitteln und das Wissen der Community erweitern, gelernt werden. 
Studierende müssen sich also neben der realen Situation eine fiktive erzeugen, in der sie für eine Community schreiben, deren Wissen sie mit ihrem Text erweitern, oder aber, was vermutlich die häufigere Situation ist, sie schreiben nicht, um spezifisches neues Wissen zu verbreiten, sondern um dem Dozenten $\mathrm{zu}$ demonstrieren, was sie bisher gelernt haben. Dies sind zwei grundlegend verschiedene Ziele, die unterschiedliche Selbstwahrnehmungen begründen und damit auch unterschiedliche Identitäten hervorrufen. Diese Situation („Was soll ich für jemanden schreiben, der das Thema schon hinlänglich und auf jeden Fall besser kennt als ich?“) $]^{1}$ ist für Studierende schwer zu bewältigen und stellt sie immer wieder vor Probleme (vgl. Steinhoff 2007; 58).

Ein weiteres Problemfeld, in dem gerade auch die Selbstdarstellung und damit zusammenhängend die Selbstwahrnehmung und Identitätsbildung von Studierenden eine wesentliche Rolle spielt, ist das Schreiben in einer Fremdsprache. Hier treffen unterschiedliche wissenschaftliche Identitäten und teilweise voneinander abweichende Konzepte von Wissenschaftlichkeit aufeinander. Anders als etablierte Wissenschaftler haben Studierende jedoch auch im Rahmen ihrer ersten wissenschaftskulturellen Sozialisation noch wenig Schreiberfahrung und sind in ihrer eigenen Identität als (junge) Wissenschaftler noch nicht sicher. Daher hat der häufig nicht ausreichend oder sogar gänzlich unbegleitete Wechsel in eine andere Wissenschafts- und Schreibkultur nicht selten zur Folge, dass sie ihre eigene Identität und ihr eigenes Konzept von Wissenschaftlichkeit in Frage stellen müssen. Daraus ergeben sich entsprechend Probleme, ihre eigene Voice zu finden (vgl. z. B. Ivanič und Camps 2001; Kaiser 2002; Eßer 1997). Aber auch für erfahrenere Autoren stellt die Konstruktion einer angemessenen Autorenidentität in der Fremdsprache nicht selten ein Problem dar (Burrough-Boenisch 2003).

Die Frage ist nun, wie diese Probleme in der wissenschaftlichen Schreibdidaktik thematisiert werden können. Daher werden hier einige mehr oder weniger konkrete Vorschläge vorgestellt, wie die sprachliche Selbstdarstellung in wissenschaftlichen Textsorten vermittelt werden kann. In der einschlägigen Ratgeberliteratur wird häufig gegen den Gebrauch der ersten Person geraten und auch das MAN sollte nur vorsichtig eingesetzt werden (z. B. Kornmeier 2008 210). Hierbei wird jedoch übersehen, dass die zugrundelegende Frage nicht ist, ob man es generell darf, sondern welche Mechanismen den Gebrauch steuern und wie man erkennt, an welcher Stelle die erste Person angemessen ist und wo nicht. Da die erste Person, insbesondere im Singular, immer ein Mittel zur sprachlichen Selbstdarstellung des Sprechenden bzw. Schreibenden ist, müssen also den Regeln ihrer Anwendung auch Mechanismen und Regelhaftigkeiten der Selbstdarstellung zugrundeliegen. Die Frage ist also: Wer darf ICH als wissenschaftlicher Autor sein und wie stelle ich diese Persona angemessen dar, d.h. welche

\footnotetext{
${ }^{1}$ Inwiefern diese Einschätzung tatsächlich stimmt und die studentische Arbeit nicht doch Aspekte abdeckt, die dem betreffenden Dozenten nicht oder nicht so gut vertraut sind, sei dahingestellt, die Wahrnehmung, dass man als Student dem Dozenten nichts Neues berichten kann, ist sicherlich der Normalfall.
} 
meiner unzähligen Identitätsfacetten darf ich in meinem Text explizit mir selber zuschreiben, ohne damit meiner Konstruktion einer wissenschaftlichen Persona zu schaden?

Dabei ist zu beachten, dass es hier in keiner Weise darum gehen kann, den Lernenden Vorschriften zu machen und „die richtige“ Art der Selbstdarstellung in wissenschaftlichen Artikeln bzw. Hausarbeiten zu propagieren. Dazu ist dieses Phänomen zu sehr abhängig von den Präferenzen der Fachcommunity und des Autors sowie der Art und des Inhalts des Textes. Es muss vielmehr darum gehen, die Lerner, insbesondere Studierende, die am Anfang ihres akademischen Lernprozesses stehen, zu sensibilisieren und auf die Subtilitäten, die sich in der Konstruktion einer Autorenidentität widerspiegeln, aufmerksam zu machen. Dies könnte anhand von Strategien vermittelt werden, die dann auch auf andere Bereiche übertragen werden können. Dadurch würden die Lernenden in die Lage versetzt, wissenschaftliche Texte und darüber hinaus idealerweise auch andere Texte so zu analysieren, dass sie grundlegenden Textmerkmale erkennen, auch wenn diese nicht vordergründig ins Auge fallen.

In der anglo-amerikanischen Schreibdidaktik ist diese Perspektive vor allem im Rahmen des Voice-Diskurses betrachtet worden (s. z. B. Bowden 1995, 1999; Elbow 1994, 2007; Girgensohn 2008). Hier wird in der Schreibausbildung beispielsweise zwischen schreiber- und leserzentrierten Texten unterschieden (Girgensohn 2008: 206-207), wobei unter schreiberzentrierten Texten diejenigen verstanden werden, die ein Schreibender dazu nutzen kann, sich zunächst selber darüber klar zu werden, über welches Wissen er bereits verfügt und wie er sein Thema innerhalb dieses Wissens einordnen kann. Ein klassisches Verfahren zur Produktion von schreiberzentrierten Texten in diesem Sinne ist das Freewriting (Elbow 1998, 13-19) dessen vorrangiger Sinn es Elbow zufolge ist, dem Schreibenden wieder Zugang zu seiner eigenen Voice zu verschaffen und damit das Schreiben lebendiger zu machen.

If you are a blocked writer, freewriting will help you overcome resistance and move you gradually in the direction of more fluency and control [...]. But if you are a very controlled writer who can write anything you want, but without power-if you have killed the demon-freewriting will gradually bring it back to life. Forcing yourself to write regularly without stopping for ten minutes will put more resistance back into your language. The clay will fight you a bit in your hands as you try to work it into a bowl, but that bowl will end up more alive and powerful. (Elbow 1998; 19)

Eine andere Möglichkeit, Studierende für die unterschiedlichen Möglichkeiten der Selbstdarstellung in wissenschaftlichen Texten, aber auch für andere Aspekte dieser Texte, zu sensibilisieren, schlagen Ken Hyland (Hyland 2002b) und Teresa Thonney (Thonney 2013) vor. Sie plädieren dafür, in der Lehre des wissenschaftlichen Schreiben authentische Korpora mit Texten aus den jeweilige Disziplinen zu verwenden, mit denen 
die Lernenden kleinere empirische Untersuchungen $\mathrm{zu}$ unterschiedlichen textuellen Merkmalen durchführen können. Dies wird auch von Dimitra Koutsantoni befürwortet, die folgende Kriterien für schreibdidaktische Lehrmaterialien aufgestellt hat. Ihrer Meinung nach sollten Lehrmaterialien:

- forschungsbasiert sein

- authentisches Sprachmaterial enthalten

- aus dem jeweiligen disziplinären Kontext stammen und fachliche Inhalte berücksichtigen

- Aufgaben enthalten, die für spezifische sprachliche und kulturelle Besonderheiten sensibilisieren

- und die Studierenden dazu anhalten, selbstständig kleinere Forschungen in dieser Richtung durchzuführen (vgl. Koutsantoni 2007; 221)

Ein übergreifender Aspekt, der die wissenschaftliche Identitätsbildung bei Studierenden stark beeinflusst und erschwert, ist der oben bereits beschriebene Unterschied zwischen akademischen (Trainings-) Texten und wissenschaftlichen Texten (Dittmann u.a. 2003 157). Beim Verfassen akademischer Texte sind die Rollen bedeutend anders verteilt als beim Verfassen wissenschaftlicher Texte. Daher haben Studierende hier eher Schwierigkeiten, eine angemessene wissenschaftliche Identität in ihren Texten zu demonstrieren. Um dieses Problem zu umgehen, ist es sinnvoll, Schreibaufgaben und -kontexte zu schaffen, die dem sozialen Kontext, in dem wissenschaftliche Texte entstehen, möglichst nahe kommen. Dies bedeutet vor allem, dass auch akademische Texte vergleichbar neues Wissen transportieren müssen, so dass die Studierenden nicht mehr nur demonstrieren, was sie gelernt haben, sondern im Rahmen kleinerer Forschungsprojekte neue Erkenntnisse produzieren. Dies würde auch dem Konzept von authentischen Schreibaufgaben in Schreibarrangements entsprechen, wie sie Bräuer und Schindler (2011) vorschlagen:

Als authentisch wird eine Aufgabe in einer Lernumgebung nämlich nur dann von den Akteuren erlebt, wenn sich deren Bemühungen nicht in erster Linie auf eine institutionell definierte Kontrollinstanz (z.B. Fachlehrperson, Lehrmeister in der Berufsausbildung, Vorgesetzte einer Lehrperson) beziehen, sondern auf das im Rahmen einer Schreibaufgabe zu lösende kommunikative Problem. (Bräuer und Schindler 2011: 13)

Dass dies nicht nur im Rahmen der Schreibausbildung wünschenswert ist, sondern auch weiterreichende positive Effekte beispielsweise in der Ausbildung einer fachwissenschaftlichen Methodenkompetenz haben kann, zeigt die aktuelle Entwicklung der Hochschuldidaktik, in der das Konzept des forschungsorientierten Lehrens und Lernens (inquiry-based learning, vgl. Zumbach und Moser (2012)) einen zunehmend grö- 
Beren Raum einnimmt $t^{2}$. Hier werden nicht nur aktuelle Forschungsergebnisse in der Lehre thematisiert, sondern die Lernenden werden selbst als Forschende tätig (Zumbach und Moser|2012 222), d. h. sie produzieren im Rahmen eines kleinen, vom Dozenten angeleiteten Forschungsprojekts neue Erkenntnisse und werten diese anschließend schriftlich aus. Auf diese Weise begeben sie sich in die Rolle des Wissenschaftlers, der diese neuen Erkenntnisse und das neu gewonnene Wissen der Community kommunizieren will und muss. Diese Community könnten im Falle einer forschungsorientierten Lehrveranstaltung beispielsweise die anderen Teilnehmer oder auch andere Kommilitonen im gleichen Studiengang ein, so dass dann eine mögliche Schreibaufgabe in einem solchen Seminar wäre, einen Artikel für diese Seminarcommunity zu schreiben, zu dem die Gruppe anschließend ein Feedback gibt. Durch dieses Peer-Feedback würde, neben vielen anderen Aspekten des wissenschaftlichen Schreibens, auch die Fähigkeit geübt, das Vorwissen der Leser zu antizipieren und den eigenen Text entsprechend adressatenorientiert zu verfassen (Bräuer und Schindler 2011; 15). Dies würde auch den Forderungen von Katrin Girgensohn entsprechen, die insgesamt eine intensivere Auseinandersetzung damit, was wissenschaftliches Schreiben eigentlich ist, für notwendig hält. Ihrer Meinung nach müsste es

den Studierenden deutlicher werden, dass es weniger um Regeln und Gebote geht, als vielmehr darum, Erkenntnis zu produzieren, bzw. produzieren $\mathrm{zu}$ wollen, indem man eine Frage stellt (oder eine These/Hypothese formuliert), die man zu beantworten sucht (oder zu prüfen, belegen), und das methodisch möglichst sauber und unter Bedingungen, die man erläutert. [...] Die Studierenden müssen ein Gefühl für Autorschaft entwickeln, statt sich selbst lediglich als die zu sehen, die Wissen zusammentragen und wiedergeben. Lehrende sollten den Studierenden dabei helfen, eine Selbstwahrnehmung von sich als Autoren zu entfalten. Denn nur wenn das gelingt, erlernen die Studierenden, was Wissenschaft eigentlich ist. (Girgensohn 2008, 208)

Wenn die wissenschaftliche Schreibdidaktik diese Anforderungen erfüllt, wird sich neben vielen anderen Problemen, die Studierende beim Schreiben ihrer Hausarbeiten haben, auch die Frage nach dem ICH-Gebrauch weitestgehend von selber lösen.

\footnotetext{
${ }^{2}$ s. z. B. auch http://www.uni-goettingen.de/de/forschungsorientiertes-lehren-und-lernen/198183. html, letzter Zugriff: 08.08.2013
} 


\section{Kapitel 7}

\section{Zusammenfassung und Ausblick}

Aufgrund der inhärent interaktionalen Situation beim Verfassen wissenschaftlicher Aufsätze, die den sprachlichen und fachkulturellen Anforderungen zur Publikation in einschlägigen Fachzeitschriften genügen sollen, müssen wissenschaftliche Autoren sich selbst als glaubwürdige und kompetente Wissenschaftler in ihren Texten präsentieren. Nur so können sie einerseits die Reviewer und andererseits das breite Fachpublikum von ihren Forschungsergebnissen überzeugen und idealerweise dazu beitragen, dass ihre Artikel zitiert und in den Fachkanon aufgenommen werden. Die Art und Weise, wie diese fachliche Identität sprachlich angemessen konstruiert wird, unterscheidet sich jedoch abhängig von national-, sprach- und fachkulturellen Parametern teilweise erheblich.

In dieser Arbeit wurde die Identitätskonstruktion anhand des Gebrauchs der Pronomen der ersten Person nachgezeichnet. Dazu wurde ein Korpus deutscher und USamerikanischer wissenschaftlicher Texte zusammengestellt, das insgesamt 50 veröffentlichte Texte aus der Kölner Zeitschrift für Soziologie und Sozialpsychologie und dem American Journal of Sociology (insgesam 565851 Wörter) umfasst. Die Texte wurden in der Zeit von 2005 bis 2010 veröffentlicht und aus der Gesamtmenge aller in den beiden Zeitschriften veröffentlichten Artikel, die von einem Einzelautor verfasst wurden, zufällig ausgewählt.

Basierend auf der Annahme, dass wissenschaftliches Schreiben soziale und in den meisten Fällen auch interkulturelle Interaktion ist, wurden zunächst die Grundbegriffe Identität und Rolle definiert und gezeigt, inwiefern Rollen und Identitäten über Handlungen identifiziert werden können. Rollen und Identitäten sowie die Handlungen, die sie symbolisieren, sind immer kulturgebunden und die Zuordnungen von Handlungen zu einer Rolle können sich in unterschiedlichen sozialen und kulturellen Kontexten erheblich voneinander unterscheiden. Aus diesem Grund ist es notwendig, den wissenschaftskulturellen Hintergrund mit zu beachten. Wissenschaftskultur ist abhängig von verschiedenen Faktoren, von denen hier insbesondere der nationale, sprachli- 
che und fachliche Einfluss berücksichtigt wurden. Entsprechend ist auch das Korpus bezüglich dieser Variablen kontrolliert.

Die interaktionale Perspektive auf wissenschaftliches Schreiben ist v. a. in der englischsprachigen Forschung etabliert. Das wichtigste Konzept in diesem Zusammenhang ist Voice, das hier im Rahmen von Ken Hylands Modell von Stance and Engagement berücksichtigt wurde. Der Überblick über den Forschungsstand hat gezeigt, dass die Pronomen der ersten Person, insbesondere im Singular, ein deutliches Mittel sind, mit dem der Autor auf sich selbst referieren kann. Die Verben, mit denen die Pronomen kombiniert werden, geben an, welche Handlungen sich die Autoren über die erste Person selbst zuschreiben. Anhand dieser Handlungen können die sozialen Rollen abgelesen werden, die die Autoren der Identität Wissenschaftlicher Autor zuordnen. Hier ist innerhalb der deutschen bzw. der US-amerikanischen Wissenschaftskultur intrakulturell eine relativ große Übereinstimmung zwischen den jeweiligen Autoren erkennbar, so dass man grundsätzlich allgemein be- und anerkannte Schreibkonventionen annehmen kann. Dennoch sind diese soziokulturellen Konventionen der jeweiligen Diskursgemeinschaft nicht zwingend deterministisch zu betrachten, wie die Fallanalyse des Textes von Diane Vaughan (Vaughan 2006) in Kap. 5.3 zeigt.

Auf der Basis der Ergebnisse aus dem Forschungsüberblick (Kap. 3) wurde ein Kategoriensystem entwickelt, das in der Analyse des Korpus in Kap. 5 angewandt und angepasst wurde. Die beiden Rollen, die in wissenschaftlichen Artikeln (nicht unerwartet) vor allem in der ersten Person umgesetzt werden, sind der Verfasser und der Forscher. Diesen Rollen sind spezifische Handlungen zugeordnet. Der Verfasser handelt dabei textintern als die Person, die den Text strukturiert, auf der Metaebene kommentiert und den Leser führt. Der Forscher dagegen führt im engeren Sinne wissenschaftliche Handlungen aus, die über den Text hinausreichen bzw. nicht direkt mit dem Text in Zusammenhang stehen, insofern, als dass diese Handlungen z. B. auch in anderen Präsentationsformen gleich dargestellt werden könnten. Diese Handlungskategorien umfassen diejenigen Handlungen, die im Lauf des Forschungsprozesses durchgeführt werden, von der Festlegung des Forschungsfokus über die Datenerhebung und -analyse zur Darstellung der Ergebnisse. Weiterhin fällt hierunter auch die Argumentation und das Aufstellen neuer Claims bzw. das Einbringen neuer Ideen und Perspektiven in den wissenschaftlichen Diskurs.

In der Analyse wurden die Texte zunächst in Hinblick auf diese Handlungskategorien untersucht. Die Kategorien, die sich in dieser Untersuchung als relevant herausgestellt haben, wurden in Kapitel 5.1 anhand von Beispielen aus dem Korpus vorgestellt und diskutiert. Der darauf folgende quantitative Vergleich beider Korpora überprüfte die Bedeutung der Einflussfaktoren Sprache, Gender, Forschungsansatz und akademischer Status der Autoren für die untersuchten Pronomen. Wie zu erwarten war, hat der Faktor Sprache bedeutsamen Einfluss auf den Gebrauch der Pronomen in den untersuchten Artikeln. Wie auch in allen vorhergehenden Studien, die das Deutsche und 
das Englische in dieser Hinsicht miteinander verglichen haben (s. Kap. 3.3.1), kamen v. a. die Pronomen der ersten Person Singular in den US-amerikanischen Texten deutlich häufiger vor als in den deutschen. Die anderen Einflussfaktoren wurden daraufhin nur intrakulturell untersucht.

Dabei stellte sich heraus, dass in fast allen Fällen die untersuchten Pronomen häufiger von den männlichen Autoren benutzt wurden. Nur für die erste Person Singular besteht eine leichte Tendenz, dass die amerikanischen Autorinnen sie häufiger benutzten als ihre männlichen Kollegen. Bezüglich des Forschungsansatzes wurde im englischen Korpus eine deutliche Tendenz für die erste Person Singular nachgewiesen, die, entgegen der Ausgangsannahme, von den Autoren quantitativer Texte häufiger benutzt wurde als von den Autoren qualitativer Texte. In den deutschen Texten wurden das WIR und das MAN in den qualitativen Texten häufiger benutzt. Weiterhin wird das ICH von deutschen Professoren häufiger verwendet als von den anderen Statusgruppen im deutschen Teilkorpus, eine Tendenz, die für das Englische nicht nachgewiesen werden konnte. Die englischen Full Professors griffen stattdessen wesentlich häufiger auf das Pluralpronomen zurück als die Vertreter anderer Statusgruppen, eine Tendenz, die auch im Deutschen besteht, wenn auch hier weniger stark ausgeprägt.

Weiterhin hat die Untersuchung ergeben, dass in den englischen Texten ein deutlich größerer Fokus auf Forschungshandlungen liegt, dass also das Forschen im engeren Sinne deutlich stärker in den Vordergrund gerückt wird als in den deutschen Texten. Die eigenen Leistungen der Autoren im Rahmen der Forschung werden stärker betont, beispielsweise machen die Datenerhebung, -aufbereitung und -analyse 40,5\% aller Handlungen aus, die mit der ersten Person Singular im amerikanischen Korpus realisiert wurden (26,76 \% in der ersten Person Plural). Im Deutschen dagegen haben sich nur 7,62 \% der Handlungen, die vom ICH ausgeführt wurden, auf die Datenaufbereitung und -analyse bezogen. Die Handlungen der Datenerhebung schrieben sich die deutschen Autoren gar nicht über das ICH zu. Stattdessen wurden diese Handlungen häufiger mit dem unpersönlichen MAN oder mit anderen unpersönlichen sprachlichen Mitteln (z. B. Passivformen) umgesetzt.

Die Selbstdarstellung als Forscher, insbesondere über das Erheben von und die Arbeit mit den Daten, ist offenbar für die amerikanischen Autoren ein wichtiges Mittel, mit denen sie in ihren Artikeln ihre Glaubwürdigkeit und Kompetenz als Wissenschaftler unter Beweis stellen können. Daher beschreiben sie diese Phase Ihrer Untersuchung auch häufig mit einem deutlichen persönlichen Bezug. Daneben benutzen sie das ICH auch häufig, um zu argumentieren und ihre eigene Position darzustellen, teilweise sogar in explizitem Gegensatz zu anderen Wissenschaftlern. Diese Handlungen sind mehrfach als High-risk-Handlungen beschrieben worden und die Verwendung dieser Handlungen in Kombination mit der ersten Person Singular wurde als ein deutlicher Hinweis auf eine längere Schreiberfahrung und eine „authoritative“ wissenschaftliche Persona gewertet (Hyland 2002a). 
Die deutschen Autoren hingegen schreiben sich hauptsächlich Verfasserhandlungen und weniger Forscherhandlungen zu. Dies ist besonders auffällig bei der Datenerhebung, die die deutschen Autoren gar nicht in Kombination mit dem ICH verwendeten. Und auch die Handlungen der Datenaufbereitung und -analyse schreiben sich nur drei der deutschen Autoren über das ICH selber zu. In den deutschen Texten werden stattdessen hauptsächlich unpersönliche Wendungen bevorzugt und dadurch der Anschein erweckt, dass die Produktion von und die Arbeit mit den Daten in einem unpersönlichen Raum stattfinde. Die Daten „werden erhoben“, von wem ist unklar und offenbar auch unwichtig.

Hier zeigen sich die wissenschaftskulturellen Konventionen, die die verschiedenen möglichen Identitätsslots für die Autoren bereitstellen, sehr deutlich. Durch die unpersönliche und damit (scheinbar) objektive Darstellungsweise dieses zentralen Teils einer (empirischen) Forschung, die die deutschen Autoren bevorzugen, wird die Datenerhebung und Analyse so weit wie möglich von der Person, die sie vollzogen hat, getrennt. Dies ist sicherlich als ein Mittel zu betrachten, mit dem die deutschen soziologischen Autoren möglichen Face-Bedrohungen begegnen, indem sie sich weniger angreifbar machen sowie ihren Daten, Analysen und damit letztlich auch ihren Ergebnissen und Schlussfolgerungen mehr Kraft verleihen. Die US-amerikanischen Autoren demonstrieren ihre Wissenschaftlichkeit demgegenüber gerade darüber, dass sie persönlich die Daten erheben und damit arbeiten. Dadurch wirken die Vorgänge transparenter, die Wissenschaftler reflektieren ihre eigene Subjektivität, demonstrieren ihre methodische Kompetenz und gewinnen so an Glaubwürdigkeit.

Diese beiden Darstellungsformen und die wissenschaftliche Autorenidentität, die über sie kommuniziert wird, sind grundsätzlich gleichwertig und gleichberechtigt und werden in beiden Wissenschaftskulturen als angemessen akzeptiert und verwendet. Dennoch gibt es offenbar Unterschiede in den Konnotationen, die über die Darstellungsform kommuniziert werden. Die möglichen Probleme, die Auftreten können, wenn z. B. ein deutscher Autor seine englischen Texte an den gewohnten deutschen Konventionen ausrichtet, werden deutlich, wenn man bedenkt, dass im Englischen der Gebrauch von Pronomen an der richtigen Stelle mit Kompetenz und Erfahrung assoziiert wird, wohingegen die Vermeidung der ersten Person oder die Verwendung mit überwiegend Low-risk-Handlungen im englischsprachigen Kontext als Zeichen für den studentischen Status und eine noch nicht ausgereifte Schreibkompetenz des Autors gewertet werden kann:

The authorial pronoun is a significant means of promoting a competent scholary identity and gaining acceptance for one's ideas, and while these students were sensitive to its rhetorical effects, they were reluctant to accept its clear connotations of authority and personal commitment. As a result they significantly underused authorial pronouns and determiners, down- 
played their role in the research, and adopted a less clearly independent stance compared with expert writers. (Hyland 2002a: 1110)

Die amerikanischen Autoren stellen sich also offensichtlich sehr viel deutlicher als diejenige Person dar, die „wissenschaftlich“ handelt, in dem Sinne, dass sie Forschungshandlungen durchführen und damit neue wissenschaftliche Erkenntnisse und neues Wissen produzieren. Dabei liegt der Fokus deutlich auf dem individuellen Forscher und nicht auf einer eventuell vorhandenen Forschergruppe. Die deutschen Autoren betonen demgegenüber stärker den gemeinsamen Erkenntnisgewinn und das kollektive Verständnis der Community, indem sie häufig das unpersönliche MAN verwenden und damit die (fiktive) allgemeine Gültigkeit von Schlussfolgerungen und Perspektiven hervorheben.

Dies kann verschiedene Gründe haben. Einerseits wird der US-amerikanischen Kultur generell eine Tendenz zum Individualismus zugeschrieben, die sich auch in verschiedenen Kommunikations- und Interaktionssituationen zeigt (Casper-Hehne 2006). Entsprechend kann man davon ausgehen, dass dieses Prinzip auch in der Wissenschaftskommunikation seinen Niederschlag findet. Dies zeigt sich u.a. daran, dass im US-amerikanischen Kontext die individuelle Forschung einzelner Wissenschaftler einen deutlich höheren Stellenwert besitzt als es in der deutschen Wissenschaft der Fall ist. Während die deutsche Förderpolitik einen stärkeren Fokus auf die Förderung von Gruppenforschung legt, steht in der englischsprachigen, v. a. der US-amerikanischen Wissenschaft die individuelle Förderung von einzelnen Personen im Vordergrund (San. derson|2008a 148). Beispielsweise zeigt der Vergleich von Lebensläufen deutscher und US-amerikanischer Wissenschaftler, dass letztere deutlich mehr Stipendien, Preise und Einzelprojekte enthalten als die ihrer deutschen Kollegen 1 . Weiterhin ist zu bedenken, dass die schreibdidaktische Ausbildung in den USA eine deutlich längere Tradition hat als in Deutschland und dass insbesondere das Konzept Voice, das die individuelle Positionierung der Schreibenden in den Vordergrund stellt, ein wichtiges Leitmotiv in der US-amerikanischen Schreibforschung und -didaktik ist (z. B. Elbow 2007).

Dass die amerikanische (soziologische) Wissenschaftskultur sogar eine überdurchschnittlich ausgeprägte persönliche Darstellungsweise unter bestimmten Umständen akzeptiert, wird in der Fallstudie zum Text von Diane Vaughan (Vaughan 2006) deutlich. Der ICH-Gebrauch in diesem Text weicht sehr stark vom Durchschnitt der anderen Texte ab. Diese Fallstudie zeigt, dass die im Verlauf dieser Arbeit herausgearbeiteten Handlungskategorien und die ihrem Gebrauch zugrundeliegenden Schreibkonventionen nicht deterministisch sind und dass erfahrene Autoren auch eine angemessene wissenschaftliche Identität konstruieren können, wenn sie sich nicht an die

\footnotetext{
${ }^{1}$ Im Vorfeld der Untersuchung wurden die Lebensläufe der Autoren der Korpustexte daraufhin überprüft, in welchem Land und in welchem Fach die Autoren ihre akademische Ausbildung durchlaufen haben, um das Korpus in Hinblick auf die wissenschaftskulturellen Parameter Nation, Sprache und Disziplin zu kontrollieren. In diesem Zusammenhang ergab sich diese Bebachtung
} 
allgemein verfolgten Regeln halten. Dies gilt sogar für extensive autobiographischnarrative Passagen, die im Allgemeinen auch im englischsprachigen Kontext als eindeutig nicht-wissenschaftlich charakterisiert werden. Es liegen der Konstruktion einer glaubwürdigen Autorenpersona also noch andere als die hier untersuchten Einflüsse zugrunde, sehr wahrscheinlich sogar Faktoren, die nicht (nur) linguistisch zu fassen sind.

Eine Perspektive, die einige dieser zusätzlichen Einflussfaktoren berücksichtigen würde, ist die sozialpsychologische, die hier in einem Exkurs angeschnitten wurde. Exemplarisch wurde anhand der Theorie des Impression Management gezeigt, dass die untersuchten wissenschaftlichen Autoren auch die sozialpsychologischen Strategien der Selbstdarstellung verfolgen, die in dieser Theorie beschrieben wurden. Sie betreiben z. B. Eigenwerbung, werten sich über Kontakte mit anderen Wissenschaftlern auf, schwächen ihre Statements vorsorglich ab etc. Diese Strategien führen Sie unter anderem auch anhand der Sätze durch, in denen sie über das Pronomen der ersten Person Singular in ihrem Text auftreten.

Die Ergebnisse dieser Untersuchung sind vor allem für die Schreibdidaktik von besonderem Interesse, die diese interaktionale Perspektive berücksichtigen sollte. Viele Fragen und Unsicherheiten von Studierenden beim Verfassen ihrer akademischen Prüfungstexte wie z. B. Hausarbeiten resultieren aus Unsicherheiten bezüglich ihrer eigenen Position gegenüber ihrer (häufig nur fiktiven) Leserschaft. Indem Studierenden über entsprechende Schreibaufgaben die Möglichkeit gegeben wird, für eine konkrete Adressatengruppe zu schreiben und (für diese Gruppe) in ihren Texten neues Wissen und neue Erkenntnisse zu vermitteln, kann sich eine interaktionale Konstellation ergeben, die es ihnen ermöglicht, sich in ihrer Rolle als studentischer Autor derjenigen des wissenschaftlichen Autors anzunähern. Hierzu ist es notwendig, die sozialen Bedingungen der entsprechenden fachspezifischen Scientific Community mit ihren relevanten Eigenschaften zu emulieren, z. B. im Rahmen von forschungsorientiertem Lernen.

Diese Arbeit hat gezeigt, dass es notwendig ist, auch die interaktionale und damit die soziolinguistische Perspektive auf das wissenschaftliche Schreiben und insbesondere die Selbstdarstellung in wissenschaftlichen Artikeln zu berücksichtigen. Dies kann helfen, diverse weiterreichende Fragen zu beantworten, beispielsweise wie deutsche wissenschaftliche Autoren die oben beschriebenen Höflichkeits- und Impression Management-Strategien auch mit anderen sprachlichen Mitteln einsetzen, ob es deutsche Äquivalente zu Hylands Stance-and-Engagement-Strategien gibt und von welchen weiteren Faktoren eine angemessene wissenschaftliche Identität und ihre Voice im deutschen wissenschaftlichen Schreiben abhängig ist.

Es ist deutlich geworden, dass ein rein linguistischer Ansatz mit einem Fokus auf sprachlichen Funktionen und sprachlichen Handlungen die soziokulturellen und sozialpsychologischen Mechanismen, die der Interaktion und damit der Wissensvermittlung in wissenschaftlichen Artikeln zugrunde liegen, nicht erfassen und daher die- 
ses Phänomen nicht ausreichend ergründen kann. Weitere Untersuchungen müssen entsprechend interdisziplinär vorgehen und z. B. (sozial)psychologische und (wissenschafts)soziologische Perspektiven stärker mit berücksichtigen. Und schließlich sind für derartige Fragestellungen neben deutlich größeren Textkorpora, die andere Fächer sowie andere Textsorten ggf. auch aus diachroner Perspektive enthalten, auch andere Datentypen wie beispielsweise Interviews mit Autoren, Lesern und Peer Reviewern vonnöten. 


\section{Anhang A}

\section{Korpustexte}

\section{A.1 American Journal of Sociology}

[Bartley 2007] BARTLEY, Tim: Institutional Emergence in an Era of Globalization: The Rise of Transnational Private Regulation of Labor and Environmental Conditions. In: American Journal of Sociology 113 (2007), Nr. 2, S. 297-351. - URL http://www. journals.uchicago.edu/doi/abs/10.1086/518871

[Chen 2007] CHEN, Anthony S.: The Party of Lincoln and the Politics of State Fair Employment Practices Legislation in the North, 1945-1964. In: American Journal of Sociology 112 (2007), Nr. 6, S. 1713-1774. - URL http://www.journals.uchicago.edu/ doi/abs/10.1086/512709

[Gerber 2006] GERBER, Theodore P.: Getting Paid: Wage Arrears and Stratification in Russia. In: American Journal of Sociology 111 (2006), Nr. 6, S. 1816-1870. - URL http://www.journals.uchicago.edu/doi/abs/10.1086/499511

[Gibson 2005] GIBSON, David R.: Taking Turns and Talking Ties: Networks and Conversational Interaction. In: American Journal of Sociology 110 (2005), Nr. 6, S. 15611597. - URL http://www.journals.uchicago.edu/doi/abs/10.1086/428689

[Grodsky 2007] GRODSKY, Eric: Compensatory Sponsorship in Higher Education. In: American Journal of Sociology 112 (2007), Nr. 6, S. 1662-1712. - URL http://www. journals.uchicago.edu/doi/abs/10.1086/512707

[Kay 2005] KAY, Tamara: Labor Transnationalism and Global Governance: The Impact of NAFTA on Transnational Labor Relationships in North America. In: American Journal of Sociology 111 (2005), Nr. 3, S. 715-756. - URL http:/ / www.journals.uchicago. edu/doi/abs/10.1086/497305

[King 2008] KING, Ryan D.: Conservatism, Institutionalism, and the Social Control of Intergroup Conflict. In: American Journal of Sociology 113 (2008), Nr. 5, S. 1351-1393. - URL http://www.journals.uchicago.edu/doi/abs/10.1086/525511

[Lachmann 2009] LACHMANN, Richard: Greed and Contingency: State Fiscal Crises and Imperial Failure in Early Modern Europe. In: American Journal of Sociology 115 (2009), Nr. 1, S. 39-73. - URL http://www.journals.uchicago.edu/doi/abs/10.1086/ 597793 
[Loveman 2005] Loveman, Mara: The Modern State and the Primitive Accumulation of Symbolic Power. In: American Journal of Sociology 110 (2005), Nr. 6, S. 1651-1683. - URL http:/ / www.journals.uchicago.edu/doi/abs/10.1086/428688

[Lyons 2007] LYONS, Christopher J.: Community (Dis)Organization and Racially Motivated Crime. In: American Journal of Sociology 113 (2007), Nr. 3, S. 815-863. - URL http://www.journals.uchicago.edu/doi/abs/10.1086/521846

[Madsen 2009] MADSEN, Richard: The Archipelago of Faith: Religious Individualism and Faith Community in America Today. In: American Journal of Sociology 114 (2009), Nr. 5, S. 1263-1301. - URL http://www.journals.uchicago.edu/doi/abs/10. $1086 / 595946$

[Martin 2008] MARTIN, Andrew W.: The Institutional Logic of Union Organizing and the Effectiveness of Social Movement Repertoires. In: American Journal of Sociology 113 (2008), Nr. 4, S. 1067-1103. - URL http:/ / www.journals.uchicago.edu/doi/abs / $10.1086 / 522806$

[Menjívar 2006] MenjÍVAR, Cecilia: Liminal Legality: Salvadoran and Guatemalan Immigrants' Lives in the United States. In: American Journal of Sociology 111 (2006), Nr. 4, S. 999-1037. - URL http://www.journals.uchicago.edu/doi/abs/10. $1086 / 499509$

[Pedriana 2006] PEDRIANA, Nicholas: From Protective to Equal Treatment: Legal Framing Processes and Transformation of the Women's Movement in the 1960s. In: American Journal of Sociology 111 (2006), Nr. 6, S. 1718-1761. - URL http://www. journals.uchicago.edu/doi/abs/10.1086/499911

[Quinn 2008] QUINN, Sarah: The Transformation of Morals in Markets: Death, Benefits, and the Exchange of Life Insurance Policies. In: American Journal of Sociology 114 (2008), Nr. 3, S. 738-780. - URL http://www.journals.uchicago.edu/doi/abs/10. $1086 / 592861$

[Sampson 2008] SAMPSON, Robert J.: Moving to Inequality: Neighborhood Effects and Experiments Meet Social Structure. In: American Journal of Sociology 114 (2008), Nr. 1, S. 189-231. - URL http://www.journals.uchicago.edu/doi/abs/10. $1086 / 589843$

[Schrank 2008] SCHRANK, Andrew: Homeward Bound? Interest, Identity, and Investor Behavior in a Third World Export Platform. In: American Journal of Sociology 114 (2008), Nr. 1, S. 1-34. - URL http://www.journals.uchicago.edu/doi/abs/10.1086/ 588742

[Schwartz 2010] SCHWARTZ, Christine R.: Earnings Inequality and the Changing Association between Spouses' Earnings. In: American Journal of Sociology 115 (2010), Nr. 5, S. 1524-1557. - URL http://www.journals.uchicago.edu/doi/abs/10.1086/ 651373

[Skaggs 2008] SKAGGS, Sheryl: Producing Change or Bagging Opportunity? The Effects of Discrimination Litigation on Women in Supermarket Management. In: American Journal of Sociology 113 (2008), Nr. 4, S. 1148-1182. - URL http:/ / www.journals. uchicago.edu/doi/abs/10.1086/522808 
[Steensland 2006] STEENSLAND, Brian: Cultural Categories and the American Welfare State: The Case of Guaranteed Income Policy. In: American Journal of Sociology 111 (2006), Nr. 5, S. 1273-1326. - URL http://www.journals.uchicago.edu/doi/abs/10. $1086 / 499508$

[Straughn 2005] STRAUGHN, Jeremy B.: Taking the State at Its Word: The Arts of Consentful Contention in the German Democratic Republic. In: American Journal of Sociology 110 (2005), Nr. 6, S. 1598-1650. - URL http:/ / www.journals.uchicago.edu/ doi/abs/10.1086/428818

[Vaisey 2009] VAISEY, Stephen: Motivation and Justification: A Dual-Process Model of Culture in Action. In: American Journal of Sociology 114 (2009), Nr. 6, S. 1675-1715. URL http:/ /www.journals.uchicago.edu/doi/abs/10.1086/597179

[Vallas 2006] VAlLAS, Steven P.: Empowerment Redux: Structure, Agency, and the Remaking of Managerial Authority. In: American Journal of Sociology 111 (2006), Nr. 6, S. 1677-1717. - URL http:/ /www.journals.uchicago.edu/doi/abs/10.1086/499909

[Vaughan 2006] VAughan, Diane: NASA Revisited: Theory, Analogy, and Public Sociology. In: American Journal of Sociology 112 (2006), Nr. 2, S. 353-393. - URL http: //www.journals.uchicago.edu/doi/abs/10.1086/506413

[Viterna 2006] Viterna, Jocelyn S.: Pulled, Pushed, and Persuaded: Explaining Women's Mobilization into the Salvadoran Guerrilla Army. In: American Journal of Sociology 112 (2006), Nr. 1, S. 1-45. - URL http://www.journals.uchicago.edu/doi/ abs/10.1086/502690

\section{A.2 Kölner Zeitschrift für Soziologie und Sozialpsychologie}

[Albert 2005] AlBERT, Gert: Moderater methodologischer Holismus. In: KZfSS Kölner Zeitschrift für Soziologie und Sozialpsychologie 57 (2005), Nr. 3, S. 387-413. - URL http:/ / dx.doi.org/10.1007/s11577-005-0182-9

[Becker 2005] BECKER, Thomas: Liebe: Medium der Kommunikation oder Symbolisches Kapital der Sozialen Reproduktion? In: KZfSS Kölner Zeitschrift für Soziologie und Sozialpsychologie 57 (2005), Nr. 4, S. 624-643. - URL http://dx.doi.org/10.1007/ s11577-005-0218-1

[Berninger 2009] BERNINGER, Ina: Welche familienpolitischen Maßnahmen fördern die Arbeitsmarktpartizipation von Müttern? In: KZfSS Kölner Zeitschrift für Soziologie und Sozialpsychologie 61 (2009), Nr. 3, S. 355-385. - URL http://dx.doi.org/10.1007/ s11577-009-0070-9

[Best 2008] BEST, Henning: Die Umstellung auf ökologische Landwirtschaft. In: KZfSS Kölner Zeitschrift für Soziologie und Sozialpsychologie 60 (2008), Nr. 2, S. 315-339.

- URL http://dx.doi.org/10.1007/s11577-008-0018-5

[Bienfait 2006] BIENFAIT, Agathe: Zeichen und Wunder. In: KZfSS Kölner Zeitschrift für Soziologie und Sozialpsychologie 58 (2006), Nr. 1, S. 1-22. - URL http:/ /dx.doi.org/ 10.1007/s11575-006-0001-1 
[Brose 2008] BROSE, Nicole: Entscheidung unter Unsicherheit - Familiengründung und -erweiterung im Erwerbsverlauf. In: KZfSS Kölner Zeitschrift für Soziologie und Sozialpsychologie 60 (2008), Nr. 1, S. 34-56. - URL http://dx.doi.org/10.1007/ s11577-008-0002-0

[Gerhards 2008] GERHARDS, Jürgen: Die kulturell dominierende Klasse in Europa. In: KZfSS Kölner Zeitschrift für Soziologie und Sozialpsychologie 60 (2008), Nr. 4, S. 723748. - URL http:/ /dx.doi.org/10.1007/s11577-008-0034-5

[Helbig 2010] HELBIG, Marcel: Sind Lehrerinnen für den geringeren Schulerfolg von Jungen verantwortlich? In: KZfSS Kölner Zeitschrift für Soziologie und Sozialpsychologie 62 (2010), Nr. 1, S. 93-111. - URL http:/ / dx.doi.org/10.1007/s11577-010-0095-0

[Isengard 2005] ISENGARD, Bettina: Freizeitverhalten als Ausdruck sozialer Ungleichheiten oder Ergebnis individualisierter Lebensführung? In: KZfSS Kölner Zeitschrift für Soziologie und Sozialpsychologie 57 (2005), Nr. 2, S. 254-277. - URL http: //dx.doi.org/10.1007/s11577-005-0150-4

[Kern 2007] KERN, Thomas: Modernisierung und Demokratisierung. In: KZfSS Kölner Zeitschrift für Soziologie und Sozialpsychologie 59 (2007), Nr. 1, S. 30-58. - URL http://dx.doi.org/10.1007/s11577-007-0002-5

[Lohmann 2010] LOHMANN, Henning: Armut von Erwerbstätigen im europäischen Vergleich. In: KZfSS Kölner Zeitschrift für Soziologie und Sozialpsychologie 62 (2010), Nr. 1, S. 1-30. - URL http:/ /dx.doi.org/10.1007/s11577-010-0093-2

[Lois 2008] LOIS, Daniel: Arbeitsteilung, Berufsorientierung und Partnerschaftsstabilität - Ehen und nichteheliche Lebensgemeinschaften im Vergleich. In: KZfSS Kölner Zeitschrift für Soziologie und Sozialpsychologie 60 (2008), Nr. 1, S. 57-81. - URL http:/ /dx.doi.org/10.1007/s11577-008-0003-z

[Nollmann 2006] NOLLMANN, Gerd: Erhöht Globalisierung die Ungleichheit der Einkommen? In: KZfSS Kölner Zeitschrift für Soziologie und Sozialpsychologie 58 $(2006 / 12 / 01 /)$, Nr. 4, S. 638-659

[Nonnenmacher 2007] NONNENMACHER, Alexandra: Eignen sich Stadtteile für den Nachweis von Kontexteffekten? In: KZfSS Kölner Zeitschrift für Soziologie und Sozialpsychologie 59 (2007), Nr. 3, S. 493-511. - URL http://dx.doi.org/10.1007/ s11577-007-0058-2

[Otte 2005] Отте, Gunnar: Hat die Lebensstilforschung eine Zukunft? In: KZfSS Kölner Zeitschrift für Soziologie und Sozialpsychologie 57 (2005), Nr. 1, S. 1-31. - URL http:/ /dx.doi.org/10.1007/s11577-005-0109-5

[Pollmann-Schult 2006] POLlMANn-SCHult, Matthias: Ausmaß und Struktur von arbeitnehmerinduzierter Abstiegsmobilität. In: KZfSS Kölner Zeitschrift für Soziologie und Sozialpsychologie 58 (2006), Nr. 4, S. 573-591. - URL http://dx.doi.org/10.1007/ s11577-006-0257-2

[Rössel 2006/09/23/] RÖSSEL, Jörg: Kostenstruktur und Ästhetisierung? In: KZfSS Kölner Zeitschrift für Soziologie und Sozialpsychologie 58 (2006/09/23/), Nr. 3, S. 453-467 
[Schnabel 2005] SCHNABEL, Annette: Gefühlvolle Entscheidung und entscheidende Gefühle. In: KZfSS Kölner Zeitschrift für Soziologie und Sozialpsychologie 57 (2005), Nr. 2, S. 278-307. - URL http:/ / dx.doi.org/10.1007/s11577-005-0151-3

[Schulz-Schaeffer 2009] SCHULZ-SCHAEFFER, Ingo: Handlungszuschreibung und Situationsdefinition. In: KZfSS Kölner Zeitschrift für Soziologie und Sozialpsychologie 61 (2009), Nr. 2, S. 159-182. - URL http:/ /dx.doi.org/10.1007/s11577-009-0049-6

[Schwinn 2006] SCHWINN, Thomas: Konvergenz, Divergenz oder Hybridisierung? In: KZfSS Kölner Zeitschrift für Soziologie und Sozialpsychologie 58 (2006), Nr. 2, S. 201232. - URL http:/ /dx.doi.org/10.1007/s11575-006-0053-2

[Stauder 2008] STAUDER, Johannes: Opportunitäten und Restriktionen des Kennenlernens. In: KZfSS Kölner Zeitschrift für Soziologie und Sozialpsychologie 60 (2008), Nr. 2, S. 266-286. - URL http:/ / dx.doi.org/10.1007/s11577-008-0016-7

[Strauß 2009] STRAUSS, Susanne: Ehrenamt in Deutschland und Großbritannien Sprungbrett zurück auf den Arbeitsmarkt? In: KZfSS Kölner Zeitschrift für Soziologie und Sozialpsychologie 61 (2009), Nr. 4, S. 647-670. - URL http://dx.doi.org/10.1007/ s11577-009-0085-2

[Strein 2005] STREIN, Petra: Soziale Mobilität und Lebensstile. In: KZfSS Kölner Zeitschrift für Soziologie und Sozialpsychologie 57 (2005), Nr. 2, S. 205-229. - URL http: //dx.doi.org/10.1007/s11577-005-0148-y

[Teipen 2008] TEIPEN, Christina: Hochtechnologische Unternehmen im Spiegel des „Varieties-of-Capitalism“-Ansatzes. In: KZfSS Kölner Zeitschrift für Soziologie und Sozialpsychologie 60 (2008), Nr. 4, S. 765-787. - URL http://dx.doi.org/10.1007/ s11577-008-0036-3

[Trappe 2006] TRAPPE, Heike: Berufliche Segregation im Kontext. In: KZfSS Kölner Zeitschrift für Soziologie und Sozialpsychologie 58 (2006), Nr. 1, S. 50-78. - URL http: //dx.doi.org/10.1007/s11575-006-0003-z 


\section{Literaturverzeichnis}

[Abels 2006] ABELS, Heinz: Identität: Über die Entstehung des Gedankens, dass der Mensch ein Individuum ist, den nicht leicht zu verwirklichenden Anspruch auf Individualität und die Tatsache, dass Identität in Zeiten der Individualisierung von der Hand in den Mund lebt. 1. Aufl. Wiesbaden : VS Verlag für Sozialwissenschaften, 2006

[Abels 2009] ABELS, Heinz: Einführung in die Soziologie. 4. Aufl. Wiesbaden : VS Verlag für Sozialwissenschaften, 2009

[Adamzik 2002] ADAMZIK, Kirsten: Interaktionsrollen. Die Textwelt und ihre Akteure. In: ADAMZIK, Kirsten (Hrsg.): Texte - Diskurse - Interaktionsrollen. Tübingen : Stauffenburg-Verl., 2002, S. 211-255 (Textsorten, 6)

[Ahrenholz 2008] AHRENHOLZ, Bernt: Erstsprache - Zweitsprache-Fremdsprache. In: AHRENHOLZ, Bernt (Hrsg.) ; OOMEN-WELKE, Ingelore (Hrsg.): Deutsch als Fremdsprache. Baltmannsweiler : Schneider Hohengehren, 2008, S. 3-16 (Deutschunterricht in Theorie und Praxis, 9)

[Aksnes 2003] AKSNES, Dag W.: A macro study of self-citation. In: Scientometrics 56 (2003), Nr. 2, S. 235-246

[Androutsopoulos 2001] ANDROUTSOPOULOS, Jannis: Von fett zu fabelhaft: Jugendsprache in der Sprachbiografie. In: CÖLFEN, Hermann (Hrsg.): Hypermedien und Wissenskonstruktion. Oldenburg : Red. OBST, 2001, S. 55-78 (Osnabrücker Beiträge zur Sprachtheorie, 63)

[Auer und Baßler 2007a] AuER, Peter (Hrsg.) ; BASSLER, Harald (Hrsg.): Reden und Schreiben in der Wissenschaft. Frankfurt am Main : Campus, 2007

[Auer und Baßler 2007b] Auer, Peter ; BASSleR, Harald: Der Stil der Wissenschaft. In: AUER, Peter (Hrsg.) ; BASSLER, Harald (Hrsg.): Reden und Schreiben in der Wissenschaft. Frankfurt am Main : Campus, 2007, S. 9-29

[Bazerman 1988] BAZERMAN, Charles: Shaping written knowledge: The genre and activity of the experimental article in science. Madison and Wis. : Univ. of Wisconsin Pr., 1988 (Rhetoric of the human sciences)

[Bazerman u. a. 2005] BAZERMAN, Charles ; LitTle, Joseph ; BETHEL, Lisa ; CHAVKIN, Teri ; FOUQUETTE, Danielle ; GARUFIS, Janet: Reference guide to writing across the curriculum. West Lafayette Ind. : Parlor Press and WAC Clearinghouse, 2005 (Reference guides to rhetoric and composition)

[Benninghaus 2007] BENNINGHAUs, Hans: Deskriptive Statistik: Eine Einführung für Sozialwissenschaftler. 11. Aufl. Wiesbaden : VS Verlag für Sozialwissenschaften, 2007 
[Benwell und Stokoe 2006] BENWELL, Bethan ; STOKOE, Elizabeth: Discourse and identity. Edinburgh : Edinburgh Univ. Press, 2006

[Bourdieu 1998] BOURDIEU, Pierre: Vom Gebrauch der Wissenschaft: Für eine klinische Soziologie des wissenschaftlichen Feldes. Konstanz : UVK Univ.-Verl., 1998 (UVK Soziologie, 12)

[Bowden 1995] BowDEN, Darsie: The Rise of a Metaphor: "Voice" in Composition Pedagogy. In: Rhetoric Review 14 (1995), Nr. 1, S. 173-188

[Bowden 1999] BowDEN, Darsie: The Mythology of voice. Portsmouth and NH : Boynton/Cook, 1999

[Bräuer und Schindler 2011] BRÄUER, Gerd ; SCHINDLER, Kirsten: Schreibarrangements für Schule, Hochschule, Beruf. Freiburg : Fillibach, 2011

[Brown und Levinson 1987] BROWN, Penelope ; Levinson, Stephen C.: Politeness: Some universals in language usage. Cambridge / New York : Cambridge University Press, 1987

[Bucholtz und Hall 2005] BuCHOLTZ, Mary ; HALL, Kira: Identity and interaction: a sociocultural linguistic approach. In: Discourse Studies 7 (2005), Nr. 4-5, S. 585-614

[Bucholtz und Hall 2008] BuchOlTz, Mary ; HALL, Kira: Finding identity: Theory and data. In: Multilingua - Journal of Cross-Cultural and Interlanguage Communication 27 (2008), Nr. 1-2, S. 151-163

[Burrough-Boenisch 2003] BURROUGH-BOENISCH, Joy: Shapers of published NNS research articles. In: Journal of Second Language Writing 12 (2003), Nr. 3, S. 223-243

[Burrough-Boenisch 2005] BURROUGH-BOENISCH, Joy: NS and NNS scientists' amendments of Dutch scientific English and their impact on hedging. In: English for Specific Purposes 24 (2005), Nr. 1, S. 25-39

[Casper-Hehne 2006] CASPER-HEHnE, Hiltraud: Deutsch-amerikanische Alltagskommunikation: Zur Beziehungsarbeit in interkulturellen Gesprächen. Tübingen : Niemeyer, 2006

[Cherry 1988] CHERRY, Roger D.: Ethos Versus Persona: Self-Representation in Written Discourse. In: Written Communication 5 (1988), Nr. 3, S. 251-276

[Clyne 1991] CLYNE, Michael: Zu kulturellen Unterschieden in der Produktion und Wahrnehmung englischer und deutscher wissenschaftlicher Texte. In: Info DaF 18 (1991), Nr. 4, S. 376-383

[Dittmann u.a. 2003] DitTMAnN, Jürgen ; Geneuss, Katrin A. ; Nennstiel, Christoph ; QUAST, Nora A.: Schreibproblerne im Studium - Eine empirische Untersuchung. In: EHLICH, Konrad (Hrsg.): Wissenschaftlich schreiben - lehren und lernen. Berlin [u.a.] : de Gruyter, 2003, S. 155-185

[Ehlich 2007] EHLICH, Konrad: Sprache und sprachliches Handeln: Band 3: Diskurs Narration - Text - Schrift. Berlin [u.a.] : de Gruyter, 2007

[Elbow 1994] ELBOW, Peter (Hrsg.): Landmark essays on voice and writing. Davis and CA : Hermagoras Press, 1994 
[Elbow 1998] ELBOW, Peter: Writing with power: Techniques for mastering the writing process. 2. Aufl. New York : Oxford University Press, 1998

[Elbow 2007] ElBOW, Peter: Voice in Writing Again: Embracing Contraries. In: College English 70 (2007), Nr. 2, S. 168-188

[Emcke 2000] EMCKE, Carolin: Kollektive Identitäten: Sozialphilosophische Grundlagen. Frankfurt [u.a.] : Campus-Verl, 2000

[Eßer 1997] ESSER, Ruth: Etwas ist mir geheim geblieben am deutschen Referat: Kulturelle Geprägtheit wissenschaftlicher Textproduktion und ihre Konsequenzen für den universitären Unterricht von Deutsch als Fremdsprache. München : Iudicium Verlag, 1997

[Fandrych und Graefen 2002] FANDRYCH, Christian ; GRAEFEN, Gabriele: Text commenting devices in German and English academic articles. In: Multilingua 21 (2002), S. $17-43$

[Fløttum u.a. 2006] Fløttum, Kjersti ; GedDE-DAhl, Trine ; KINN, Torodd: Academic voices: Across languages and disciplines. Amsterdam : Benjamins, 2006 (Pragmatics \& Beyond New Series, 148)

[Flowerdew 1999] FLOWERDEW, John: Problems in writing for scholarly publication in English: The case of Hong Kong. In: Journal of Second Language Writing 8 (1999), Nr. 3, S. 243-264

[Foster 2006] FOSTER, David: Writing with authority: Students' roles as writers in crossnational perspective. Carbondale : Southern Illinois University Press, 2006

[Galtung 1985] GALTUNG, Johan: Struktur, Kultur und intellektueller Stil. Ein vergleichender Essay über sachsonische, teutonische, gallische und nipponische Wissenschaft. In: WIERLACHER, Alois (Hrsg.): Das Fremde und das Eigene. München : Iudicium Verlag, 1985, S. 151-192

[Gerhardt 1971] GERHARDT, Uta: Rollenanalyse als kritische Soziologie: Ein konzeptioneller Rahmen zur empirischen und methodologischen Begründung einer Theorie der Vergesellschaftung. Neuwied : Luchterhand, 1971 (Soziologische Texte, 72)

[Gerhardt 2008] GerhARDT, Uta: Rolle / Role. In: TRUdGILL, Peter (Hrsg.) ; MATTHEIER, Klaus J. (Hrsg.) ; DITTMAR, Norbert (Hrsg.): Sociolinguistics / Soziolinguistik (Part 1). 2. Aufl. Mouton de Gruyter, 2008, S. 384-391 (Handbücher zur Sprachund Kommunikationswissenschaft / Handbooks of Linguistics and Communication Science / [HSK], 3/1)

[Giannoni 2002] GIANNONI, Davide S.: Worlds of Gratitude: A Contrastive Study of Acknowledgement Texts in English and Italian Research Articles. In: Applied Linguistics 23 (2002), Nr. 1, S. 1-31

[Girgensohn 2008] GIRGENSOHN, Katrin: Schreiben, als spreche man nicht selbst. Über die Schwierigkeiten von Studierenden, sich in Bezug zu ihren Schreibaufgaben zu setzen. In: RotHe, Matthias (Hrsg.): Stil, Stilbruch, Tabu. Berlin and Münster : Lit, 2008, S. 195-211

[Girgensohn und Sennewald 2012] GIRGEnsohn, Katrin ; SEnNEwALD, Nadja: Schreiben lehren, Schreiben lernen: Eine Einführung. Darmstadt: WBG, 2012 
[Goffman 1961] GOFFMAN, E.: Encounters: two studies in the sociology of interaction. Bobbs-Merrill, 1961 (The advanced studies in sociology series)

[Goffman 1959] GOFFMAN, Erving: The presentation of self in everyday life. Garden City and NY (u.a.) : Doubleday, 1959 (Anchor books, 174)

[Goffman 1971] GofFMAN, Erving: Relations in public; Microstudies of the public order. New York : Basic Books, 1971

[Goffman 1973] GOFFMAN, Erving: Interaktion: Spass am Spiel, Rollendistanz. München : R. Piper, 1973 (Serie Piper, 62)

[Gotti 2009a] GotTI, Maurizio (Hrsg.): Commonality and individuality in academic discourse. Bern : Peter Lang Verlag, 2009 (Linguistic insights, 100)

[Gotti 2009b] GotTI, Maurizio: Introduction. In: GotTI, Maurizio (Hrsg.): Commonality and individuality in academic discourse. Bern : Peter Lang Verlag, 2009, S. 9-21

[Graefen 1994] GRAEFEN, Gabriele: Wissenschaftstexte im Vergleich: Deutsche Autoren auf Abwegen? In: BRÜNNER, Gisela (Hrsg.) ; GRÄFEN, Gabriele (Hrsg.): Texte und Diskurse. Opladen : Westdt. Verl., 1994, S. 136-157

[Graefen 1997] GRAEFEN, Gabriele: Der wissenschaftliche Artikel: Textart und Textorganisation. Frankfurt am Main and Berlin : Lang, 1997 (Arbeiten zur Sprachanalyse, 27)

[Graefen 2000] GRAEFEN, Gabriele: "Hedging" als neue Kategorie? Ein Beitrag zur Diskussion. - URL http://www.daf.uni-muenchen.de/media/downloads/hedge.pdf (Zugriff: 08.08.2016)

[Graefen 2007] GRAEFEN, Gabriele: Wissenschaftliches Schreiben: Von der Hausarbeit zur Magisterarbeit. - URL http://www.daf.uni-muenchen.de/media/downloads/ anleitung_haus.pdf (Zugriff: 08.08.2016)

[Graefen und Thielmann 2007] GRAEFEN, Gabriele ; THIELMANN, Winfried: Der wissenschaftliche Artikel. In: AuER, Peter (Hrsg.) ; BASSLER, Harald (Hrsg.): Reden und Schreiben in der Wissenschaft. Frankfurt am Main : Campus, 2007, S. 67-97

[Graffin u.a. 2011] GrafFin, Scott D. ; CARPENTER, Mason A. ; BoIVIE, Steven: What's all that (strategic) noise? Anticipatory impression management in CEO succession. In: Strategic Management Journal 32 (2011), Nr. 7, S. 748-770

[Halliday 1994] Halliday, M. A. K.: An introduction to functional grammar. 2. Aufl. London : Arnold, 1994

[Harwood 2005] HARWOOD, Nigel: 'Nowhere has anyone attempted ... In this article I aim to do just that': A corpus-based study of self-promotional I and we in academic writing across four disciplines. In: Journal of Pragmatics 37 (2005), Nr. 8, S. $1207-1231$

[Hermanns 2012] HERMANNS, Fritz: Sprache, Kultur und Identität. In: KäMPER, Heidrun (Hrsg.) ; LinKE, Angelika (Hrsg.) ; Wengeler, Martin (Hrsg.) ; HeRMANNS, Fritz (Hrsg.): Der Sitz der Sprache im Leben. Berlin : de Gruyter, 2012 
[Hinkel 1999] HINKEL, Eli (Hrsg.): Culture in second language teaching and learning. Cambridge / New York : Cambridge University Press, 1999 (Cambridge Applied Linguistics Series)

[Hirvela und Belcher 2001] HiRvelA, Alan ; BELCHER, Diane: Coming back to voice: The multiple voices and identities of mature multilingual writers. In: Journal of Second Language Writing 10 (2001), Nr. 1-2, S. 83-106

[Hufeisen 2002] HUFEISEN, Britta: Ein deutsches Referat ist kein englischsprachiges Essay: Theoretische und praktische Überlegungen zu einem verbesserten textsortenbezogenen Schreibunterricht in der Fremdsprache Deutsch an der Universität. Innsbruck : Studien Verlag, 2002 (Theorie und Praxis : Österreichische Beiträge zu Deutsch als Fremdsprache, 5)

[Hutz 1997] Hutz, Matthias: Kontrastive Fachtextlinguistik für den fachbezogenen Fremdsprachenunterricht. Fachzeitschriftenartikel der Psychologie im interlingualen Vergleich. Wissenschaftlicher Verlag, Trier, 1997 (SALS - Studien zur anglistischen Literatur- und Sprachwissenschaft, 8)

[Hyland 1998] HylAnD, Ken: Hedging in Scientific Research Articles. Amsterdam/Philadelphia : John Benjamins Publishing Company, 1998 (Pragmatics and beyond, 54)

[Hyland 2000] HylAND, Ken: Disciplinary Discourses: Social Interactions in Academic Writing. Harlow : Pearson Education Ltd., 2000 (Applied linguistics and language study)

[Hyland 2001a] HYLAND, Ken: Bringing in the Reader. In: Written Communication 18 (2001), Nr. 4, S. 549-574

[Hyland 2001b] HYLAND, Ken: Humble Servants of the Discipline? Self-Mention in Research Articles. In: English for Specific Purposes 20 (2001), S. 2007-2226

[Hyland 2002a] HYLAND, Ken: Authority and invisibility: authorial identity in academic writing. In: Journal of Pragmatics 34 (2002), S. 1091-1112

[Hyland 2002b] HYLAND, Ken: Options of identity in academic writing. In: ELT Journal 56 (2002), Nr. 4, S. 351-358

[Hyland 2003] HYLAND, Ken: Self-citation and self-reference: Credibility and promotion in academic publication. In: Journal of the American Society for Information Science and Technology 54 (2003), Nr. 3, S. 251-259

[Hyland 2004] HYLAND, Ken: Disciplinary differences: language variation in academic discourses. In: HYLAND, Ken (Hrsg.) ; BONDI, Marina (Hrsg.): Academic discourse across disciplines. Bern : Peter Lang Verlag, 2004, S. 17-43

[Hyland 2005] HYLAND, Ken: Stance and engagement. A model of interaction in academic discourse. In: Discourse Studies 7 (2005), Nr. 2, S. 173-192

[Hyland 2008] HYLAND, Ken: Disciplinary voices: Interactions in research writing. In: English Text Construction 1 (2008), Nr. 1, S. 5-22 
[Hyland 2009] HYLAND, Ken: Constraint vs Creativity: Identity and Disciplinarity in Academic Writing. In: GotTI, Maurizio (Hrsg.): Commonality and individuality in academic discourse. Bern : Peter Lang Verlag, 2009, S. 25-52

[Hyland 2010] HYLAND, Ken: Community and Individuality: Performing Identity in Applied Linguistics. In: Written Communication 27 (2010), Nr. 2, S. 159-188

[Hyland 2011] HYLAND, Ken: Disciplines and Discourses: Social Interactions in the Construction of Knowledge. In: STARKe-Meyerring, Doreen (Hrsg.) ; PARÉ, Anthony (Hrsg.) ; ARTemevA, Natasha (Hrsg.) ; Horne, Miriam (Hrsg.) ; YOUSOUBOVA, Larissa (Hrsg.): Writing in Knowledge Societies. Fort Collins and Colorado : The WAC Clearinghouse and Parlor Press, 2011, S. 193-214. - URL http: //wac.colostate.edu/books/winks/chapter10.pdf (Zugriff: 08.08.2016)

[Hyland und Bondi 2004] Hyland, Ken (Hrsg.) ; BONDI, Marina (Hrsg.): Academic discourse across disciplines. Bern : Peter Lang Verlag, 2004. (Linguistic insights, 42)

[Ivanič 1998] IVANIČ, Roz: Writing and identity. The discoursal construction of identity in academic writing. Amsterdam/Philadelphia : John Benjamins Publishing Company, 1998 (Studies in written language and literacy, 5)

[Ivanič und Camps 2001] IVANIČ, Roz ; CAMPS, David: I am how I sound: Voice as self-representation in L2 writing. In: Voice in L2 Writing 10 (2001), Nr. 1-2, S. 3-33

[Jackson und Lilleker 2011] JACKSON, Nigel ; LILLEKER, Darren: Microblogging, Constituency Service and Impression Management: UK MPs and the Use of Twitter. In: The Journal of Legislative Studies 17 (2011), Nr. 1, S. 86-105

[Jakobs 1997] JAKOBS, Eva-Maria: Textproduktion als domänen- und kulturspezifisches Handeln: Diskutiert am Beispiel wissenschaftlichen Schreibens. In: ADAMZIK, Kirsten (Hrsg.) ; ANTOS, Gerd (Hrsg.) ; JAKOBS, Eva-Maria (Hrsg.): Domänen- und kulturspezifisches Schreiben. Frankfurt am Main and New York : Peter Lang Verlag, 1997

[Johansson 2002] JOHANSSON, Stig: Viewing languages through multilingual corpora, with special reference to the generic person in English, German, and Norwegian. In: Languages in Contrast 4 (2002), Nr. 2, S. 261-280

[Junge 2009] JUNGE, Matthias: Kultursoziologie: eine Einführung in die Theorien. Konstanz : UVK-Verl.-Ges., 2009 (UTB, 3299)

[Kaiser 2002] KAISER, Dorothee: Wege zum wissenschaftlichen Schreiben: Eine kontrastive Untersuchung zu studentischen Texten aus Venezuela und Deutschland. Tübingen : Stauffenburg Verlag, 2002 (Textsorten, 5)

[Kaplan und Baldauf Jr. 2005] KAPLAN, Robert B. ; BALDAUF JR., Richard B.: Editing contributed scholarly articles from a language management perspective. In: Journal of Second Language Writing 14 (2005), Nr. 1, S. 47-62

[Kornmeier 2008] KORNMEIER, Martin: Wissenschaftlich schreiben leicht gemacht: Für Bachelor, Master und Dissertation. 1. Aufl. Bern [u.a.] : Haupt, 2008 (UTB, 3154) 
[Kostouli 2005a] Kostoul, Triantafillia: Introduction: Making Social Meanings in Contexts - Writing, Literate Activities and Learning Processes in Sociohistorical Communities. In: Kostoul, Triantafillia (Hrsg.): Writing in Context(s): Textual Practices and Learning Processes in Sociocultural Settings. New York : Springer Science+Business Media Inc., 2005

[Kostouli 2005b] Kostouli, Triantafillia (Hrsg.): Writing in Context(s): Textual Practices and Learning Processes in Sociocultural Settings. New York : Springer Science+Business Media Inc., 2005 (Studies in Writing, 15)

[Koutsantoni 2007] KOUTSANTONI, Dimitra: Developing academic literacies: Understanding disciplinary communities' culture and rhetoric. Bern/Oxford : Peter Lang Verlag, 2007 (Contemporary studies in descriptive linguistics, 4)

[Kresic 2006] KRESIC, Marijana: Sprache, Sprechen und Identität: Studien zur sprachlichmedialen Konstruktion des Selbst. München : Iudicium Verlag, 2006

[Kresta 1995] KRESTA, Ronald: Realisierungsformen der Interpersonalität in vier linguistischen Fachtextsorten des Deutschen und des Englischen. Frankfurt/M. : Peter Lang Verlag, 1995 (Theorie und Vermittlung der Sprache, 24)

[Kretzenbacher und Weinrich 1995] KRETZENBACHER, Heinz L. (Hrsg.) ; WeINRICH, Harald (Hrsg.): Linguistik der Wissenschaftssprache. Berlin and New York: Walter de Gruyter, 1995 (Forschungsbericht, 10)

[Kuo 1999] KUO, Chih-Hua: The Use of Personal Pronouns: Role Relationships in Scientific Journal Articles. In: English for Specific Purposes 18 (1999), Nr. 2, S. 121-138

[Kussmaul 1978] Kussmaul, Paul: Kommunikationskonventionen in Textsorten, am Beispiel deutscher und englischer geisteswissenschaftlicher Abhandlungen: Ein Beitrag zur deutsch-englischen Übersetzungstechnik. In: Lebende Sprachen 23 (1978), Nr. 2, S. 54-60

[Le Page und Tabouret-Keller 1985] LE PAGE, Robert B. ; TABOURET-KelleR, Andrée: Acts of identity: Creole-based approaches to language and ethnicity. Cambridge u.a : Cambridge Univ. Pr., 1985

[Leech 1991] LEECH, Geoffrey: The state of the art in corpus linguistics. In: AIJMER, Karin (Hrsg.) ; AltenberG, Bengt (Hrsg.) ; SvARTVIK, Jan (Hrsg.): English corpus linguistics. Harlow : Longman, 1991, S. 8-29

[Llamas und Watt 2010] LlamAS, Carmen (Hrsg.) ; WATT, Dominic James L. (Hrsg.): Language and identities. Edinburgh : Edinburgh University Press, 2010

[Lucius-Hoene und Depperman 2004] Lucius-Hoene, Gabriele ; DePPERMAN, Arnulf: Narrative Identität und Positionierung. In: Gesprächsforschung - OnlineZeitschrift zur verbalen Interaktion (2004), Nr. 5, S. 166-183. - URL http://www. gespraechsforschung-ozs.de/heft2004/ga-lucius.pdf (Zugriff: 08.08.2016)

[Mair 2008] MAIR, Christian: Cult of the informal? On some ongoing changes in scientific English. In: GNUTZMANN, Claus (Hrsg.): English in academia. Tübingen : Narr, 2008, S. 93-106 
[McEnery u. a. 2006] MCENERY, Tony ; TONO, Yukio ; XIAO, Richard: Corpus-based language studies: An advanced resource book. London [u.a.] : Routledge, 2006

[Merkl-Davies und Brennan 2011] Merkl-DaviES, Doris M. ; BrennAN, Niamh M.: A conceptual framework of impression management: new insights from psychology, sociology and critical perspectives. In: Accounting and Business Research 41 (2011), Nr. 5, S. 415-437

[Merkl-Davies u.a. 2011] Merkl-Davies, Doris M. ; Brennan, Niamh M. ; MCLEAY, Stuart J.: Impression management and retrospective sense-making in corporate narratives: A social psychology perspective. In: Accounting, Auditing $\mathcal{E}$ Accountability Journal 24 (2011), Nr. 3, S. 315-344

[Moebius 2009] MoEBIUS, Stephan: Kultur. Bielefeld : transcript, 2009 (Einsichten)

[Mühlhäusler und Harré 1990] MÜHLHÄUSLER, Peter ; HARRÉ, Rom: Pronouns and people: The linguistic construction of social and personal identity. Oxford u. a. : B. Blackwell, 1990

[Mummendey 2002] MuMmendey, Hans D.: Selbstdarstellungstheorie. In: FREY, Dieter (Hrsg.) ; IRLE, Martin (Hrsg.): Motivations-, Selbst- und Informationsverarbeitungstheorien. Bern : H. Huber, 2002, S. 212-233

[Mummendey u. a. 1995] Mummendey, Hans D. ; EIfler, Stefanie ; MELCHER, Werner: Psychologie der Selbstdarstellung. 2. Aufl. Göttingen: Hofgrefe-Verlag für Psychologie, 1995

[Myers 1989] MYERS, Greg: The pragmatics of politeness in scientific articles. In: Applied Linguistics 10 (1989), Nr. 1, S. 1-35

[Pogner 1997] PogNer, Karl-Heinz: Diskursgemeinschaft und Interaktion: Zum Schreiben von beratenden IngenieurInnen. In: ADAMZIK, Kirsten (Hrsg.) ; ANTOS, Gerd (Hrsg.) ; JAKOBS, Eva-Maria (Hrsg.): Domänen- und kulturspezifisches Schreiben Frankfurt am Main and New York: P. Lang, 1997, S. 127-150. (Textproduktion und Medien, 3) - URL http:/ / www.prowitec.rwth-aachen.de/p-publikationen/ band-pdf/band3/band3_pogner.pdf (Zugriff: 08.08.2016)

[Pogner 1999] POGNER, Karl-Heinz: Textproduktion in Diskursgemeinschaften. In: JAKOBS, Eva-Maria (Hrsg.) ; KNORR, Dagmar (Hrsg.) ; POGNER, Karl-Heinz (Hrsg.): Textproduktion Frankfurt am Main and New York : P. Lang, 1999, S. 145158. (Textproduktion und Medien, 5) - URL http://www.prowitec.rwth-aachen. de/p-publikationen/band-pdf/band5/band05_pogner.pdf (Zugriff: 08.08.2016)

[Pogner 2003] POGNER, Karl-Heinz: Writing and interacting in the discourse community of engineering: Pragmatics of Writing. In: Journal of Pragmatics 35 (2003), Nr. 6, S. 855-867

[Pohl 2007] POHL, Thorsten: Studien zur Ontogenese wissenschaftlichen Schreibens. Tübingen : Niemeyer, 2007 (Reihe Germanistische Linguistik, 271)

[Rayson und Garside 2000] RAYSON, Paul ; GARSIDE, Roger: Comparing corpora using frequency profiling. In: KILGARRIFF, Adam (Hrsg.) ; BERBER SARDINHA, Tony (Hrsg.): Proceedings of the workshop on Comparing corpora - Volume 9. Association for 
Computational Linguistics (ACL) : New Brunswick, 2000, S. 1-6. - URL http:// eprints.lancs.ac.uk/11882/1/rg_acl2000.pdf (Zugriff: 08.08.2016)

[Reckwitz 2008] RECKWITZ, Andreas: Subjekt. Bielefeld : transcript, 2008 (Einsichten)

[Rosenberg und Egbert 2011] RosenberG, Jenny ; EGBERT, Nichole: Online Impression Management: Personality Traits and Concerns for Secondary Goals as Predictors of Self-Presentation Tactics on Facebook. In: Journal of Computer-Mediated Communication 17 (2011), Nr. 1, S. 1-18. - URL http://dx.doi.org/10.1111/j.1083-6101. 2011.01560.x (Zugriff: 08.08.2016)

[Sanderson 2005] SANDERSON, Tamsin: Kontrastive Fachtextpragmatik deutschund englischsprachiger wissenschaftlicher Texte: Danksagungen im interlingualen Vergleich. In: ADAMZIK, Kirsten (Hrsg.) ; KRAUSE, Wolf-Dieter (Hrsg.): Text-Arbeiten. Tübingen : G. Narr, 2005, S. 59-83

[Sanderson 2008a] SANDERSON, Tamsin: Corpus, culture, discourse Tübingen : G. Narr, 2008 (Language in performance, 39)

[Sanderson 2008b] SANDERSON, Tamsin: Interaction, identity and culture in academic writing. In: ÄDEL, Annelie (Hrsg.) ; REPPEN, Randi (Hrsg.): Corpora and discourse Amsterdam : Benjamins, 2008, S. 57-92 (Studies in Corpus Linguistics, 31)

[Selting und Couper-Kuhlen 2000] SELTING, Margret ; COUPER-KuHLEN, Elisabeth: Argumente für die Entwicklung einer 'interaktionalen Linguistik'. In: Gesprächsforschung - Online-Zeitschrift zur verbalen Interaktion 1 (2000), S. 76-95. - URL http:// www.gespraechsforschung-online.de/heft2000/ga-selting.pdf (Zugriff: 08.08.2016)

[Steinhoff 2007] STEINHOFF, Torsten: Wissenschaftliche Textkompetenz: Sprachgebrauch und Schreibentwicklung in wissenschaftlichen Texten von Studenten und Experten Tübingen : Niemeyer, 2007 (Reihe Germanistische Linguistik, 280)

[Swales 1990] SWALES, John M.: Genre analysis. English in academic and research settings. Cambridge University Press, Cambridge, 1990 (Cambridge Applied Linguistics Series)

[Tang und John 1999] TANG, Ramona ; JOHN, Suganthi: The [']I' in identity: Exploring writer identity in student academic writing through the first person pronoun. In: English for Specific Purposes 18 (1999), Nr. Supplement 1, S. S23 - S39

[Tannen 1985] TANNEN, Deborah: Frames and Schemas in Interaction. In: RASKIN, Victor (Hrsg.): Quaderni di Semantica's Round Table Discussion on Frame/Script Semantics Bd. 6. Società editrice il Mulino, 1985, S. 326-335.

[Tardy und Matsuda 2009] TARDY, Christine M. ; MATSUdA, Paul K.: The Construction of Author Voice by Editorial Board Members. In: Written Communication 26 (2009), Nr. 1, S. 32-52

[Tedeschi u.a. 1985] Tedeschi, James T. ; LindSKOLD, Svenn ; Rosenfeld, Paul: Introduction to social psychology. St. Paul and Minn : West Pub. Co., 1985

[Thielmann 2009] THIELMANN, Winfried: Deutsche und englische Wissenschaftssprache im Vergleich: Hinführen - Verknüpfen - Benennen. Heidelberg : Synchron Wiss.-Verl. der Autoren, 2009 (Wissenschaftskommunikation, 3) 
[Thomas 1991] ThOMAS, Alexander: Grundriß der Sozialpsychologie: Band 1: Grundlegende Begriffe und Prozesse. 1. Aufl. Göttingen [u.a.] : Hogrefe, 1991

[Thonney 2013] THONNEY, Teresa: "At first I thought... but I don't know for sure": The Use of First Person Pronouns in the Academic Writing of Novices. In: Across the Disciplines 10 (2013), Nr. 1. - URL http:/ / wac.colostate.edu/atd/articles / thonney2013.cfm (Zugriff: 08.08.2016)

[Trumpp 1998] TRUMPP, Eva C.: Fachtextsorten kontrastiv: Englisch-deutsch-französisch. Tübingen : Gunter Narr, 1998

[Vassileva 2000] VASSILEVA, Irina: Who is the author? A contrastive analysis of authorial presence in English, German, French, Russian and Bulgarian academic discourse. Sankt Augustin : Asgard Verlag, 2000

[Vaughan 1996] VAUGHAN, Diane: The Challenger launch decision: Risky technology, culture, and deviance at NASA. Chicago : University of Chicago Press, 1996

[Vester 2009] VeSTER, Heinz-Günter: Kompendium der Soziologie I: Grundbegriffe. 1. Aufl. Wiesbaden : VS Verlag für Sozialwissenschaften, 2009

[Vester 2010] VESTER, Heinz-Günter: Kompendium der Soziologie III: Neuere soziologische Theorien. 1. Wiesbaden : VS Verlag für Sozialwissenschaften, 2010

[Weingart 2003] WEINGART, Peter: Wissenschaftssoziologie. Bielefeld : Transcript-Verl., 2003 (Einsichten)

[Weinrich 1989] WEINRICH, Harald: Formen der Wissenschaftssprache. In: Jahrbuch 1988 der Akademie der Wissenschaften zu Berlin (1989), S. 119-158

[Zhao und Llosa 2008] ZHAO, Cecilia G. ; LlOSA, Lorena: Voice in high-stakes L1 academic writing assessment: Implications for L2 writing instruction. In: Assessing Writing 13 (2008), Nr. 3, S. 153-170

[Zumbach und Moser 2012] ZuMBACH, Jörg ; MOSER, Stephanie: Problembasierte Lehre als Methode zur Umsetzung forschungsorientierter Hochschullehre in der LehrerInnenausbildung. In: BREZOWAR, Gabriela (Hrsg.) ; MAIR, Michael (Hrsg.) ; OlsOWSKI, Gunter (Hrsg.) ; ZuMBACH, Jörg (Hrsg.): Problem-Based Learning im Dialog. Wien : Facultas, 2012, S. 221-232. - URL https://www.sbg.ac.at/mediaresearch/ zumbach/download/2011_2012/Zumbach_Moser_12b.pdf (Zugriff: 08.08.2016) 